\title{
100 Areas CERCLA Ecological Investigations
}

Prepared for the U.S. Department of Energy Environmental Restoration and Waste Management

\footnotetext{
Qe Westinghouse

(2.) Hanford Company Richland, Washington

Hanford Operations and Engineering Contractor for the

U.S Dupartment of Energy under Contract DE AC06 87RL.10930
} 


\section{LEGAL DISCLAIMER}

This report was prepared as an account of work sponsored by an agency of the United States Government. Neither the United Stales Government nor any agency thereot, nor any of their employees, nor any of their contractors, subcontractors or their employees, makes any warraniy, express or implied, or assumes any legal liability or responsibility for the accuracy, completeness, or any third party's use or the results of such us $\theta$ of any information, apparatus, product, or process disclosed, or represeri's that its use would not infringe privalely owned rights. Reference herein to any specilic commercial product, process, or service by trade name, trademark, manufacturer, or otherwise, does not necessarily constilute or imply its endorsement, recommendation, or favoring by the Unifod States Government or any agency thereof or its contractors or subcontractors. The viows and opinions of authors expressed herein do nol necessarily state or reflect those of the United States Government or any agency thereot.

This report has been reproduced from the best available copy Available in paper copy and microliche.

Available to the U.S. Department of Energy and its contractors from

Office of Scientific and Technical Information P.O. Box 62

Oak Ridge. TN 37831

(615) $576-8401$

Available to the public from the U.S. Department of Commerce National Technical Information Service

5285 Port Royai tinad

Springfield, VA 22161

(703) 487.4650

Ptinted in the United States ol America

DISCLM.) CHP (1.91) 


\section{Areas CERCLA Ecological Investigations}

D. S. Landeen

M. R. Sackschewsky

S. Weiss

Date Published

September 1993

Prepared for the U.S. Department of Energy Environmental Restoration and Waste Management

(2) Westingthouse P.O. Box 1970

Hanford Company Richland, Washington 99352

Hantord Operations and Engineering Contractor for the

U.S. Department of Energy under Contract DE-AC06-87RL.10930 
WOWMAL

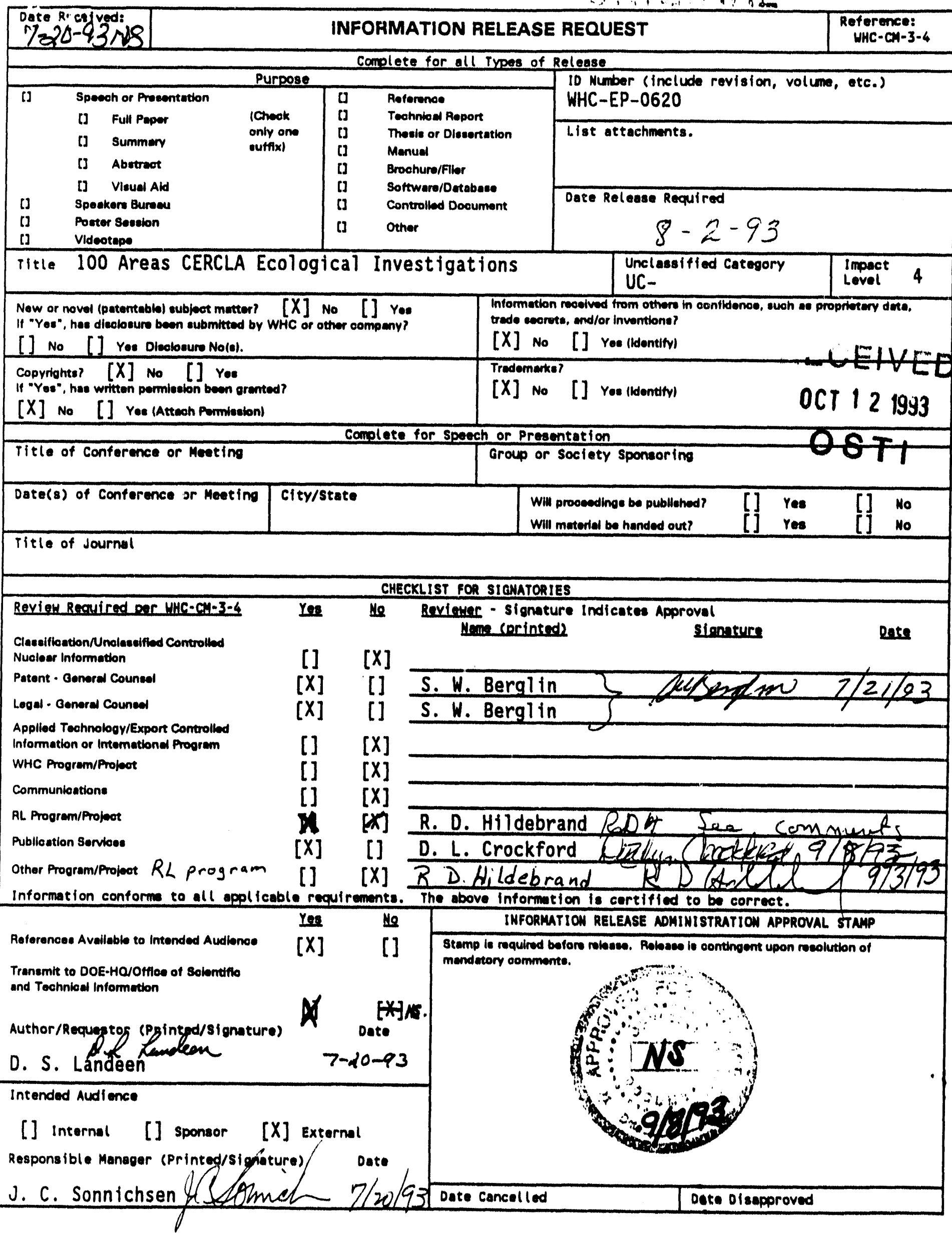


WHC-EP-0620

\section{EXECUTIVE SUMMARY}

This document reports the results of the field terrestrial ecological investigations conducted by Westinghouse Hanford Company during fiscal years 1991 and 1992 at operable units 100-FR-3, 100-HR-3, 100-NR-2, 100-KR-4, and 100-BC-5. The tasks reported here are part of the Remedial Investigations conducted in support of the Comprehensive Environmental Response, Compensation, and Liability Act of 1980 studies for the 100 Areas. These ecological investigations provide (1) a description of the flora and fauna associated with the 100 Areas operable units, emphasizing potential pathways for contaminants and species that have been given special status under existing state and/or federal laws, and (2) an evaluation of existing concentrations of heavy metals and radionuclides in biota associated with the 100 Areas operable units.

The 1991 and 1992 Westinghouse Hanford Company field investigations concentrated on the following: bird surveys, mammal and insect surveys, vegetation surveys, and biota sampling which included asparagus, reed canarygrass, trees, milfoil, raptor (birds of prey) pellets, coyote scat, and soil from ant mounds and small mammal burrow excavations.

Evidence of burrowing by small mammals and/or harvester ants was documented. Soil samples collected from small mammal burrows and ant mounds associated with these sites showed very low or undetectable levels of contaminants.

Concentrations of metals and radionuclides in coyote scat and raptor pellets were generally very low. Average lead concentrations in both coyotes and raptors were higher near the 100 Areas operable units than in samples 
WHC -EP-0620

collected in control areas. Further studies would be needed to determine if these lead concentrations are correlated to roadside traffic, which has been documented elsewhere in the United States.

Vegetation samples (asparagus, reed canarygrass, tree leaves and limbs, and milfoil) were collected at several locations upriver and downriver of the operable units of interest. Results from these sampling efforts indicate little or no biological uptake of radionuclides or inorganic waste constituents, with the most notable exception of elevated strontium-90 concentrations (up to $88 \mathrm{pCi} / \mathrm{g}$ ) in mulberry trees near the 100-BC and $100-K$ reactors.

The data presented in this report represent a substantial amount of information that can be used for comparative purposes in future sampling efforts at the Hanford Site. Also, this report provides details concerning the 100 Areas terrestrial ecology that can be used to support future remedial actions and clean-up measures.

The information presented in this report also includes previously published information contained in Landeen and Sackschewsky (1992), Fiscal Year 1991100 Areas CERCLA Ecological Investigations. 


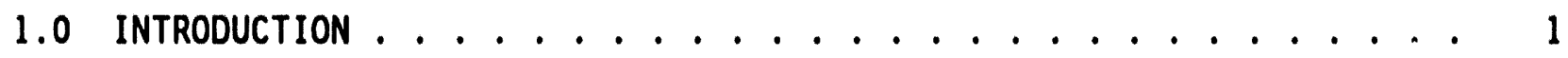

2.0 SCOPE OF WORK . . . . . . . . . . . . . . . 2

2.1 OBJECTIVES $\ldots . \ldots 2$

2.2 FIELD INVESTIGATIONS ................. 3

3.0 BIRD SURVEYS . . . . . . . . . . . . . . . 3

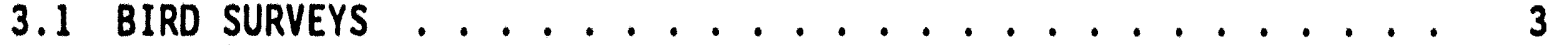

3.2 POTENTIALLY SIGNIFICANT BIRD SPECIES . . . . . . . . . 4

4.0 INSECT AND MAMMAL SURVEYS . . . . . . . . . . . . . . 17

4.1 MAMMALS . . . 17

4.2 MAMMAL AND INSECT SURVEYS AT INDIVIDUAL WASTE SITES . . . . 17

5.0 WILDLIFE SURVEY CONCLUSIONS . . . . . . . . . . . 18

6.0 PLANT COMMUNITY ANALYSES . . . . . . . . . . . . . . 23

6.1 PLANT COMMUNITY DELINEATIONS ............... 23

6.1 .1 100-BC Area ................. 24

$6.1 .2100-k$ Area . . . . . . . . . . . . . . . 25

$6.1 .3100-\mathrm{N}$ Area . . . . . . . . . . . . . . . 25

$6.1 .4100-0$ Area . . . . . . . . . . . 26

6.1.5 Riparian Communities in the 100-HR-3 Operable Unit. . 26

$6.1 .6100-$ H Area . . . . . . . . . . . . . . . 27

$6.1 .7100-$ F Area . . . . . . . . . . . 28

6.2 THREATENED AND ENDANGERED PLANT SPECIES . . . . . . . . . 28

6.3 OTHER SPECIES OF INTEREST . . . ............ 31

7.0 BIOTA SAMPLING . . . . . . . . . . . . . . 33

7.1 ASPARAGUS ............................ 33

7.2 REED CANARYGRASS .................. 33

7.3 TREE LEAVES AND LIMBS . . . . . . . . . . . . . . . . . . 33

7.4 WATER MILFOIL . . . . . . . . . . . . . . . . 33

7.5 ANIMALS (COYOTES AND RAPTORS) . . . . . . . . . 34

7.6 ANT MOUNDS AND SMALL MAMMAL BURROWS ............. 34

8.0 DATA ANALYSIS . . . . . . . . . . . . . 34

8.1 SOURCES OF METALS AND RADIONUCLIDES ............ 44

8.2 ASPARAGUS $\ldots \ldots \ldots$. . . . . . . . . . 47

8.3 REED CANARYGRASS .................... 49

8.4 TREE LEAVES AND LIMBS . . . . . . . . . . . . . . . 49

8.5 WATER MILFOIL $\ldots \ldots$. . . . . . . . 50

8.6 ANIMALS (COYOTES AND RAPTORS) $\ldots \ldots \ldots$

8.7 ANT MOUNDS $\ldots \ldots \ldots \ldots$

8.8 SMALL MAMMAL BURROWS ................ 56

9.0 SUMMARY AND RECOMMENDATIONS . . . . . . . . . . . . . . 57

9.1 DATA EVALUATION PROBLEMS . . . . . . . . . . . . . . 57

9.2 SUMMARY

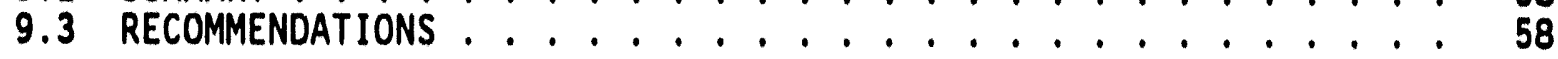


CONTENTS (cont.)

10.0 REFERENCES ...................... 59

11.0 BIBLIOGRAPHY .................... 67 APPENDIXES

A SAMPLE LOCATION MAPS FOR ALL MEDIA .............. A-1

B ANALYTICAL RESULTS FOR ASPARAGUS AND MILFOIL ........... B-1

C ANALYTICAL RESULTS FOR REED CANARYGRASS ............ . c-1

D ANALYTICAL RESULTS FOR TREES ................ D . . .

E ANALYTICAL RESULTS FOR COYOTES AND RAPTORS ........... E-1

F ANALYTICAL RESULTS FOR ANT MOUNDS .............. F-1

G ANALYTICAL RESULTS FOR SMALL MAMMAL BURROWS ........... G G-1

H RADIONUCLIDE VALUES IN SOIL AND VEGETATION FROM 100 AREAS COLLECTED AS PART OF WESTINGHOUSE HANFORD COMPANY'S ENVIRONMENTAL MONITORING PROGRAM .............. H-1

I PLANT SPECIES OBSERVED AT 100 AREAS OPERABLE UNITS . . . . . . . . I-1

J SELECTED METALS CONCENTRATIONS IN ALL MEdiA . . . . . . . . . J-1

K MARION OWNBEY HERBARIUM LETTERS ................ K-1 
WHC-EP-0620

\section{LIST OF FIGURES}

1 Zinc Concentration in Asparagus (1992) ........... 48

2 Strontium-90 Concentrations at Reactor Sites (1992) . . . . . . 51

3 Copper and Zinc Concentrations at Reactor Sites (1991 and 1992) . . 52

4 Antimony, Cadmium, Lead, and Zinc Concentrations at N Springs (1992) .................... 53

$5 \quad$ Zinc and Lead Concentrations for Coyote Scat and Raptor Pellets (1992) ................... 55

A-1 Sample Locations and Identification Numbers for Asparagus

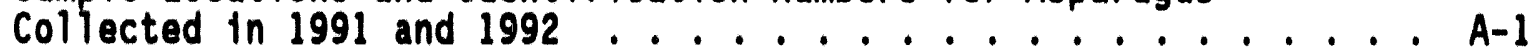

A-2 Sample Locations and Identification Numbers for Reed Canarygrass Collected in 1991 and 1992 . . . . . . . . . . A-2

A-3 Sample Locations and Identification Numbers for Tree and

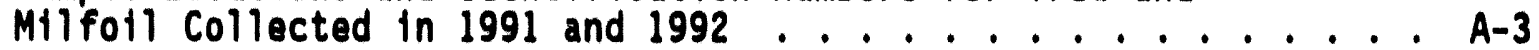

A-4 Sample Locations and Identification Numbers for Raptor Pellet and Coyote Scat Collected in 1992 .............. A-4

A-5 Sample Locations and Identification Numbers for Ant Mounds

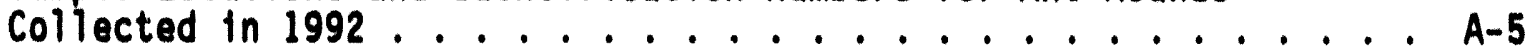

A-6 Sample Locations and Identification Numbers for Small Mammal

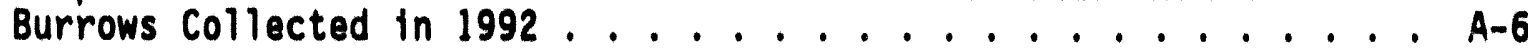

I-1 100 Areas Operable Units ................... I-1 
WHC-EP-0620

\section{LIST OF TABLES}

1 Federal and State Status Codes for Special Plants and Animals... 5

2 Federal and State Classifications of Wildlife and Plant Spectes at the Hanford Site.................. 7

3 Birds Observed at 100 Areas Operable Units . . . . . . . . . 13

4 List of All the Mammals Observed and Known To Inhabit the 100 Areas Operable Units .................. 19

5 Insect and Mammal Surveys at 100 Areas Operable Units . . . . . . 20

6 Hanford Site Endangered, Threatened, and Sensitive

Plant Spectes ..................... 29

7 Hanford Site Edible Plants ............... 32

8 Ant Mound and Burrow Sampling Locattons .............. 35

9 Average Values of all Control Samples Collected in 1991 and 1992 as Part of 100 Areas Ecological Investigations and other Studies. .................. 36

10 Average Values of all Samples Collected in 1991 and 1992 as Part of 100 Areas Ecological Investigations and other studtes ........................ 38

11 Average Values for Sediments Collected in the 100 Areas ...... 40

12 Soll Background Values (ppm) for Selected Metals Reported for the Hanford Site ... . . . . . . . . . . . . . . . . . 41

13 Soll Threshold Values (ppm) and Cleanup Standards ......... 42

14 Maxima and 95/95 Reference Thresholds for Sitewide Soll Background ... . . . . . . . . . . . . . . . . . . 43

15 Toxicity Levels of Selected Metals in Plants ........... 45

B-1 Asparagus Sample Results Collected from 100 Areas in 1991 . . . . B-1

B-2 Asparagus Sample Results Collected from 100 Areas in $1992 \ldots \ldots$

B-3 Milfoll Results Collected at N Springs in $1992 \ldots \ldots$. . . . . . . B-4

C-1 Reed Canarygrass Sample Results from 100-F Reactor Area

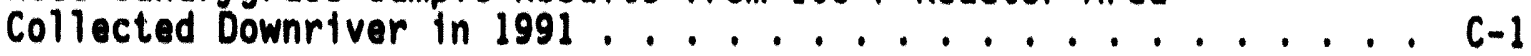

C-2 Reed Canarygrass Sample Results from 100-H Reactor Area Collected Upriver in $1991 \ldots \ldots$. . . . . . . . . . C C-2 


\section{LIST OF TABLES (cont.)}

C-3 Reed Canarygrass Sample Results from 100-H Reactor Area

Collected Downrtver in 1991.................... C..3

C-4 Reed Canarygrass Sample Results from 100-0 Reactor Area

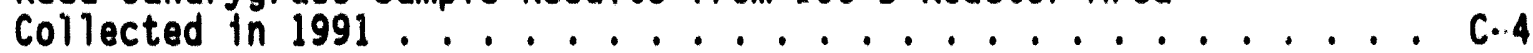

C-5 Reed Canarygrass Duplicate Sample Results from 100-D Reactor

Area Collected in 1991 .................. C-5

C-6 Reed Canarygrass Sample Results Collected Below 100-D Reactor

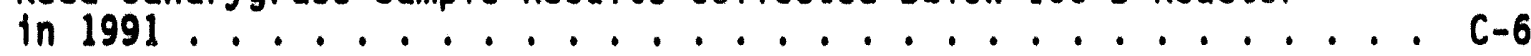

C-7 Reed Canarygrass Sample Results irom 100-BC Reactor Area

Collected Downrtver in 1991 . . . . . . . . . . . . . . C-7

C-8 Reed Canarygrass Control Sample Results from 1991 . . . . . . . . . C-8

C-9 Reed Canarygrass Sample Results from 100-F Renctor Area

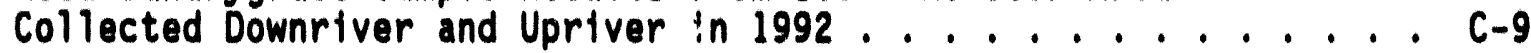

C-10 Reed Canarygrass Sample Results from 100-H Reactor Area

Collected Downriver and Upriver in 1992............. C-10

C-11 Reed Canarygrass Sample Results from 100-D Reactor Area

Collected Downriver and Upriver in 1992 . . . . . . . . . . . C-11

C-12 Reed Canarygrass Sample Results from 100-K Reactor Area

Collected Downriver and Upriver in 1992 . . . . . . . . . C-12

C-13 Reed Canarygrass Sample Results from 100-BC Reactor Area

Collected Downriver and Upriver in $1992 . . . . . . . . . . . . C-13$

D-1 Tree Leaf Sample Results Collected from 100 Areas in 1991 . . . . D-1

D-2 Tree Leaf Results Collected in July 1992 at 100 Area

Reactor Sites.................... D-3

D-3 Tree Leaf Results Collected in October 1992 . . . . . . . . . D-5

E-1 Coyote Results from 1992 . . . . . . . . . . . . . . . . E-1

E-2 Raptor Results from 1992 . . . . . . . . . . . . . . E-3

F-1 Ant Mound Soll Results at F Reactor from 1992 . . . . . . . . . . F-1

F-2 Ant Mound Results from H Reactor Collected in 1992 . . . . . . . . F-2

F-3 Ant Mound Results from D Reactor Collected in 1992 . . . . . . . . F-3

F-4 Ant Mound Results from $N$ Reactor in 1992 . . . . . . . . . . . . . F-4 


\section{LIST OF TABLES (cont.)}

F-5 Ant Mound Soll Results from BC Reactor in $1992 \ldots \ldots$. . . . . F-5

G-1 Burrow Mound Soll Results from F Reactor in $1992 \ldots$. . . . . . . G-1

G-2 Burrow Results from H Reactor Collected in $1992 \ldots \ldots$. . . . . . . G-2

G-3 Burrow Results from D Reactor collected in 1992 . . . . . . . . G-3

G-4 Burrow Mound Soll Results from K Reactor in 1992 . . . . . . . (a-4

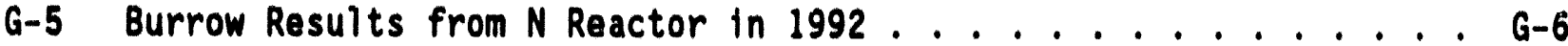

G-6 Burrow Mound So11 Results from BC Reactor in 1992 . . . . . . . G-7

H-1 Average Radionuclide Concentrations ( $\mathrm{pC} 1 / \mathrm{g}$ ) Detected in Soll

Samples near the 1301-N Liquid Waste Disposal Facility

from 1980 through $1991 \ldots \ldots$. . . . . . . . . . H-1

H-2 Average Radionuclide Concentrations ( $p C l / g$ ) Detected in 100 N Area Surface Soll Samples from 1980 through 1991 . . . . . . H-1

H-3 Average Radionuclide Concentrations ( $p C 1 / g$ ) Detected in 100-B/C Area Surface Soll Samples from 1981 to 1991 . . . . . . . . H-2

H-4 Average Radionuclide Concentrations ( $\mathrm{pC} / \mathrm{g}$ ) Detected in 100-D/DR Area Surface Soll Samples from 1981 to 1991 . . . . . . . H-2

H-5 Average Radionuclide Concentrations ( $\mathrm{pCi} / \mathrm{g}$ ) Detected in 100-F Area Surface Soil Samples from 1981 to 1991 . . . . . . . . . H-3

H-6 Average Radionuclide Concentrations ( $p C 1 / g$ ) Detected in 100-H Area Surface Soll Samples from 1981 to 1991 . . . . . . . . . H-3

H-7 Average Radionuclide Concentrations ( $\mathrm{pC} / \mathrm{g}$ ) Detected in 100-K Area Surface Soll Samples from 1981 to 1991 . . . . . . . . . H-4

H-8 200/600 Area Solls (pC1/g) . . . . . . . . . . . . H-4

H-9 300/400 Area Soll Sample $(p C i / g) \ldots \ldots$. . . . . . . . H-5

H-10 Average Radionuclide Concentrations ( $\mathrm{pCl} / \mathrm{g}$ ) Detected in Vegetation Samples near the 1301-N Liquid Waste Disposal Factlity from 1980 to $1991 \ldots \ldots$. . . . . . . . . H-5

H-11 Average Radionuclide Concentrations (pCi/g) Detected in 100-N Vegetation Samples from 1980 to 1991 . . . . . . . . H-6

H-12 Radionuclide Concentrations ( $\mathrm{pC} / \mathrm{g}$ ) Detected in N-Springs Vegetation Samples from 1980 to $1991 \ldots \ldots$. . . . . . . . H-6 


\section{LIST OF TABLES (cont.)}

$\mathrm{H}-13$ Average Radionuclide Concentrations ( $\mathrm{pCi} / \mathrm{g}$ ) Detected in 100-B/C Area Vegetation Samples from 1981 to 1991 . . . . . . . . H-7

$\mathrm{H}-14$ Average Radionuclide Concentrations ( $\mathrm{pC} i / g$ ) Detected in 100-D/DR Area Vegetation Samples from 1981 to 1991 . . . . . . H-7

H-15 Average Radionuclide Concentrations ( $\mathrm{pCi} / \mathrm{g}$ ) Detected in 100-F Area Vegetation Samples from 1981 to 1991 . . . . . . . H-8

H-16 Average Radionuclide Concentrations ( $\mathrm{pC} i / \mathrm{g}$ ) Detected in 100-H Area Vegetation Samples from 1981 to 1991 . . . . . . . . H-8

H-17 Average Radionuclide Concentrations ( $\mathrm{C} \mathrm{i} / \mathrm{g}$ ) Detected in 100-K Area Vegetation Samples from 1981 to 1991 . . . . . . . . H-9

$\mathrm{H}-18200$ Area Vegetation $(\mathrm{pCi} / \mathrm{g})$. . . . . . . . . . . . . . H-9

I-1 Species Observed in Vicinity of 100-BC Area ........... I-3

I-2 Species Observed Along Shoreline between 100-B and Allard Pumphouse ................... . . I-5

I-3 Species Observed between Allard Pumphouse and 100-K Area . . . . . I-7

I-4 Species Observed along Shoreline between 100-K and 100-N Areas.................... . . I-11

I-5 Species Observed in Dryland Area above Shoreline between $100-\mathrm{K}$ and 100-N Areas................. I-14

I-6 Species Observed Along Shoreline between 100-N and 100-D Areas..................... I- . . . . . . . .

I-7 Species Observed at Gravel/Cobble Shelf below 100-D Area . . . . I-18

I-8 Species Observed in Sandy Area East of 100-D Area . . . . . . . . I-20

I-9 Species Observed Downstream from 100-D Area, Boat Launch to River Mile 375 . . . . . . . . . . . . . . . . . I-21

I-10 Specie- Observed - River Nile 375 to 100-H Area . . . . . . . . . . I-23

I-11 Species Observed near 100-H Reactor . . . . . . . . . . . . I-26

I-12 Species Observed in the Vicinity of 100-F Area . . . . . . . . . . I-28 
WHE-EP-0620

This page intentionally left blank. 
WHC-EP-0620

\section{AREAS CERCLA ECOLOGICAL INVESTIGATIONS}

\subsection{INTRODUCTION}

Work plans establishing the tasks for conducting the Cumprehensive Environmental Response, Compensation, and Liability Act of 1980 (CERCLA) and Resource Conservation and Recovery Act of 1976 (RCRA) past-practice remedial investigations included ecological studies. For the 100 Areas, the ecological investigations are described identically in Appendix D-2 of each work $p l a n$ for groundwater operable units (i.e., 100-FR-3,100-HR-3,100-NR-2, 100-KR-4 and 100-BC-5) (e.g, DOE-RL 1992a). Three habitat types are found in each of these operable units: aquatic (the Columbia River), riparian, and terrestrial. The relative uniformity of these operable units permitted coordination of the ecological tasks, maximizing cost effectiveness and data usability. The ecological tasks identified in the work plans include data compllation, a preliminary ecological investigations report, threatened and endangered species protection, field activities, laboratory analysis, and data evaluation.

This document reports the results of the field activities tasks, including (1) vegetation, insect, bird, and mammal surveys; and (2) vegetation, coyote scat, raptor pellet, and small mammal and harvester ant burrow soil sampling. Sample analysis generally included metals, strontium-90, and gamma spectroscopy.

The results of the fiscal year (FY) 1991 ecological field activities were reported in Sackschewsky and Landeen (1992); some but not all of the data in that report are duplicated here. Other documents related to the 100 Areas ecological investigations include the following:

- Cushing, C. E., 1993, Aquatic Studies at the 100-HR-3 and 100-NR-1 Operable Units, PNL-8584, Pacific Northwest Laboratory, Richland, Washington

- Fitzner, R. E., S. G. Weiss, and J. A. Stegen, 1992, Biological Assessment for Threatened and Endangered Wildlife Species Related to CERCLA Characterization Activities, WHC-EP-0513, Westinghouse Hanford Company, Richland, Washington

- Fitzner, R. E., and S. G. Weiss, 1992, Bald Eagle Site Management Plan for the Hanford Site, South Central Washington, WHC-EP-0510, Westinghouse Hanford Company, Richland, Washington

- Landeen, D. S., 1992, Description of Work for 100 Areas Operable Unit Ecological Investigations, WHC-SD-EN-AP-090, Westinghouse Hanford Company, Richland, Washington

- Sackschewsky, M. R., 1992, Biological Assessment for Rare and Endangered Plant Species Related to CERCLA Characterization Activities, WHC-EP-0526, Westinghouse Hanford Company, Richland, Washington 
- Sackschewsky, M. R., D.S. Landeen, G. I. Baird, W. H. Rickard, and J. L. Downs, 1992, Vascular Plants of the Hanford Site, WHC-EP-0554, Westinghouse Hanford Company, Richland, Washington

- Stegen, J. A., 1992, Biological Assessment for State Candidate and Monitor Wildlife Species Related to CERCLA, WHC-SD-EN-EE-009 ENG, Westinghouse Hanford Company, Richland, Washington

- Weiss, S. G., and R. M. Mitchell, 1992, A Synthesis of Ecological Data from the 100 Areas of the Hanford Site, WHC-EP-0601, Westinghouse Hanford Company, Richland, Washington

- WHC, 1991a, Ecological Data Compllation Investigations Status Report for 100 Areas Operable Units, WHC-MR-0272, Westinghouse Hanford Company, Richland, Washington.

Other CERCLA ecological investigations al so have been conducted on the Hanford Site. The most significant of these relative to the 100 Areas was completed for the 300-FF-5 and 300-FF-1 operable units (e.g., Brandt et al. 1993, Brandt and Rickard 1992, Rickard et al. 1990, Thiede 1992). These documents cover both Columbia River and terrestrial surveys and sampling at the 300 Area. Other studies, such as the long-term Hanford Sitewide and the near-facility monitoring programs, are continuing to collect data useful for the CERCLA investigation (see Schmidt et al. 1992 and Woodruff et al. 1992).

\subsection{SCOPE OF WORK}

\subsection{OBJECTIVES}

The objectives of the ecological field investigations included the following:

- To provide a description of the flora and fauna associated with the 100 Areas operable units with an emphas is on (1) potentially significant pathways, and (2) those species that have been classified as threatened, endangered, candidate, or monitor species by the state and/or federal governments

- To evaluate existing concentrations of contaminants in major species and pathways assoctated with the 100 Areas operable units.

The information regarding contaminant uptake by biota may help identify indicator species that can be monitored to assess the effectiveness of remedial actions. The information regarding special state- or federaldesignated plant and wildi ife species also contribute in determining sitewide distributions of these species and present status in the 100 Areas. 
WHC-EP-0620

\subsection{FIELD INVESTIGATIONS}

Field investigations have concentrated on bird surveys, mammal and insect surveys, vegetation surveys, and sampling of various biota for radionuclides and inorganic waste constituents analysis. These surveys were completed in accordance with Appendix D-2 of the groundwater operable unit work plans (e.g., DOE-RL 1992a). Attention has also been given to plant and wildi ife species that have special classification status at state and/or federal levels. Table 1 lists and describes the various status codes for federal and state designations. The classifications of most concern are the federal and state threatened and endangered species. Table 2 contains the complete list of Hanford Site plants and animals that have special state or federal classification status and also indicates those species that were observed in the 100 Areas in 1991 and 1992. The Washington State classification codes in Table 1 were taken from the 1991 wildlife species 11sts (Washington Department of Wildilfe June 19, 1991) and plant species IIsts (Washington Natural Heritage Program 1990). The federal species designations were obtained from the 50 CFR 17, which is updated several times each year. Both federal and state lists are revised frequently.

\subsection{BIRD SURVEYS}

\subsection{BIRD SURVEYS}

Three winter wildlife surveys were conducted (December 1990 and January and February 1991) at the 100-HR-3 and 100-BC-5 operable units. The main purposes were to help verify existing species lists (e.g., Landeen et al. 1992, Fitzner and Gray 1992) for the 100 Areas, to Identify potentially significant pathways, and to verify and document the spectes of special concern that use the operable units, such as the American white pelican, bald eagle, and peregrine falcon.

All bird spectes observed during the surveys are listed in Table 3 , in addition to those species observed at other times of the year. Some of the bird species commonly observed in the winter included Canada goose, horned lark, white-crowned sparrow, common raven, starling, great blue heron, and rock dove. Bald eagles and white pelicans were observed on all three surveys, but peregrine falcons were not observed. However, peregrine falcons have been documented to fly through the Hanford Site during migration and have been sighted flying along the Columbia River (Landeen et al. 1992). There were at least two peregrine falcon sightings at the Hanford Site in Aprt1 1992 and January 1993.

Spring, summer, and fall bird surveys also were conducted with in the $100-H R-3$ and 100-BC-5 operable units. Surveys were conducted within 1 hour of sunrise, in accordance with Westinghouse Hanford Company (Westinghouse Hanford) procedure EII 5.3 (WHC 1991b), on April 5; May 6, 24, and 29; and June 7,11 , and 27, 1991. Other bird surveys conducted at various times during the day occurred on March 4 and 8 ; Apri1 16, 19, 22, 23, and 24; May 14, 22, and 24; June 27; and September 11 and 20, 1991. Bird species 
observations also were recorded when other field work was being conducted throughout all seasons in 1991 and 1992. Surveys in the riparian zones along the Columbia River were conducted at all the reactors by walking within $50 \mathrm{~m}$ of the high-water mark along the river. Surveys also were conducted by walking and driving within the fenced areas at $100-B,-C,-H,-D$, and -DR reactors. All bird species seen or heard on these occasions were recorded. No effort was made to quantify bird species inhabiting the operable units.

Table 3 also indicates those birds observed in breeding and nesting activities and those birds observed within the fenced areas at the 100 Areas reactors. Game birds observed include mourning doves, ring-necked pheasants, California quall, and gray partridge. The bird surveys, while not re-documenting the presence of all species on prevlous ilsts, did verify these lists in establishing that no potentially significant (in terms of contaminant pathways) "new" species were recorded.

\subsection{POTENTIALLY SIGNIFICANT BIRD SPECIES}

Table 3 includes birds that are both common on the Hanford site (under status) and birds that were observed in the reactor areas. These birds generally represent five feeding types (depending on the season):

- Consumers of flying insects--common nighthawks, eastern and western kingbirds, flycatcher sp., swallow sp.

- Consumers of ground insects--killideer, American robin

- Predators and/or scavengers--ring-billed gull, California gull, American kestrels, black-billed magpie, common raven, loggerhead shrike

- Seed-eaters--white-crowned sparrows, mourning and rock doves, quail, dark-eyed juncos, house finch

- Consumers of seeds and predominately surface insects--horned larks, western meadowlark, red-winged and Brewer's blackbirds, European starling, song sparrow, house sparrow.

In addition, common aquatic and riparian birds feed on shoreline vegetation and river biota. Those 1 isted below are also common Hanford Site birds, seen during the surveys on and along the river. These may be broken into the following two general feeding groups:

- Consumers of aquatic insects and fish--bufflehead, common merganser, great blue heron, American white pelican, bald eagles

- Consumers of aquatic and terrestrial vegetation--Canada goose, mallard, American wigeon, northern shoveler, gadwall, redhead.

From this listing of commonly observed birds and their feeding habits, an evaluation of their significance relative to contaminant transport can be made (Section 5.0). 
Table 1. Federal and State Status Codes for Special Plants and Animals. (sheet 1 of 2)




Table 1. Federal and State Status Codes for Special Plants and Animals. (sheet 2 of 2)

\begin{tabular}{|c|c|}
\hline Code & Explanation \\
\hline \multicolumn{2}{|r|}{ State (plants) } \\
\hline SE & $\begin{array}{l}\text { Endangered. This status is assigned to each vascular plant taxon } \\
\text { in danger of becoming ext inct or extirpated in Washington State in } \\
\text { the near future if factors contributing to its decline continue. } \\
\text { Populations of these taxa are at critically low levels or their } \\
\text { habitats have been degraded or depleted to a significant degree. }\end{array}$ \\
\hline ST & $\begin{array}{l}\text { Threatened. A threatened vascular plant taxon likely to become } \\
\text { endangered in the near future in Washington State if factors } \\
\text { contributing to its population decline or habitat degradation or } \\
\text { loss continue. }\end{array}$ \\
\hline SS & $\begin{array}{l}\text { Sensitive. A vascular plant taxon is labelled sensitive when it } \\
\text { is vulnerable or declining and could become endangered or } \\
\text { threatened in Washington State without active management or } \\
\text { removal of threats. }\end{array}$ \\
\hline EX & $\begin{array}{l}\text { Possibly extinct or extirpated in Washington State. Based on } \\
\text { recent field searches, several plant taxa are considered to be } \\
\text { possibly extinct or extirpated in Washington state. Taxa in this } \\
\text { group are all high priorities for fleld investigation. If found, } \\
\text { they will be assigned one of the above status categories. }\end{array}$ \\
\hline$S M_{1}$ & $\begin{array}{l}\text { Monftor Group 1. Taxa for which there is insufficlent data to } \\
\text { support listing as threatened, endangered, or sensitive. }\end{array}$ \\
\hline $\mathrm{SM}_{2}$ & Monitor Group 2. Taxa with unresolved taxonomic questions. \\
\hline$S M_{3}$ & $\begin{array}{l}\text { Monitor Group 3. Taxa that are more abundant and/or less } \\
\text { threatened than previously assumed. }\end{array}$ \\
\hline
\end{tabular}


政

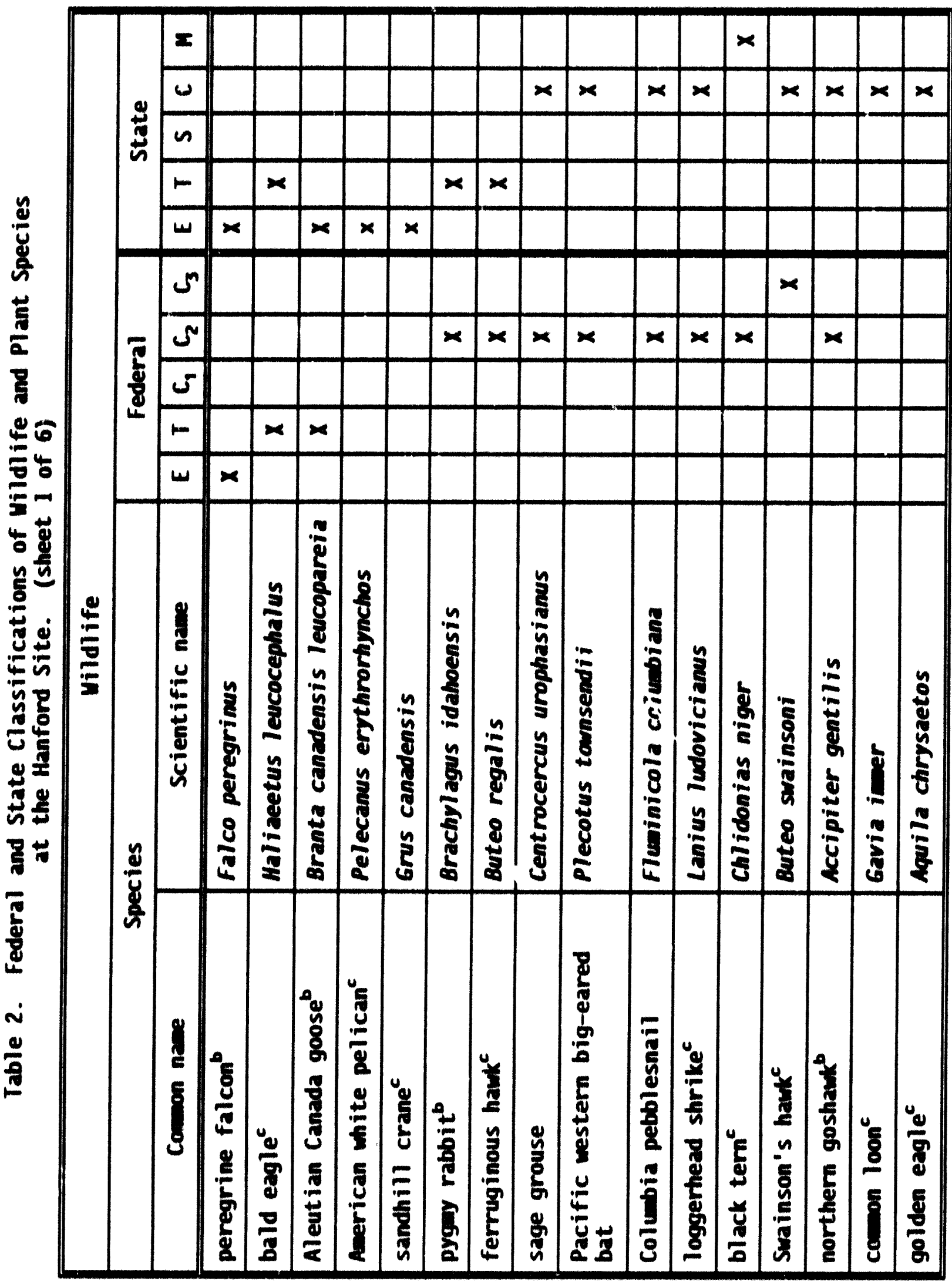




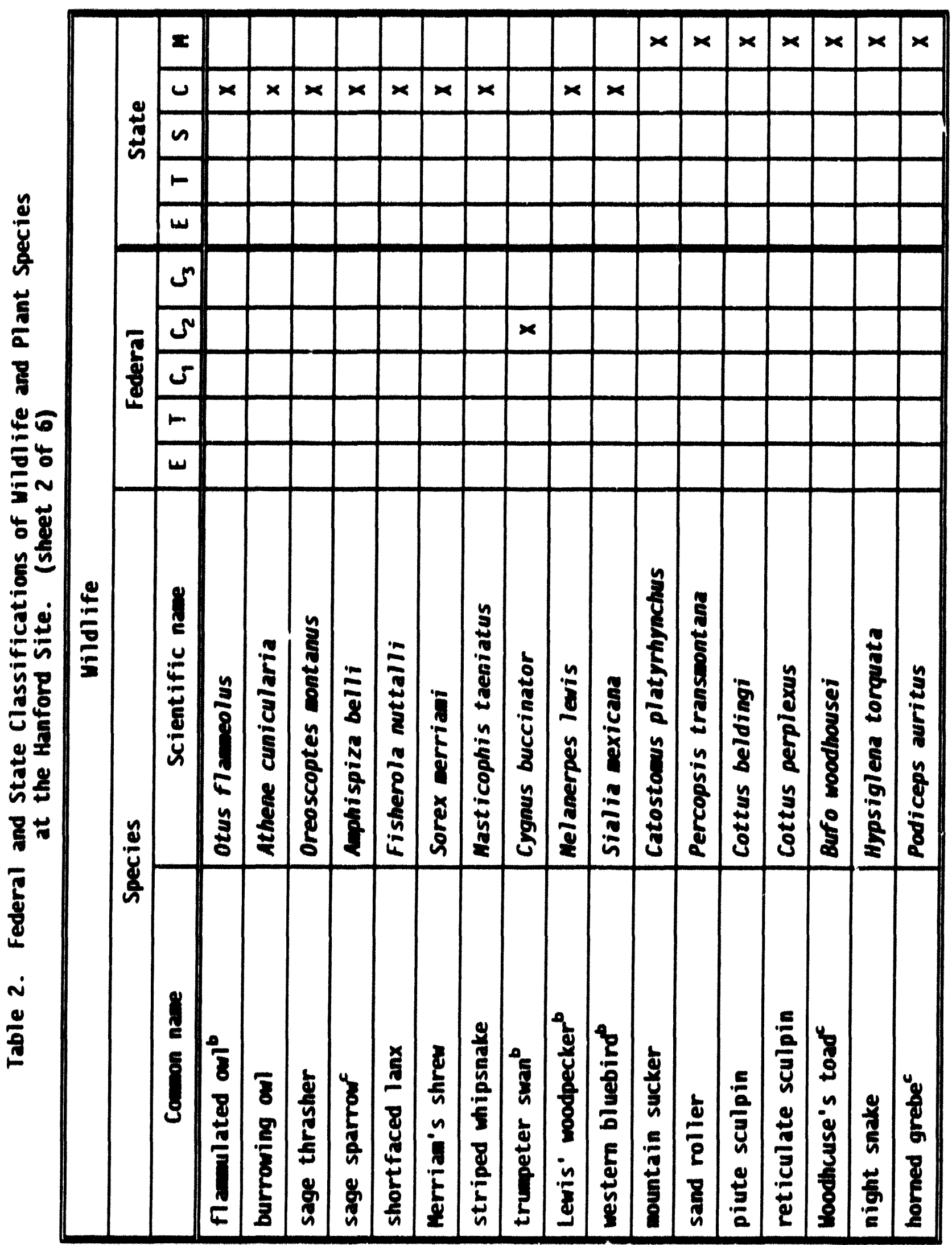


WHC-EP-0620

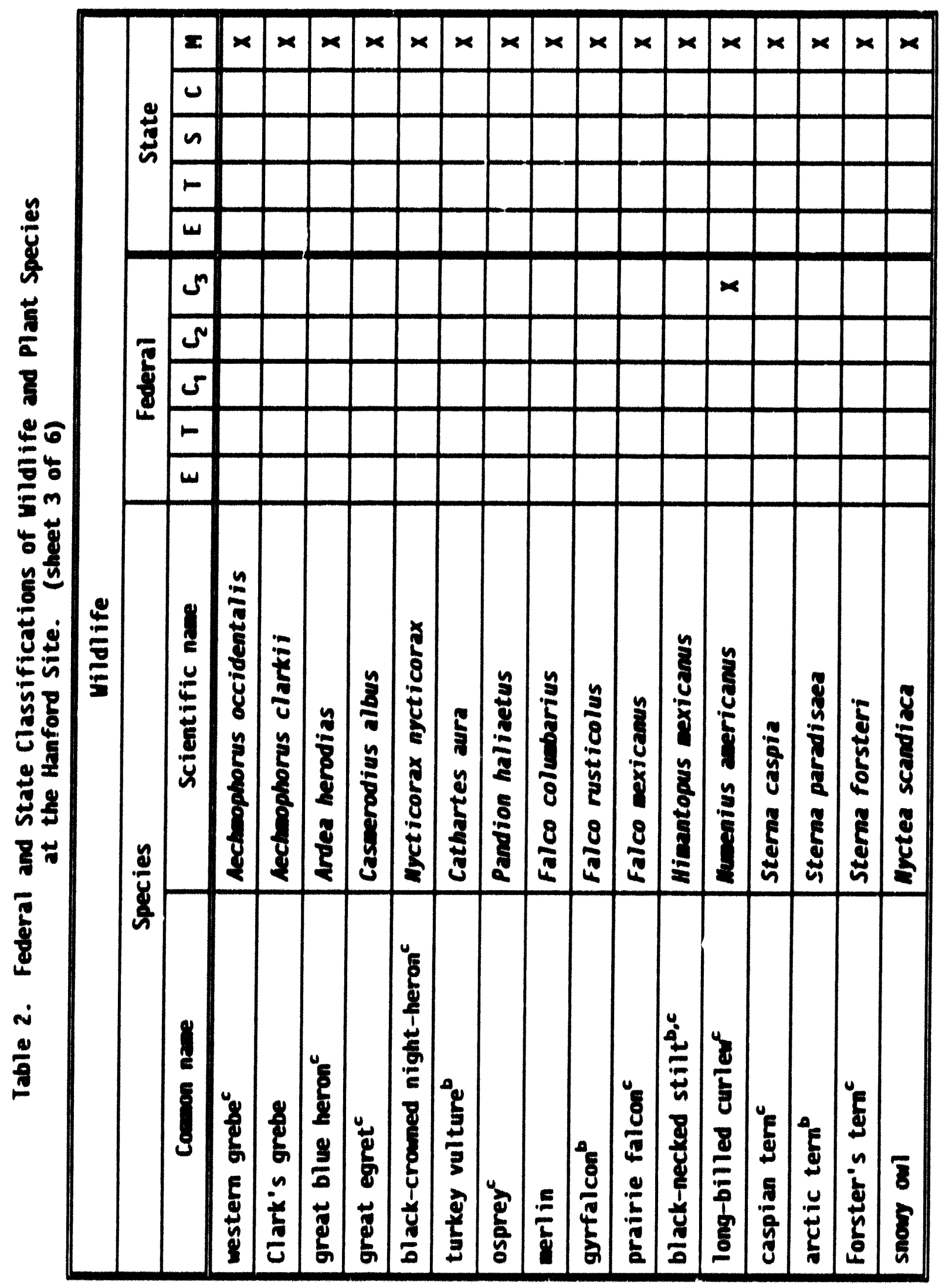




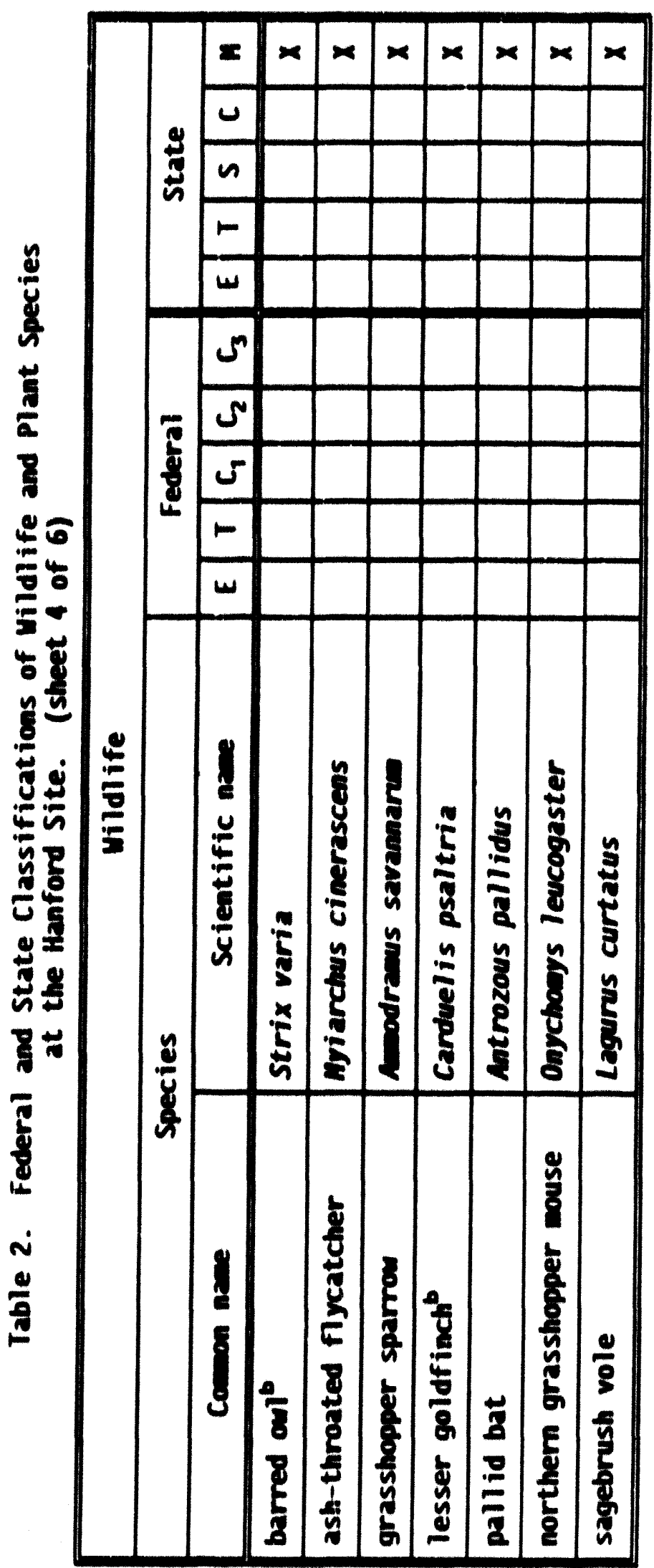




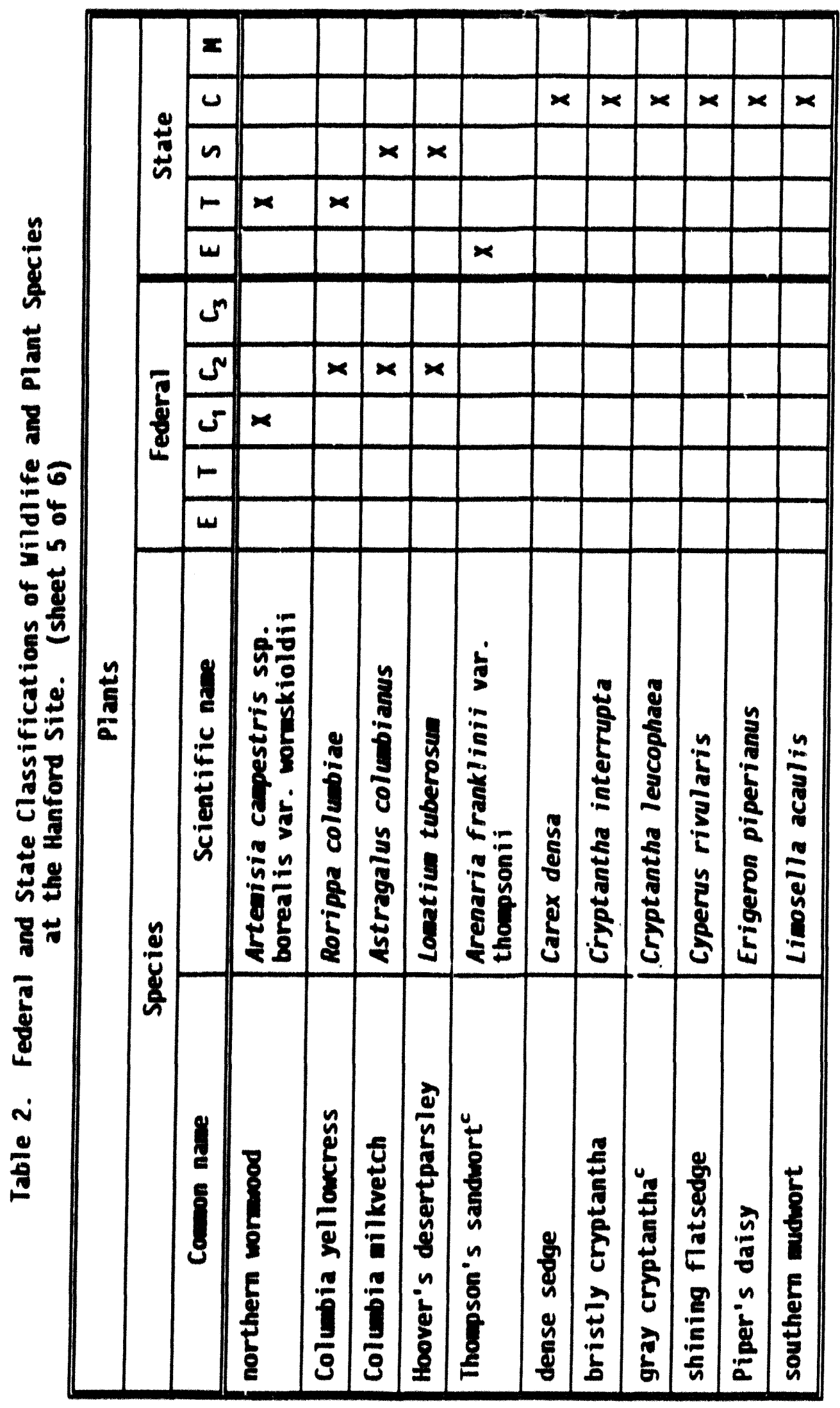







Table 3. Birds Observed at 100 Areas Operable Units. (sheet 1 of 4 )

\begin{tabular}{|c|c|c|c|}
\hline Family & Common name & Genus species & Status \\
\hline Gavilidae & common loon & Gavia immer & Rw \\
\hline Podicipedidae & $\begin{array}{l}\text { pied-billed grebe } \\
\text { horned grebe } \\
\text { western grebe }\end{array}$ & $\begin{array}{l}\text { Podilymbus podiceps } \\
\text { Podiceps auritus } \\
\text { Aechmophorus occidentalis }\end{array}$ & $\begin{array}{l}\text { Cr } \\
\text { Uw } \\
\text { Ur }\end{array}$ \\
\hline Pelecanidae & $\begin{array}{l}\text { American white } \\
\text { pelican }\end{array}$ & Erythrorhynchos pelecanus & $\mathrm{Cr}$ \\
\hline Phalacrocoracidae & $\begin{array}{l}\text { double-crested } \\
\text { cormorant }\end{array}$ & Phalacrocorax auritus & $\mathrm{Rr}$ \\
\hline Ardeidae & $\begin{array}{l}\text { great blue heron } \\
\text { black-crowned } \\
\text { nignt-heron } \\
\text { great egret }\end{array}$ & $\begin{array}{l}\text { Ardea herodias } \\
\text { Nycticorax nycticorax } \\
\text { Casmerodius albus }\end{array}$ & $\begin{array}{l}\mathrm{Cr} \\
\mathrm{Cr} \\
\mathrm{Rm}\end{array}$ \\
\hline Anatidae & $\begin{array}{l}\text { Canada goose } \\
\text { tundra swan } \\
\text { mallard } \\
\text { northern pintail } \\
\text { blue-winged teal } \\
\text { cinnamon teal } \\
\text { northern shoveler } \\
\text { gadwall } \\
\text { American wigeon } \\
\text { redhead } \\
\text { ring-necked duck } \\
\text { lesser scaup } \\
\text { greater scaup } \\
\text { common goldeneye } \\
\text { bufflehead } \\
\text { common merganser } \\
\text { ruddy duck }\end{array}$ & $\begin{array}{l}\text { Branta canadensis } \\
\text { Cygnus columbianus } \\
\text { Anas platyrhynchos } \\
\text { Anas acuta } \\
\text { Anas discors } \\
\text { Anas cyanoptera } \\
\text { Anas clypeata } \\
\text { Anas strepera } \\
\text { Anas americana } \\
\text { Aythya americana } \\
\text { Aythya collaris } \\
\text { Aythya affinis } \\
\text { Aythya marila } \\
\text { Bucephala clangula } \\
\text { Bucephala albeola } \\
\text { Mergus merganser } \\
\text { Oxyura jamaicensis }\end{array}$ & 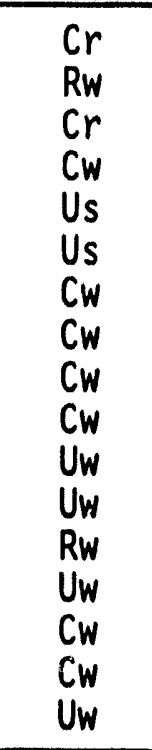 \\
\hline Accipitridae & $\begin{array}{l}\text { osprey } \\
\text { bald eagle } \\
\text { northern harrier } \\
\text { Swainson's hawk } \\
\text { red-tailed hawk } \\
\text { ferruginous hawk } \\
\text { rough-legged hawk } \\
\text { golden eagle }\end{array}$ & $\begin{array}{l}\text { Pandion haliaeetus } \\
\text { Haliaeetus leucocephalus } \\
\text { Circus cyaneus } \\
\text { Buteo swainsoni } \\
\text { Buteo jamaicensis } \\
\text { Buteo regalis } \\
\text { Buteo lagopus } \\
\text { Aquila chrysaetos }\end{array}$ & $\begin{array}{l}\text { Um } \\
C w \\
C r \\
\text { Us } \\
C r \\
\text { Rs } \\
\text { Rw } \\
\text { Ur }\end{array}$ \\
\hline Falconidae & $\begin{array}{l}\text { American kestrel }{ }^{a, b} \\
\text { merlin } \\
\text { pratrie falcon }\end{array}$ & $\begin{array}{l}\text { Falco sparverius } \\
\text { Falco columbarius } \\
\text { Falco mexicanus }\end{array}$ & $\begin{array}{l}\mathrm{Cr} \\
\mathrm{Rr} \\
\mathrm{Ur}\end{array}$ \\
\hline
\end{tabular}


Table 3. Birds Observed at 100 Areas Operable Untts.

(sheet 2 of 4 )

\begin{tabular}{|c|c|c|c|}
\hline Family & Common name & Genus species & Status \\
\hline Phasianidae & $\begin{array}{l}\text { gray partridge } \\
\text { chukar } \\
\text { ring-necked } \\
\text { pheasant } \\
\text { california quail }\end{array}$ & $\begin{array}{l}\text { Perdix perdix } \\
\text { Alectoris chukar } \\
\text { Phasianus colchicus } \\
\text { Callipepla californica }\end{array}$ & $\begin{array}{l}\text { Ur } \\
\text { Ur } \\
U_{r} \\
\text { Ur }\end{array}$ \\
\hline Rallidae & American $\operatorname{coot}^{\circ}$ & Fulica americana & $\mathrm{Cr}$ \\
\hline Gruidae & sandhill crane & Grus canadensis & Um \\
\hline Charadritdae & killdeer ${ }^{a, b}$ & Charadrius vociferus & $\mathrm{Cr}$ \\
\hline Scolopacidae & $\begin{array}{l}\text { greater yellowlegs } \\
\text { long-billed curlew } \\
\text { common snipe }\end{array}$ & $\begin{array}{l}\text { Tringa mel anol euca } \\
\text { Numenius americanus } \\
\text { Gallinago gallinago }\end{array}$ & $\begin{array}{l}\text { Um } \\
\text { Cs } \\
\text { Ur }\end{array}$ \\
\hline Laridae & $\begin{array}{l}\text { ring-billed gull } \\
\text { california gull } \\
\text { caspian tern } \\
\text { Forster's tern } \\
\text { black tern }\end{array}$ & $\begin{array}{l}\text { Larus delawarensis } \\
\text { Larus cal ifornicus } \\
\text { Sterna caspia } \\
\text { Sterna forsteri } \\
\text { Chlidonias niger }\end{array}$ & $\begin{array}{l}\mathrm{Cr} \\
\mathrm{Cr} \\
\mathrm{Us} \\
\mathrm{Cs} \\
\mathrm{Rm}\end{array}$ \\
\hline Columbidae & $\begin{array}{l}\text { rock dove } e^{a, b} \\
\text { mourning dove }\end{array}$ & $\begin{array}{l}\text { Columba livia } \\
\text { Zenaida macroura }\end{array}$ & $\begin{array}{l}\mathrm{Cr} \\
\mathrm{Cr}\end{array}$ \\
\hline Tytonidae & common barn-ow $7^{b}$ & Tyto alba & Ur \\
\hline Strigidae & $\begin{array}{l}\text { great horned owl } \\
\text { long-eared ow }{ }^{\text {a }}\end{array}$ & $\begin{array}{l}\text { Bubo virginianus } \\
\text { Asio otus }\end{array}$ & $\begin{array}{l}\text { Ur } \\
\text { Ur }\end{array}$ \\
\hline Caprimulgidae & common nighthawk & Chordeiles minor & Cs \\
\hline Trochilidae & calliope hummingbird & Stellula calliope & Um \\
\hline Alcedinidae & belted kingfisher & Ceryle alcyon & Ur \\
\hline Picidae & northern flicker & Colaptes auratus & $\mathrm{Cr}$ \\
\hline Tyrannidae & $\begin{array}{l}\text { western wood-pewee } \\
\text { willow flycatcher } \\
\text { Say's phoebe } \\
\text { western kingbird } \\
\text { eastern kingbird }\end{array}$ & $\begin{array}{l}\text { Contopus sordidulus } \\
\text { Empidonax traillii } \\
\text { Sayornis saya } \\
\text { Tyrannus verticalis } \\
\text { Tyrannus tyrannus }\end{array}$ & $\begin{array}{l}\text { Um } \\
\text { Rm } \\
\text { Us } \\
\text { Cs } \\
\text { Us }\end{array}$ \\
\hline Al audidae & horned lark ${ }^{a, b}$ & Eremophila alpestris & $\mathrm{Cr}$ \\
\hline Hirundinidae & $\begin{array}{l}\text { northern rough- } \\
\text { winged swallow } \\
\text { bank swallow } \\
\text { cliff swallowa,b } \\
\text { barn swall owa,b }\end{array}$ & $\begin{array}{l}\text { Stelgidopteryx } \\
\text { serripennis } \\
\text { Riparia riparia } \\
\text { Hirundo pyrrhonota } \\
\text { Hirundo rustica }\end{array}$ & $\begin{array}{l}\text { Us } \\
\text { Us } \\
\text { Cs } \\
\text { Cs }\end{array}$ \\
\hline
\end{tabular}


Table 3. Birds Observed at 100 Areas Operable Units. (sheet 3 of 4 )

\begin{tabular}{|c|c|c|c|}
\hline Family & Common name & Genus species & Status \\
\hline Corvidae & $\begin{array}{l}\text { black-billed } \\
\text { magp le,b } \\
\text { common raven }{ }^{a, b} \\
\text { Clark's nutcracker }\end{array}$ & $\begin{array}{l}\text { Pica pica } \\
\text { Corvus corax } \\
\text { Nucifraga columbiana }\end{array}$ & $\begin{array}{l}\mathrm{Cr} \\
\mathrm{Cr} \\
\mathrm{Am}\end{array}$ \\
\hline Paridae & $\begin{array}{l}\text { black-capped } \\
\text { chickadee }\end{array}$ & Parus atricapillus & Ur \\
\hline Troglodytidae & marsh wren" & Cistothorus palustris & Ur \\
\hline Muscicapidae & $\begin{array}{l}\text { ruby-crowned kinglet } \\
\text { American robin }{ }^{\circ, b} \\
\text { varied thrush }\end{array}$ & $\begin{array}{l}\text { Regulus calendula } \\
\text { Turdus migratorius } \\
\text { Ixoreus naevius }\end{array}$ & $\begin{array}{l}\text { Uw } \\
\text { Cr } \\
\text { Uw }\end{array}$ \\
\hline Bombyc 1111 dae & cedar waxwing & Bombyctlla cedrorum & Ur \\
\hline Lanilidae & $\begin{array}{l}\text { northern shrike } \\
\text { loggerhead shrike }\end{array}$ & $\begin{array}{l}\text { Lanius excubitor } \\
\text { Lanius ludoviclanus }\end{array}$ & $\begin{array}{l}\text { UW } \\
\text { US }\end{array}$ \\
\hline Sturnidae & European starling ${ }^{a, b}$ & Sturnus vulgaris & $\mathrm{Cr}$ \\
\hline Vireonidae & $\begin{array}{l}\text { solitary vireo } \\
\text { warbling vireo }\end{array}$ & $\begin{array}{l}\text { Vireo solitarius } \\
\text { Vireo gilvus }\end{array}$ & $\begin{array}{l}U_{m} \\
U_{m}\end{array}$ \\
\hline Emberizidae & $\begin{array}{l}\text { yellow warbler } \\
\text { yellow-rumped } \\
\text { warbler } \\
\text { Townsend's warbler } \\
\text { Wilson's warbler } \\
\text { western tanager } \\
\text { black-headed } \\
\text { grosbeak } \\
\text { vesper sparrow } \\
\text { lark sparrow } \\
\text { sage sparrow } \\
\text { savannah sparrowa,b } \\
\text { song sparrow } \\
\text { white-crowned } \\
\text { sparrow } \\
\text { dark-eyed juncob } \\
\text { red-winged } \\
\text { blackbird,b } \\
\text { western meadowlarka,b } \\
\text { yellow-headed } \\
\text { blackbird } \\
\text { Brewer's blackbirda,b } \\
\text { brown-headed cowbird } \\
\text { northern oriole }\end{array}$ & $\begin{array}{l}\text { Dendroica petechia } \\
\text { Dendroica coronata } \\
\text { Dendroica townsendi } \\
\text { Wilsonia pusilla } \\
\text { Piranga ludoviciana } \\
\text { Pheucticus melanocephalus } \\
\text { Pooecetes gramineus } \\
\text { Chondestes grammacus } \\
\text { Amphispiza belli } \\
\text { Passerculus sandwichensis } \\
\text { Melospiza melodia } \\
\text { Zonotrichia leucophrys } \\
\text { Junco hyemalis } \\
\\
\text { Agelaius phoeniceus } \\
\text { Sturnella neglecta } \\
\text { Xanthocephalus } \\
\text { xanthocephalus } \\
\text { Euphagus cyanocephalus } \\
\text { Molothrus ater } \\
\text { Icterus galbula }\end{array}$ & $\begin{array}{l}\text { Us } \\
C W \\
\text { Um } \\
\text { Um } \\
\text { Um } \\
\text { Us } \\
\text { Rm } \\
\text { Rs } \\
\text { Us } \\
\text { Us } \\
C r \\
C r \\
C W \\
C r \\
C r \\
C s \\
C r \\
C r \\
C s\end{array}$ \\
\hline
\end{tabular}


Table 3. Birds Observed at 100 Areas Operable Units.

(sheet 4 of 4 )

\begin{tabular}{|l|l|l|c|}
\hline \multicolumn{1}{|c|}{ Family } & \multicolumn{1}{|c|}{ Common name } & \multicolumn{1}{c|}{ Genus species } & Status \\
\hline Fringlllidae & $\begin{array}{l}\text { house finch } \\
\text { American goldfinch }\end{array}$ & $\begin{array}{l}\text { Carpodacus mexicanus } \\
\text { Carduelis tristis }\end{array}$ & $\begin{array}{c}\text { Cr } \\
\text { Ur }\end{array}$ \\
\hline Passeridae & house sparrow & Passer domesticus & Cr \\
\hline
\end{tabular}

A status rating is given for abundance and seasonal occurrence for each specles as follows: Abundences

$C$ - common; often seen or heard In appropriate habltat.

U uncomion; usully present but not always seen or heard.

- reres prosent in epproprlate habitats only in small numbers, seldom seen or heard.

A - ecefdental; appeared once or twlce, but well out of normal renge.

seasonal occurrence:

$r$ - resident; present all year but abundance may very sensonally.

- sumar vieltor (includes epring and fall).

- winter visltor (includes epring and fall).

ro migrent.

P that were observed in breeding and nesting activities.

bspecies observed within the fenced areas surrounding the 100 Arese reactors. 
WHC-EP-0620

\subsection{INSECT AND MAMMAL SURVEYS}

All mammal species observed during field work activities (such as vegetation surveys, bird surveys, sampiing, and general site reconnalssance at the operable units) were recorded. No effort was made to quant ify mammals inhabiting these sites or to inventory bat species that might be present. Trapping was not conducted to determine the presence or absence of small mammal species. Harvester ant colonies were recorded at individual waste sites and burtal grounds assoctated with the reactors.

\subsection{MAMHALS}

All mammals observed inhabiting the operable units were recorded. Signs of animal activity such as burrowing, tracks, and scat were accepted as evidence that the animal was inhabiting or using a given area. For instance, badgers were never sighted, but ample evidence of burrowing activity in several areas indicated that badgers do inhabit or forage for prey throughout the 100 Areas. Mammals observed on several occastons included coyotes, mule deer, blacktall jackrabbits, and porcupines. Burrowing evidence of small mamnals such as the Great Bas in pocket mouse and northern pocket gopher was also common. Table 4 lists all the mammals observed during the study. Table 4 also indicates those mammals known to inhabit the 100 Areas but not actually observed during the course of the study (Rickard et al. 1974).

Of these mammals, the mule deer, coyote, Great Bas in pocket mouse, jackrabbit and cottontall rabbit are the most common and most likely to be significant pathways for contaminants. The following dietary information is taken from Greager (1981).

Mule deer depend heavfly on the riparian vegetation during the hot, dry summer months when other plants have dried. These deer eat willows, Russtan thistle, goldenrod, and other plants but tend to avold gray rabbitbrush and cheatgrass.

Jackrabbits consume predominately yarrow, turpentine cymopterus, Jim $\mathrm{HIIl}$ mustard, buckwheat, and rabbitbrush; cottontall rabbits feed on seasonally avallable herbs and shrubs.

Coyotes are omnivorous, eating whatever may be most plentiful and easiest to catch, such as rabbits, pocket mice, grasshoppers, darkling beetles, birds, deer, reptiles, fruit, and grasses.

The single most important food item of pocket mice is cheatgrass seeds, with insects and spiders preferred in spring before seeds ripen.

\subsection{MAMMAL AND INSECT SURVEYS AT INDIVIDUAL WASTE SITES}

As previously mentioned, insect and mammal surveys were conducted at individual waste sites (trenches, cribs, burial grounds, etc.) associated with the reactors as identified in Stone (1989). Burrowing activity by small mammals was observed and recorded, as was the presence of harvester ant 
mounds. Harvester ants excavate matertals and bring them to the surface from as far as 15 to $20 \mathrm{ft}$ deep and have been implicated in bringing up contamination from some of the burial grounds in the 200 East Area (Conkiln et al. 1985). At the Hanford site, ants are probably the insects that are most likely to bring up any significant amounts of contaminated material.

The majority of the trenches, cribs, and burial grounds in these operable units have been covered with large amounts of cobble and treated with nonselective herbicides for several years; so, few of the waste sites have flora or fauna inhabiting them. When vegetation is encountered, tumbleweed is predominant at these sites. Results of the surveys taken at these waste sites are provided in Table 5.

\subsection{WILDLIFE SURVEY CONCLUSIONS}

The wildilfe species that were observed in the 100 Areas verifled previous published lists even though all specles known to inhabit the 100 Areas were not actually observed. No new spectes were documented that would be of significance in an ecological assessment.

Intrustve activitles, such as well drilling, that are conducted inside the controlled-area fences at the operable units will not have any significant negative impact on wildlife. Well drilling and cleanup/remedial activities that are conducted outside the fences will have minimal impact on wildiffe if the recommendations contained in the three documents described below are followed. These documents are the Bald Eagle Management Plan (Fitzner and Weiss 1992), the Biological Assessment of Threatened and Endangered Species (Fitzner et al. 1992), and the Blological Assessment for State Cardidate and Monitor species (Stegen 1992). DOE and WHC policy also states that sitespecific ecological surveys will be conducted at all sites where cleanup and remedial actions are performed. These site-specific surveys also provide recommendations that can mitigate impacts to wildiffe.

While this report is not intended to be an ecological risk assessment, a preliminary, qualitative, evaluation of the significance of the potentlal wildife pathways is provided. This information may be useful for future sampling efforts, risk assessors, and risk managers.

The consumers of flying insects indicated in Section 3.1 are not expected to consume significant contamination in their prey, because the consumers and their prey are both highly moblle. In addition, current and prevtous work by Cushing (1993 and Cushing et al. 1981) indicates no measurable contamination in aquatic insect larvae, which presumably would have a higher body burden than the flying adults. However, mud-nest bullding behavior in swallows may be a transport pathway if the swallows use contaminated mud for nest construction. No evidence of this was seen in the 100 Areas.

Members of the other groups 11 sted previously have greater potentlal for significant uptake of contaminants. However, the results of near-field 
Table 4. List of All the Mammals Observed and Known To Inhabit the 100 Areas Operable Units.

\begin{tabular}{|c|c|}
\hline Common name & Sctentific name \\
\hline $\begin{array}{l}\text { badger } \\
\text { coyote } \\
\text { Great Basin pocket mouse } \\
\text { northern pocket gopher } \\
\text { beaver } \\
\text { blacktall jackrabbit } \\
\text { bushytall woodrat } \\
\text { mountain cottontall } \\
\text { mule deer } \\
\text { porcupine } \\
\text { muskrat }\end{array}$ & $\begin{array}{l}\text { Taxidea taxus } \\
\text { Canis latrans } \\
\text { Perognathus parvus } \\
\text { Thomomys talpoides } \\
\text { Castor canadensis } \\
\text { Lepus callfornicus } \\
\text { Neotoma cinerea } \\
\text { Sylvilagus nuttalli } \\
\text { Odocolleus hemionus } \\
\text { Erethizon dorsatum } \\
\text { Ondatra zibethica }\end{array}$ \\
\hline \multicolumn{2}{|c|}{$\begin{array}{c}\text { Mammals known to inhablt areas near the Columbia River } \\
\text { but not observed }\end{array}$} \\
\hline $\begin{array}{l}\text { vagrant shrew } \\
\text { palijd bat } \\
\text { itttle brown myot Is } \\
\text { yuma myot is } \\
\text { townsend ground squirrel } \\
\text { western harvest mouse } \\
\text { deer mouse } \\
\text { sagebrush vole } \\
\text { montane meadow mouse } \\
\text { Nomway rat } \\
\text { house mouse } \\
\text { racoon } \\
\text { mink } \\
\text { longtail weasel } \\
\text { shorttall weasel } \\
\text { otter } \\
\text { striped skunk } \\
\text { bobcat }\end{array}$ & $\begin{array}{l}\text { Sorex vagrans } \\
\text { Antrozus pallidus } \\
\text { Myotis lucifugus } \\
\text { Myot is yumanensis } \\
\text { Spermophilus townsendif } \\
\text { Reithrodontomys megalotis } \\
\text { Peromyscus maniculatus } \\
\text { Lagurus curtatus } \\
\text { Microtus montanus } \\
\text { Rattus norvegicus } \\
\text { Mus musculus } \\
\text { Procyon lotor } \\
\text { Mustela vison } \\
\text { Mustela frenata } \\
\text { Mustela erminea } \\
\text { Lutra candensis } \\
\text { Mephitis mephitis } \\
\text { Lynx rufus }\end{array}$ \\
\hline
\end{tabular}


Table 5. Insect and Mammal Surveys at 100 Areas Operable Units. (sheet 1 of 3 )

\begin{tabular}{|c|c|c|}
\hline $\begin{array}{l}\text { Reactor } \\
\text { site }\end{array}$ & Waste stte & Comments \\
\hline$B C$ & $116-8-1$ (trench) & All cobble, some tumbleweeds \\
\hline$B C$ & $116-8-5(\mathrm{cr} \mid \mathrm{b})$ & $\begin{array}{l}\text { Chain-link fence, big tumbleweeds, } \\
\text { deer tracks, pocket mouse activity }\end{array}$ \\
\hline$B C$ & $\begin{array}{l}116-B-7 \\
\text { (outfall structure) }\end{array}$ & $\begin{array}{l}\text { Partial chain-link fence, lots of } \\
\text { tumbleweeds }\end{array}$ \\
\hline$B C$ & $\begin{array}{l}\text { 116-8-8 } \\
\text { (outfall structure) }\end{array}$ & $\begin{array}{l}\text { Three ant mounds (2 large), deer } \\
\text { tracks, pocket mouse activity, } \\
\text { cheatgrass, tumbleweeds, and } \\
\text { rabbltbrush }\end{array}$ \\
\hline$B C$ & $\begin{array}{l}116-8-11 \\
\text { (rotention basin) }\end{array}$ & $\begin{array}{l}\text { Chain-link fence, all cobble, } \\
\text { a few tumbleweeds }\end{array}$ \\
\hline$B C$ & $\begin{array}{l}116-C-5 \\
\text { (rotention basin) }\end{array}$ & Two tanks, all cobble \\
\hline$B C$ & 118-8-2 (burtal ground) & All cobble and tumbleweeds \\
\hline $\mathbf{D}$ & 116-DR-1 (trench) & Cobble and tumbleweeds \\
\hline D & $\begin{array}{l}116-D R-5 \\
\text { (outfall structure) }\end{array}$ & $\begin{array}{l}\text { Some rabbltbrush, three ant } \\
\text { mounds, rabbit sign }\end{array}$ \\
\hline 0 & 116-0-3 (french draln) & Cobble and tumbleweeds \\
\hline D & 116-0-4 (french drain) & Cobble and tumbleweeds \\
\hline D & $\begin{array}{l}116-0-5 \\
\text { (outfall structure) }\end{array}$ & Some rabbltbrush, rabbit sign \\
\hline D & $\begin{array}{l}116-0-7 \\
\text { (retention bas in) }\end{array}$ & $\begin{array}{l}\text { Chain-link fence, cobble and } \\
\text { tumbleweeds }\end{array}$ \\
\hline 0 & 116-D-1A (trench) & Cobble and tumbleweeds \\
\hline D & 116-D-18 (trench) & Cobble and tumbleweeds \\
\hline D & 118-D-1 (burial ground) & All cobble, no vegetation \\
\hline 0 & 118-0-2 (burial ground) & Cobble and tumbleweeds \\
\hline D & 118-0-3 (burtal ground) & Cobble and tumbleweeds \\
\hline D & 120-0-1 (ponds) & $\begin{array}{l}\text { Some rabbitbrush, rabbit stgn, } \\
3 \text { small ant mounds }\end{array}$ \\
\hline D & 128-D-1 (burn p1t) & $\begin{array}{l}\text { Tumbleweeds, not sprayed with } \\
\text { herbicide, old garbage, concrete } \\
\text { foundation, pocket mice, deer } \\
\text { tracks }\end{array}$ \\
\hline
\end{tabular}


WHC-EP-0620

Table 5. Insect and Mammal Surveys at 100 Areas Oparable Units. (sheet 2 of 3 )

\begin{tabular}{|c|c|c|}
\hline $\begin{array}{l}\text { Resctor } \\
\text { site }\end{array}$ & Waste site & Comments \\
\hline$H$ & 118-H.1 (burtal ground) & Cobble and lots of tumbleweeds \\
\hline$H$ & 118-H-2 (burtal ground) & Cobble and tumbleweeds \\
\hline$H$ & 118-H-3 (burtal ground) & Cobble and tumbleweeds \\
\hline$H$ & 118-H-4 (burtal ground) & Cobble and tumbleweeds \\
\hline $\mathrm{H}$ & 118-H-5 (burial ground) & Cobble and tumbleweeds \\
\hline$H$ & 116-H-1 (trench) & Cobble and tumblewreds \\
\hline$H$ & 116-H-2 (trench) & Some berm, tumblewneds \\
\hline$\ddot{n}$ & $\begin{array}{l}116-H-7 \\
\text { (rotention basin) }\end{array}$ & $\begin{array}{l}\text { Chain-link fence, cobble, and } \\
\text { tumbleweeds }\end{array}$ \\
\hline H & 126-H-1 (ash pit). & $\begin{array}{l}\text { Lots of small tumbleweeds, lots of } \\
\text { coal slag, some pocket mice, few } \\
\text { ants on perimeter }\end{array}$ \\
\hline$H$ & 128-H-1 (burning pit) & $\begin{array}{l}\text { Lots of small tumbleweeds, } \\
\text { rabbltbrush, deer tracks, pocket } \\
\text { mice }\end{array}$ \\
\hline H & $1607-H-2$ (ttle fleld) & $\begin{array}{l}\text { Cheatgrass, pepper grass, geese } \\
\text { foraging area, one old badger hole }\end{array}$ \\
\hline$k$ & $118-K-1$ (burlal ground) & $\begin{array}{l}\text { Heavily cobbled, no vegetation } \\
\text { present, some burrowing activity } \\
\text { on perimeter }\end{array}$ \\
\hline$k$ & $116-k-1(C r \mid b)$ & $\begin{array}{l}\text { Cobbled, some tumbleweeds, } \\
\text { burrowing activity on perimeter }\end{array}$ \\
\hline$K$ & Inert Waste Landfill & $\begin{array}{l}\text { Lots of cheatgrass and small } \\
\text { tumbleweeds, some debris, some } \\
\text { burrowing activity }\end{array}$ \\
\hline$k$ & 116-K-2 (Trench) & Cobbled, no vegetation \\
\hline$N$ & $1312-N-L E R F$ & $\begin{array}{l}\text { Standing water at times in rubber } \\
\text { bladder, some rabbltbrush, russtan } \\
\text { thistle and cheatgrass on edges }\end{array}$ \\
\hline$N$ & $116-N-3(C r \mid b)$ & $\begin{array}{l}\text { Covered with concrete and cobbled, } \\
\text { Burrowing activity on perimeter }\end{array}$ \\
\hline$N$ & $116-N-2$ (Tank) & $\begin{array}{l}\text { Not cobbled, lots of rabbltbrush, } \\
\text { russian thistle and cheatgrass, } \\
\text { ant and burrowing activity }\end{array}$ \\
\hline
\end{tabular}


Table 5. Insect and Mammal Surveys at 100 Areas Operable Units. (sheet 3 of 3 )

\begin{tabular}{|c|c|c|}
\hline $\begin{array}{l}\text { Reactor } \\
\text { site }\end{array}$ & Waste site & Comments \\
\hline $\mathbf{N}$ & $116-N-1(C r+b)$ & $\begin{array}{l}\text { Dominated by rabbltbrush, Russian } \\
\text { thistle and cheatgrass, varying } \\
\text { topography, ants and burrows } \\
\text { present }\end{array}$ \\
\hline $\mathbf{F}$ & $126-F-1$ (Ash P(t) & $\begin{array}{l}\text { Not cobbled, lots of tumble } \\
\text { mustard russtan thistle and } \\
\text { cheatgrass, some rabbltbrush, } \\
\text { berms present, ant and burrowing } \\
\text { activity }\end{array}$ \\
\hline$F$ & $\begin{array}{l}116-F-14 \text { (rotention } \\
\text { basin) }\end{array}$ & $\begin{array}{l}\text { Cobbled, no vegetation, some ant } \\
\text { activity on perimeter }\end{array}$ \\
\hline $\mathbf{F}$ & 116-F-1 (Trench) & $\begin{array}{l}\text { Cobbled, no vegetation, some ant } \\
\text { activity on perimeter }\end{array}$ \\
\hline $\mathbf{F}$ & 116-F-2 (Tranch) & Cobbled, very little vegetation \\
\hline $\mathbf{F}$ & & $\begin{array}{l}\text { Surface Contamination site, some } \\
\text { grasses, cryptogams, some } \\
\text { burrowing activity }\end{array}$ \\
\hline$F$ & 116-F-9 (Trench) & Cobbled, no vegotation \\
\hline$F$ & 118-F-5 (Burtal Ground) & Cobbled, no vegetation \\
\hline $\mathbf{F}$ & $\begin{array}{l}\text { Surface contamination } \\
\text { Area }\end{array}$ & $\begin{array}{l}\text { PNL Rad Waste Burfal Site, lots of } \\
\text { tumble mustard, cheatgrass, and } \\
\text { Russian thistle, some ant and } \\
\text { burrowing activity }\end{array}$ \\
\hline $\mathbf{F}$ & 118-F-2 (Burtal Ground) & $\begin{array}{l}\text { Cobbled, some ant and burrowing } \\
\text { activity on perimeter }\end{array}$ \\
\hline$F$ & 118-F-4 (Burtal Ground) & $\begin{array}{l}\text { Cobbled, some tumbleweeds and } \\
\text { cheatgrass }\end{array}$ \\
\hline $\boldsymbol{F}$ & $\begin{array}{l}\text { l18-F-1, F-6 (Burial } \\
\text { Ground) }\end{array}$ & Cobbled, no vegetation \\
\hline
\end{tabular}


vegetation sampling (see Tables $\mathrm{H}-10-\mathrm{H}-17$ in Appendix $\mathrm{H}$ ) Indicate extremely low levels of contaminants in vegetation from within the reactor areas, and burrow soll results (Tables G-1 - G-6 in Appendix G) show no evidence of small mammals or ants excavating contaminants from waste stes, which could then have been a pathway for several of the bird feeding groups. The results of raptor pellets and coyote scat sampling (Tables E... - E-2 in Appendix E) show IImited pathway movement of contaminants in the 100 Areas. Previously. however, small mammals have been shown to bring contamination to the surface of waste sites, and rock doves, closely assoclated with bulldings in the 100 and 200 Areas, have been shown to contain contamination (Conkiln ot al. 1982), (Conkiln ot al. 1983).

Mammals (deer, pocket mice, rabbits) also may be in pathways from eating - ither contaminated vegetation or contaminated prey, such as birds or mice. Woodruff at al. (1993) and the sampling reported in this document (coyote scat and mammal burrows) Indicate only localized and low contamination in mammals. such as rabbits from the 100-N Ares (with $88 \mathrm{pCl} / 9$ strontlum-90 in bones, Woodruff ot 11. 1992).

The aquatic birds are alen not expected to be significant pathways. Yoarly aquatle blote sampling (e.g., Woodruff ot al. 1992) and Cushing (1993) indicate slight to undetectable contamination in the 100 Areas prey of many of the aquatic birds listed: periphyton, caddisfly larvie, clams, bass, whiteflish, and salmon. Extensive vegetation sampling results reported in this document also show only rare and low "hits" of contamination in riparian trees, asparagus, and reed canarygrass.

\subsection{PLANT Comunity AMALYSES}

Plant communitles near the Columbla River within the 100 Areas aggregate untts were surveyed during 1991 and 1992. The surveys conststed of searches for rare and endangered plant spectes, qualitative community delinactions, the compllation of spectes lists within the different communlty types, and the Identification and mapping of plants on or near the river shore that have a potential for direct food-chain link to humans or to higher trophic levels within the ecosystem. Appendix I provides a list of all plant specles observed at all the operable units.

\subsection{PLANT COMmunitY DELIMEATIONS}

The plant communities within the 100 Areas operable units immedlately adjacent to the Columbla River have been broadly described as riparian and as - cheatgrass community in areas away from the shoreline (Rogers and Rickard 1977). This classiffcation is broadly correct, but finer delineations are possible. The community delineations described in the following paragraphs were made by field reconnalssance and are strictly qualitative in nature. The delineations were made by visually determining the dominant plant species or vegetation types and were based on the specles most apparent at the time of inspection. In most cases, a particular area was visited at least twice (at different parts of the growing season). For the purposes of this 
repore, the communtey dellineations or descriptions are provided to suggest dominant spectes or associations of spectes that occur within different phystognomic, edaphic, or topographtc units.

Quantitative masurements of spectes frequency, abundance, or coverage may result in silghtly different classifications. Most of the surveys described in the following sectlons are concerned with the thin ripartan strip of vegetation and cover the region from the shoreline of the Columbia River to approximately $400 \mathrm{~m}$ inland. The community changes that can occur over the relatively narrow ripartan zone of the Columbla River are described in Ficketsen of a1. (1980) and Brandt et al. (1993). Beyond this distance from the shore, much of the rest of the area within the 100 Areas operabie untes consists of old agricultural flelds dominated by choatgrass and tumbiemustard, with scattered abandoned orchards and a fow remnant pockets of big sagebrush and gray rabbltbrush.

The shoreline commenteles within the 100 Areas operable units were divided into sections to ease the description process; a map of these sections 1s provided in Appendix I. These divisions are not meant to represent separate, distinct communitles; each diviston comprises several distince vegetation assoclations. Species lists for these areas are provided in Appendix 1. While an attempt was made to Identify all of the specles located in each irea, som were missed undoubtediy because of very low populations, or because the apectes were not in an Identifiable state at the time of the surveys. All spectes Identifications wre made following Hitcheock and Chronquist (1973).

The vegetation within most of the exclusion areas around the reactors and on the cribs and burlal grounds is very sparse or non-existent. Those plant spectes that are presont ars primartly woody plants such as Russian thlstle, Jim HIll mustard, cheatgrass, few essorted herbs and some gray rabbltbrush. Plants such as Russtan thistio and Rabbltbrush are deep rooted and have the potentlal to uptake radionuclides and other contaminants and, therefore, could be part of a pathway of contaminants to other parts of the ecosystem. Some of the radiological control 20nes, such as those near 100-K and 100-N Areas conslst of essentially native vegetation and many of the plant spectas present could contribute to contaninant transport throughout the ecosystem.

\section{1 .1 100-ec Area}

The region upstrean from the 100-BC Area is dominated by a thick stand of wlllow, with interspersed patches of reed canarygrass, sedges, thickspike wheatgrass, and goldenrod. Much of the area is classifled as wotland. The wotland ares is hom to at least three state sensitive spectes (Limosella ecaulis, Lindernia anagallidee, and Cyperus rivularls). Downstream from the 100-BC Area is a cobble shoreline with relatively sparse vegetation. Many white mulberries, ims, and Junipers are present, with an understory of scattered tumblemustard and cheatgrass.

The plant community bounded by the 100-BC Area fence almost entirely comprises the alien species of tumblemustard, Russian thistle, and cheatgrass. Modest stands of gray rabbltbrush are present as well as few scattered bunchgrasses (mostly sand dropseed). 
The section extending from the 100-BC Area to the Allard Pumphouse is primarliy typlfied by relatively steep slopes extending from the dry, cheatgrass-dominated uplands to the river shore, with a falrly narrow ripartan zone. The shoreline itself is steep, with many large cobbles and boulders. The vegetation is primarily reed canarygrass, Poe, sedges, and tickseed.

\section{$0.1 .2100-k$ Area}

The section of shoreline between the Allard Pumphouse and 100-K Area is one of the most diverse vegetative communteles in the 100 Areas. There are many trees in this area, primarliy Mulberries, Elms, and Willows, with nearly 100 other spectes present. Physically, the area is defined by a peninsula that Juts upriver at Coyote Rapids. This forms a backwater area that functions as an isolated pond during times of low water. The standing-watersaturated ground, boulder-strewn peninsula, and the typical sandy/silty ripartan shores provide a vast array of different substrates and habltat types within a relatively small geographic area.

The area bounded by the 100-K Area fence, 11ke many of the other reactor areas, is essentially devold of plant life except for scattered cheatgrass, Russian thistle, and an occastonal rabblebrush.

The strotch of river shore between 100-K and 100-N Areas is characterized by a gently sloping shoreline with a relatively broad riparian zone. The trees (primarlly ofm and mulberry) are distributed primarlly in isolated clumps of five or six individuals. The vegetative community is diverse, showing several distinct vegetative 20nes. Near the water ine, the community is strongly dominated by reed canarygrass, beyond this is a poa pratensis zone, an Agropyron dasytachyum zone, and finally the dryland cheatgrass/ Sandberg's bluegrass community. Each of the vegetation zones has a large number of assoctated spectes, complete listing of the specles observed within the riparian zone between 100-K and 100-N is provided in Appendix 1 . The shoreline itself consists of areas of large boulders and areas of gentiy sloping mudfiats where healthy populations of limosella acaulis can be found.

Beyond the ripartan zone between 100-K and 100-N is a dryland, cheatgrass-dominated community that perhaps typifles much of the 100 Areas. The area has been disturbed in the past, probably by fire, and is now primarlly cheatgrass with approximately 25 assoclated native and introduced spocios.

\section{1 .3 100-N Aros}

Because the 100-N Area is still relatively active, there is very little vegetation present within the 100-N Area proper. To the south and east of the 100-N Area is the approximately 600-acre Knob-and-Kettle topography (Rlckard ot al. 1974) or glant ripple marks (Reidel ot al. 1992). Most of this area has been burned and is now almost completely dominated by cheatgrass and Sandberg's bluegrass.

The shoreline between 100-N and 100-D Areas is extremely steep with a narrow ripartan community clinging to the bottom of the slope. The community is primarily dominated by reed canarygrass. 
WHC-EP-0620

\subsubsection{0-0 Area}

Like the 100-BC, $-K$ and $-N$ Areas, the sparse community bounded by the 100-D Area fences is almost entirely composed of cheatgrass, Russian thistle, and tumblemustard. Few native species are present in this highly disturbed community.

The most obvious feature of the shoreline immediately adjacent to the 100-D Area is a large stand of mature elm trees consisting of approximately 100 individuals. This stand occurs on a sand/cobble bench above the normal high-water mark. There is little or no understory component beneath much of this stand. Surrounding the stand of trees is a short-statured dryland community that includes a number of spectes but is dominated primarlly by cheatgrass, sand dropseed, and tumblemustard. At least 40 species are present on this shelf, including white mulberry, reed canarygrass, and 2 specles of lupine. Downstream from the elm-dominated bench is a riparian communtty dominated by reed canarygrass and bentgrass with a number of white mulberries.

East of 100-0 Area is a sandy, open community with a dominate shrub component of sagebrush and rabbitbrush. The understory consists of the native perennial bunchgrasses, indian ricegrass, squirreltall bottlebrush and needleand-thread grass, along with two spectes of milkvetch and prickly-pear cactus.

\subsubsection{Ripartan Communtties in the 100-HR-3 Operable Unit}

At the northeast corner of 100-D Area is a mixed community dominated in sections by big sagebrush with an understory of cheatgrass and tumblemustard. There are two abandoned apricot orchards and a considerable amount of oldfield vegetation in this area. Other readily apparent species include Munro's globemallow, yellow bee-plant, and gray rabbitbrush. The majority of the dryland areas within the 100-HR-3 operable unit are abandoned agricultural flelds with a few abandoned orchards. There are very few isolated pockets of native vegetation, cheatgrass is the most abundant species, with various alien annuals, globemallows and, along roadway edges, sand dropseed and needie-andthread grass.

The shoreline to the northeast of 100-0 Area transitions from the higher ground of the reactor area through a low-lying zone of undulating topography, to a broad, flat, rocky plain. Several community types can be discerned over this topographic gradient.

The region in the vicinity of river kilometer 605.8 , about $1 \mathrm{~km}$ north of 100-D Area, is characterized by undulating topography and coarse sands. Many of the topographic depressions become saturated for varying pertods of time. These swales are dominated by sedges, with smaller populations of rushes and clover-fern. Between the swales, the community is dominated by thickspike wheatgrass, bluegrass, and Russlan thistle. A distinct community dominated by wooly mullein and cocklebur is at the northern edge of this zone. The shoreline itself is heavily dominated by reed canarygrass. The area around river mile 376.25 is characterized by a relatively flat, cobble plain, with a large sand hill, approximately $3 \mathrm{~m}$ tall and $100 \mathrm{~m}$ long, located about $500 \mathrm{~m}$ from the shoreline. The sandhill itself is dominated by wheatgrass, with several individuals of giant wildrye. East of the sandhill the community is dominated by Russian thistle, cheatgrass, tumblemustard, with ifttle else 
present. The plain to the west of the sandhill is characterized by pockets of sedges, wheatgrass, cheatgrass, and tumblemustard. The Columbia tickseed is evident during the summer. The plain appears to be part of an old riverbed and appears to be moist if not inundated at times of peak river flow.

The river bends sharply to the east at approximately river mile 375.75 , with a backwater inlet formed after the river bends. This creates, at least during high water, a stubby peninsula that points north at the bend in the river (see Figlire I-1, Appendix I). Several distinct communities can be delineated on this peninsula. On the western half is an area consisting almost exclusively of diffuse knapweed. Downriver from the knapweed community the surface is dominated by wheatgrass, and off the southern end of the inlet is an area dominated by red three-awn. An area between all three of these communities is dominated by cheatgrass and Russian thistle. Several sand mounds are located on the east side of the wheatgrass community at the tip of the peninsula. These mounds are dominated by giant wildrye and slimleaf goosefoot, with a lesser component of lupine (two different species). The shoreline is dominated by reed canarygrass and bluegrass, with several small, isolated elms and white mulberries.

Continuing west from the peninsula, the shoreline gradually changes from a gentle rocky plain, with a broad riparian habitat, to a steep, distinct drop-off with a narrow riparian zone. The soil substrate gradually changes from large cobbles at the south end of the backwash to gravelly saind farther east. The edges of the backwash area are dominated by wetland species and the inlet usually has standing water unt 11 midsummer. A patch of inundated willows dominates the mouth of the backwash, and little vegetation is present in the rest of the area having standing water. The dryland communities to the east side of the backwash are dominated by cheatgrass with subdominant components of sand dropseed at the western end with Columbia daggerpod and Gray's desertparsley becoming common farther to the east.

The shoreline is a wetter, periodically saturated area with large components of horsetail, sedges, bluegrass, and yellow sweet clover. This river stretch culminates with two stands of mature trees (primarily black locust) between river miles 373.75 and 373.0 . The community between and underneath the stands of crees is best described as weedy, primarily cheatgrass, flixweed, and tumblemustard. A few giant wildrye individuals are present between the stands of trees. The shoreline in this area is steep but relatively sandy and is dominated by reed canarygrass and bluegrass, with numerous white mulberries and several golden currant bushes.

\section{$6.1 .6100-H$ Area}

The shoreline adjacent to $100-\mathrm{H}$ area is steeply sloped, with a narrow riparian zone dominated by reed canarygrass and bluegrass, and several white mulberries and golden currants. The shoreline flattens out to the south of $100-\mathrm{H}$ Area in the vicinity of $\mathrm{H}-\mathrm{slough}$.

There are no fences remaining around the 100-H Area, but most of the area that was included in the exclusion zone is highly disturbed, with many burial grounds, cribs, and old building sites visible. Much of the area is dominated by gray rabbitbrush and cheatgrass. The roadways are lined with sand dropseed and Russian thistle. 
WHC -EP-0620

\section{1 .7 100-F Area}

The section of shoreline between the 100-H and 100-F Areas from $\mathrm{H}-\mathrm{s}$ lough through the White Bluffs Townsite was not specifically examined nor characterized for this report because it is outside the boundaries of any of the 100 Areas operable units. However, several distinct communities can be discerned from passive observation. The area referred to as the Whitebluffs or $\mathrm{H}$-slough is a broad wetland formed in the shallows between the main shoreline and an area of high ground that is an island during times of peak riverflow. Above the shoreline in this area is a community dominated by very large sagebrush and giant wildrye. The Whitebluffs Ferry Site is south of the sagebrush/wildrye community. The Ferry site is dominated by a large collection of mature trees, primarily cottonwood and black locust. The understory is very weedy, with much of the ground surface completely covered with Russian knapweed.

The area included within the former boundaries of 100-F Area is primarily dominated by gray rabbitbrush and cheatgrass. Sand dropseed can be found along the roadways. There are also numerous, al though scattered, remnant trees, including sycamores, mulberries, junipers, elms, and poplars.

The shoreline adjacent to the 100-F Area is very steep, with a narrow riparian zone. Much of the shoreline consists of large cobbles and boulders. At the southern end of the boundaries of the 100-F Area the shoreline abruptiy flattens into a rocky plain that eventually graduates into the backwater, wetland area known as F-slough. The rocky plain has conspicuous populations of lupine and Gray's desertparsley.

\subsection{THREATENED AND ENDANGERED PLANT SPECIES}

There are 12 plant species known to be on or near the Hanford Site that are 1 isted by the Washington State Natural Heritage Program (1990) as endangered, threatened, or sensitive (Sackschewsky 1992). These species are listed in Table 6. The two state endangered and the two state threatened species on this list are al so listed as candidates for federal protection under the Endangered Species Act of 1973. Special emphas is was placed on the search for all 12 species while conducting the community delineation and species inventory field work. An assessment of the impacts of characterization activities on threatened, endangered, and sensitive plant species is available in Sackschewsky (1992).

The persistent sepal or Columbia yellowcress is found along the Hanford Reach from the Vernita bridge to the 300 Area (Sauer and Leder 1985). During the FY 1991 and 1992 field surveys, the species was located in the vicinity of 100-B Area, adjacent to the Allard pumphouse (about $3 \mathrm{~km}$ north of 100-D Area) and at the Hanford Townsite. Previously the persistent sepal yellowcress has been found near 100-D Area, White Bluffs, and on many of the Columbia River islands.

The southern mudwort and the false pimpernel also were located in the wet 1 and area just west of the 100-B Area. The southern mudwort al so was found on mudflats east of 100-K Area. Both of these species are likely to be found at other wetland areas along the Hanford Reach of the Columbia River. 
WHC-EP-0620

Table 6. Hanford Site Endangered, Threatened, and Sensitive Plant Species."

\begin{tabular}{|c|c|c|c|}
\hline Scientific name & Common name & Family & $\begin{array}{l}\text { Washington } \\
\text { State status }\end{array}$ \\
\hline $\begin{array}{l}\text { Rorippa columbiaeb Suksd. } \\
\text { ex Howell }\end{array}$ & $\begin{array}{l}\text { persistent sepal } \\
\text { yellowcress }\end{array}$ & Brassicaceae & Endangered \\
\hline $\begin{array}{l}\text { Artemisia campestris L } \\
\text { ssp. borealis (Pall.) } \\
\text { Hali \& Clem. var. } \\
\text { wormskioldif (Eess.) } \\
\text { Cronq. }\end{array}$ & $\begin{array}{l}\text { northern } \\
\text { wormwood }\end{array}$ & Asteraceae & Endangered \\
\hline $\begin{array}{l}\text { Astragalus columbianus } \\
\text { Barneby }\end{array}$ & $\begin{array}{l}\text { Columbia milk- } \\
\text { vetch }\end{array}$ & Fabaceae & Threatened \\
\hline $\begin{array}{l}\text { Lomatium tuberosumb } \\
\text { Hoover }\end{array}$ & $\begin{array}{l}\text { Hoover's desert- } \\
\text { parsley }\end{array}$ & Aplaceae & Threatened \\
\hline $\begin{array}{l}\text { Cryptantha interrupta } \\
\text { (Greene)Pays. }\end{array}$ & $\begin{array}{l}\text { bristly } \\
\text { cryptantha }\end{array}$ & Boraginaceae & Sensitive \\
\hline $\begin{array}{l}\text { Cryptantha leucophaea } \\
\text { Doug1. Pays }\end{array}$ & gray cryptantha & Boraginaceae & Sensitive \\
\hline $\begin{array}{l}\text { Erigeron piperianus } \\
\text { Cronq. }\end{array}$ & Piper's daisy & Asteraceae & Sensitive \\
\hline Carex densa L.H. Bailey & dense sedge & Cyperaceae & Sensitive \\
\hline Cyperus rivularis Kunth & $\begin{array}{l}\text { shining } \\
\text { flat sedge }\end{array}$ & Cyperaceae & Sensitive \\
\hline Oenothera pygmaea & $\begin{array}{l}\text { dwarf evening } \\
\text { primrose }\end{array}$ & Onagraceae & Sensitive \\
\hline $\begin{array}{l}\text { Limosella acaulis Ses.\& } \\
\text { Moc. }\end{array}$ & southern mudwort & Scrophulariaceae & Sensitive \\
\hline $\begin{array}{l}\text { Lindernia anagallidea } \\
\text { (Michx.) Pennel1 }\end{array}$ & false pimpernel & Scrophulariaceae & Sensitive \\
\hline
\end{tabular}

All of these species have been reported on or near the Hanford Site.

Indicates candidates on the 1990 Federal Register, Notice of Review. 
The shining flatsedge and the dense sedge (Carex densa) have been reported from the 100-BC wetland (Washington Natural Herltage Program [WNHP] Database). The shining flatsedge has not been subsequently relocated at that or any other location on the Hanford Site; however, it has been reported from the Priest Rapids area (Mastroglusseppe and G111 1983).

Specimens resembling the dense sedge were collected at the 100-BC wetland and numerous other sites within the 100 Areas during the FY 1992 field surveys. Thase were subsequently identifled as Fox sedge (Carex vulpinoidea) (Appendix K). These two sedge species share several characteristics in common and can easily be confused if mature material is not avallable. Dense sedge is primarlly a California species, with Washington State at the extreme northern periphery of its distribution. The site of the Hanford Site population that was reported to WNHP was resampled during 1992 and was identified as $C$. vulpinoidea; therefore, it is unlikely that $C$. densa inhabits the Hanford Site, espectally because it is otherwise known only from Clark and Wahkiakum counties in southwestern Washington, and $C$. vulpinoidea has been reported previously at Priest Raplds (Mastrogiusseppe and G111 1983). However, unt il the specimen that the original WNHP report was based is relocated and examined, the potential for finding $C$. densa on the Hanford site cannot be ignored.

The gray cryptantha was observed in the dunes area to the east of the 100-D Area. This species is usually found among sand dunes, espectally those south of the Hanford Townsite.

None of the other threatened, endangered, or sensitive plant specles were observed in the 100 Areas during Fy 1991. The bristly cryptantha and the dwarf evening primrose exist in Frankl in County directly across the river from the 300 Area. The dwarf evening primrose is al so known to exist just north of the Wye barricade. Piper's daisy occurs on Umtanum Ridge on the western edge of the Hanford Site and was found during FY 1991 in the vicinity of B-Pond near the 200 East Area. Plper's dalsy has been reported near 100-H reactor (Sackschewsky et al. 1992) but has not subsequently been relocated in that area. The Columbia milkvetch is found on the Yakima Firing Range and has been found on Umtanum Ridge at the western edge of the Hanford Site and at Priest Rapids. The bristly cryptantha, dwarf evening primrose, Piper's daisy, and the Columbla milkvetch could inhabit certain communitles in the 100 Areas. Hoover's desertparsley inhabits steep talus slopes near Priest Rapids Dam at the western edge of the Hanford Site. Hoover's desertparsley is not expected to be found in the 100 Areas.

The northern wormwood is one of the rarest plant taxa in Washington State. Northern wormwood is known from only two populations, both near the Columbia River. One population is near The Dalles, Oregon, and the other is located near Beverly, Washington, approximately $16 \mathrm{~km}$ northeast of the Vernita Bridge. Many plant communities along the Columbia River on the Hanford Site resemble those near Beverly, Washington. Because of the proximity of one of the known populations to the Hanford Site, the similarity of habitat, and the extreme rarity of the taxa, special emphasis has been placed on locating any populations of northern wormwood that may occur on the Hanford Site. Currently, no populations of the northern wormwood (Artemisia campestris wormskioldii) have been found on the Hanford Site; however, another variety of the same species (Artemisia campestris scouleriana) is very common on the 
WHC-EP-0620

Hanford Site and occurs sympatrically with the population of var. wormskioldif near Beverly, Washington. The scouleriana variety is not a special-status taxa.

\subsection{OTHER SPECIES OF INTEREST}

Several plant groups are of interest in the course of these investigations because of the possibilities for contaminant transport to higher trophic levels within the ecosystem and for short or direct pathways of radionuclides to humans. The three plant groups monitored during Fy 1991 were reed canarygrass, asparagus, and trees. The methods and results of direct vegetation sampling for radionuclides are provided in section 7.0 of this report.

If radionuclides are taken up by plants, the radionuclides could eventually reach the human population. This may result from direct consumption of contaminated plants by humans, or indirectly through human consumption of animals that have eaten the contaminated vegetation. Direct pathways to humans are possible through plants such as asparagus and mulberries. Indirect pathways can result from human consumption of deer or rabbits that have consumed contaminated asparagus, from deer that have consumed mulberries, or geese that have consumed reed canarygrass.

Besides asparagus and mulberries, there are well over 100 additional species on the Hanford Site that can be considered edible (Sackschewsky et al. 1992). Table 7 provides a listing of a few of the important edible spectes known to inhabit the 100 Areas of the Hanford site. Some of these plants may not be obvious consumables to all readers, but they have been used as food sources by other cultures. The most desired foods are asparagus and the fruits from the abandoned apple, pear, peach, and apricot orchards along the Columbia River as well as various berries. Most of the other species would only be sought by experienced natural-food enthusiasts. Soldat et al. (1990) have considered wild edible plants in evaluating potential radiation doses to people harvesting plants and wildlife in the 100 Areas.

The basic distributions of trees, asparagus, and reed canarygrass were determined in the 100 Areas. Reed canarygrass is extremely common all along the shore of the Columbla River. At many locations reed canarygrass can be considered the dominant species of shoreline vegetation. Asparagus is al so widely distributed. It is normally represented by widely scattered clumps; it rarely constitutes a dominant component of any community. Abandoned asparagus flelds are st 111 readily discernable at the Hanford townsite.

Trees along the shoreline within the 100 Areas were mapped individually. The most prevalent species is white mulberry, with two major stands of black locust and one major stand of Siberian elms (below 100-D Area). Elms also occur scattered along the shore at many locations. Other species observed include golden currants, apricots, junipers, and wlllows. Upstream from the 100-B Area the most common tree is the willow, with a few scattered elms and white mulberries. Shoreline maps showing the location and identity of all trees in the 100 Areas are archived in field logbook \#WHC-N-534. 
Table 7. Hanford Stte Edible Plants.

\begin{tabular}{|c|c|c|}
\hline Scientific name & Common name & Plant parts used \\
\hline Amaranthus spp. & amaranth, plgweed & Teaves, seeds \\
\hline Balsamorhiza careyane & balsamroot & Whole plant \\
\hline Gallum aparine & cleavers & shoots, seeds \\
\hline Lonat ium spp. & biscuitroot & roots, seods \\
\hline Rubus spp. & blackberry, raspberry & fruits \\
\hline Scirpus spp. & bulrush & $\begin{array}{l}\text { roots, shoots, pollen, } \\
\text { seeds }\end{array}$ \\
\hline Typhe spp. & cattall & pollen, roots \\
\hline Clchorium intybus & chtcory & leaves, roots \\
\hline Prunus spp. & cherries, peaches, etc. & fruit \\
\hline Pyrus spp. & pear, apple & frutt \\
\hline Rosa woodsil & Wood's rose & rosehips, flowers \\
\hline Taraxacum officinale & dandelion & leaves, roots, flowers \\
\hline Rumex SPP. & dock, sorrel & leaves \\
\hline Oenothera spp. & evening primrose & young roots \\
\hline Ribes Spp. & gooseberry, currant & fruit \\
\hline Juniperus scopulorum & Juniper & "berries" \\
\hline Chenopodiun albun & Tamb's quarters & leaves, young stems \\
\hline Calochortus macrocarpus & sagebrush marlposa $111 y$ & bulbs \\
\hline Montia perfollata & miner's lettuce & leaves \\
\hline Mentha spp. & $\operatorname{mint}$ & leaves \\
\hline Alliun Spp. & onton & butbs \\
\hline Leptdiun spp. & pepperweed & fruits, seeds \\
\hline Capsella bursa-pastoris & shepherd's purse & leaves, seeds \\
\hline Plantago spp. & plantain & leaves \\
\hline Opuntia spp. & prickly pear & frutts, stems \\
\hline Portulace oleracea & common purslane & leaves, stems \\
\hline Tragopogon dubius & salsify, goatsbeard & roots \\
\hline Asclepias speciosa & showy milkweed & flowers, shoots \\
\hline Veronica spp. & brooklime & loaves, stems \\
\hline Helianthus annuus & common sunflower & seeds \\
\hline Cirsium spp. & thistle & peeled stems, roots \\
\hline Vicia cracca & bird vetch & fruits \\
\hline Asparagus officinalis & asparagus & young shoots \\
\hline Lactuca serriola & prickly lettuce & young leaves \\
\hline Morus albe & white mulberry & fruit \\
\hline Juglans nigra & black walnut & nuts \\
\hline Salix spp. & willow & bark, leaves \\
\hline $\begin{array}{l}\text { Rorippa nasturtium- } \\
\text { gquatice }\end{array}$ & watercress & Teaves \\
\hline Apocynum sibericum & Indian hemp & bark \\
\hline Achilled millefolium & yarrow & leaves \\
\hline
\end{tabular}

spp. - species, more than one. 
WHC-EP-0620

Blota and soll samples were collected at several sites associated with the operable units. Media that was collected included asparagus, reed canarygrass, water milfoll, tree leaves and limbs, raptor pollets, coyote scat, and soll samples from ant mounds and small mammal burrows. Samples were collected following the methodologles given in the Description of Work for this project (Landeen 1992). All samples were sent to an offs te laboratory (TMA-NORCAL) for radionuclide and inorganic constituents analys is. Maps showing sample locations of all media collected are presented in Appendix A. Detalls of these sampling efforts are given in the following sections.

\subsection{Asparacus}

Asparagus (Asparagus officinalis) was collected during Aprll 1991 and May 1992 at the sites depicted in Figure A-1 of Appendix A. A dupl icate sample was collected during both collecting periods. Control samples were collected above Vernita Bridge and at Horn Raplds on the Yakima River. The samples were analyzed for total gamma radiation, strontlum-90, technetfum-99, and total TAL metals.

\subsection{REED CANARYGRASS}

Reed canarygrass (Phalaris arundinacea) was collected during July 1991 and 1992 at the sites depicted in Figure A-2 of Appendix A. Three to six samples of reed canarygrass were collected at each sampling location. Duplicate samples were collected both years and control samples were collected upriver of the Vernita Bridge next to the Columbia River. All of the samples were analyzed for the same constituents as the asparagus.

\subsection{TREE LEAVES NDO LIMBS}

Mulberry (Morus alba) leaves and limbs were collected during July 1991 and July and October 1992 at the sites indicated in Figure A-3 of Appendix A. Two samples of tree leaves were collected at every sampling location. Duplicate samples were collected both years and control samples were collected above Vernita Bridge next to the Columbla River. All of the samples were analyzed for the same constituents as the asparagus. Some of the tree leaves were also analyzed for tritium.

\subsection{MATER MILFOIL}

At the request of the U.S. Environmental Protection Agency, three water milfoll (Myriophyllum spicatum) samples were collected at $N$ Springs on October 7, 1992, and one control sample was collected above Vernita Bridge in the Columbia River (Figure A-3). The samples were analyzed for TAL metals, strontium-90, and total gamma radiation. 


\subsection{ANIMALS (COYOTES ANO RAPTORS)}

Raptor pellets and coyote scat were collected near some of the reactor areas in the 100 Areas (Flgure A-4). Raptors are nenerally defined as birds of prey, which include hawks, eagles, and owls. Control samples were collected on the north side of the Columbla River on the Wahluke slope. The samples were analyzed for TAL metals, strontlum-90, and total gamma radiation.

\subsection{ANT MOUNDS NMO SHALL MAMHL BURROWS}

Soll samples from active harvester ant (Pogonomyrmex owyheef) mounds and small mammal burrows were collected adjacent to waste sites at each of the reactors in the 100 Areas (Table 8). Harvester ants form their mounds by excavating and bringing soll and rock particles to the surface. Harvester ants sometimes excavate materials from as far as $5 \mathrm{~m}$ down (Porter and Jorgensen 1988) and, thus, can serve as indicators of environmental quality. Previous work in the 300 Area (Fitzner et al. 1979) has shown the abundance of harvester ants on disturbed soli such as wastes sites and their potential for deep excavation of contaminants.

Small mammals also form mounds from excavated materlal brought to the surface and serve as indicators of contaminant uptake (Landeen and Mitchell 1981 and 1982). Burrows sampled were made by the Great Bas in pocket mouse (Perognathus parvus), which is the most abundant small mammal at the Hanford site. Pocket mice are prolific burrowers and excavate burrow systems usually 0.6 to $1.2 \mathrm{~m}$ in depth, but might burrow deeper (Landeen and Mitchell 1981).

The samples were sent to an offsite laboratory and analyzed for TAL metals, stront tum-90, and cestum-137.

\subsection{DATA AMALYSIS}

Results of all the analytical data collected are presented in Appendixes $B$ through $H$. Information regarding sample identification numbers, sample locations, and assoclated data validation qualiffers are presented also. The data presented below is compared with offsite control samples collected as part of this study and other similar efforts (Table 9). The data are also compared with the average values from all media collected as part of this study (Table 10) as well as other sampling efforts prevlously conducted at the Hanford site. These previous sampling efforts include sediment sampling assoctated with springs adjacent to the Columbla River throughout the 100 Areas (Table 11) and the 2101-M Pond characterization study on the 200 Area plateau (DOE-RL 1993) (Table 12). Data are also compared with soll threshold values, which were calculated in Ritzville silt loam at the Hanford Site (Table 13) (Wildung et a1. 1986) and other soll threshold values and maximum values reported in DOE-RL (1993) from the Hanford Stte (Table 14). The soll threshold values presented in Tables 13 and 14 indicate differences 
Table 8. Ant Mound and Burrow Sampling Locations.

\begin{tabular}{|c|c|c|c|c|}
\hline Aesetor & inple & inple madle & Waste ste 10 & Deseription \\
\hline 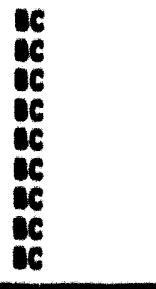 & 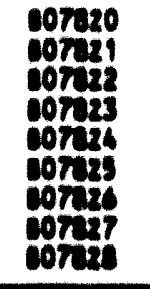 & $\begin{array}{l}\text { mi } \\
\text { burrow } \\
\text { burrow } \\
\text { burrow } \\
\text { ent } \\
\text { ent } \\
\text { ent } \\
\text { ont } \\
\text { burrow }\end{array}$ & 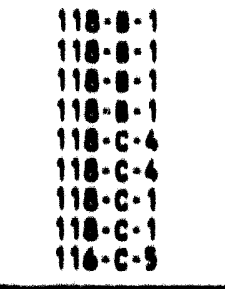 & 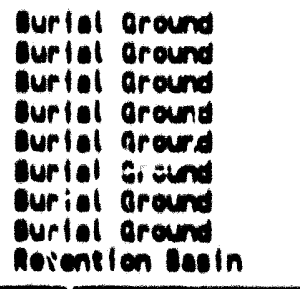 \\
\hline 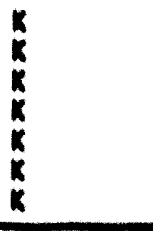 & 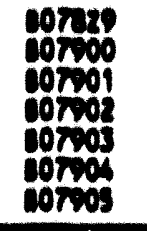 & $\begin{array}{l}\text { burrow } \\
\text { burrow } \\
\text { burrow } \\
\text { burrow } \\
\text { burrow } \\
\text { burrow } \\
\text { burrew }\end{array}$ & $\begin{array}{l}110 \cdot k \cdot 1 \\
118 \cdot k \cdot 1 \\
118 \cdot k \cdot 1 \\
116 \cdot k-1 \\
116 \cdot k \cdot 1 \\
110 \cdot k \cdot 1 \\
116 \cdot k \cdot 1\end{array}$ & $\begin{array}{l}\text { ourlal oround } \\
\text { due ial oround } \\
\text { curlal oround } \\
\text { crib } \\
\text { crib } \\
\text { crib } \\
\text { crib }\end{array}$ \\
\hline $\begin{array}{l}n \\
n \\
n \\
n \\
n \\
n \\
n\end{array}$ & & $\begin{array}{l}\text { burrow } \\
\text { burrow } \\
\text { burrow } \\
\text { ont } \\
\text { ont } \\
\text { ene } \\
\text { ent } \\
\text { burrow }\end{array}$ & $\begin{array}{l}116 \cdot n \cdot 2 \\
116 \cdot n \cdot 3 \\
116 \cdot n \cdot 1 \\
116 \cdot n \cdot 1 \\
116 \cdot n \cdot 1 \\
116 \cdot n-1 \\
116 \cdot n-1 \\
116 \cdot n \cdot 1\end{array}$ & $\begin{array}{l}\text { etorese tenk } \\
\text { erib } \\
\text { erib } \\
\text { erib } \\
\text { erib } \\
\text { erib } \\
\text { erib } \\
\text { erib }\end{array}$ \\
\hline $\begin{array}{l}0 \\
0 \\
0 \\
0 \\
0 \\
0 \\
0 \\
0\end{array}$ & & $\begin{array}{l}\text { ont } \\
\text { burrew } \\
\text { ent } \\
\text { burrow } \\
\text { cont } \\
\text { burrow } \\
\text { cut } \\
\text { burrew }\end{array}$ & $\begin{array}{l}116 \cdot 0 n \cdot 9 \\
116 \cdot 04 \cdot 9 \\
116-01 \cdot 9 \\
116 \cdot 0 \cdot 3 \\
116 \cdot 0 \cdot 6 \\
116 \cdot 0 \cdot 1 \\
116 \cdot 0 \cdot 2 \\
116 \cdot 0 \cdot 2\end{array}$ & 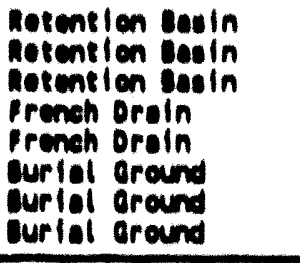 \\
\hline 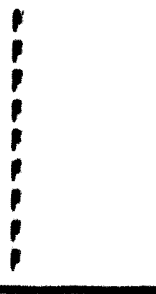 & & $\begin{array}{l}\text { ent } \\
\text { burrow } \\
\text { burrow } \\
\text { ent } \\
\text { burrow } \\
\text { ent } \\
\text { burrow } \\
\text { ent } \\
\text { burrow }\end{array}$ & $\begin{array}{l}126 \cdot 1 \cdot 1 \\
126 \cdot 1 \cdot 1 \\
126 \cdot 1 \cdot 1 \\
128 \cdot 1 \cdot 1 \\
116 \cdot 1 \cdot 16 \\
116 \cdot 1 \cdot 1 \\
116 \cdot 1 \cdot 2 \\
116 \cdot 1 \cdot 2\end{array}$ & 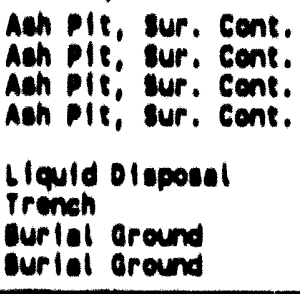 \\
\hline $\begin{array}{l}n \\
n \\
n \\
n \\
n \\
n \\
n \\
n\end{array}$ & & $\begin{array}{l}\text { ent } \\
\text { ent } \\
\text { burrow } \\
\text { burrow } \\
\text { ent } \\
\text { ent } \\
\text { burrow } \\
\text { ent }\end{array}$ & $\begin{array}{l}118 \cdot n \cdot 3 \\
118 \cdot n \cdot 3 \\
118 \cdot n \cdot 3 \\
118 \cdot n \cdot 3 \\
116 \cdot n \cdot 7 \\
116 \cdot n \cdot 7 \\
116 \cdot n \cdot 7 \\
118 \cdot n \cdot 2\end{array}$ & 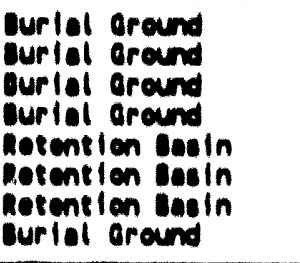 \\
\hline
\end{tabular}


Table 9. Average Values of all Control Samples Collected in 1991 and 1992 as Part of 100 Araas

Ecological Investigations and Other studies. (sheet 1 of 2)

\begin{tabular}{|c|c|c|c|c|c|}
\hline \multirow{2}{*}{ Constituent } & \multicolumn{5}{|c|}{ enple medie and totel numer of semples } \\
\hline & mien & covote & $\begin{array}{c}1 \\
\text { mitholt }\end{array}$ & $2101 \cdot 110011$ & thine \\
\hline Alualnen & 1810.00 & 3005.00 & 967.00 & $m m .00$ & 1097.67 \\
\hline antiency & 3.38 & 3.43 & 19.60 & $\cdots$ & 3.33 \\
\hline Arsente & 3.13 & 2.93 & 3.60 & $\because$ & 1.20 \\
\hline carlen & 69.00 & 01.05 & 116.00 & ex.25 & 123.90 \\
\hline cervillive & 0.16 & 0.00 & 0.03 & 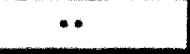 & 0.20 \\
\hline coniv & 0.21 & 0.20 & 3.50 & 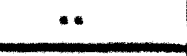 & 0.01 \\
\hline calelu & 40.4.67 & 57580.00 & 20200.00 & 4300.00 & Sick. 67 \\
\hline chrealu & 6.27 & 4.60 & 7.00 & 0.30 & 6.37 \\
\hline cemalt & 1.70 & 3.00 & 3.10 & 0,24 & 1.68 \\
\hline cerpor & 17.37 & 15.03 & 20.00 & 11.7 & 22.10 \\
\hline Iren & 6016.67 & 6700.00 & 1700.00 & 2840.00 & 1503.67 \\
\hline Lead & 2.0 & 3.18 & 9.80 & 6.25 & $3 . n$ \\
\hline menestiv & 346.67 & $\$ 010.00$ & 2650.00 & 439.00 & 19176.67 \\
\hline neromenese & 0.13 & 131.90 & 370.00 & 397.90 & 09.10 \\
\hline mereury & 0.00 & 0.00 & 0.67 & $\ddot{*}$ & 0.0 \\
\hline Wlekel & 3.17 & 4.19 & 9.00 & 0.50 & 3.23 \\
\hline Dotcoselun & 1427.00 & 201.7 & 2570.00 & 1490.00 & 61000.00 \\
\hline coleniven & 3.03 & 3.63 & 4.60 & 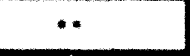 & 3.63 \\
\hline olliver & 0.92 & 0.51 & 2.00 & $\ddot{m}$ & 1.7 \\
\hline cadin & 1524.67 & 2319.00 & 1190.00 & 109.00 & 009.00 \\
\hline Thallin & 0.48 & 0.26 & 6.30 & $\ddot{\cdots}$ & 1.07 \\
\hline Venedlum & 7.23 & 12.93 & 5.40 & 0.25 & 3.21 \\
\hline $2 \ln s$ & 216.07 & 161.60 & 206.00 & 42.7 & 126.13 \\
\hline orenich & $m$ & $m$ & m & M & $m$ \\
\hline
\end{tabular}

worts All wotele ore reparted in m/ke.

- Analve found In eoseclated blank as mall es semple.

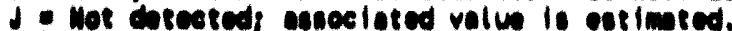

in - Menresorteble.

- Dote ore unueble.

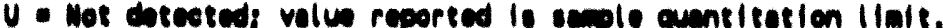
|lalt.

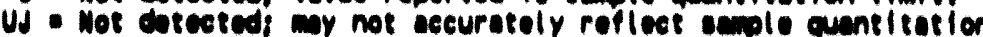


Table 9. Average Values of all Control Samples Collected in 1981 and 1992 as Part of 100 Areas Ecological Investigations and Other Studies. (sheot 2 of 2)

\begin{tabular}{|c|c|c|c|c|}
\hline Cencellecunt & 1 & exiss & acprimave & atre \\
\hline Alvinen & 200.60 & 707.67 & 20.09 & 4840.00 \\
\hline melemy & 3.80 & 3.49 & 7.01 & 3.10 \\
\hline Arsenle & 3.00 & 1.18 & 1.39 & 2.30 \\
\hline Cerlu & 108.09 & 26.20 & 3.00 & 0.30 \\
\hline envilliv & 0.13 & 0.10 & 0.01 & 0.13 \\
\hline entan & 0.10 & 0.47 & 0.00 & 0.11 \\
\hline entele & 11700.00 & 394.17 & 2028.90 & 0100.00 \\
\hline chrolo & 10.60 & 2.00 & 3.20 & 5.50 \\
\hline entols & 10.60 & 0.60 & 1.40 & 10.10 \\
\hline Cecripor & 11.60 & 0.45 & 0.80 & 12.60 \\
\hline Iren & 0000.00 & 135.7 & 33.50 & 1000.00 \\
\hline lead & 13.50 & 2.06 & 0.07 & 5.90 \\
\hline nenesto & 910.00 & 2146.67 & 1137.90 & 1020.00 \\
\hline nnes & 319.00 & $r .13$ & 12.5 & 260.00 \\
\hline mreury & 0.08 & 0.17 & 0.34 & 0.05 \\
\hline NIehal & 0.70 & 2.72 & 3.31 & 0.90 \\
\hline Potcestiv & 1070.00 & 16620.33 & $26 \pi n .00$ & 60.00 \\
\hline coleniu & $0 . \pi$ & 1.7 & 3.27 & 0.0 \\
\hline sllwer & 0.07 & 1.01 & 1.03 & 0.01 \\
\hline cediv & 201.00 & 17.49 & 171.90 & 101.00 \\
\hline Thalliv & 0.7 & 0.46 & 0.91 & 0.6 \\
\hline venedion & 92.10 & 2.60 & 1.33 & 92.20 \\
\hline $81 \mathrm{~m}$ & 113.00 & 70.43 & 50.20 & 43.30 \\
\hline Grenla & in & min & in & in \\
\hline
\end{tabular}

motel All motels ore reperted in ma/kg.

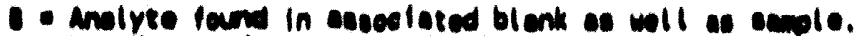

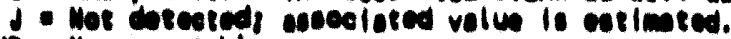

in - Menresertcble.

- Dete are vinable.

llalt.

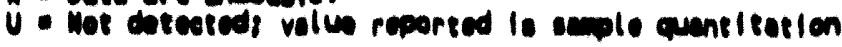
quentitotion IInle. 
Table 10. Average Values of all Samples Collected in 1991 and 1992 is Part of 100 Areas Ecological Investigations and Other Studies. (sheet 1 of 2)

\begin{tabular}{|c|c|c|c|c|c|c|}
\hline \multirow{3}{*}{ cometitument } & \multicolumn{6}{|c|}{ 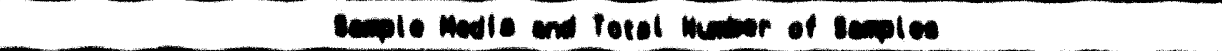 } \\
\hline & 4 & 7 & 3 & 20 & 80 & 27 \\
\hline & neren & covere & MItrot! & Eolmurs & $2101 \cdot 11011$ & inutes \\
\hline Alving & 40.6 & 1804.00 & 493.33 & 081.00 & $8 \times 0.00$ & 24.70 \\
\hline mining & 3.6 & 3.98 & 0.10 & 9.31 & 0.00 & 0.04 \\
\hline Arecule & 3.35 & 3.6 & 4.40 & $\cdots$ & 0.23 & 1.48 \\
\hline onton & 93.10 & 49.30 & 124.31 & 60.60 & 7.90 & 113.60 \\
\hline corvillim & 0.18 & 0.17 & 0.30 & 0.31 & 0.20 & 0.8 \\
\hline sechiv & 0.13 & 1.04 & 4.90 & 0.5 & 0.30 & 0.6 \\
\hline colelun & 11540.00 & 4ist9.16 & 21100.00 & 3097.30 & 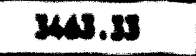 & $m 618.12$ \\
\hline enrealu & 3.70 & 6.6 & 0.9 & 35.15 & 10.38 & 2.7 \\
\hline cecols & 0.01 & 1.60 & 3.43 & 7.44 & $9 . \pi$ & 1.52 \\
\hline Comer & 13.4 & 15.34 & 40.97 & 19.03 & 910.20 & 28.33 \\
\hline Iren & 2167.90 & 394,25 & I8n. 17 & 3200 . 70 & 20924.07 & 400.70 \\
\hline lew & 5.10 & 7.0 & 19.20 & $\cdots$ & 0.60 & 1.00 \\
\hline menevive & 203.50 & 4431.43 & 4383.33 & $3 \times 10$ & 373.00 & 13112.6 \\
\hline nuncroses & 157.6 & 125.00 & 707.67 & 316.00 & 271.00 & 111.4 \\
\hline mereury & 0.00 & 0.00 & 0.8 & 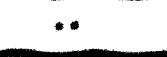 & 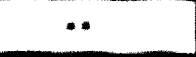 & 1.00 \\
\hline Mlekel & 1.6 & 2.14 & 10.03 & 12.40 & 0.70 & 3.62 \\
\hline Potendiv & 1427.90 & 394.20 & 490.33 & 000.30 & 1095.7 & $\cos 2.50$ \\
\hline solenive & 4.11 & 3.4 & 1.97 & 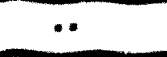 & 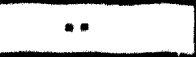 & 3.31 \\
\hline sllver & 0.34 & 0.52 & 0.6 & 1.18 & 3.50 & 1.70 \\
\hline eadive & ivis.s & 2004.00 & 1750.00 & 100.00 & 9.60 & 396.32 \\
\hline inollin & 0.6 & 0.90 & 1.90 & $\cdots$ & 1.70 & 1.30 \\
\hline Venediu & 4.7 & 6.14 & 13.7 & 30.20 & 31.10 & 1.46 \\
\hline $21 n$ & 340.7 & 280.14 & 266.00 & 174.12 & 127.60 & 120.71 \\
\hline Grentes & $m$ & $m$ & in & $m$ & in & inn \\
\hline
\end{tabular}

worle All extals ore repored in m/kg.

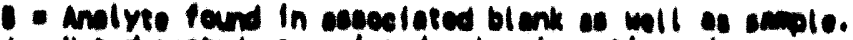

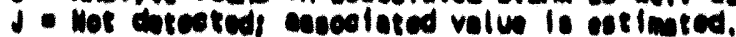

in - conreporteble.

- Dote ore unucble.

$U$ - Hot eseceted voliu reported is ecople auntitotion IInte.

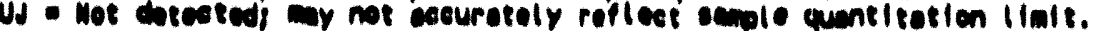


Table 10. Average Values of all Samples Collected in 1991 and 1992 as Part of 100 Areas Ecological Investigations and Other Studies. (sheet 2 of 2)

\begin{tabular}{|c|c|c|c|c|}
\hline \multirow{3}{*}{ constitumt } & \multicolumn{4}{|c|}{ sepple Identiflestion Mubers } \\
\hline & 27 & $r$ & 17 & 22 \\
\hline & Ennoy & anase & AcPraness & Ants \\
\hline Aluatrus & 720.74 & 249.69 & 27.91 & 549.09 \\
\hline Antionan & 3.26 & 4.24 & 0.25 & 3.22 \\
\hline Areente & 2.02 & 0.01 & 2.4 & 1.80 \\
\hline Derlin & 210.12 & 22.28 & 4.01 & 110.18 \\
\hline cervillen & 0.49 & 0.18 & 0.49 & 0.40 \\
\hline contive & 0.19 & 0.32 & 1.08 & 0.19 \\
\hline colelin & 6000.76 & 4226.76 & 1638.00 & 9220.98 \\
\hline chrealen & 0.34 & 1.67 & 2.7 & 7.01 \\
\hline cobale & 0.68 & 0.76 & 1.29 & 6.94 \\
\hline cepper & 14.93 & 6.93 & 0.16 & 14.03 \\
\hline Irmen & 17170.37 & 449.42 & 71.11 & 10990.91 \\
\hline leet & 6.50 & 1.30 & 0.82 & 7.30 \\
\hline necreselon & 431.40 & 2092.60 & 1031.20 & 3ex.c4 \\
\hline nenerenese & 81.03 & 52.93 & 10.32 & 203.45 \\
\hline morouny & 0.05 & 0.00 & 0.23 & 0.08 \\
\hline Wlekel & 9.00 & 1.93 & 2.01 & 0.00 \\
\hline Potese In & 1430.11 & 15709.83 & 22370.99 & 1396.4 \\
\hline celentur & 1.03 & 1.60 & 2.17 & 0.70 \\
\hline sllver & 0.76 & 0.75 & 1.60 & 0.48 \\
\hline sedive & 309.88 & 91.69 & 194.27 & 223.91 \\
\hline Thallin & 0.55 & 1.4 & 2.16 & 0.69 \\
\hline Venediv & 39.40 & 1.04 & 1.16 & 39.15 \\
\hline zine & 9.92 & 0.61 & 97.65 & 41.02 \\
\hline Grentes & Nh & Mn & inn & NR \\
\hline
\end{tabular}

worle All motals ore reported in ma/kg.

\footnotetext{
- Anolve found In assoclated blank as mall os semple.

J- Mot detected asseclated valus lo estimeted.

in - Menreporteble.

- Doto are unucble.

$U$ - Mot detested value reported le semple auentitation IIalt.

UJ - Not datestedi may not eccurotely reflect semple quantitotion IImits.
} 
Table 11. Average Values for Sediments Collected in the 100 Areas".

\begin{tabular}{|c|c|c|c|c|c|c|c|}
\hline \multirow{2}{*}{ Conetitumt } & \multicolumn{7}{|c|}{ Resctor slte supple locut lons } \\
\hline & ec & $\mathbf{k}$ & $n$ & 0 & H & 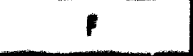 & Men. Tow \\
\hline Alvinn & 3916.00 & 5777.00 & 9604.00 & 6200.00 & 6632.00 & 6051.00 & 0327.00 \\
\hline Antinemy & 12.30 Ud & $10.00 \mathrm{Ud}$ & $0.20 \mathrm{us}$ & 6.95 UJ & 12.80 us & $10.20 \mathrm{us}$ & $0.20 \mathrm{UJ}$ \\
\hline Derive & $95.20 \mathrm{I}$ & $6.30 \mathrm{~J}$ & 7.06 & 71.10 & $61.04 \mathrm{~J}$ & $59.80 \mathrm{~J}$ & 86.70 \\
\hline eorylliun & $0.33 \mathrm{~V}$ & $0.27 \mathrm{~V}$ & $0.22 \mathrm{v}$ & $0.25 \mathrm{~V}$ & $0.34 \mathrm{U}$ & $0.36 \mathrm{U}$ & $0.40 \mathrm{U}$ \\
\hline cocialun & $0.83 \mathrm{v}$ & $0.69 v$ & $0.59 \mathrm{U}$ & $1.08 \mathrm{us}$ & $0.81 \mathrm{U}$ & $0.95 \mathrm{U}$ & $1.70 \mathrm{u}$ \\
\hline cololum & 3150.00 & $31 \% .00$ & 3152.00 & 4530.00 & $4256 \cdots$ & $4033.00 \mathrm{~J}$ & 4600.00 \\
\hline Chrealu & $46.90 \mathrm{~J}$ & 34.60 & $11.64 \mathrm{~J}$ & $76.05 \mathrm{~J}$ & $68.70 \mathrm{~J}$ & $15.30 \mathrm{~J}$ & 18.70 \\
\hline cemale & $3.70 \mathrm{~d}$ & 0.000 & 7.70 & 0.090 & 7.700 & $7.50 \mathrm{D}$ & 10.00 \\
\hline Cepper & 19.30 & $10.20 \mathrm{~J}$ & $16.50 \mathrm{~J}$ & 14.40 & $26.00 \mathrm{~J}$ & $18.90 \mathrm{~J}$ & $23.10 \mathrm{~J}$ \\
\hline Iren & 12700.00 & 16700.00 & 13940.00 & 92600.00 & 16040.00 & $49485.00 \mathrm{~J}$ & 26734.00 \\
\hline moenesive & 3404.00 & 3220.00 & 3269.00 & 3560.00 & 3924.00 & 6056.00 & 6390.00 \\
\hline Anongenese & 180.30 & $353.00 \mathrm{~J}$ & $608.00 \mathrm{~J}$ & $370.00 \mathrm{~J}$ & $294.20 \mathrm{~J}$ & $266.00 \mathrm{~J}$ & $341.00 \mathrm{~J}$ \\
\hline NIckel & 11.600 & 12.50 & 11.90 & 9.90 & 12.18 & 11.70 & 17.10 \\
\hline Potenelin & $907.30 \mathrm{~J}$ & 691.001 & $655.20 \mathrm{D}$ & $668.00 \mathrm{U}$ & 703.208 & 740.00 & 1038.000 \\
\hline sllver & $1.20 \mathrm{U}$ & $0.92 \mathrm{~V}$ & $0.96 \mathrm{u}$ & 0.80 & $1.09 \mathrm{U}$ & $1.06 \mathrm{U}$ & $2.03 \mathrm{U}$ \\
\hline sediun & $140.00 \mathrm{~J}$ & $17.00 \mathrm{~J}$ & $152.80 \mathrm{~J}$ & $170.30 \mathrm{U}$ & $233.80 \mathrm{~J}$ & $211.00 \mathrm{~J}$ & $239.00 \mathrm{~J}$ \\
\hline Venedica & 29.60 & 33.20 & 37.68 & $31.70 \mathrm{~J}$ & 32.32 & 60.80 & 6.30 \\
\hline $21 \mathrm{ne}$ & 128.10 & $17.70 \mathrm{~J}$ & $117.02 \mathrm{~J}$ & $163.00 \mathrm{~V}$ & 237.00 & $133.00 \mathrm{~J}$ & 262.00 \\
\hline $60 \cdot 137$ & 0.15 & 0.18 & 0.16 & 0.23 & 0.26 & 0.32 & 0.36 \\
\hline $8 r \cdot 90$ & 0.30 & 0.66 & 46.20 & -0.04 & 0.72 & 8.80 & 1.40 \\
\hline
\end{tabular}

norts All meale are reported in mo/kg. Radionucllose are reported in pcllem.

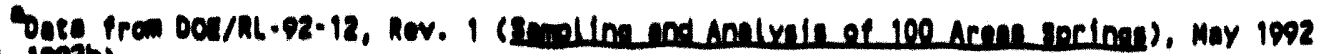
$(00 \mathrm{Ant} 1 \mathrm{mab})$.

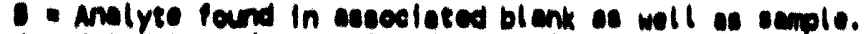

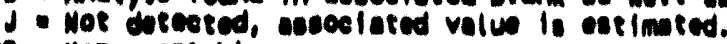

Na - venreperteble.

- Date are unucbile.

$U$ - Mot dateeted, valiu reported la semple quentitation IInle.

UJ - Not dotected, nay not oceurotely roflect emple quantitation IImit. 
WHC-EP-0620

Table 12. Soll Background Values (ppm) for Selected Metals Reported for the Hanford Sitea .

\begin{tabular}{|c|c|c|c|c|c|c|c|}
\hline \multirow{2}{*}{ Const i tuent } & \multicolumn{7}{|c|}{ Sample Identification Numbers } \\
\hline & 8002H9 & $8002 \mathrm{JO}$ & Bo0zJ1 & $8002 \mathrm{~J} 2$ & MIN & $\max$ & AVG \\
\hline A: uninu & 7100 & 7800 & 8100 & 8100 & 7100 & 8100 & $\pi 75$ \\
\hline Bariun & 80 & 88 & 96 & 89 & 80 & 96 & 88.25 \\
\hline Calcium & 3600 & 3600 & 4900 & 5100 & 3600 & 5100 & 4300 \\
\hline Chromiun & 8 & 9 & 9 & 8 & 8 & 9 & 8.5 \\
\hline Cobalt & 12 & 11 & 13 & 13 & 0 & 13 & 9.25 \\
\hline Copper & 9 & 11 & 14 & 13 & 9 & 14 & 11.75 \\
\hline Iron & 23000 & 22000 & 24000 & 24000 & 22000 & 24000 & 23250 \\
\hline Lead & 6 & 7 & 6 & 6 & 6 & 7 & 6.25 \\
\hline Magnesive & 3900 & 4000 & 4600 & 4800 & 3900 & 4800 & 4325 \\
\hline Mangenese & 340 & 360 & 410 & 360 & 340 & 410 & 367.5 \\
\hline Nickel & 8 & 9 & 11 & 10 & 8 & 11 & 9.5 \\
\hline Potasefium & 1500 & 1600 & 1400 & 1300 & 1300 & 1600 & 1450 \\
\hline Sodiun & 150 & 160 & 170 & 180 & 150 & 180 & 165 \\
\hline strontiv & 20 & 21 & 22 & 23 & 20 & 23 & 21.5 \\
\hline Thollium & No & No & No & NO & 0 & 0 & 0 \\
\hline Venadiun & 64 & 59 & 63 & 63 & 59 & 64 & 62.25 \\
\hline Zine & 43 & 44 & 42 & 42 & 42 & 44 & 42.75 \\
\hline
\end{tabular}

DOE/RL-88-41, ReV. 2, 1993. 
Table 13. Soll Threshold Values (ppm) and Cleanup Standards.

\begin{tabular}{|c|c|c|c|c|c|c|}
\hline \multirow{2}{*}{ Analyte } & \multicolumn{2}{|c|}{$\begin{array}{l}\text { Hanford soil } \\
\text { beckground }\end{array}$} & \multirow{2}{*}{$\begin{array}{l}\text { Ritzvillo } \\
\text { silt loan }\end{array}$} & \multicolumn{2}{|c|}{ World wide ${ }^{c}$} & \multirow{2}{*}{$\begin{array}{l}\text { Cleanup } \\
\text { stendard }\end{array}$} \\
\hline & & Maximum & & Mean & Range & \\
\hline Aluminum & 16,600 & 28,800 & 63,100 & 71,000 & $10,000-3000,000$ & \\
\hline Ant imony & 15.7 & 52.2 & 0.42 & 1 & $0.2 \cdot 10$ & 32 \\
\hline Arsenic & 9 & 32.5 & 4.6 & 6 & $0.1 \cdot 40$ & .58 \\
\hline Barium & 175 & 480.0 & 510 & 500 & $100-3,000$ & 5,600 \\
\hline Beryllium & 1.8 & 10 & 0.9 & 0.3 & $0.01 \cdot 40$ & .23 \\
\hline Cadnitum & 0.66 & 11 & 0.29 & 0.35 & $0.01 \cdot 2$ & 80 \\
\hline Calcium & 24,600 & 105,000 & 18,300 & 15,000 & $700-500,000$ & $\cdots$ \\
\hline Chromiun & 28 & 53 & 43 & 70 & $5-1,500$ & 8,000 \\
\hline Cobolt & 19 & 110 & 7.9 & 8 & $0.05 \cdot 65$ & $\cdots$ \\
\hline Coppar & 30 & 61 & 26 & 30 & $2 \cdot 250$ & 2,960 \\
\hline Iron & 38,200 & 68,100 & 43,700 & 40,000 & $2,000 \cdot 550,000$ & $\cdots$ \\
\hline Lead & 15 & 74 & 10 & 35 & $2 \cdot 300$ & 1,120 \\
\hline Magnesium & 9,160 & 32,300 & 11,200 & 5,000 & $400 \cdot 9,000$ & $\cdots$ \\
\hline Manganese & 580 & 1,110 & 620 & 1,000 & $20 \cdot 10,000$ & 16,000 \\
\hline Mercury & M/ & & 0.017 & 0.06 & $0.01-0.5$ & 24 \\
\hline Nickel & 25 & 110 & 29 & 50 & $2 \cdot 750$ & 1,600 \\
\hline Potassium & 3,090 & 7,900 & & & & \\
\hline selenium & 23 & & 0.30 & 0.4 & $0.01 \cdot 12$ & 240 \\
\hline silver & 2.1 & 4.5 & 0.10 & 0.05 & $0.01 \cdot 8$ & 240 \\
\hline sodium & 1,390 & 5,120 & 21,400 & 5,000 & $150 \cdot 25,000$ & $\cdots$ \\
\hline strontiun & 43 & & & & & \\
\hline Thallium & & & 0.33 & 0.2 & $0.1 \cdot 0.8$ & 5.6 \\
\hline Titanium & 3,307 & $\cdots$ & $\cdots$ & 5,000 & $150 \cdot 25,000$ & $\because$ \\
\hline Vanadium & 107 & 140 & 130 & 90 & $3 \cdot 500$ & 560 \\
\hline Zine & 79 & 366 & 70 & 90 & $1-900$ & 16,000 \\
\hline
\end{tabular}

Provisionsl threshold values besed on acid leach--U.S. Environmental Protection Agency (EPA) Methog 6010 (Hoover and Legore 1999).

Gildung et al. 1986.

Bowen 1979.

Model Toxic Control Act.

N/Av = Not available. 
Table 14. Maxima and 95/95 Reference Thresholds for Sitewide Soll Background ".

\begin{tabular}{|c|c|c|c|c|c|}
\hline \multirow{2}{*}{ Analyte } & \multicolumn{2}{|c|}{ Detection limits } & \multirow{2}{*}{$\begin{array}{c}95 / 95 \\
\text { threshold } \\
\text { (mg/kh) }\end{array}$} & \multirow{2}{*}{$\begin{array}{c}\text { Maximum } \\
\begin{array}{c}\text { concentration } \\
(\mathrm{mg} / \mathrm{kg})\end{array}\end{array}$} & \multirow{2}{*}{$\begin{array}{l}\text { Sample with maximum } \\
\text { concentration }\end{array}$} \\
\hline & LOD & LOQ & & & \\
\hline Aluminum & 21.8 & 66.1 & 15,100 & 28,800 & TOPSOIL, PLAYA, E-7 \\
\hline Ant imony & 15.7 & 52.2 & NC & 31 & VOLCANIC ASH \\
\hline Arsenic & $N / A$ & $N / A$ & 9.0 & 27.7 & TOPSOIL, JUNIPER, E-3 \\
\hline Barium & 0.87 & 2.7 & 175 & 480 & VOLCANIC ASH" \\
\hline Beryllium & $N / A$ & $N / A$ & 1.8 & 10 & VOLCANIC ASH \\
\hline Cadmium & 0.24 & 0.79 & NC & 11 & VOLCANIC ASH" \\
\hline Calcium & 175 & 470 & 24,600 & 105,000 & TOPSOIL, GREASEWOOD, E-2 \\
\hline Chromium & 1.1 & 3.0 & 28 & 320 & RINGOLD FM" \\
\hline Cobalt & 0.88 & 2.9 & 19 & 110 & VOLCANIC ASH \\
\hline Copper & 2.1 & 6.2 & 30 & 61 & VOLCANIC ASH \\
\hline Iron & 75.7 & 236 & 38,200 & 38,100 & RINGOLD FM \\
\hline Lead & $N / A$ & $N / A$ & 14.9 & 74.1 & TOPSOIL, JUNIPER, E-3 \\
\hline Magnesium & 18.4 & 57.9 & 9,160 & 32,300 & TOPSOIL, GREASEWOOD, E-2 \\
\hline Manganese & 0.63 & 1.8 & 583 & 1,110 & TOPSOIL, PLAYA, E-7 \\
\hline Mercury & $N / A$ & $N / A$ & 1.3 & 3.8 & RANDOM SAMPLES, \#15 \\
\hline Nickel & 2.4 & 7.7 & 25 & 200 & RINGOLD FM" \\
\hline Potassium & 135 & 451 & 3,090 & 7,900 & TOPSOIL, PLAYA, E-7 \\
\hline Selenium & $N / A$ & $N / A$ & $N C$ & 6 & RANDOM SAMPLES, \#15 \\
\hline Silver & 2.1 & 4.5 & 2.1 & 14.6 & RANDOM SAMPLES, \#6 \\
\hline Sodium & 50.6 & 140 & 1,390 & 6,060 & RANDOM SAMPLES, \#12 \\
\hline Thallium & $N / A$ & $N / A$ & NC & 3.7 & LAB DETECTION LIMIT \\
\hline Vanadium & 1.8 & 5.9 & 107 & 140 & VOLCANIC ASH \\
\hline Zinc & 6.4 & 15.6 & 79 & 366 & TOPSOIL, JUNIPER, E-3 \\
\hline
\end{tabular}


between the threshold values for various metals, which indicates the need to develop some standard references for metal background values at the

Hanford Site. Because other Hanford Site documents, such as Peterson and Johnson (1992), used the soll threshold values reported by Hoover and Legore (1991) (DOE-RL 1993), this report also uses them for data comparison. Maximum values of heavy metals concentrations that were collected at the Hanford Site (Hoover and Legore 1991) are provided in Table 14 to indicate the wide variation of concentration values and to show that values that may exceed the threshold values are not necessarlly cause for concern. The soll threshold values reported by Hoover and Legore (1991) represent the upper limit of the 95\% confidence level.

Only recent information exists at the Hanford Site for metals concentrations in flora and fauna. Some limited data is avallable on metals in phytoplankton and caddis flles collected from the Columbia River (Cushing 1979 and 1993) and some information regarding uptake of metals by plants and animals in the 300 Areas (300-FF-1) at the Hanford Site (Brandt and Rickard 1992). The latest information (Cushing 1993) regarding uptake of metals by aquatic organisms showed undetectable levels of chromium in caddis fly larvae and chromium levels that were essentlally the same for samples collected at the 100 Areas operable units and control samples. For reference purposes, the heavy metals in vegetation presented in this report can al so be compared with plant toxicity levels for selected metals in Table 15.

Vegetation samples collected in the 300-FF-1 operable unit (Brandt and Rickard 1992) that were analyzed for metals indicated very low levels. A summary of the findings in that study indicated that direct human health risk from metals in vegetation outside exclusion fences is minimal.

Some plants and animals do select and/or concentrate various metals and other inorganic constituents based on life history phenomena, soll type, etc. (Adriano 1986). An example of differential uptake is in earthworms, which can concentrate heavy metals from soils many times the soil metal levels. Earthworms found in soils with $2.0 \mathrm{ppm}$ of cadmium contained $100 \mathrm{ppm}$ of the heavy metal (Beyer et a1. 1982). Another 11lustration of the large amount of varlability in uptake of metals between species is shown in Table 15 (regarding toxicity levels). Some species can tolerate much higher levels of various metals than others.

Radionuclide data are compared with soll and vegetation samples collected by Westinghouse Hanford as part of the operational monitoring program in the 100 Areas from 1981 to the present (Appendix H) and with uranium values in asparagus collected in the 300 Areas (Tiller and Poston 1992). Radionuclide levels in trees also are compared with previous radionuclide uptake studies by trees at the Hanford Site (Landeen and Mitche11 1986 and Rickard and Price 1989).

\subsection{SOURCES OF METALS AND RADIONUCLIDES}

Possible sources of metals and radionuclides found at the Hanford Site that could explain some of the values observed in this and other reports are discussed in Peterson and Johnson (1992). The following information that 
WHC-EP-0620

Table 15. Toxicity Levels of Selected Metals in Plants. (sheet 1 of 2)

\begin{tabular}{|c|c|c|c|}
\hline Constituent & $\begin{array}{l}\text { Plant } \\
\text { spectes }\end{array}$ & $\begin{array}{c}\text { Toxic } \\
\text { levels (ppm) }\end{array}$ & Reference \\
\hline \multirow[t]{9}{*}{ Zlnc } & corn & $>450$ & Gall and Barnette 1940 \\
\hline & cowpeas & $>180$ & Gall and Barnette 1940 \\
\hline & cotton & 200 & Chapman 1966 \\
\hline & tomatoes & 526 & Chapman 1966 \\
\hline & oats & 1,700 & Ohki 1975 \\
\hline & apples & $>100$ & Benson 1966 \\
\hline & lettuce & $>500$ & MacLean 1974 \\
\hline & alfalfa & $>700$ & MacLean 1974 \\
\hline & peas & $>50$ & Melton et al. 1970 \\
\hline \multirow[t]{6}{*}{ Cadmium } & spinach & $4($ in soil) & Adriano 1986 \\
\hline & rice & $>600($ in soll) & Adrtano 1986 \\
\hline & corn & $>2.5($ ln soli) & Mtller et a1. 1977 \\
\hline & soybeans, wheat & $>2.5($ in soll $)$ & Haghiri 1973 \\
\hline & clover & $>5($ in soll) & Will Iams and David 1977 \\
\hline & barley & 15 & Dav1s et al. 1978 \\
\hline \multirow[t]{4}{*}{ Chromium } & barley, corn & 5 & Pratt 1966 \\
\hline & oats, citrus & & \\
\hline & tobacco & 175 & Pratt 1966 \\
\hline & rice & $>35$ & Chino 1981 \\
\hline \multirow[t]{3}{*}{ Copper } & rice & $>20$ & Chino 1981 \\
\hline & citrus & $>150($ in soli) & Baker 1974 \\
\hline & snapbeans & $>20($ in soil) & Walsh et al. 1972 \\
\hline \multirow[t]{3}{*}{ Lead } & barley & $>35$ & Davis et al. 1978 \\
\hline & rice & $>50$ & Chino 1981 \\
\hline & Japan plants & $>400($ in soll) & Chino 1981 \\
\hline \multirow[t]{4}{*}{ Manganese } & apple & $>400($ in soll $)$ & NAS 1973 \\
\hline & Wheat & $>350($ in soll) & NAS 1973 \\
\hline & alfalfa & $>477$ & NAS 1973 \\
\hline & barley & $>300$ & NAS 1973 \\
\hline \multirow[t]{5}{*}{ Nickel } & general plants & $>50$ & Adriano 1986 \\
\hline & ryegrass & $>90($ in soill) & Khalid and Tinsley 1980 \\
\hline & rice & $20-50$ & Chino 1981 \\
\hline & barley & 26 & Davis et al. 1978 \\
\hline & hardwoods & $100-150$ & Lozano and Morrison 1981 \\
\hline
\end{tabular}


WHC-EP-0620

Table 15. Toxicity Levels of Selected Metals in Plants. (sheet 2 of 2)

\begin{tabular}{|c|c|c|c|}
\hline Constituent & $\begin{array}{c}\text { Plant } \\
\text { Species }\end{array}$ & $\begin{array}{c}\text { Toxic } \\
\text { Levels (ppm) }\end{array}$ & Reference \\
\hline \multirow[t]{6}{*}{ Selentum } & Wheat & $40($ in soll) & Beath et al. 1937 \\
\hline & cotton & $20($ In soll) & Adriano 1986 \\
\hline & fescue & 117 & Walsh and Fleming 1952 \\
\hline & meadow sweet & 30 & Walsh and Fleming 1952 \\
\hline & barley & $>7$ & Walsh and Fleming 1952 \\
\hline & alfalfa & $>2(\ln 5011)$ & Soltampour and Workman 1980 \\
\hline \multirow[t]{2}{*}{ Bartum } & bush beans & $>2,000($ in sol1) & Chaudry et al. 1977 \\
\hline & barley & & \\
\hline \multirow[t]{2}{*}{ Sllver } & Larley & 4 & Davis et al. 1978 \\
\hline & bush beans & $>5$ & Wallace et al. 1977 \\
\hline \multirow[t]{2}{*}{ Thallium } & crops & $>7$ & Carson and Smith 1977 \\
\hline & tobacco & $>1($ in soli) & Carson and Smith 1977 \\
\hline Vanadium & plants in general & $>1$ & Davis et al. 1978 \\
\hline
\end{tabular}


discusses these sources was obtained from their report. Concentrations of some metals in sediments found in the 100 Areas may be influenced by upstream sources such as mining and related refining activities in the upper Columbia River drainages. These areas include Sullivan, British Columbia, Republic, and Metaline, Washington, as well as Coeur d'Alene, Idaho. Above-background occurrences of such metals as lead, 2 inc, copper, and cadmium may come from these sources (Maxfield et al. 1974; Johnson 1991 and 1979; Rember et al. 1991; Robbins 1978; Sllker 1964; and Miller et al. 1975). Metals such as antimony, thallium, arsenic, and manganese also are associated with British Columbia mining activities (Hoy et al. 1985). Studies conducted behind Priest Raplds and McNary dams (Whetten et al. 1969) showed that zinc concentrations were approximately eight to five times higher than natural background. Similar patterns were observed for lead, cadmium, and copper (Whetten et al. 1969). Chromtum concentrations espectally at 100-D and 100-H reactors are probably the result of past liquid disposal practices associated with the reactors.

Peterson and Johnson (1992) al so reported elevated levels of strontlum-90 in seepage at $100-N, 100-K$, and $100-H$ reactors, the probable cause of the elevated strontfum-90 leveis in trees at $100-K$ reactor reported in the following sections.

Appendix $J$ graphically shows average values for selected metals in all media, including control values collected as part of the 100 Areas ecological surveys and other similar sampling efforts. These figures are included to provide perspective and comparisons of the metals data that has been collected at the Hanford S1te.

\subsection{ASPARAGUS}

The analytical results from the asparagus sampling are given in Tables B-1 and B-2 in Appendix B. Results from this effort show that levels of metals and radionuclides collected in 1991 and 1992 in asparagus are very low, with the possible exception of zinc. Zinc concentrations from asparagus collected at 100-F indicated levels up to $97 \mathrm{ppm}$, which is higher than the soll threshold value of $79 \mathrm{ppm}$ (Figure 1). However, the average values of zinc in asparagus collected in the 100 Areas and the offsite control samples were essentialiy identical (59 and $58 \mathrm{ppm}$, respectively). These results are also similar to the zinc values reported in tree leaves collected in 1991 and inflfoll collected at $N$ Springs (see Section 8.4). In both instances, the zinc values were higher than the soll threshold limit of $79 \mathrm{ppm}$. Toxic levels of zinc from Table 15 in plants indicates concentrations ranging from $50 \mathrm{ppm}$ in peas to $1700 \mathrm{ppm}$ in oats. There were no visible signs of stress assoctated with the asparagus sampled in this study.

Chromium concentrations were very low in asparagus, with the majority of the results undetectable. Chromium is a metal of concern at the Hanford Site and has been detected in groundwater samples from the 100 Areas (Peterson 1991, Peterson and Connelly 1992). Chromium seems to be translocated poorly in plants, and "normal" soils concentrations in plants are usually less than 1 ppm (Pratt 1966). 
WHC-EP-0620

Figure 1. 2inc Concentration in Asparagus (1992).

ZIne Concentration In Asparagus Colloctod in 1992.

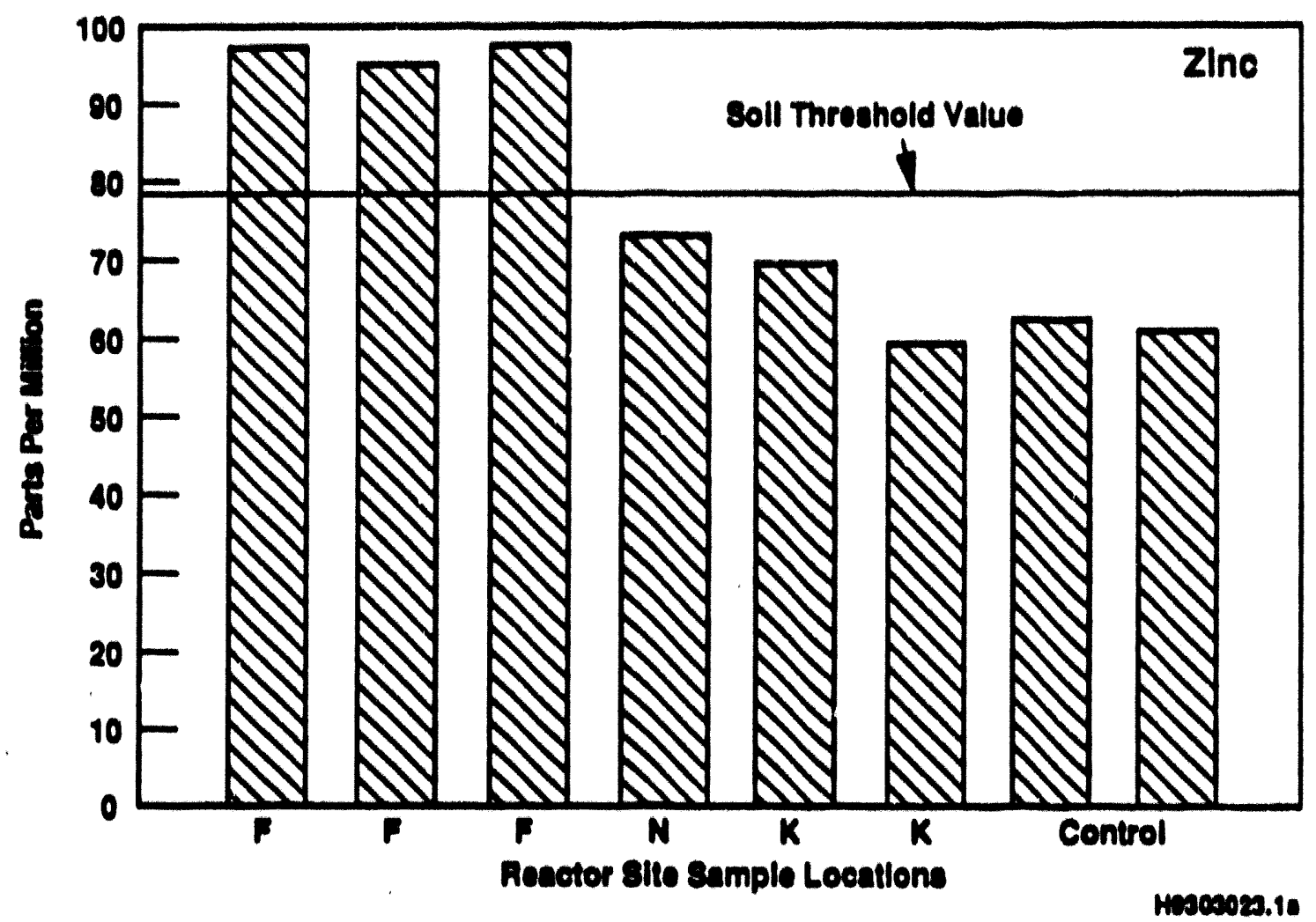


Average aluminum, bartum, lead, Iron, manganese, magnesfum, and vanadium concentrations in asparagus were the lowest for 11 medla sampled (Appendix J). Potasslum concentrations in asparagus, including the controls, were among the highest levels observed, second only to trees.

Technet fum-99, stront lum-90, and cesium-137 were below detection 1 imits in asparagus. The only other radionuclide data avallable in asparagus from the Hanford site are uranium concentrations from the 300 Areas (T11) Poston 1992), which were low.

\subsection{REED CAMARYenass}

The analytical results for reed canarygrass are given in Tables $C-1$ through $\mathrm{C}-13$ of Appendix $\mathrm{C}$. Metals concentrations in reed canarygrass were among the lowest of all the vartous medla that were sampled (Appendix $J$ ) and exhlbited the same basic trends as asparagus. For the majority of the metals, reed canarygrass values were higher in the control samples. Reed canarygrass, like many of the media, showed levated levels of zinc compared to the soll threshold level of $79 \mathrm{ppm}$. However, the majority of all the medla sampled (including the controls) showed this same trend, indicating that these levels of zinc are probably normal for vegetation growing in the Hanford site environs.

Radionuclide values for cestum-139, stront fum-90, and technet fum-99 were very low or undetectable and very rarely exceeded $1 \mathrm{pC} / \mathrm{gm}$. These values are comparable to or less than the values reported in vegetation from the 100 Areas is part of Westinghouse Hanford's rout ine monitoring program (Appendix H).

\subsection{TREE LEAVES NO LIMAS}

The analytical results from the tree leaves are given in Tables $D-1$ through $0-3$ of Appendix $D$. Analyt 1 cal results from tree leaves collected in 1991 at $100-0,100-H$, and $100-B C$ reactors showed up to $2.4 \mathrm{pC} 1 / \mathrm{g}$ stront 1 um-90 and $2.0 \mathrm{pCl} / \mathrm{g}$ technet lum-99. Tree leaves collected in July of $1992 \mathrm{did}$ show levated levels of stront lum-90 up to $35 \mathrm{pCl} / \mathrm{g}$ (Table D-2) from samples collected near 100-K reactor. To verify these results, a follow-up sampling effort was conducted in October 1992. Twelve samples of tree 1 imbs and leaves were sampled in the vicinities of $B C$ and $K$ reactors from six mulberry trees, including the same tree that had a measured strontium-90 concentration of $35 \mathrm{pCl} / \mathrm{gm}$. The follow-up samples from this same tree indicated strontium-90 levels of $43.0 \mathrm{pCl} / \mathrm{gm}$ in the limbs and $88.0 \mathrm{pCl} / \mathrm{gm}$ in the leaves. Two other trees sampled below $B C$ reactor had stront lum-90 levels ranging from $7.1 \mathrm{pCl} / \mathrm{gm}$ to $23.0 \mathrm{pCl} / \mathrm{gm}($ Table $\mathrm{D}-3)$. These results are graphed in figure 2.

Other Hanford Stte studtes have been conducted to record cestum-137 and stront fum-90 levels in trees. Landeen and Mitchell (1986) sampled trees at 216-U-10 Pond before it was decommisstoned and found levated levels of these radionuclides in the roots, cores, and leaves. The levels they reported for these radionuclides were higher than anything found in the trees growing along 
the Columbla River. Rlckard and Price (1989) analyzed tritfum concentrations in locust trees near $K$ reactor and found levels comparable to those reported in this report.

Flgure 3 shows levels of copper and 2 inc in tree leaves. Tree leaves collected in 1991 had highar levels of both 2 inc and copper than tree leaves that were collected in 1992. Copper is one of the seven micronutrients essentlal for normal plant nutrition. Kubota (1983) conducted a nationwide survey and found average levels of $8.4 \mathrm{ppm}$ in legumes and $4 \mathrm{ppm}$ in grasses. Studies of copper distributions and levels in forest ecosystems have shown that copper tends to accumulate in the organic matter. One sweden study (Tyler 1972) of a forest ecosystem had copper values ranging from 2 to 660 ppm.

In general, watals concentrations in tree control samples were higher than those collected on the Hanford site, with the exception of magnes um and potasstum. Trees had highor concentrations of magnestum and potasstum than any other madium (Appendix J).

\subsection{MATER MILFOIL}

The analytical results from the three water milfoll samples are provided in Table D-4 of Appendix 0 . Milfoll is an aquatic plant that may have more opportunity to take up hazardous waste constituents from the river sedimants and water. Mllfoll was one of the few modia that had higher values for the majority of the motals in the Hanford site samples than in the control samples. Cadmium, lead, copper, and manganese concentrations in inflfotl were higher than for all other madta sampled in the 100 Areas, and concentrations of nickel in milfoll were second only to the 100 Areas river sediments (Appendix J). Sodium concentrations were the highest for all vegetation samples. The results also indicated that the levels of antimony, cadmium, lead, and zinc sometimes exceeded the soll threshold values from Table 14 including the control samples (Figure 4). The antimony values were all data quallfied as undetectable.

These results may be considered normal for milfoll; however, as already indicated, no background values are avallable for milfoll at the Hanford site. Also, these results could indicate possible contamination from outside sources as discussed in Section 8.1.

The average concentration of zinc values in milfoll [246 ppm (Table D-4)] are simflar to the average zinc concentrations in Columbla River sediments (174 ppm) collected in 1991 (DOE-RL 1992b), which may indicate a correlation botween aquatic species, such as milfoll, and the river sediments.

Normally, cadmium is taken up in small amounts by plants. Normal levels of cadmium in food crops is around $0.5 \mathrm{ppm}$ (Friberg et al. 1971). Fol lage of sugar maple in Now Hampshire and Vermont had cadmium concentrations in the range of 0 to $5 \mathrm{ppm}$ (Smith 1973). Plants growing in areas of the world with soll known to be contaminated with cadmium have been documented to contain levels up to $600 \mathrm{ppm}$ (Adriano 1986). 
WHC-EP-0620

Figure 2. Stront lum-90 Concentrations at Reactor sites (1992).
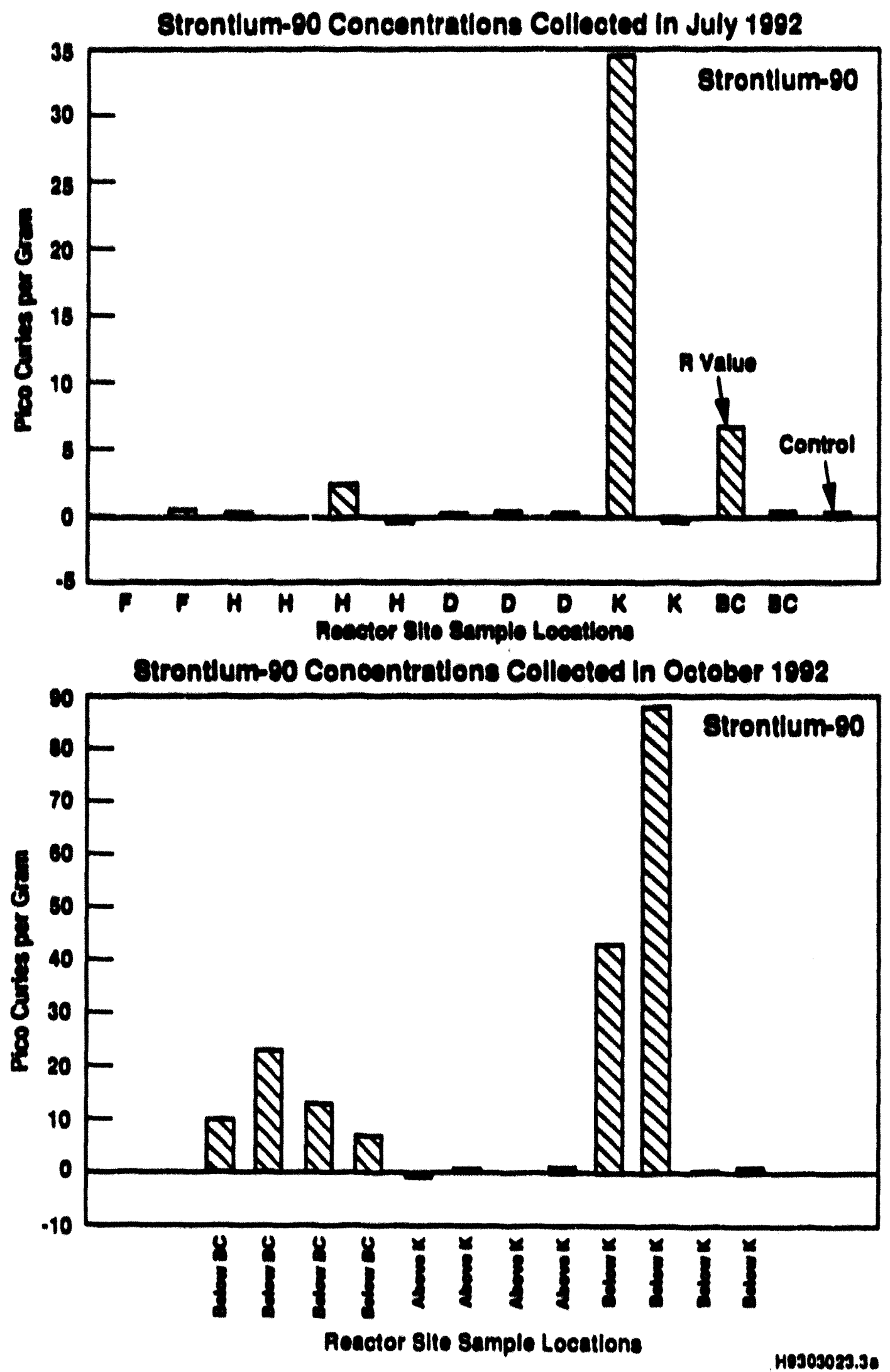
WHC-EP-0620

Figure 3. Copper and 2 inc Concentrations at Reactor Sites (1991 and 1992).
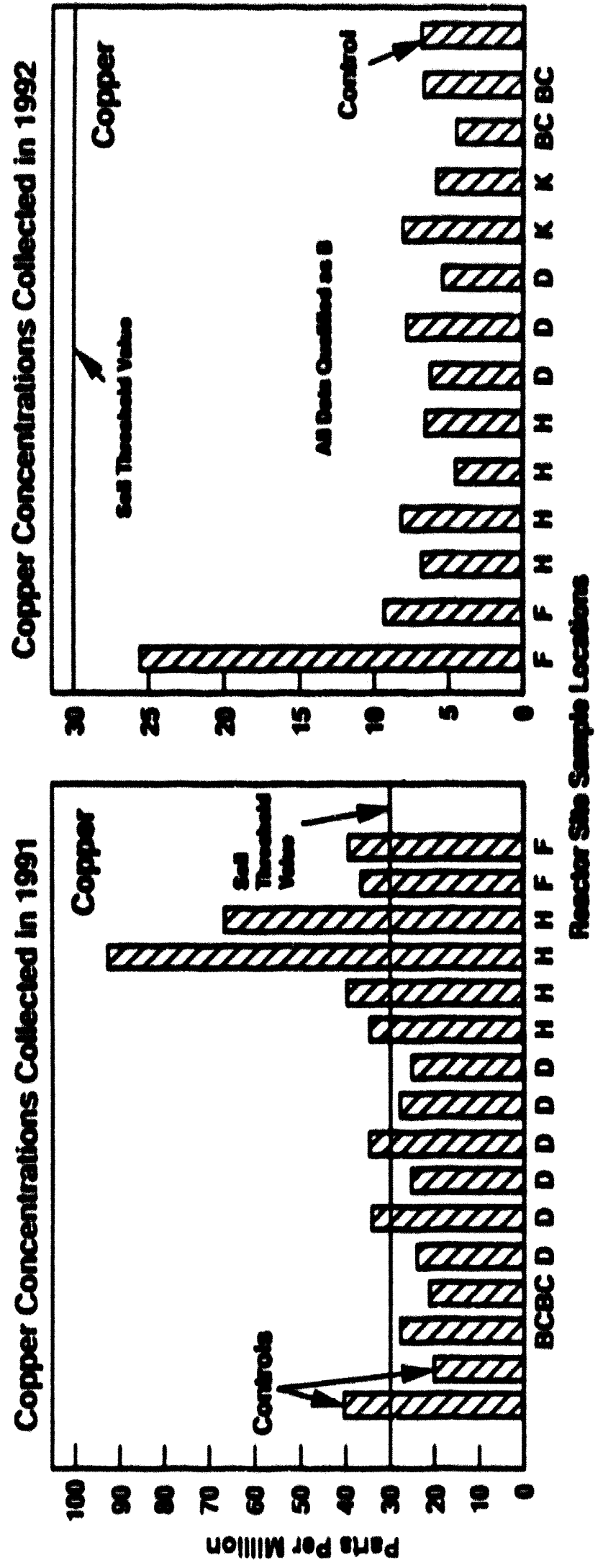
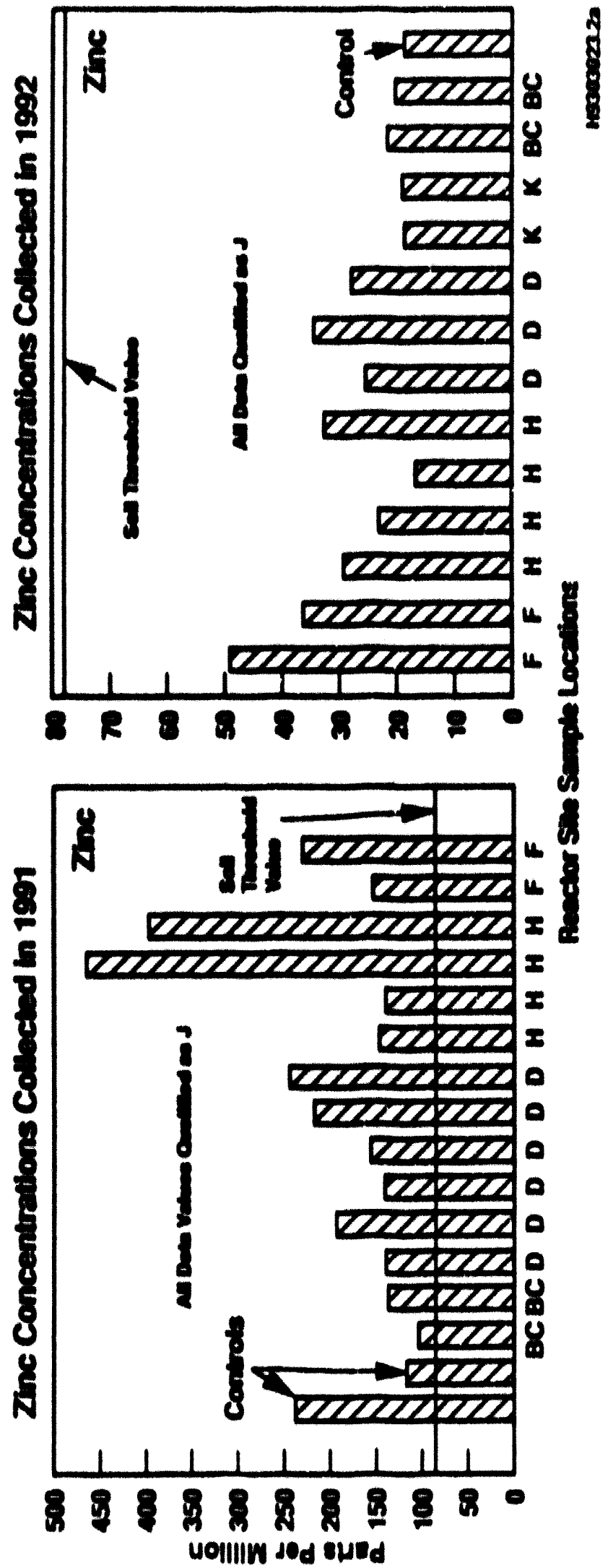
WHC-EP-0620

Figure 4. Antimony, Cadmlum, Lead, and 2 inc Concentrations at N Springs (1992).
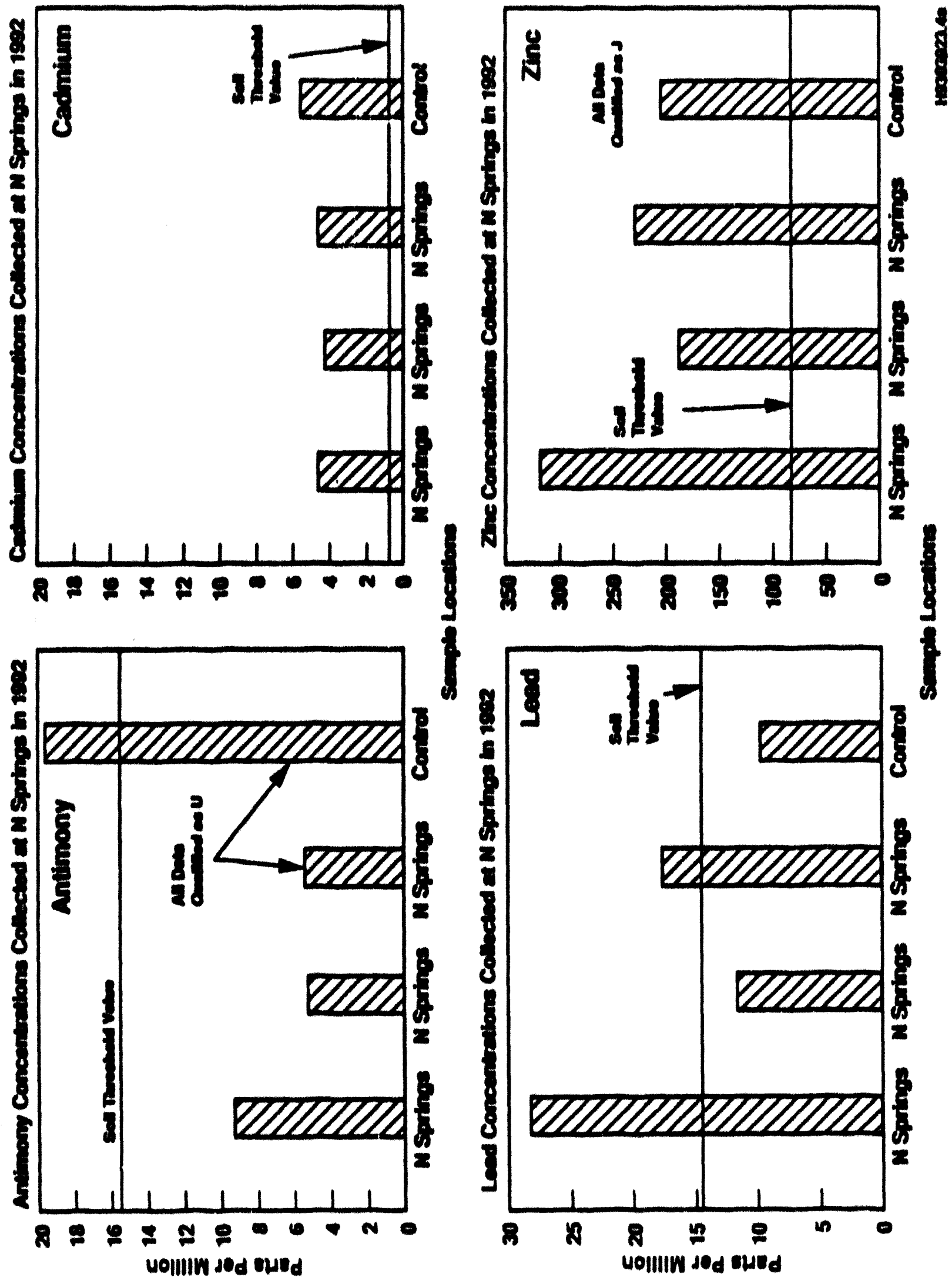
Lead concentrations in plants can be attributed to root uptake and external deposition. Plants growing in solls known to have high lead concentrations also can have high lead concentrations. Marten and Hammond (1966) showed uptake of lead by bromegrass in sandy solls up to $680 \mathrm{ppm}$.

The general knowledge regarding antimony levels in solis and plants is minimal. Normal antimony levels in most terrestrial plants seem to be around <0.1 ppm (Adrlano 1986).

Strontlum-90 concentrations in milfoll ranged from 0.18 to $0.61 \mathrm{pCl} / \mathrm{gm}$ at $N$ Springs. Similar values were reported by Antonto et al. (1993) in milfoll at $100-N$ with values ranging from 0.11 to $0.14 \mathrm{pCl} / \mathrm{gm}$ of stront 1 um- 90 . Control values in both studies collected above Vernita Bridge ranged from -0.04 to $0.08 \mathrm{pCl} / \mathrm{gm}$. In this study, ceslum-137 was detected in only one sample at $0.19 \mathrm{pC} / \mathrm{gm}$.

\subsection{ANIMLLS (COYOTES NNO RAPTORS)}

Results from the coyote scat and raptor pellets indicated low levels of motals and radionuclides, except for of $z$ inc (Tables $E-1$ and $E-2$ of Appendix E). Zinc values in coyote scat and raptor pellets exceeded the soll threshold value of $79 \mathrm{ppm}$ (F/gure 5). The control samples also exceeded 79 ppm. which indicates that this may be a normal body burden based on the diet of this species. Coyotes are omnivorous and will eat a wide varlety of plant and animal species. Raptors, however, are almost exclusively carnivorous so the zinc values may represent normal values in the prey base. Vartous spectes of small mammals, snakes, and insects throughout the 100 Areas would have to be sampled to determine background levels of metals. Talmage and Walton (1991) found relationship between contaminants in soll or food and selected target tissues in small mammals. Heavy metals showed a close relationship, with the kidney being the best assay organ except for bone, which was best for lead.

The results from this study did not indicate that lead concentrations in raptors and coyotes were much different than for soll collected at $2101-M$ Pond on the 200 Areas plateau and in the ant mounds and small mamal burrows. However, average lead concentrations in both coyotes (7.83 ppm) and raptors (5.98 ppm) were higher than the control samples $(2.63 \mathrm{ppm}$ and $3.18 \mathrm{ppm}$, respectively) (Figure 5). Heavy metal concentrations of lead, cadmium, nickel, and zinc in smali mammals can be directly correlated with vehicle traffic volume (Scanion 1979). Another study by Wheeler and Rolfe (1979) showed that the distribution of lead in soll and vegetation along roadsides seemed to follow a double-exponentlal function. The first exponent is assoclated with large particles that settle out rapldiy, usually within $5 \mathrm{~m}$ of the highway; and the second exponent is assoclated with smaller particles that settled out more slowly, within about $100 \mathrm{~m}$ of the source. Some of the raptor pellets and coyote scat collected in the 100 Areas were along roads that are used frequently.

Average concentrations of aluminum, cadmium, chromium, cobalt, copper, Iron, load, magnesium, nickel, potassium, and vanadium were slightly higher in coyote scat than in the raptor pellets. Raptor pellets had higher concentrations of sodium and zinc than the coyote scat. 
$W H C-E P-0620$

Figure 5. Zinc and Lead Concentrations for Coyote Scat and Raptor Pellets (1992).

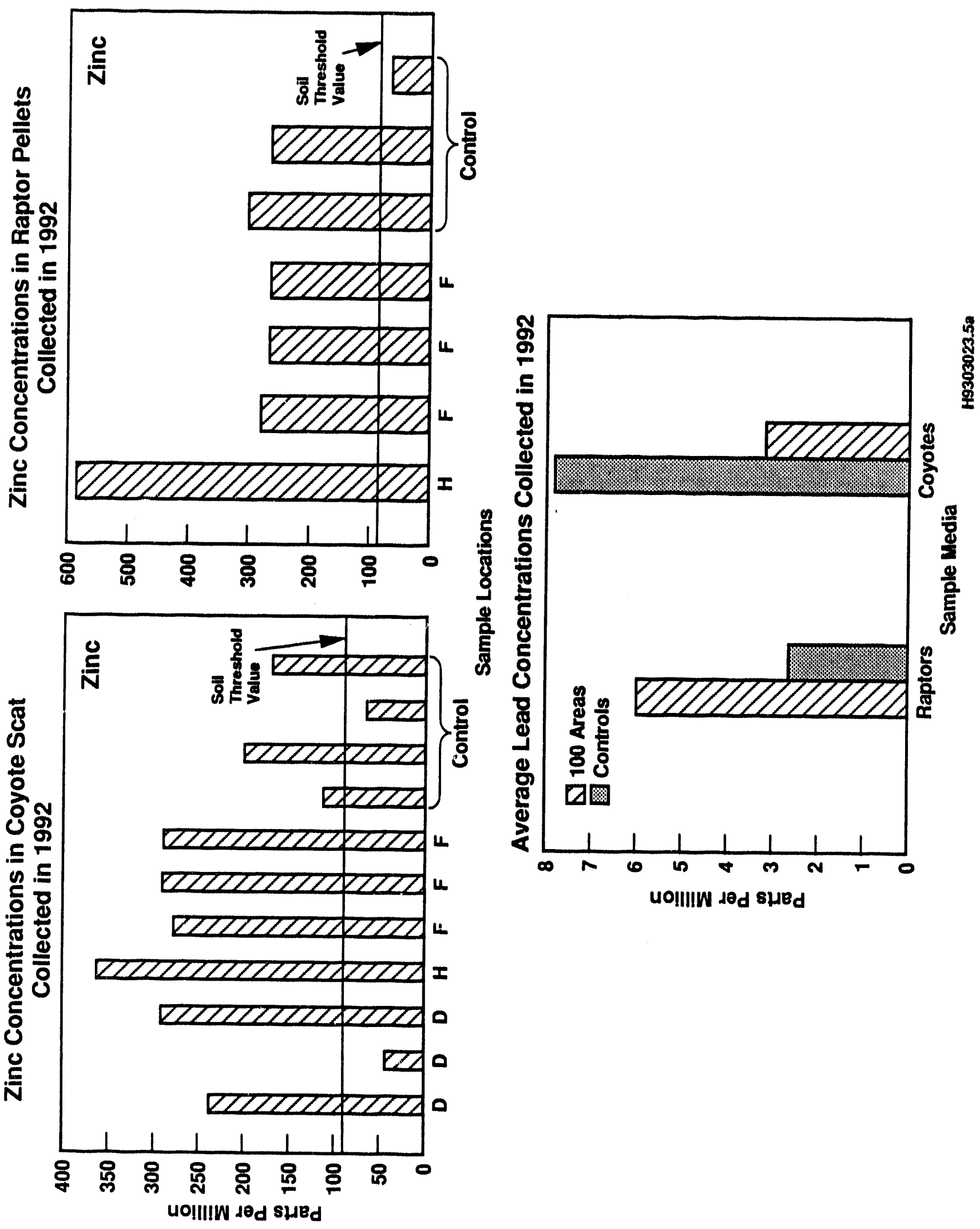




\subsection{ANT MOUNDS}

Tables showing the analytical results of the soil collected from the ant mounds are presented in Tables F-1 through F-5 of Appendix F. Results of the metals analyses indicated levels that were low or undetectable for many of the metals. The average concentrations in the control samples were higher than in samples collected on the Hanford Site.

Chromium levels in the ant mounds averaged $7.01 \mathrm{ppm}$, compared to an average of $35.96 \mathrm{ppm}$ reported from the Columbia River sediment sampling effort (DOE-RL 1992b) (Appendix J).

Radionuclide results for strontium-90 and cesium-137 were all undetectable or less than $1 \mathrm{pCi} / \mathrm{gm}$. The radionuclide levels here are comparable or less than the levels reported in soll by Westinghouse Hanford's routine monitoring program for the 100 Areas (Schmidt et al. 1992). Evidence from the limited number of ant mounds sampled from this study does not indicate that ants are bringing up radionculides and exposing them to the surface. However, a more intensive sampling effort would need to be conducted on any specific waste sites of interest.

\subsection{SMALL MAMMAL BURROWS}

The analytical results of the soil collected from the small mammal burrows are presented in Tables G-1 through G-6 of Appendix $G$. These results were very similar to the results reported for the ant mounds.

Small mammal burrows generally exhibited higher levels of aluminum and chromium compared to all other media sampled (Appendix $\mathrm{J}$ ), but the levels were still substantially below those considered to be of environmental concern. The chromium levels in the small mammal burrows were well below the average chromium value of $35.96 \mathrm{ppm}$ reported for the Columbia River sediments (DOE-RL 1992b). The average aluminum values for all the small mammal burrows including the control data are almost identical to those values reported as part of the 2101-M Pond study (Mitchell 1993).

The concentrations of cesium-137 and strontium-90 were very low or undetectable. Only one sample, collected at $\mathrm{N}$ reactor, exceeded $1 \mathrm{pCi} / \mathrm{gm}$ (strontium-90 level was $1.4 \mathrm{pCi} / \mathrm{gm}$ ). The radionuclide levels in the small mammal burrows and ant mounds are generally comparable or lower than the levels reported for soll in the 100 Areas as part of Westinghouse Hanford's routine monitoring program (Schmidt et al. 1992). Like the ant mounds, the limited number of burrows sampled in this study do not indicate any significant problems with small mammals exposing underground contamination. However, mammal burrowing activity is dynamic and varies through time so continued monitoring at selected waste sites might be warranted. 
WHC-EP-0620

\subsection{SUMMARY AND RECOMMENDATIONS}

\subsection{DATA EVALUATION PROBLEMS}

At the Hanford Site, the primary emphasis of the monitoring and survelllance programs and site characterization studies has been concerned with radionuclide concentrations in various media. These data-gathering efforts conducted by Westinghouse Hanford and PNL have provided information regarding radionuclide uptake in solls, plants, and animals.

Recent7y, the RCRA and CERCLA site characterization programs have dictated the need to analyze these same media for organics and other inorganic waste constituents such as heavy metals. This type of data is relatively new at the Hanford Site, and heavy metals information has been published only in the last year. According to some of the authors of these documents, questions have surfaced regarding how to quantify and/or present heavy metals information in some meaningful context.

The heavy metals data sets that are presented in this document in Appendixes $B$ through $G$ and other Hanford Site documents (e.g., Peterson and Johnson 1992 and Brandt et al. 1993) have indicated potential problems that can greatly effect how these data are interpreted and presented. Three areas of concern include analytical laboratory accuracy and precision, data validation qualifiers, and the variance observed in some of the duplicate samples.

PNL recently presented some data (Brandt et al. 1993) that indicated potential biases in analytical laboratory results. Analytical standards (pine needles and peach leaves) with known concentrations of heavy metals were sent to an analytical laboratory to serve as a quality-control check. The results of this effort indicated that many of the constituents were under or over reported by large factors. Concentrations of aluminum were underreported by $40 \%$ and iron by 25\%. Uranium was over reported two to eight times and copper was over reported by two times. This is the same analytical 1aboratory that presented the data sets in this report.

The disposition of data with a particular data validation qualifier also seems to be treated differently by authors. No meaningful guidance relating to the disposition of data and associated data validation qualifiers was found. A wide diversity of opinion exists within the scientific community about which kinds of data and associated data validation qualifiers are appropriate for statistical treatments. Often a particular data set with an associated data validation qualifier may be used or rejected based on the same reason the data was qualified in the first place. For example, in the data validation process, a set of data for heavy metals may be validated as $U^{\top}$, $\mathrm{J}^{2}$, or $\mathrm{UJ}^{3}$. If the reason is because the holding times were missed by the

$1 U$-Not detected; value reported is sample quantitation limit.

2

J=Not detected; associated value is estimated.

$3 \mathrm{U}=$ Not detected; may not accurately reflect sample quantitation limit. 
analytical laboratory and the data represents heavy metals information then the decision might be made to treat the results as real values, because metals will not decay with time. Many results were reported in this manner. The data in this report, particularly the duplicate samples, indicate that more variation occurs than would be predicted in some of the metals results.

This can make data interpretation more difficult unless large sample sizes are involved, which usually is not possible.

Obviously, potential problems are associated with heavy metals data and care must be exercised before emphasizing a given concentration value or treating the data with standard statistical treatments. Perhaps the real. value of the metals data being collected at the Hanford site will be in observing relative trends over time.

\subsection{SUMMARY}

The data presented in this report represent a substantial database that can be used for comparative purposes in future sampling efforts at the Hanford Site. This report provides data for many types of media that are not normally sampled (e.g., grass, burrows, ants, asparagus, trees). The levels reported here for metais and radionuclides are well below those levels considered to be of environmental concern. The radionuclide levels for cesium-137 and strontium-90 are generally lower than those reported by Westinghouse Hanford in their annual surveillance and monitoring reports (Schmidt et al. 1992) since 1981 from the 100 Areas, except for some elevated strontium-90 levels in mulberry trees near $100-B C$ and $100-K$ reactors.

There were some interesting trends noted with the metals data, especially the zinc concentrations in all the media. Zinc values tended to be higher than the soil threshold values for all media sampled. If true background samples were collected and analyzed for zinc and other metals in all media at the Hanford site, the results might indicate zinc levels comparable to what was reported in this study. Other metal/media combinations that were slightly elevated compared to the soil threshold values were copper in trees collected in July 1992; and antimony, cadmium, lead, and zinc in milfoll. Also, some data showed the difference in uptake between the various media that were sampled (Appendix J). For example, the lead concentrations in coyotes and raptors were obviousiy higher than the controls, which might indicate a need for further investigations. Aluminum and chromium concentrations in small mammal burrows were generally higher than for any other media.

\subsection{RECONMENDATIONS}

Data is lacking at the Hanford Site for plant and animal background values. Meaningful comparisons are difficult to make in plants and animals given the absence of Hanford Site background metal and radionuclide values. The necessary background data should be collected at the Hanford Site in plants and animals in various soil types so that ongoing and future sampling efforts can provide more meaningful interpretations of the constituents that are being analyzed and, thus, better support remedial actions and clean up measures. 
The sampling effort presented in this report is meant to serve as a general screening for pathways throughout the 100 Areas. A more intensive and site-specific effort may be required to answer specific questions at each operable unit.

\subsection{REFERENCES}

Adriano, D. C., 1986, Trace Elements in the Terrestrial Environment, SpringerVelag New York Inc.

Allen, J. N., 1980, "The Ecology and Behavior of the Long-Billed Curlew in Southeastern Washington," Wild ife Monographs, No. 73, pp. 67.

American Ornithologists Union, 1983, Checklist of North American Birds, Sixth Edition, American Ornithologists Union, Washington, D.C.

Antonio, E. J., T. M. Poston, and W. H. Rickard, 1993, Radiological survey of Shoreline Vegetation from the Hanford Reach of the Columbia River, 19901992, PNL-8797, Pacific Northwest Laboratory, Richland, Washington.

Baker, D. E., 1974, "Copper-Soil, Water Plant Relationships," Proc Fed Am Soc Exp Biol 33:1188-1193.

Beath, 0. A., H. F. Eppson, and C.S. Gilbert, 1937, "Selentum Distribution in and Seasonal Variation of Type Vegetation Occurring on Selentferous Solls," Journal Am Pharm Assoc 26:394-405.

Benson, N. R., 1966, Zinc Retention by Soils, Soll Science 101:171-179.

Beyer, W. N., R. L. Chaney and B.M. Mulhern, 1982, "Heavy metal concentrations in earthworms from soll amended with soll sewage," Journal Env. Quality $11(3): 381-385$.

Bingham, F. T., and A. L. Page, 1975, Proc Intl Conf on Heavy Metals in the Environment, 433-441, Toronto, Ontario, Canada.

Bowen, H. J. M., 1979, Environmental Chemistry of the Elements, Academic Press, New York, New York.

Brandt, C. A., C. E. Cushing, W. H. Rickard, N.A. Cadoret, R. Mazaika, and B. L. Tiller, 1993, Biological Uptake of 300-FF-5 Operable Unit Contaminants, WHC-SD-EN-TI-122, Rev. 0, West inghouse Hanford Company, Richland, Washington.

Brandt, C. A., and W. H. Rickard, Jr., 1992, Metals Concentrations in Vegetation of the 300-FF-I Operable Unit and Vicinity, WHC-SD-EN-TI-026, Rev. 0, Westinghouse Hanford Company, Richland, Washington.

Carson, B. L., and I. C. Smith, 1977, In Midwest Res Inst Tech Rep 5, pp. 386, Kansas City, Missouri. 
WHC-EP-0620

Chapman, H. D., ed., 1966, Diagnostic Criteria for Plants and Solls, Quality Printing Co., Abilene, Texas.

Chaudry, F. M., F. Hussain, and A. Rashid, 1977, "Phosphorous-Zinc Interaction in Rice," Plant Soil, 47:297-302.

Chino, M., 1981, (K. Kitagishi and I. Yamane, eds.) "Heavy metal pollution in solls of Japan," Japan Sci Soc Press, Pp. 65-80, Tokyo, Japan.

Conkl in, A. W., R. E. Elder, and W. L. Osborne, 1983, Rockwell Hanford Operations Environmental Survelllance Annual Report - Calendar Year 1982, RHO-HS-SR-82-13 P, Rockwell Hanford Operations, Richland, Washington.

Conkl in, A. W., R. E. Elder, W. L. Osborne, and J. M. Pisarcik, 1982, Rockwell Hanford Operations Environmental Survelllance Annual Report - Calendar Year 1981, RHO-HS-SR-82-4 P, Rockwell Hanford Operations, Richland, Washington.

Cushing, C. E., 1979, "Trace Elements in a Columbia River Food Web," Northwest Sclence, Vol. 53, No.2, Pp. 118-125.

Cushing, C. E., 1993, Aquatic Studies at the 100-HR-3 and 100-NR-1 Operable Units, PNL-8584, Pacific Northwest Laboratory, Richland, Washington.

Davis, R.D., P.H.T. Beckett, and E. Wollan, 1978, "Critical Levels of 20 Potentially Toxic Elements in Young Spring Barley," Plant Soil, 49:395408.

DOE-RL, 1992a, Remedial Investigations/Feasibility Study Work Plan for the 100-BC-5 Operable Unit, Hanford Site, Richland, Washington, DOE/RL-90-08, U.S. Department of Energy Richland Operations Office, Richland, Washington.

DOE-RL, 1992b, Sampling and Analysis of 100 Area Springs, DOE/RL-92-12, Rev. 1, U.S. Department of Energy Richland Operations Office, Richland, Washington.

DOE-RL, 1993, Hanford Site Soll Background: Part 1, Soll Background for Nonradioactive Analytes - Volumes 1 and 2, DOE/RL-92-24, U.S. Department of Energy Richland Operations Office, Richland, Washington.

Ecology, 1992, Model Toxic Control Act, Washington State Department of Ecology, 01ympia, Washington.

Endangered Species Act of 1973, as amended, 16 USC 1531, et seq.

Ennor, H. R., 1991, Birds of the Tri-Cities and Vicinity, Lower Columbia Basin Audubon Society, Richland, Washington.

ERDA, 1975, Final Environmental Impact Statement: Waste Management Operations, Hanford Reservation, Richland, Washington, ERDA-1538, 2 Vols., U.S. Energy Research and Development Administration-Richland Operations Office, Richland, Washington. 
Fickelsen, D. H., R. E. Fitzner, R. H. Saver, and J. L. Warren, 1980, Wildilfe Usage, Threatened and Endangered Species and Habitat Studies of the Hanford Reach, Columbia River, Washington, Pacific Northwest Laboratory, Richl and, Washington.

Fitzner, J. N., 1978, The Ecology and Behavior of the Long-Billed Curlew (Numenius americanus) in Southeastern Washington, Ph.D. Thesis, Washington State University, Pullman, Washington.

Fitzner, J. N., K. A. Gano, W. H. Rickard, and L. E. Rogers, 1979, Characterization of the hanford 300 Area Burial Grounds: Task IVBiological Transport, PNL-2774, Pacific Northwest Laboratory, Richland, Washington.

Fitzner, J. N., and R. H. Gray, 1991, "The Status, Distribution and Ecology of Wildlife on the U.S. DOE Hanford Site: A Historic Overview of Research Activities," Environmental Monitoring and Assessment, 18:173-202.

Fitzner, R. E. (Pacific Northwest Laboratory) and S. G. Welss (Westinghouse Hanford Company), 1992, Bald Eagle Site Management Plan for the Hanford Site, South-Central Washington, WHC-EP-0510, Westinghouse Hanford Company, Richland, Washington.

Fitzner, R. E. (Pacific Northwest Laboratory), S. G. Weiss and J. A. Stegen (West inghouse Hanford), 1992, Blological Assessment for Threatened and Endangered Wildlife Species, WHC-EP-0513, Westinghouse Hanford Company, Richland, Washington.

Fitzner, R. E., W. H. Rickard, L. L. Cadwell, and L. E. Rogers, 1981, Raptors of the Hanford Site and Nearby Areas of Southcentral Washington, PNL-3212, Pacific Northwest Laboratory, Richland, Washington.

Fitzner, R. E. and R. L. Newel1, 1989, "Ferruginous Hawk Nesting on the U.S. DOE Hanford Site: A Recent Invasion Following Introduction of Transmission Lines," Issues and Technology in the Management of Impacted Western Wildlife, Proceedings of a National Sympostum, Thorne Ecological Institute, Glenwood Springs, Colorado.

Friberg, L., M. Piscator, and G. Norberg, 1971, Cadmium in the Environment, CRC Press, Cleveland, Ohto.

Gal1, 0. E., and R.M. Barnette, 1940, "Toxic Limits of Replaceable Zinc to Corn and Cowpeas Grown on Three Florida Solls," Journal American Society of Agronomy, 32:23-32.

Greager, E. M., 1981, Biotransport of Radionuclides in the 100 Areas, UNI-1888, UNC Nuclear Industries, Richland, Washington.

Haghirf, F., 1973, "Cadmium Uptake by Plants," Journal Environmental Quality, 2:93-96.

Ha11, J. A, 1989, "Aspects of Forster's Tern (Sterna forsteri) Reproduction on Cobblestone Islands in Southcentral Washington," Northwest

Science, 63(3):90-95. 
Hitchcock, C. L. and A. Chronquist, 1973, Flora of the Pacific Northwest, University of Washington Press, Seattle, Washington.

Hoover, J. D. and T. Legore, 1991, Characterization and Use of Soll and Groundwater Background for the Hanford Site, WHC-MR-0246, Westinghouse Hanford Company, Richland, Washington.

Hoy, T., N. Berg, J. T. Fyles, G. D. Delaney, D. McMurdo, and P. W. Ransom, 1985, Tratbound Base Metal Deposits in Southwestern British Columbia, in Field Guides to Geology and Mineral Deposits in the Southern Canadian Condillera, D. Templeman-Klutt (ed), Geological Society Assoc of Canada, Vancouver, British Columbia

Johnson, V. G., 1979, Decline of Radioactivity in the Columbia River and Estuary: Rates and Mechanisms, PH.D. Thesis, Oregon State University, Corvalits, Oregon, PP. 126.

Johnson, V. G., 1991, Contaminant Storage and Mixing in Bed Sediment of the Lower Columbia River and Estuary, Supplement to EOS, Transactions, American Geophysical Union, Vol. 72, No. 44.

Khalld, B. Y., and J. Tinsley, 1980, "Some Effects of Nickel Toxicity on Rye Grass," Plant Soll, 55:139-144.

Kubota, J., 1983, Copper Status of United States Solls and Forage Plants, Agronomy Journat, 75:913-918.

Landeen, D. S., 1992, Description of Work for 100 Areas Operable Unit Ecological Investigations, WHC-SD-EN-AP-090, Westinghouse Hanford Company, Richland, Washington

Landeen, D. S., A. R. Johnson, and R. M. Mitche11, 1992, Status of Birds at the Hanford Site in Southeastern Washington, WHC-EP-0402, Rev. 1, Westinghouse Hanford Company, Richland, Washington.

Landeen, D. S., and R. M. Mitche11, 1981, Intrusion of Radioactive Waste Burial Sites by the Great Basin Pocket Mouse (Perognathus parvus), RHO-SA-211, Rockwell Hanford Operations, Richiand, Washington.

Landeen, D. S., and R. M. Mitchel1, 1982, The Role of Burrowing Activities of the Great Basin Pocket Mouse (Perognathus parvus) in the Dispersal of Radionuclides on Decommissioned Pond, RHO-HS-SA-10 P, Rockwell Hanford Operations, Richland, Washington.

Landeen, D. S., and R. M. Mitchell, 1986, "Radlonuclide Uptake by Trees at a Radwaste Pond in Washington State," Health Physics, Vol. 50, No. 6, pp. 769-774.

Landeen, D. S., and M. R. Sackschewsky, 1992, Fiscal Year 1991100 Areas CERCLA ECological Inverstigations, WHC-EP-0488, Westinghouse Hanford Company, Richland, Washington. 
Likens, G. E. and F. H. Bormann, 1970, In Chenical Analyses of Plant Tissue from the Hubbard Brook Ecosystem in New Hampshire, Yale School Forestry Bull. no. 79.

Lozano, F. C., and I. K. Morrison, 1981, "Oisruption of Hardwood Nutrition by Sulfur-Dioxide, Nickel and Copper Air Pollution Near Sudbury, Canada," Journal Environmental Quality, 10:198-204.

Maclean, A. J., 1974, "Effects of Sol1 Properties and Amendments on Avallability of Zinc in Solls," Canadian Journal of Soll Science, 54:369378.

Marten, G. C., and P. B. Hammond, 1966, "Lead Uptake by Bromegrass From Contaminated Solls," Agronomy Journal, 58:553-554.

Mashred/Cement Mason, T. G., and E. Ph1111s, 1937, "A New Method of Control for Insect Pests of the Cotton Plant," Emplre Cotton Grow Rev, 14:308309.

Mastrogfuseppe, J. D. and S. J. G111, 1983, "Steppe by Step: Understanding Priest Raplds Plants," Douglasia Occastonal Papers, Washington Native Plant Society Vol. 1, Marion Ownbey Herbarfum, Washington State University, Pulliman. Washington.

Maxfleld, D., J. M. Rodriquez, M. Buettner, J. Davis, L. Forbes, R. Kovacs, W. Russe1, L. Schultz, R. Smith, J. Stanton, and C. W. Wal, 1974, "Heavy Metal Content in the Sediments of the Southern Part of the Coeur d'Alene Lake, Environmental Pollution, Vol. 6, Pp. 263-266.

McGahan, J., 1967, "Quantifled estimates of predation by a golden eagle population," Journal Wildiffe Mgnt, 31:496:501.

Melton, J. R., B. G. Ell1s, and E.C. Doll, 1970, "Zinc, Phosphorous, and Lime Interactions With Yteid and Zinc Uptake by Phaseolus vulgaris," Soll science Soc. Am Proc, 34:91-93.

Miller, W. E., J. L. Greene, T. Shiroyama, and E. Merwin, 1975, "The Use of the Algal Assay Method to Determine Effects of Waste Discharges in the Spokane River System," in Proc.: Biostimulation Nutrient Assessment Workshop, Oct. 1973, EPA 660/3-75-034, U.S. Environmental Protection Agency, Washington, D.C.

Miller, J. E.. J. J. Hassett, and D.E. Koeppe, 1977, "Interactions of Lead and Cadmium on Metal Uptake and Growth of Corn Plants," Journal of Environmental Quality, 6:18-20.

Mitchell, R. L., 1993, "Cobalt and Nickel in Solls and Plants," Soll Science, $60: 63-70$.

NAS, 1973, Manganese, Pp. 191, National Academy of Sciences, Washington, D.C.

Ohk1, K., 1975, "Lower and Upper Critical Zinc Levels in Relation to Cotton Growth and Development," Physiol Plant, 35:96-100. 
Peterson, P. J., 1975, In Proc Int I Conf on Heavy Metals in the Environment, 39-54, Toronto, Ontarfo, Canada.

Peterson, R. E., and M. P. Connelly, 1992, Characterization of a Chroniun Plume in Groundwater Along the Columbla River Shorel the, Hanford site, Washington, WHC-SA-1674-VA, Westinghouse Hanford Company, Richland, Washington.

Peterson, R. E., and V. G. Johnson, 1992, Riverbank Seepage of Groundwater Along the 100 Areas Shoreline, Hanford Site, WHC-EP-0609, West inghouse Hanford Company, Richland, Washington.

Poole, L. D., 1992, Reproductfve Success and Nesting Habltat of Loggerhead Shrikes in Shrubsteppe Communities, Masters Thes1s, Washington State University, Pulliman, Washington.

Porter, S. D., and C. D. Jorgensen, 1988, "Longevity of Harvester Ant Colonies in Southern Idaho," Journal of Range Management, 41(2):104-107.

Pratt, P. F., 1966, (H. D. Chapman, ed.) Diagnostic criteria for plants and solis, Pp. 136-141, Quality Printing Co., Inc, Abllene, Texas.

Reldel, S. P., and K. A. Lindsey, and K. R. Fecht, 1992, Field Trip Guide to the Hanford Site, WHC-MR-0391. Westinghouse Hanford Company, Richland, Washington.

Rember, W. C., S. F. Bender, V. E. Chamberlain, M. L. Hoffman, and K. F. Sprendke, 1991, "An Investigation of Mine Tallings and Heavy Metals in the Coeur d'Alene River Lateral Lakes, Kootenal County, Idaho," Supplement to EOS, Transactions, American Geophysical Union, Vol. 72, No. 44.

Rickard, W. H., R. E. Fitzner, and C. A. Brandt, 1990, Biota of the 300-FF-1 Operable Unit, EMO-1016, Environmental Management Operations, Richland, Washington.

Rickard, W. H., J. D. Hedlund, and R. G. Schreckhtse, 1974, Mammals of the Hanford Reservation in Relation to Management of Radioactive Waste, BNWL-1877, Battelle Paciflc Northwest Laboratories, Richland, Washington.

Rickard, W. H., J. D. Hedlund, and R. E. Fitzner, 1978, "Rejecta Cast from Heron Nests as an Indicator of Food Chain Contamination," The Auk, $95(2): 425-427$.

Rickard, W. H., and K. R. Price, 1989, "Uptake of Tritiated Groundwater by B1 ack Locust Trees," Northwest Science, Vol. 63, No. 3, Pp. 87-89.

Robbins, J. M., 1978, Weathering and Microbial Activity in Sulfidic Mine Tallings With Implications in Reclamation. Thesis, Department of Soll Sclence, University of British Columbia.

Rogers, L. E. and W. H. Rickard, 1977, Ecology of the 200 Area Plateau Waste Management Environs: A Status Report, PNL-2253, Pacific Northwest Laboratory, Richland, Washington. 
Sackschewsky, M. R., 1992, Blological Assessment for Rare and Endangered Plant Species - Related to CERCLA Cheracterization Activities, WHC-EP-0526, Westinghouse Hanford Company, Richland, Washington.

Sackschewsky, M. R., D. S. Landeen, J. L. Downs, W. H. Rickard, G. I. Baird, 1992, Vascular Plants of the Hanford Stte, WHC-EP-0554, Westinghouse Hanford Company, Richland, Washington.

Sackschewsky, M. R. and D. S. Landeen, 1992, Fiscal Year 1991100 Areas CERCLA Ecological Investigations, WHC-EP-0448, Westinghouse Hanford Company, Richland, Washingtion.

Sauer, R. H. and J. E. Leder, 1985, "The Status of Persistent Sepal Yellowcress in Washington," Northwest Sctence 59:198-203.

Scanion, P. F., 1979, "Ecological tmplications of heavy motal contamination of roadside habitats," Proc. S.E. Assoc. Fish and Wildlife Agenc., 33:315330.

Schmidt, J. W. A. R. Johnsnn, S. M. Mckinney, C. J. Perkins and C. R. Wabb, 1992, Westinghouse Hanford Company Environmental survellliance Annual Report Calendar Year 1991, Westinghouse Hanford Company, Richland, Washington.

Sllker, W. B., 1964, "Variations in Elemental Concentrations in the Columbia River," Limnology and Oceanography, Vol. 9, No. 4, Pp. 540-545.

St. John, H. and G. N. Jones, 1928, An Annotated Catalog of the Vascular Plants of Benton County. Washington, Contributions of the Department of Botany of the State College of Washington. Number 9, pp. 73-93.

Smith, W. H., 1973, "Motal Contamination of Urban Woody Plants, "Environmental Sctence Technology, 7:631-636.

Soldat, J. K., K. R. Price, and W. H. R1ckard, 1990, Mothodology Used to Compute Maximum Potential Doses from Ingestion of Edible plants and Wildife Found on the Hanford Site, PNL-7539, Pacific Northwest Laboratory, Richland, Washington.

Soltanpour, P. N. and S. M. Workman, 1980, "Use of NH, HCO - DTPA Soll Test to Assess Avallabllity and Toxicity of Selentum to Alfalfa Plants," Commun Soll Sci plant And, 11:1147-1156.

Stebbins, R. C., 1985, Western Reptiles and Amphibians, Houghton Miffi in Company, Boston, Massachusetts.

Stone, 1989, Preliminary Operable Units Designation Project, WHC-EP-0216, West inghouse Hanford Company, Richland, Washington.

Talmage, S. S., and B. T. Walton, 1991, "Small mammals as monitors of environmentai contaminants," Rev. Environ. Contam. Toxicol., 119:47-145. 
Thelde, M.E. 1992, Small Mammals and Harvester Ants in the 300-FF-1 Operable Unit, WHC-SD-EN-TI-028, Rev. 0, Westinghouse Hanford Company, Richland, Washington.

THller, B. L., and T. M. Poston, 1992, Uraniun Concentrations in Asparagus, PNL-8068, Pactfic Northwest Laboratory, Richland, Washington.

Tyler, G., 1972, "Heavy Metals may Pollute Nature, may Reduce Productivity," Ambio, 1:52-59.

U.S. Fish and Wildlife Service, "Endangered and Threatened Wildilfe and Plants," Title 50, Code of Federal Regulations, Part 17, as amended, U.S. Department of the Interior, Washington, D.C.

Wallace, A., R. T. Mueller, and G. V. Alexander, 1978, "Influence of Phosphorous on ZInc, Iron, Manganese, and Copper Uptake by Plants, "Soll science, 126:336-341.

Walsh, T., and G. A. Fleming, 1952, IntI soc Soll Sct Trans, Comm II and IV, 178-183.

Walsh, L. M., H. H. Erhardt, and H.D. Selbel, 1972, "Copper Toxictiy in Snapbeans," Journal of Environmental Quality, 1:197-200.

Washington Department of W11d11fe, June 1991, Spectes of Concern in Washington. Washington State Department of Wildiffe, 01 ympla, Washington.

Washington Natural Heritage Program, 1990, Endangered, Threatened and Sensitive Vascular Plants of Washington, Department of Natural Resources, Olympla, Washington.

Weiss, S. G., and R. M. Mitchell, 1992, A Synthests of Ecological Date from the 100 Areas of the Hanford Site, WHC-EP-0601, Westinghouse Hanford Company, Richland, Washington

WHC, 1991a, Ecological Data Compllations Investigations status Report for 100 Area Operable Units, WHC-MR-0272, West inghouse Hanford Company, Richland, Washington.

WHC, 1991b, Environmental Investigations and site Characterization Manual, WHC-CM-7-7, Westinghouse Hanford Company, Richland, Washington.

Wheeler, G. L., and G. L. Rolfe, 1979, Environmental Pollution, 18:265-274.

Wildung, R. E., D. A. Cataldo, and T. R. Garland, 1986, Fate of Heavy Metals and Heavy Motal Complexes in Solls and Plants, Paclfle Northwest Laboratory, Richland, Washington.

Williams, C. H. and D. J. David, 1977, "Some Effects of Distribution of Cadmium and Phosphate in Root Zone on Cadmium Uptake of Plants, "Aust Journal Soll Research, 15:59-68. 
WHC-EP-0620

Woodruff, R. K., R. W. Hanf, and R. E. Lundreng, 1993, Hanford site Environmental Report for Calendar Year 1992, PNL-8682, Paciftc Northwest Laboratory, Richland, Washington.

Young, H. E. and V. P. Guinn, 1966, "Chemical Elements in Complete Mature Trees of Seven Spectes in Maine," Tappl, 49:190-197.

\subsection{DIBLIOCRAPHY}

Arnott, R. H. and R. L. Jacques, 1981, Simon and Schuster's Guide to Insects, Simon and Schuster Inc., Now York, Now York.

Books, G. G., 1985, "Avian Interaction with Mid-Columbla River Water Level Fluctuations," Northwest Sctence, Vol. 59, No. 4.

Borror, D. J. and R. L. White, 1970, A Field Guide to Insects in Americe North of Mexico, Peterson Fleld Gulde Serles, Houghton Miffi in Company, Boston, Massachusetts.

Borror, D. J., D. M. Dolong, and C. A. Triplohorn, 1976, An Introduction to the Study of Insects. Fourth Edition, Holt, Rinchart and Winston.

Burt, W. H. and R. P. Grossenhelder, 1980, A Field Guide to the Mamals of North America. Third Edition, Houghton Mifflin Company, Boston, Massachusetts.

Daubenmire, F. R., 1970, Steppe Vegetation of Washington, Washington Agricultural Experiment Station Technical Bulletin 62, Washington Agricultural Experiment Station, Pullman, Washington.

Fickeisen, D. H., R. E. Fitzner, R. H. Sauer, and J. L. Warren, 1980, Wtldlife Usage, Threatened and Endengered Spectes and Habltat Studies of the Hanford Reach, BNW 2311104244, Battelle Pactfic Northwest Laboratorles, Richland, Washington.

Fickelsen, D. H., R. E. Fitzner, R. H. Saver, and J. L. Warran, 1980, WtIdlife Usage, Threatened and Endangered Species and Habitat studies of the Hanford Reach, Columbla River, Washington, Paciftc Northwest Laboratory, Richland, Washington.

Fitzner, R. E. and K. R. Price, 1973, The Use of Hanford Waste Ponds by Waterfowl and Other Birds, BNWL-1738, Battelle Pacific Northwest Laboratortes, Richland, Washington.

Fitzner, R. E. and W. H. Rickard, 1975, Avifaune of Waste Ponds ERDA Hanford Reservation Benton County, Washington, BNWL-1885, Battelle Paciflc Northwest Laboratories, Richland, Washington.

Hanson, W. C., 1958, "Arctic Tern and Black Brant in Eastern Washington," Murrelet, 39(1):12. 
WHC-EP-0620

Hanson, W. C., 1969, "First Slght Records of Blue Geese in Washington," Nurrelet, $50(2): 24$.

Landeen, D. S., 1992, Description of Work for 100 Areas Operable Unit Ecological Investigations, WHC-SD-EN-AP-090, Westinghouse Hanford Company, Richland, Washington

Morlarty, F., 1988, Ecotoxicology the study of Pollutants in Ecosystems, Acadentc Press, San Diego, Callfornia.

National Geographic Soctety, 1989, Field Guide to the Birds of North America, Second Edition, Washington, D.C.

Robbins, C. S., B. Brun, and H. S. 21m, 1966, Birds of North America, Golden Press, Now York, Now York.

Rotenberry, J. T., R. E. Fitzner, and W. H. Rickard, 1979, "Structure:

Differences in Mechantsms Regulating Diversity," The Auk 96:499-505.

Sackschewsky, M. R., 1992, Blological Assessment for Rare and Endangered Plant Species Related to CERCLA Characterization ACtivities, WHC-EP-0526, West Inghouse Hanford Company, Richland, Washington

Sackschewsky, M. R., D.S. Landeen, G. I. Baird, W. H. Rickard, and J. L. Downs, 1992, Vascular Plants of the Hanford Site, WHC-EP-0554, Westinghouse Hanford Company, Richland, Washington.

Schuler, C. A., W. H. Rickard, and G. A. Sargeant, 1988, Bird Associations with Shrubsteppe Plant Communities ot the Proposed Reference Repository Located in Southeastern Washington, PNL-6493, Pactflc Northwest Laboratory, Richland, Washington.

Welss, S. G., and R. M. Mitchell, 1992, A Synthesis of Ecological Data from the 100 Areas of the Hanford SIte, WHC-EP-0601. Westinghouse Hanford Company, Richland, Washington

WHC, 1991a, Ecological Data Compllation Investigations Status Report for 100 Areas Operable Units, WHC-MR-0272, Westinghouse Hanford Company, Richland, Washington. 
WHC-EP-0620

APPENDIX A

SAMPLE LOCATION MAPS FOR ALL MEDIA 
WHC-EP-0620

This page intentionally left blank. 


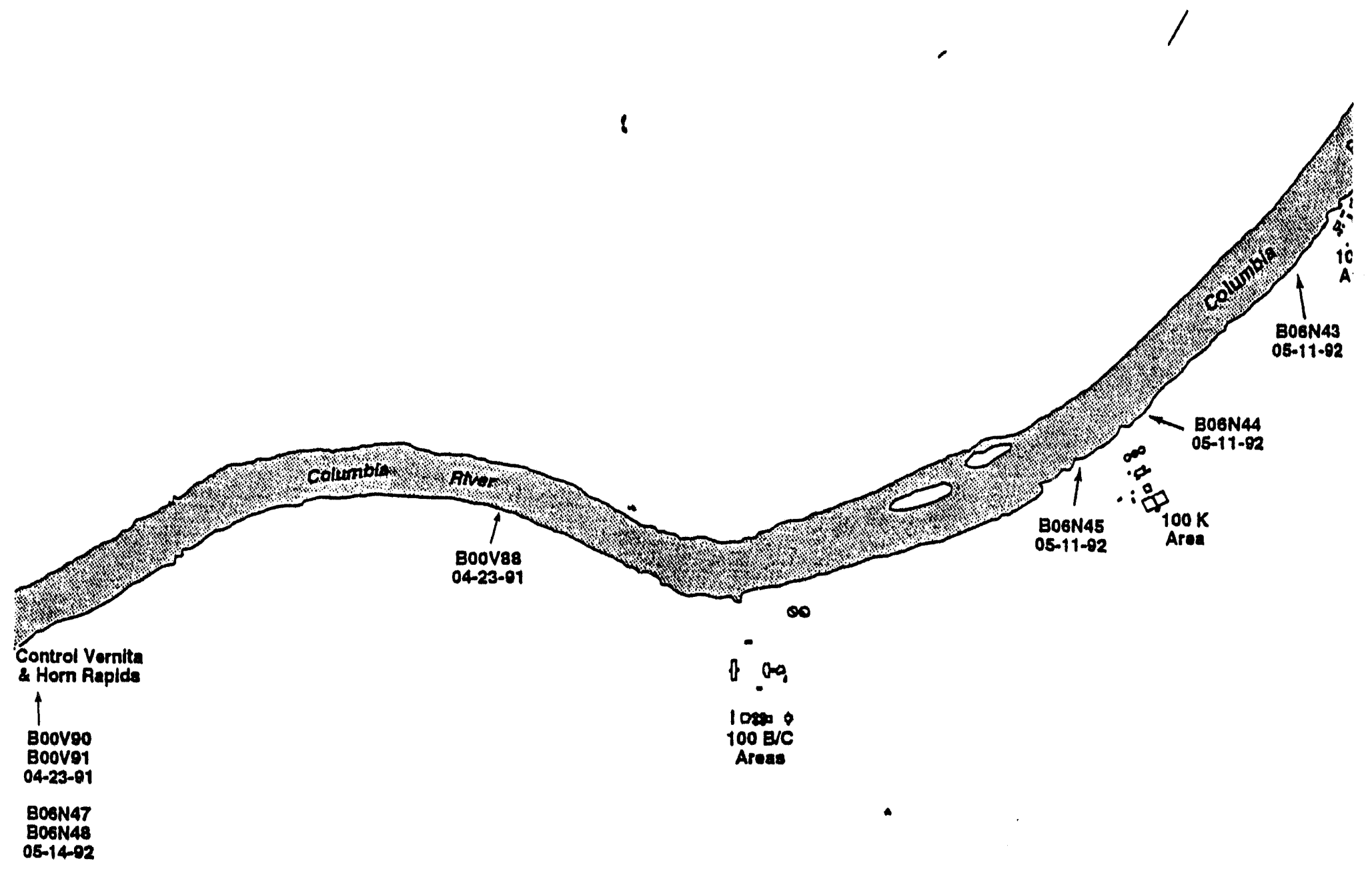


WHC-EP-0620

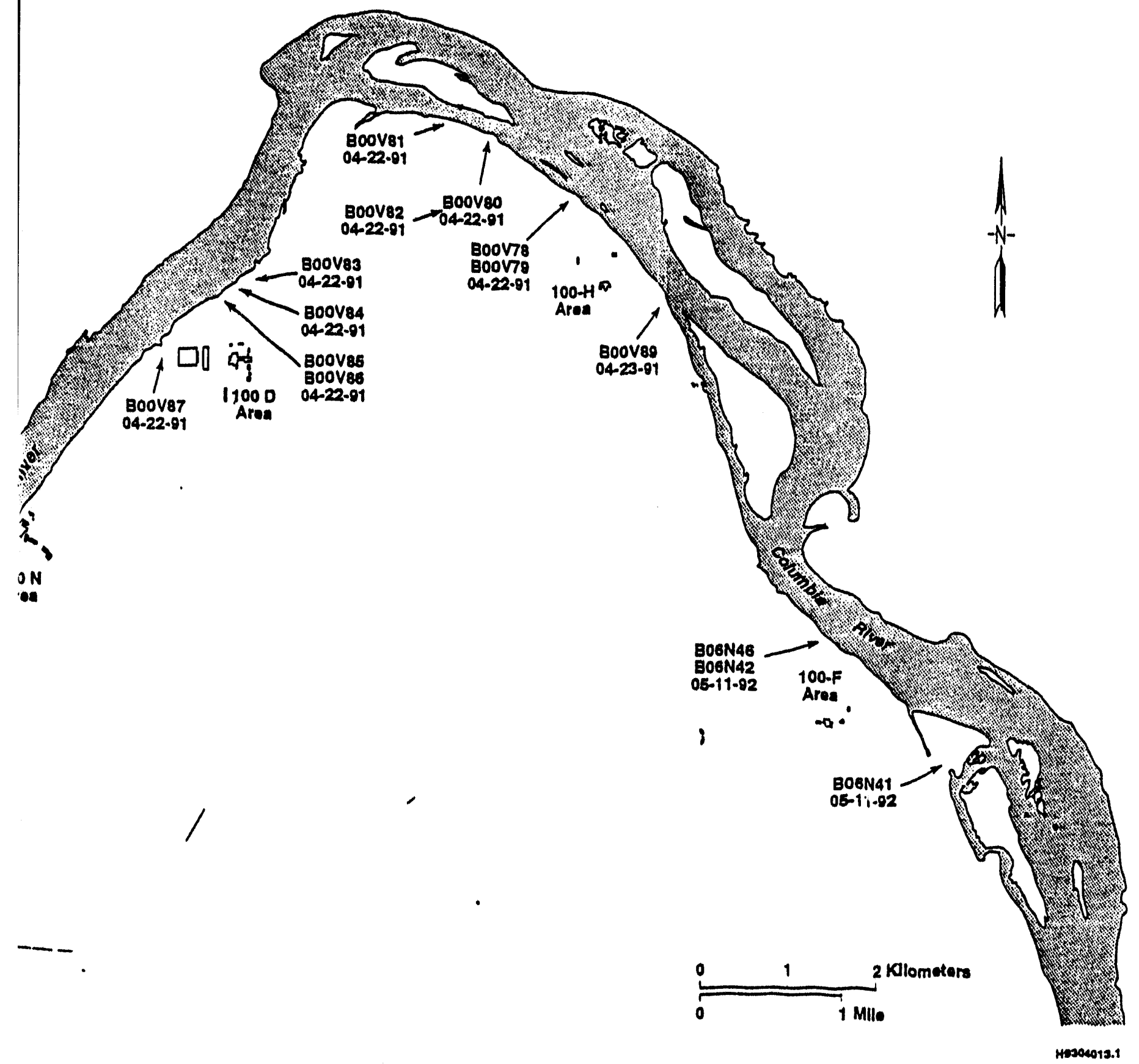

Figure A-1. Sample Locations and Identification Numbers for Asparagus Collected in 1991 and 1992. 


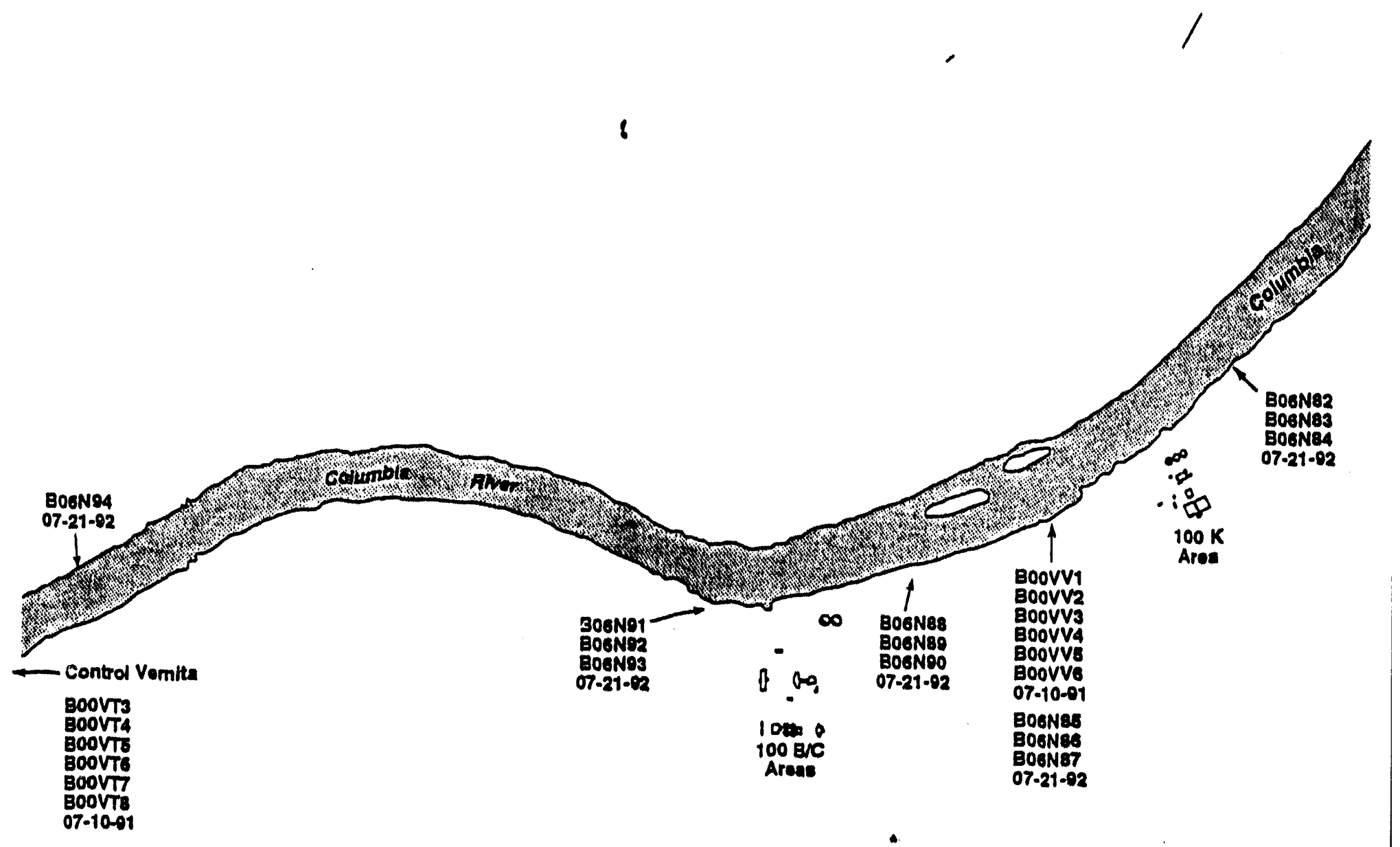




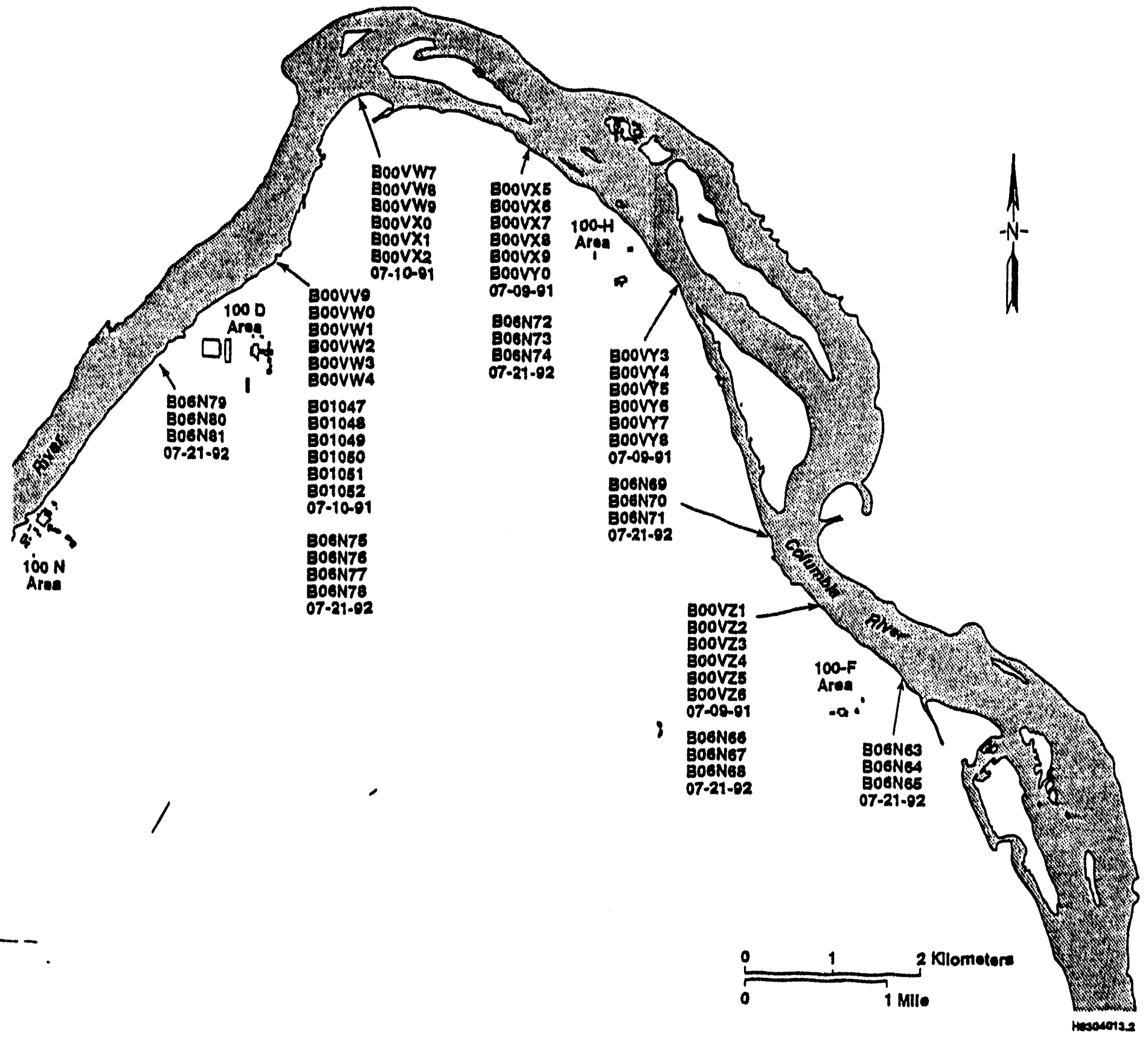

Figure A-2. Sample Locations and Identification Numbers for Reed Canarygrass Collected in 1991 and 1992. 


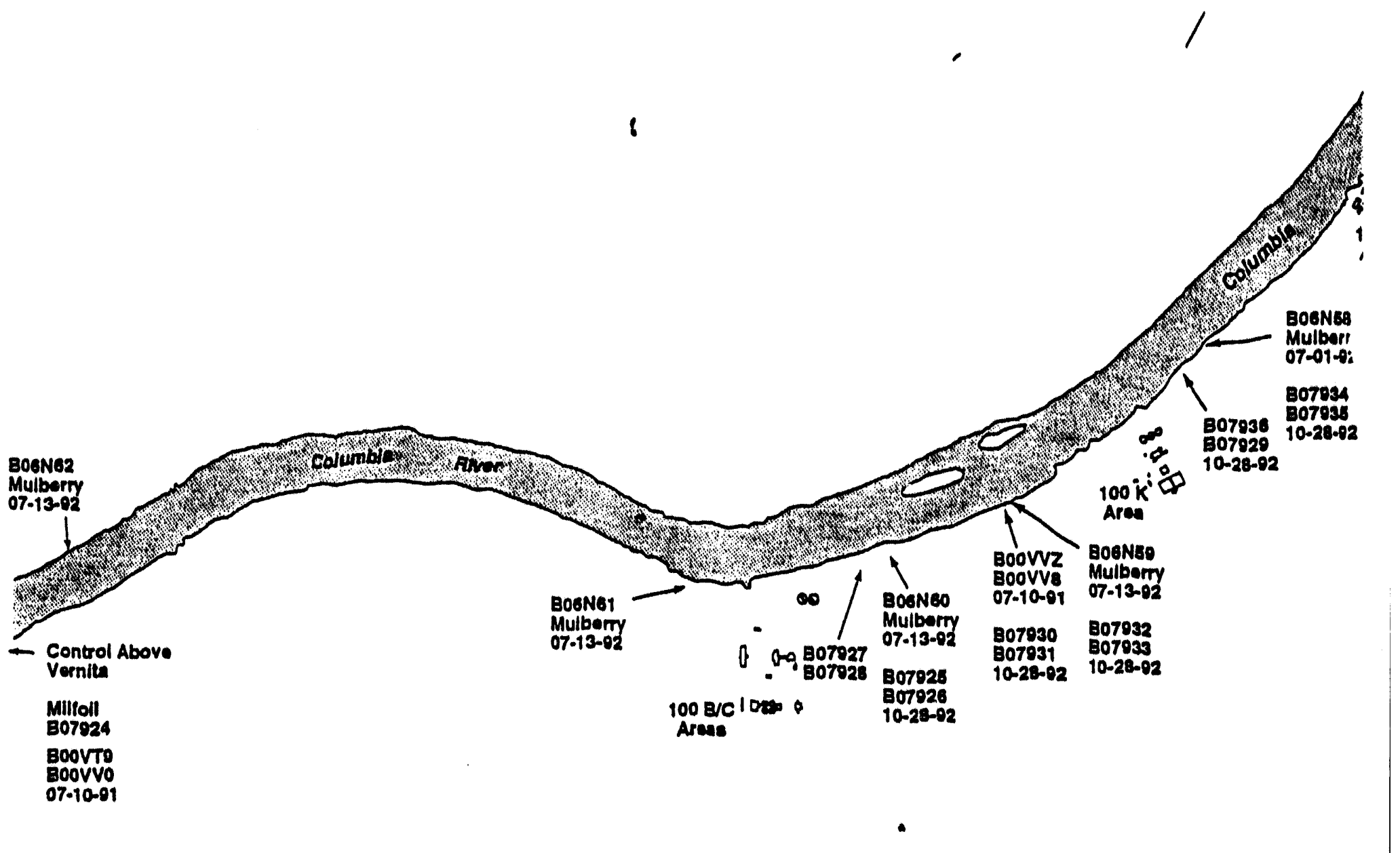




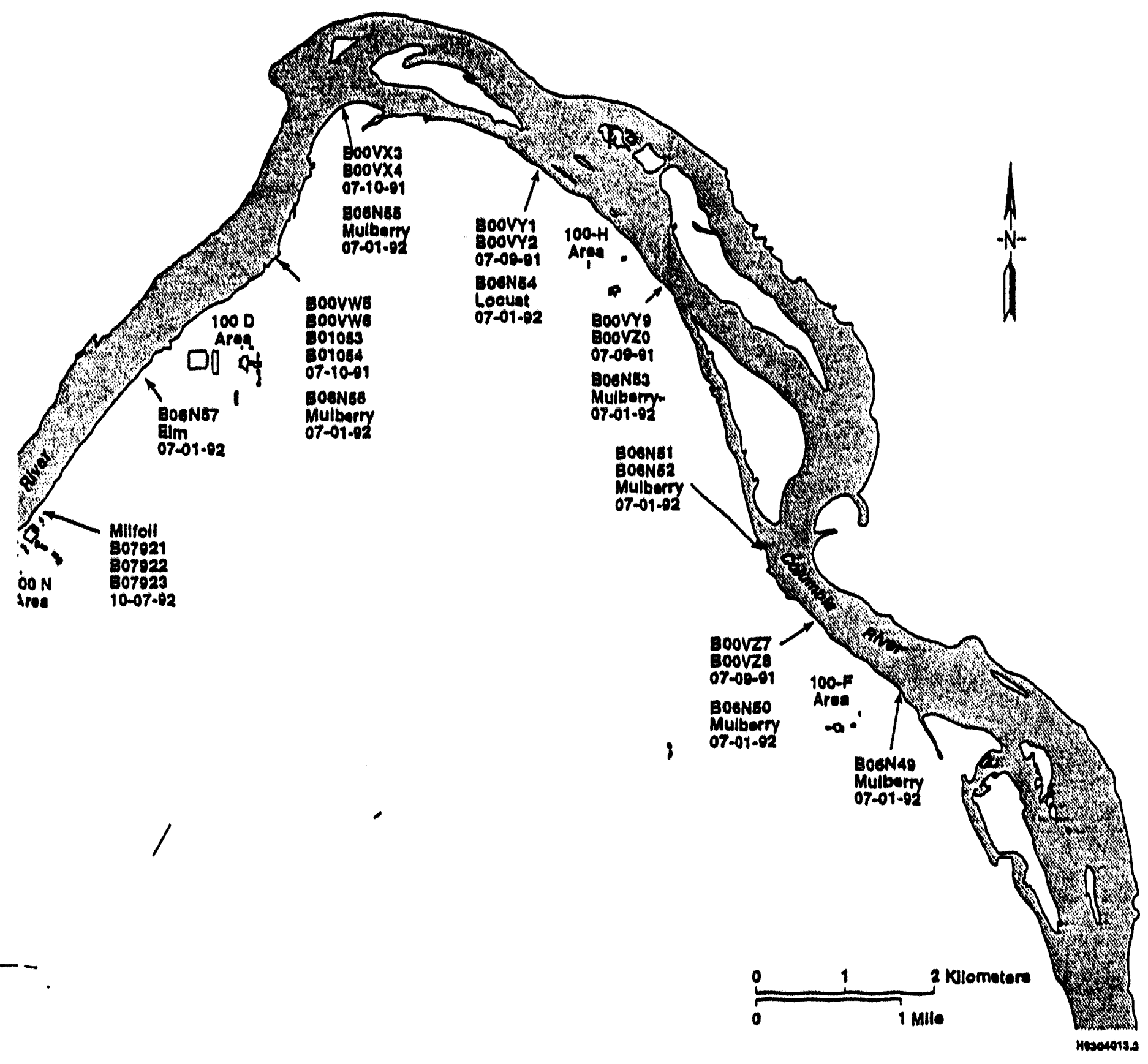

- Figure A-3. Sample Locations and Identification Numbers for Trees and Milfoil Collected in 1991 and 1992. 


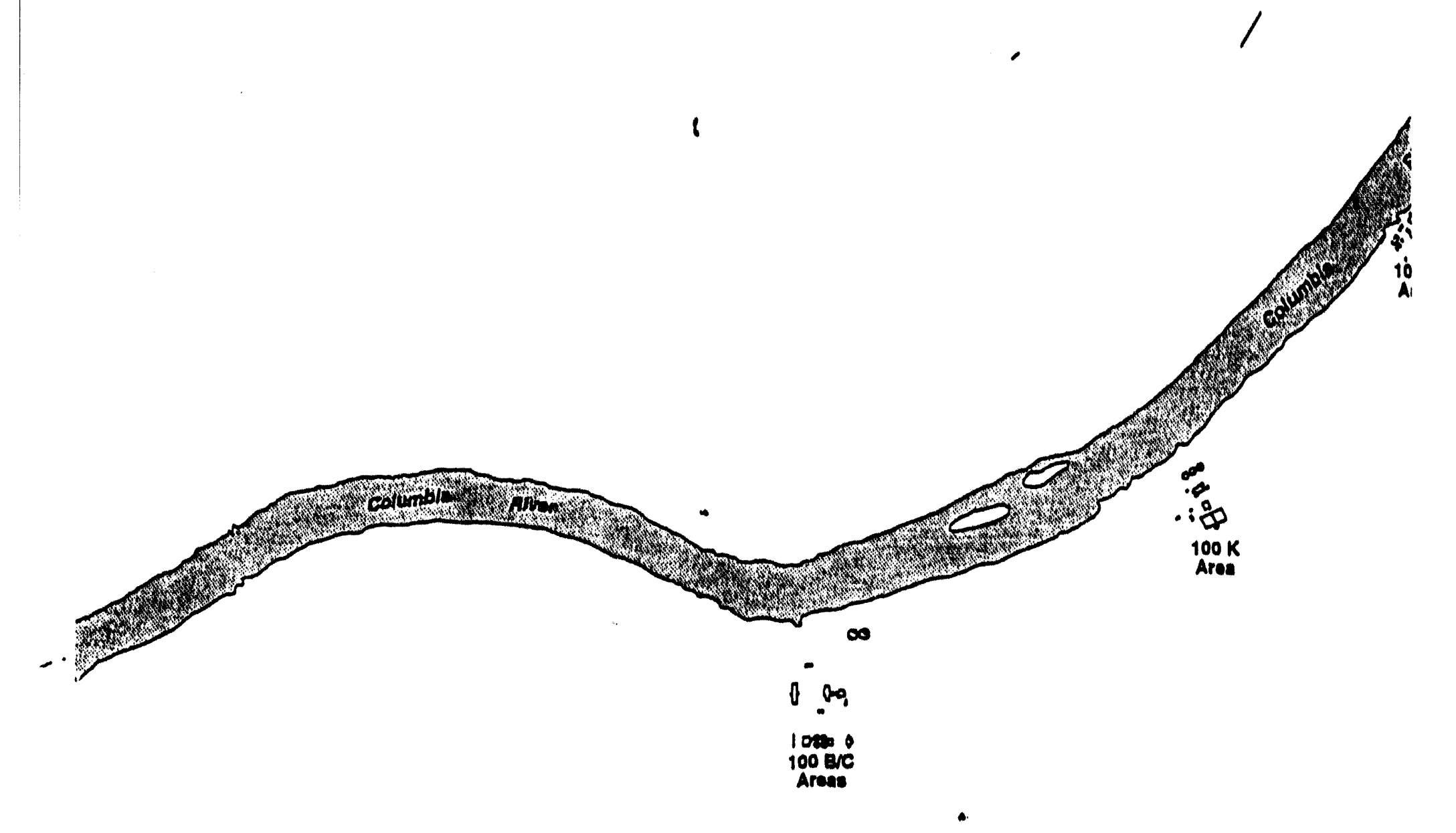




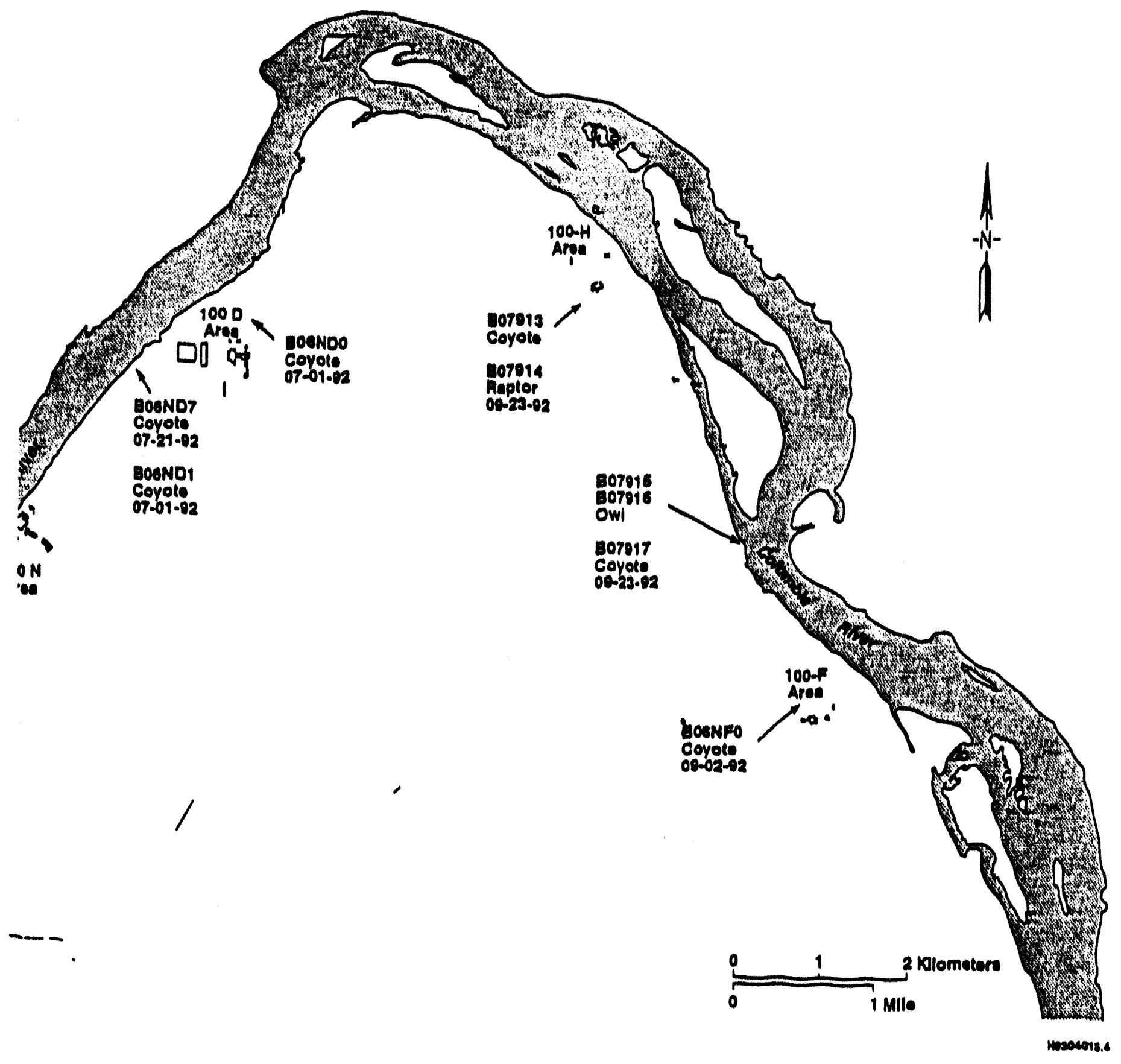

Figure A-4. Sample Locations and identification Numbers for Raptor Pellets and Coyote Scat Collected in 1992. 


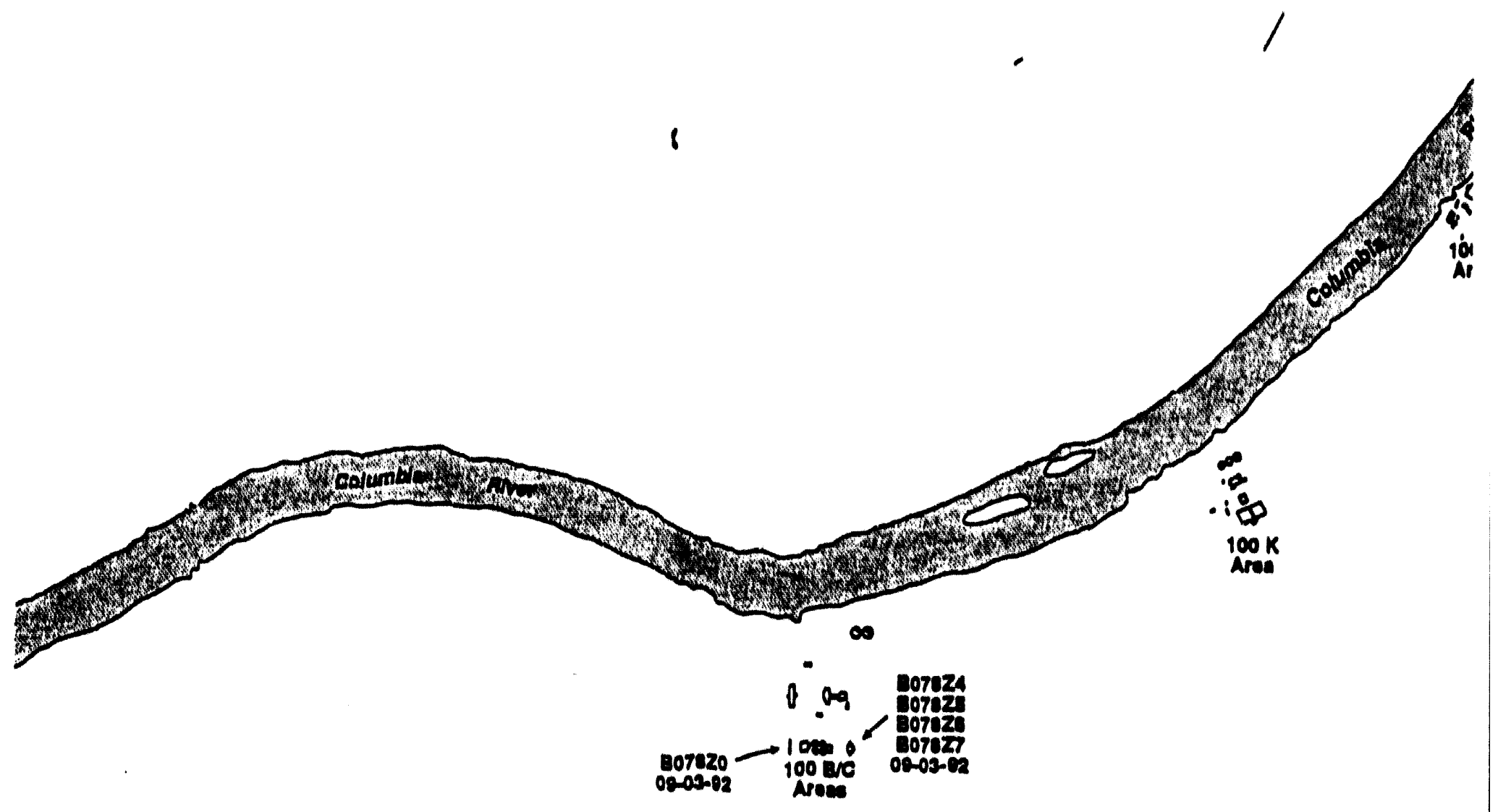

. 


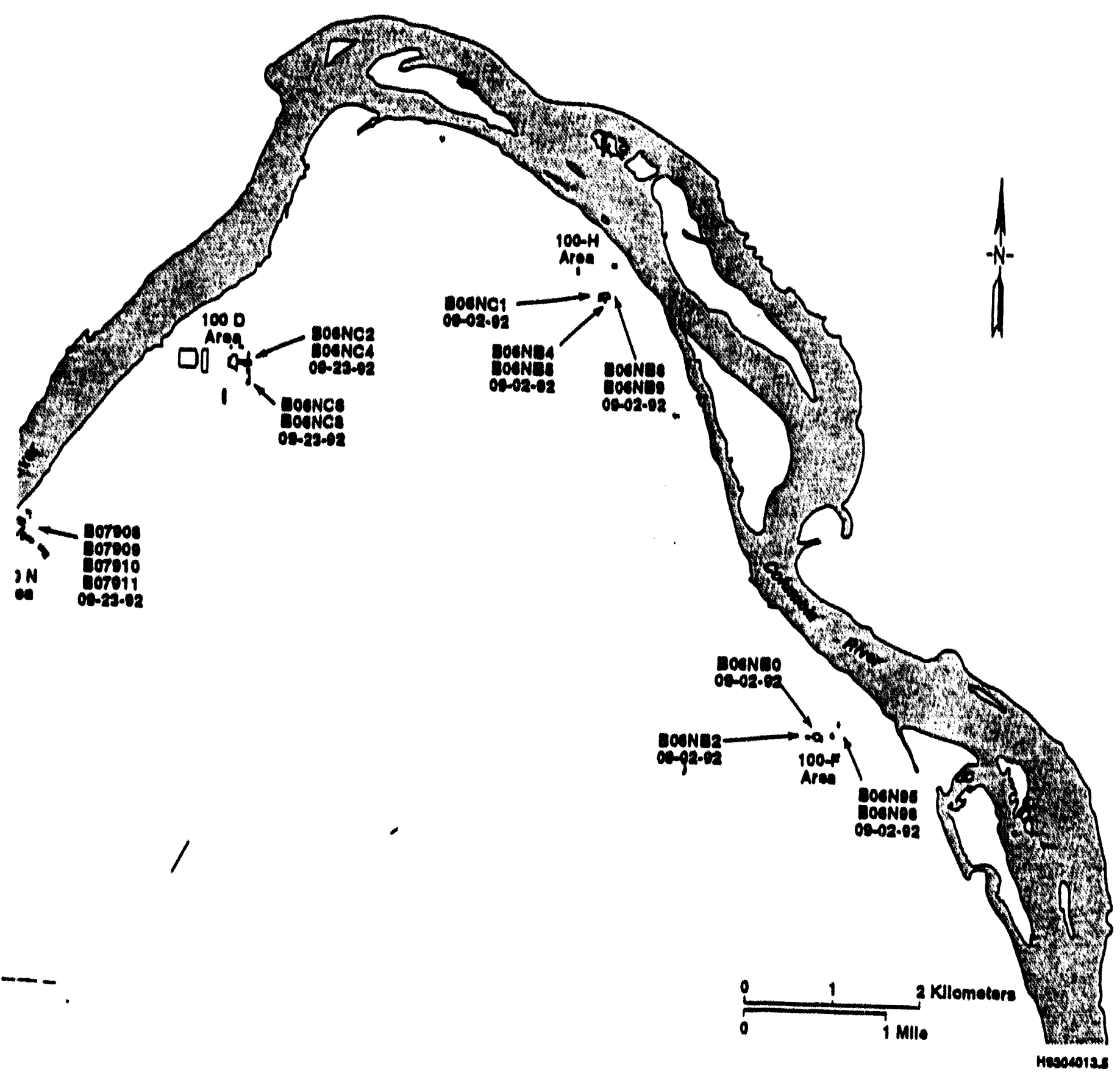

Figure A-5. Sample Locations and Identification Numbers for Ant Mounds Collected in 1992. 


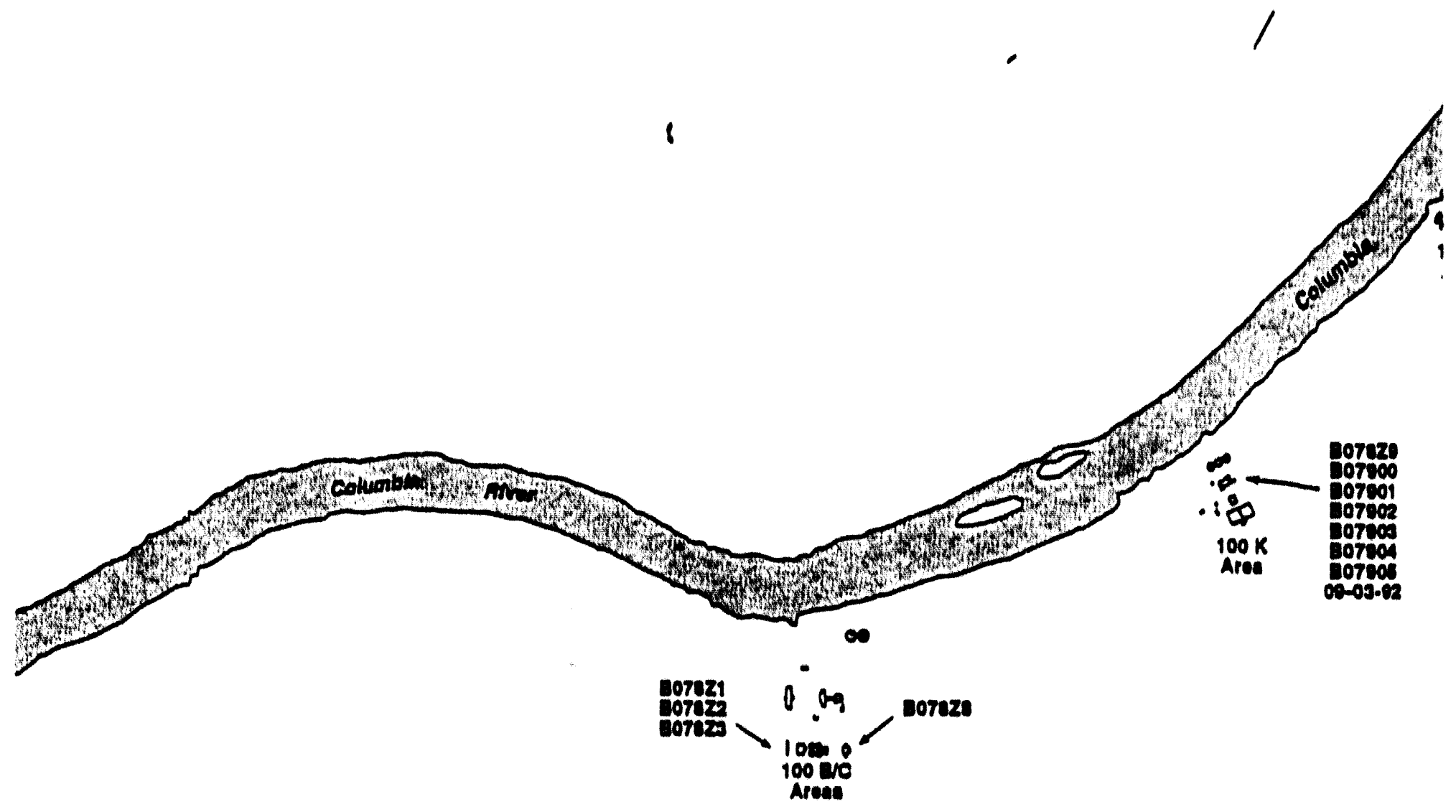




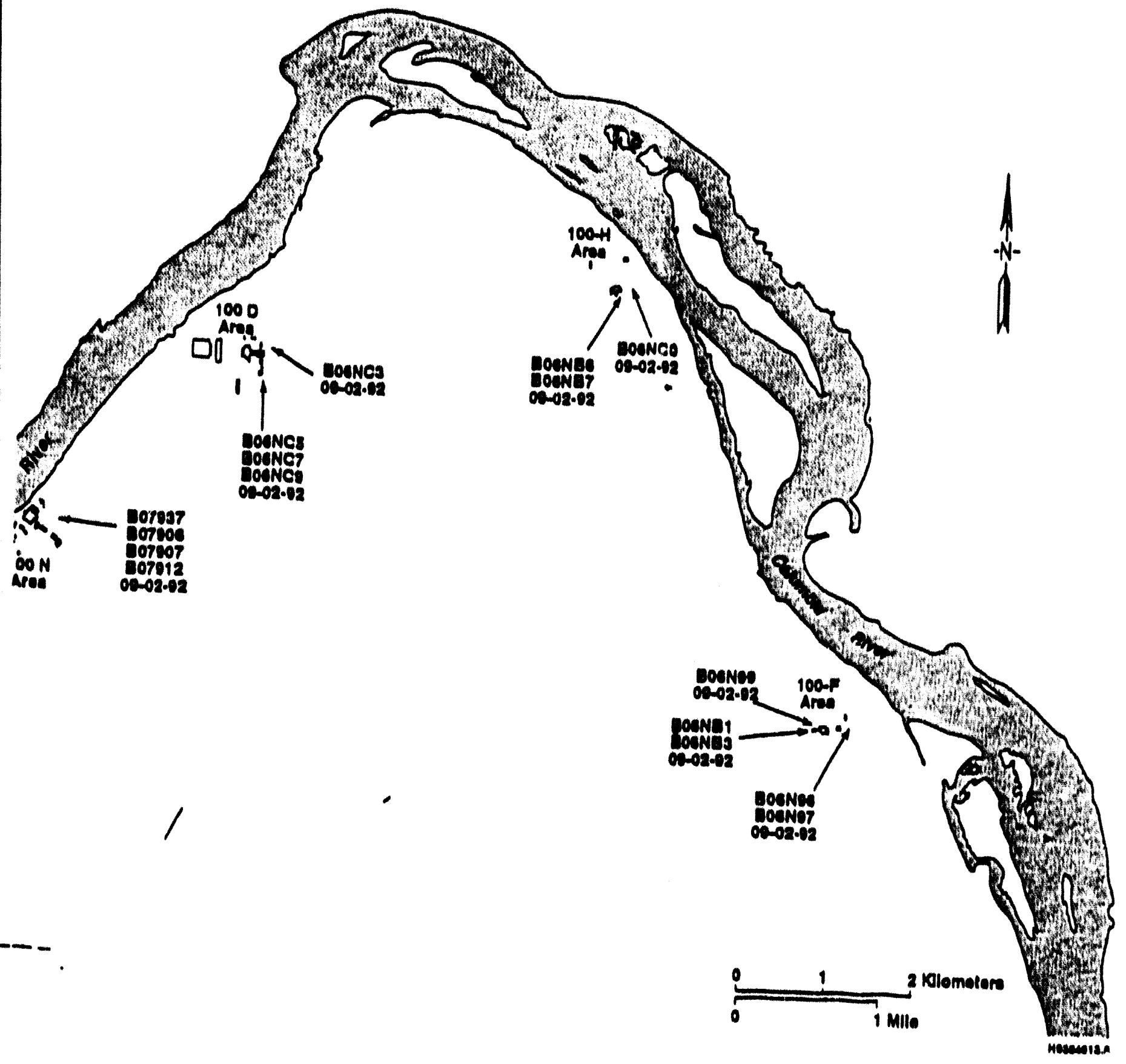


WHC-EP-0620

\section{APPENDIX B}

ANALYTICAL RESULTS FOR ASPARAGUS AND MILFOIL 
WHC-EP-0620

This page intentionally left blank. 
Table B-1. Asparagus Sample Results Collected from 100 Areas in 1991. (sheet 1 of 2)

\begin{tabular}{|c|c|c|c|c|c|c|c|c|}
\hline \multicolumn{9}{|c|}{ Sample Identification Numbers and Sample Locations } \\
\hline Conntitunt & Above $H$ & Above $H$ & Below D & Below D & Below D & Below D & Below D & Below D \\
\hline & Boov78 & Boov79 & BoOV80 & Bo0V81 & BOOV82 & Boov83 & BoOV84 & Boov85 \\
\hline Aluminum & $49.00 \mathrm{U}$ & $5.60 \mathrm{U}$ & $5.80 \mathrm{U}$ & $5.60 \mathrm{U}$ & $24.70 \mathrm{U}$ & $27.30 \mathrm{U}$ & $17.90 \mathrm{U}$ & $143.00 \mathrm{U}$ \\
\hline Ant imony & $3.60 \mathrm{U}$ & $3.50 \mathrm{U}$ & $3.70 \mathrm{~W}$ & $3.40 \mathrm{U}$ & $3.50 \mathrm{U}$ & $3.50 \mathrm{U}$ & $3.60 \mathrm{U}$ & $3.60 \mathrm{U}$ \\
\hline Arsenic & $0.84 \mathrm{UJ}$ & 0.83 UJ & $0.80 \mathrm{UJ}$ & $0.85 \mathrm{uJ}$ & $0.83 \mathrm{uJ}$ & 0.81 UJ & $4.00 \mathrm{UJ}$ & $4.20 \mathrm{UJ}$ \\
\hline Barium & $3.50 \mathrm{U}$ & $3.00 \mathrm{U}$ & $2.30 \mathrm{U}$ & $1.90 \mathrm{U}$ & $4.80 \mathrm{U}$ & $2.20 \mathrm{U}$ & $1.90 \mathrm{U}$ & $6.00 \mathrm{U}$ \\
\hline Beryllium & $0.21 \mathrm{U}$ & $0.21 \mathrm{U}$ & $0.21 \mathrm{U}$ & $0.20 \mathrm{U}$ & $0.20 \mathrm{U}$ & $0.21 \mathrm{U}$ & $0.21 \mathrm{U}$ & $0.21 \mathrm{U}$ \\
\hline Cadnium & $0.63 \mathrm{U}$ & $0.62 \mathrm{U}$ & $0.64 \mathrm{U}$ & $0.60 \mathrm{U}$ & $0.61 \mathrm{U}$ & $0.63 U$ & $0.63 U$ & $0.63 \mathrm{U}$ \\
\hline Calcium & 1360,00 & 1320.00 & $1050.00 \mathrm{~B}$ & 1350.00 & 1620.00 & $925.00 \mathrm{~B}$ & 1330.00 & 1570.00 \\
\hline Chromium & $1.50 \mathrm{U}$ & $1.50 \mathrm{U}$ & $1.50 \mathrm{U}$ & $1.40 \mathrm{U}$ & $1.40 \mathrm{U}$ & $1.50 \mathrm{U}$ & $1.50 \mathrm{U}$ & $1.50 \mathrm{U}$ \\
\hline Cobalt & $0.84 \mathrm{U}$ & $0.83 \mathrm{U}$ & $0.86 U$ & $0.79 \mathrm{U}$ & $0.82 \mathrm{U}$ & $0.84 \mathrm{U}$ & $0.85 \mathrm{U}$ & $0.84 \mathrm{U}$ \\
\hline Copper & 9.00 & 7.70 & 8.10 & 6.90 & 8.70 & 6.60 & $5.20 \mathrm{~B}$ & 5.50 \\
\hline Iron & $112.00 \mathrm{~J}$ & $37.20 \mathrm{~J}$ & $32.20 \mathrm{~J}$ & $27.30 \mathrm{~J}$ & $68.10 \mathrm{~J}$ & $85.00 \mathrm{~J}$ & $55.20 \mathrm{~J}$ & $294.00 \mathrm{~J}$ \\
\hline Leed & $0.38 \mathrm{~J}$ & $0.23 \mathrm{~J}$ & $0.28 \mathrm{~J}$ & $0.38 \mathrm{~J}$ & $0.29 \mathrm{~J}$ & $0.62 \mathrm{~J}$ & $0.35 \mathrm{~J}$ & $0.80 \mathrm{~J}$ \\
\hline Magnesium & 1180.00 & 1160.00 & $812.00 \mathrm{~B}$ & 1020.00 & 1140.00 & 1310.00 & 1210.00 & 1260.00 \\
\hline Manganese & 11.40 & 9.90 & 7.70 & 7.70 & 12.20 & 9.70 & 10.20 & 15.20 \\
\hline Mercury & $0.10 \mathrm{UJ}$ & $0.10 \mathrm{UJ}$ & $0.10 \mathrm{uJ}$ & $0.10 \mathrm{UJ}$ & $0.10 \mathrm{UJ}$ & $0.10 \mathrm{uJ}$ & $0.10 \mathrm{Us}$ & $0.09 \mathrm{UJ}$ \\
\hline Nickel & $1.50 \mathrm{~B}$ & $1.70 \mathrm{~B}$ & $1.30 \mathrm{U}$ & $1.20 \mathrm{~B}$ & $1.20 \mathrm{U}$ & $2.70 \mathrm{~B}$ & $1.60 \mathrm{~B}$ & $1.40 \mathrm{~B}$ \\
\hline Potassium & 25900.00 & 22200.00 & 17200.00 & 23800.00 & 21900.00 & 24900.00 & 25400.00 & 23200.00 \\
\hline Selenium & $0.84 \mathrm{U}$ & $0.83 \mathrm{UJ}$ & 0.80 uJ & $0.85 \mathrm{UJ}$ & $0.83 \mathrm{UJ}$ & $0.81 \mathrm{U}$ & $0.79 \mathrm{uJ}$ & $4.20 \mathrm{U}$ \\
\hline silver & $0.84 \mathrm{U}$ & $0.83 U$ & $0.86 U$ & $0.79 \mathrm{U}$ & $0.82 \mathrm{U}$ & $0.84 U$ & $0.85 \mathrm{U}$ & $0.84 U$ \\
\hline Sodium & $162.00 \mathrm{U}$ & $104.00 \mathrm{U}$ & $355.00 \mathrm{~J}$ & $91.50 \mathrm{U}$ & $95.10 \mathrm{U}$ & $161.00 \mathrm{U}$ & $122.00 \mathrm{U}$ & $194.00 \mathrm{U}$ \\
\hline Thallium & $3.10 \mathrm{R}$ & $3.10 R$ & $0.60 R$ & $3.20 \mathrm{R}$ & $3.10 \mathrm{R}$ & $0.61 R$ & $3.00 R$ & $3.10 R$. \\
\hline Vanadium & $0.63 \mathrm{U}$ & $0.62 \mathrm{U}$ & $0.64 \mathrm{U}$ & $0.60 \mathrm{U}$ & $0.76 \mathrm{~B}$ & $0.63 U$ & $0.63 \mathrm{U}$ & $0.65 \mathrm{~B}$ \\
\hline Zinc & $50.30 \mathrm{U}$ & $45.80 \mathrm{U}$ & $36.30 \mathrm{U}$ & $40.30 \mathrm{U}$ & $40.60 \mathrm{U}$ & $50.30 \mathrm{U}$ & $43.00 \mathrm{U}$ & $50.10 \mathrm{U}$ \\
\hline Cyenide & NR & NR & NR & NR & NR & NR & NR & NR \\
\hline Cs-137 & $<.1868$ & $<.168$ & $<.1816$ & $<.2613$ & $<.1415$ & $<.1950$ & $<.0972$ & $<.1323$ \\
\hline Sr $=90$ & $<.2254$ UJ & $<.2108$ UJ & $<.2172$ UJ & $<.2160 \mathrm{UJ}$ & $<.2137 \mathrm{UJ}$ & $<.2001 \mathrm{UJ}$ & $<.2290$ UJ & $<.1992$ UJ \\
\hline Tc- 99 & $<.25$ & $<.25 \mathrm{~J}$ & $<.37 \mathrm{~J}$ & $<.25 \mathrm{~J}$ & $<.32$ & $<.33 \mathrm{~J}$ & $<.37$ & $<.37 \mathrm{~J}$ \\
\hline
\end{tabular}

NOTE: All metals are reported in $\mathrm{mg} / \mathrm{kg}$. Radionuclides are reported in $\mathrm{pCi} / \mathrm{g}$.

\footnotetext{
B = Analyte found in associated blank as well as sample.

$J=$ Not detected; associated value is estimated.

NR = Nonreportable.

$R$ - Data are unusable.

$U=$ Not detected; value reported is sample quantitation limit.

UJ = Not detected; may not accurately reflect sample quantitation limit.
} 
Table B-1. Asparagus Sample Results Collected from 100 Areas in 1991. (sheet 2 of 2)

\begin{tabular}{|c|c|c|c|c|c|c|}
\hline \multicolumn{7}{|c|}{ Sample Identification Numbers and Sample Locations } \\
\hline Conetiturent & Below D & Below D & Control & Below $\mathrm{H}$ & Control & Control \\
\hline & BOOV86 & Boov87 & BoOV88 & Bo0v89 & Boov90 & Boov91 \\
\hline Aluminum & $33.60 \mathrm{U}$ & $18.60 \mathrm{U}$ & $35.70 \mathrm{U}$ & $17.10 \mathrm{U}$ & $20.60 \mathrm{U}$ & $11.00 \mathrm{U}$ \\
\hline Ant imony & $3.50 \mathrm{U}$ & $3.70 \mathrm{U}$ & $3.40 \mathrm{U}$ & $3.40 \mathrm{U}$ & $3.40 \mathrm{U}$ & $3.50 \mathrm{U}$ \\
\hline Arsenic & $4.00 \mathrm{UJ}$ & 0.82 us & 0.84 uJ & 0.85 UJ & $0.83 \mathrm{UJ}$ & $0.90 \mathrm{UJ}$ \\
\hline Berium & $3.90 \mathrm{U}$ & $2.10 \mathrm{U}$ & $5.60 \mathrm{U}$ & $2.90 \mathrm{U}$ & $4.80 \mathrm{U}$ & $1.70 \mathrm{U}$ \\
\hline Beryllium & $0.21 \mathrm{U}$ & $0.22 \mathrm{U}$ & $0.20 \mathrm{U}$ & $0.20 u$ & $0.25 U$ & $0.21 U$ \\
\hline Cachium & $0.62 U$ & $0.64 \mathrm{U}$ & $0.60 \mathrm{U}$ & $0.59 \mathrm{U}$ & $0.60 \mathrm{U}$ & $0.62 \mathrm{U}$ \\
\hline Calciun & 1580.00 & 1080.00 & 1550.00 & 1050.00 & 2040.00 & 1880.00 \\
\hline Chromium & $1.40 \mathrm{U}$ & $1.50 \mathrm{U}$ & $1.40 \mathrm{U}$ & $1.40 \mathrm{U}$ & $1.40 \mathrm{U}$ & $1.50 \mathrm{U}$ \\
\hline Cobalt & $0.83 \mathrm{U}$ & $0.86 \mathrm{U}$ & $0.79 \mathrm{U}$ & $0.79 \mathrm{U}$ & $0.80 \mathrm{U}$ & $0.83 \mathrm{U}$ \\
\hline Copper & $5.10 \mathrm{~B}$ & 6.30 & 5.50 & 6.00 & 5.90 & 7.20 \\
\hline Iron & $81.80 \mathrm{~J}$ & $50.00 \mathrm{~J}$ & $65.40 \mathrm{~J}$ & $64.80 \mathrm{~J}$ & $50.60 \mathrm{~J}$ & $40.40 \mathrm{~J}$ \\
\hline Lead & $0.41 \mathrm{~J}$ & $0.34 \mathrm{~J}$ & $0.40 \mathrm{~J}$ & $0.40 \mathrm{~B}$ & $0.42 \mathrm{~J}$ & $0.64 \mathrm{~J}$ \\
\hline Magnesium & 1310.00 & $1030.00 \mathrm{~B}$ & $984.00 \mathrm{~B}$ & 1010.00 & $976.00 \mathrm{~B}$ & 1120.00 \\
\hline Manganese & 13.90 & 11.00 & 8.80 & 8.80 & 9.50 & 17.80 \\
\hline Mercury & $0.10 \mathrm{uJ}$ & 0.09 UJ & $0.09 \mathrm{uJ}$ & 0.09 UJ & $0.10 \mathrm{UJ}$ & $0.10 \mathrm{UJ}$ \\
\hline Nickel & $2.10 \mathrm{~B}$ & $1.30 \mathrm{U}$ & $1.30 \mathrm{~B}$ & $1.20 \mathrm{~B}$ & $1.20 \mathrm{U}$ & $2.20 \mathrm{~B}$ \\
\hline Potassium & 23900.00 & 22700.00 & 20300.00 & 19500.00 & 20600.00 & 25900.00 \\
\hline Selenium & 0.80 us & $0.82 \mathrm{UJ}$ & $0.84 \mathrm{U}$ & $0.85 \mathrm{uJ}$ & $0.83 \mathrm{UJ}$ & $4.50 \mathrm{U}$ \\
\hline silver & $0.83 U$ & $0.86 U$ & $0.79 \mathrm{U}$ & $0.79 \mathrm{U}$ & $0.80 \mathrm{U}$ & $0.83 \mathrm{U}$ \\
\hline Sodium & $145.00 \mathrm{U}$ & $102.00 \mathrm{U}$ & $116.00 \mathrm{U}$ & $168.00 \mathrm{U}$ & $141.00 \mathrm{U}$ & $106.00 \mathrm{U}$ \\
\hline Thallium & $3.00 \mathrm{R}$ & $3.10 \mathrm{R}$ & $0.63 R$ & $0.64 R$ & $0.62 R$ & $0.67 R$ \\
\hline Vanadium & 0.77 B & $0.80 \mathrm{~B}$ & $0.60 \mathrm{U}$ & $0.59 \mathrm{U}$ & $0.60 \mathrm{U}$ & $0.62 \mathrm{U}$ \\
\hline Zine & $49.00 \mathrm{U}$ & $39.00 \mathrm{U}$ & $41.70 \mathrm{U}$ & $43.90 \mathrm{U}$ & $32.40 \mathrm{U}$ & $39.50 \mathrm{U}$ \\
\hline Cyanide & NR & NR & NR & NR & NR & NR \\
\hline Cs- 137 & $<, 1589$ & $<.2297$ & $<.1082$ & $<.1914$ & $<.2666$ & $<.1690$ \\
\hline $5 r-90$ & $<.2241 \mathrm{UJ}$ & $<.2270$ UJ & $<.2162 \mathrm{UJ}$ & $<.2812 \mathrm{UJ}$ & $<.1478$ UJ & $<.1538$ UJ \\
\hline Tc- 99 & $<.22 \mathrm{~J}$ & $<.12$ & $<.28$ & $<.24$ & $<.29$ & $<.22$ \\
\hline
\end{tabular}

NOTE: All metals are reported in $\mathrm{mg} / \mathrm{kg}$. Radionuclides are reported in $\mathrm{pCi} / \mathrm{g}$.

$B=$ Analyte found in associated blank as well as sample.

$J$ = Not detected; associated value is estimated.

NR = Nonreportable.

$R=$ Date are unusable.

$U=$ Not detected; volue reported is sample quantitation limit.

UJ = Not detected; may not accurataly reflect sample quantitation limit. 
Table B-2. Asparagus Sample Results Collected from 100 Areas in 1992.

\begin{tabular}{|c|c|c|c|c|c|c|c|c|}
\hline \multicolumn{9}{|c|}{ Sample Identification Numbers and Sample Locations } \\
\hline \multirow{2}{*}{ Const i tuent } & Below F & Above $F$ & Above $\mathrm{N}$ & Below K & Above $\mathrm{K}$ & Above $F$ & Control & Control \\
\hline & B06N41 & B06N42 & $806 N 43$ & B06N44 & $806 N 45$ & B06N46 & B06N47 & $806 N 48$ \\
\hline Aluninum & $25.10 \mathrm{U}$ & $19.50 \mathrm{U}$ & $21.90 \mathrm{U}$ & $21.10 \mathrm{U}$ & $18.10 \mathrm{U}$ & $20.50 \mathrm{U}$ & $21.10 \mathrm{U}$ & $15.90 \mathrm{U}$ \\
\hline Antimony & $13.20 \mathrm{U}$ & $10.20 \mathrm{U}$ & $11.50 \mathrm{U}$ & $11.00 \mathrm{U}$ & $10.50 \mathrm{U}$ & $10.80 U$ & $12.70 \mathrm{U}$ & $8.30 \mathrm{U}$ \\
\hline Arsenic & $7.50 \mathrm{~J}$ & $2.90 \mathrm{U}$ & $4.00 \mathrm{U}$ & $3.50 \mathrm{U}$ & $2.70 \mathrm{us}$ & $2.10 \mathrm{UJ}$ & $2.10 \mathrm{U}$ & $1.70 \mathrm{U}$ \\
\hline Barium & $8.00 \mathrm{~B}$ & $4.70 \mathrm{U}$ & $5.20 \mathrm{U}$ & $5.00 \mathrm{U}$ & $5.80 \mathrm{~B}$ & $4.90 \mathrm{U}$ & $5.00 \mathrm{U}$ & $6.10 \mathrm{~B}$ \\
\hline Beryllium & $1.20 \mathrm{U}$ & $0.93 \mathrm{U}$ & $1.00 \mathrm{U}$ & $1.00 \mathrm{U}$ & $0.86 \mathrm{U}$ & $0.98 \mathrm{U}$ & $1.00 \mathrm{U}$ & $0.76 \mathrm{U}$ \\
\hline Cactnium & $2.60 \mathrm{U}$ & $1.50 \mathrm{U}$ & $1.00 \mathrm{U}$ & $1.00 \mathrm{U}$ & $1.40 \mathrm{U}$ & $3.40 \mathrm{U}$ & $1.00 \mathrm{U}$ & $0.76 \mathrm{U}$ \\
\hline Calcium & $1500.00 \mathrm{~B}$ & $1810.00 \mathrm{~B}$ & $1430.00 \mathrm{~B}$ & $2010.00 \mathrm{~B}$ & $2000.00 \mathrm{~B}$ & $1410.00 \mathrm{~B}$ & $1130.00 \mathrm{~B}$ & $1610.00 \mathrm{~B}$ \\
\hline Chromium & $6.00 \mathrm{U}$ & $4.70 \mathrm{U}$ & $5.20 \mathrm{U}$ & $5.00 \mathrm{U}$ & $4.30 \mathrm{~V}$ & $4.90 \mathrm{U}$ & $5.00 \mathrm{U}$ & $3.80 \mathrm{U}$ \\
\hline Cobalt & $2.40 \mathrm{U}$ & $1.90 \mathrm{U}$ & $2.10 \mathrm{U}$ & $2.00 \mathrm{U}$ & $1.70 \mathrm{U}$ & $2.00 \mathrm{U}$ & $2.00 \mathrm{U}$ & $1.50 \mathrm{U}$ \\
\hline Copper & $12.20 \mathrm{~B}$ & $8.80 \mathrm{~B}$ & $9.00 \mathrm{~B}$ & $14.20 \mathrm{~B}$ & $9.50 \mathrm{~B}$ & $10.00 \mathrm{~B}$ & $10.30 \mathrm{~B}$ & $5.40 \mathrm{U}$ \\
\hline Iron & $85.60 \mathrm{~B}$ & $39.60 \mathrm{~B}$ & $60.00 \mathrm{~B}$ & $42.20 \mathrm{~B}$ & $36.70 \mathrm{~B}$ & $37.10 \mathrm{~g}$ & $28.90 \mathrm{~B}$ & $29.00 \mathrm{~B}$ \\
\hline Lead & $1.90 \mathrm{~B}$ & $1.00 \mathrm{~J}$ & $1.40 \mathrm{~B}$ & $1.40 \mathrm{~J}$ & $1.90 \mathrm{~B}$ & $1.80 \mathrm{~J}$ & $1.20 \mathrm{~B}$ & $1.20 \mathrm{~J}$ \\
\hline Magnesium & $810.00 \mathrm{~B}$ & $863.00 \mathrm{~B}$ & $838.00 \mathrm{~B}$ & $920.00 \mathrm{~B}$ & $892.00 \mathrm{~B}$ & $767.00 \mathrm{~B}$ & $788.00 \mathrm{~B}$ & $682.00 \mathrm{~B}$ \\
\hline Manganese & $12.90 \mathrm{~B}$ & $8.20 \mathrm{~B}$ & $6.60 \mathrm{~B}$ & $9.80 \mathrm{~B}$ & $9.10 \mathrm{~B}$ & $11.20 \mathrm{~B}$ & $10.30 \mathrm{~B}$ & $5.50 \mathrm{~B}$ \\
\hline Mercury & $0.53 \mathrm{UJ}$ & $0.42 \mathrm{UJ}$ & $0.58 \mathrm{UJ}$ & $0.48 \mathrm{UJ}$ & $0.44 \mathrm{UJ}$ & $0.43 \mathrm{UJ}$ & $1.50 \mathrm{~J}$ & $0.37 \mathrm{~J}$ \\
\hline Nickel & $6.00 \mathrm{U}$ & $4.70 \mathrm{U}$ & $5.20 \mathrm{U}$ & $5.50 \mathrm{~B}$ & $4.30 \mathrm{U}$ & $4.90 \mathrm{U}$ & $5.00 \mathrm{U}$ & $3.80 \mathrm{U}$ \\
\hline Potassium & 26600.00 & 22100.00 & 21100.00 & 20400.00 & 20400.00 & 19100.00 & 20800.00 & 19500.00 \\
\hline Selenium & $4.80 \mathrm{~J}$ & $3.40 \mathrm{UJ}$ & $4.10 \mathrm{UJ}$ & $4.30 \mathrm{U}$ & $3.70 \mathrm{UJ}$ & $4.10 \mathrm{~J}$ & $3.90 \mathrm{U}$ & $3.00 \mathrm{U}$ \\
\hline silver & $3.60 \mathrm{U}$ & $2.80 \mathrm{U}$ & $3.10 \mathrm{U}$ & $3.00 \mathrm{U}$ & $2.60 \mathrm{U}$ & $2.90 \mathrm{U}$ & $3.00 \mathrm{U}$ & $2.30 \mathrm{U}$ \\
\hline Sodium & $186.00 \mathrm{~B}$ & $143.00 \mathrm{~B}$ & $124.00 \mathrm{~B}$ & $183.00 \mathrm{~B}$ & $147.00 \mathrm{~B}$ & $140.00 \mathrm{~B}$ & $189.00 \mathrm{~B}$ & 134.008 \\
\hline Thallium & $1.10 \mathrm{UJ}$ & $0.85 \mathrm{UJ}$ & $1.00 \mathrm{UJ}$ & $5.40 \mathrm{UJ}$ & $0.93 \mathrm{uJ}$ & $0.91 \mathrm{UJ}$ & 0.99 UJ & $0.74 \mathrm{UJ}$ \\
\hline Vanadium & $2.40 \mathrm{U}$ & $1.90 \mathrm{U}$ & $2.10 \mathrm{U}$ & $2.00 \mathrm{U}$ & $1.70 \mathrm{U}$ & $2.00 \mathrm{U}$ & $2.00 \mathrm{U}$ & $1.50 \mathrm{U}$ \\
\hline 2 inc & 97.20 & 95.00 & 73.40 & 69.30 & 59.20 & 97.40 & 62.40 & 61.10 \\
\hline Cyanide & NR & NR & NR & NR & NR & NR & NR & $N R$ \\
\hline Cs-137 & $u$ & u & $U$ & $u$ & $u$ & $u$ & $u$ & $u$ \\
\hline $5 r-90$ & 0.048 & 0.330 & -0.280 & -0.130 & 0.150 & -0.024 & 0.001 & -0.043 \\
\hline
\end{tabular}

NOTE: All metals are reported in mg/kg. Radionuclides are reported in pci/g.

$B$ = Analyte found in associated blank as well as sample.

$J=$ Not detected; associated value is estimated.

NR = Monreportable.

$R=$ Data are unusable.

$U$ = Not detected; value reported is sample quantitation limit.

UJ = Not detected; may not accurately reflect sample quantitation I imit. 
Table B-3. Milfoil Results Collected at N Springs in 1992.

\begin{tabular}{|c|c|c|c|c|}
\hline \multirow{2}{*}{ Const i tuent } & \multicolumn{4}{|c|}{ Sample Identification Numbers and Sample Locations } \\
\hline & B07921 & B07922 & B07923 & $\begin{array}{l}\text { Control } \\
\text { B07924 }\end{array}$ \\
\hline Aluminum & 6860.00 & 2760.00 & 3740.00 & 987.00 \\
\hline Antimony & $9.20 \mathrm{U}$ & $5.20 \mathrm{U}$ & $5.40 \mathrm{U}$ & $19.60 \mathrm{U}$ \\
\hline Arsenic & 6.30 & $2.90 \mathrm{~B}$ & 4.00 & $3.80 \mathrm{UJ}$ \\
\hline Bariun & 147.00 & 104.00 & 122.00 & $114.00 \mathrm{~B}$ \\
\hline Beryllium & $0.64 \mathrm{U}$ & $0.12 U$ & $0.38 \mathrm{U}$ & $0.63 \mathrm{U}$ \\
\hline Codnium & 4.60 & 4.30 & 4.60 & $5.50 \mathrm{~B}$ \\
\hline Colcium & $21100.00 \mathrm{~J}$ & $21700.00 \mathrm{~J}$ & $20500.00 \mathrm{~J}$ & $26200.00 \mathrm{~J}$ \\
\hline Chromium & 16.20 & 5.40 & 8.20 & $7.00 \mathrm{~B}$ \\
\hline Cobalt & $5.20 \mathrm{~B}$ & $2.00 \mathrm{~B}$ & $3.10 \mathrm{~B}$ & $3.10 \mathrm{U}$ \\
\hline Copper & 61.10 & 24.90 & 35.70 & $26.00 \mathrm{~B}$ \\
\hline Iron & 12200.00 & 4190.00 & 6400.00 & 1780.00 \\
\hline Lead & 28.30 & 11.60 & 17.70 & 9.80 \\
\hline Magnesium & $6050.00 \mathrm{~J}$ & $3390.00 \mathrm{~J}$ & $3620.00 \mathrm{~J}$ & $2650.00 \mathrm{~J}$ \\
\hline Manganese & 1190.00 & 402.00 & 771.00 & 370.00 \\
\hline Mercury & 0.45 & 0.16 & 0.27 & $0.47 \mathrm{~B}$ \\
\hline Nickel & $16.40 \mathrm{~B}$ & $6.20 \mathrm{~B}$ & $9.30 \mathrm{~B}$ & $9.00 \mathrm{~B}$ \\
\hline Potassium & 7840.00 & $1110.00 \mathrm{~B}$ & 4500.00 & $2570.00 \mathrm{~B}$ \\
\hline Selenium & $2.10 \mathrm{U}$ & $1.40 \mathrm{U}$ & $1.20 \mathrm{U}$ & $4.60 \mathrm{U}$ \\
\hline silver & $1.40 \mathrm{U}$ & $0.77 \mathrm{u}$ & $0.81 \mathrm{U}$ & $2.90 \mathrm{U}$ \\
\hline Sodium & $2900.00 \mathrm{~J}$ & $464.00 \mathrm{~J}$ & $1910.00 \mathrm{~J}$ & $1190.00 \mathrm{~J}$ \\
\hline Thallium & $2.10 \mathrm{U}$ & $1.20 \mathrm{UJ}$ & $1.20 \mathrm{UJ}$ & $4.50 \mathrm{UJ}$ \\
\hline Vanadium & $20.20 \mathrm{~B}$ & $9.20 \mathrm{~B}$ & $11.80 \mathrm{~B}$ & $5.40 \mathrm{~B}$ \\
\hline Zine & $319.00 \mathrm{~J}$ & $189.00 \mathrm{~J}$ & $230.00 \mathrm{~J}$ & $206.00 \mathrm{~J}$ \\
\hline Cyanide & NR & NR & NR & NR \\
\hline C8-137 & $u$ & u & 0.19 & $u$ \\
\hline $5 r-90$ & $0.18 R$ & $.0 .16 R$ & 0.61 & -0.04 \\
\hline
\end{tabular}

NOTE: All metals are reported in $\mathrm{mg} / \mathrm{kg}$. Radionucllides are reported in $\mathrm{PCi} / \mathrm{gm}$.

\footnotetext{
B = Analyte found in associated blank as well as sample.

$J$ - Not detected; associated value is estimated.

NR = Nonreportable.

$R$ = Data are unusable.

$U$ - Not detected; value reported is sample quantitation limit.

UJ = Not detected; may not accurately roflect sample quantitation

Iimit.
} 
WHC-EP-0620

APPENDIX C ANALYTICAL RESULTS FOR REED CANARYGRASS 
WHC-EP-0620

This page intentionally left blank. 
Table C-1. Reed Canarygrass Sample Results from 100-F Reactor Area Collected Downriver in 1991.

\begin{tabular}{|c|c|c|c|c|c|c|}
\hline \multirow{2}{*}{ Const i tuent } & \multicolumn{6}{|c|}{ Sample Identifleation Numbers } \\
\hline & Boovz1 & Boovz2 & BoOVz3 & Boovz4 & BoOVzs & $800 \mathrm{Vz} 6$ \\
\hline Aluminum & $704.00 \mathrm{~J}$ & $456.00 \mathrm{~J}$ & $548.00 \mathrm{~J}$ & $1650.00 \mathrm{~J}$ & $691.00 \mathrm{~J}$ & $301.00 \mathrm{~J}$ \\
\hline Antimony & $6.80 \mathrm{UJ}$ & $6.60 \mathrm{UJ}$ & $6.50 \mathrm{UJ}$ & 6.40 Us & $6.70 \mathrm{UJ}$ & 6.30 UJ \\
\hline Arsenic & $0.89 \mathrm{UJ}$ & 0.75 Us & $0.77 \mathrm{UJ}$ & $1.40 \mathrm{UJ}$ & $0.52 \mathrm{UJ}$ & $0.41 \mathrm{UJ}$ \\
\hline Bariun & $25.90 \mathrm{~J}$ & $19.60 \mathrm{~J}$ & $18.00 \mathrm{~J}$ & $44.50 \mathrm{~J}$ & $21.50 \mathrm{~J}$ & $21.30 \mathrm{~J}$ \\
\hline Boryllium & 0.22 uJ & 0.21 UJ & 0.21 UJ & $0.21 \mathrm{UJ}$ & 0.22 UJ & $0.20 \mathrm{UJ}$ \\
\hline Cectaium & $0.53 \mathrm{UJ}$ & $0.58 \mathrm{UJ}$ & $0.33 \mathrm{UJ}$ & 0.88 UJ & 0.37 UJ & 0.37 UJ \\
\hline Colciun & $3880.00 \mathrm{~J}$ & $3020.00 \mathrm{~J}$ & $3160.00 \mathrm{~J}$ & $3840.00 \mathrm{~J}$ & $3520.00 \mathrm{~J}$ & $3360.00 \mathrm{~J}$ \\
\hline Chromiun & $3.00 \mathrm{UN}$ & 2.10 UN & $2.70 \mathrm{UJ}$ & $5.10 \mathrm{~J}$ & $2.90 \mathrm{UJ}$ & $4.00 \mathrm{UJ}$ \\
\hline Cobalt & $0.65 \mathrm{~J}$ & $0.63 \mathrm{UJ}$ & $0.63 \mathrm{UJ}$ & $2.00 \mathrm{~J}$ & $0.65 \mathrm{~J}$ & $0.61 \mathrm{UJ}$ \\
\hline Copper & $10.50 \mathrm{~J}$ & $9.10 \mathrm{~J}$ & $10.70 \mathrm{~J}$ & $18.60 \mathrm{~J}$ & $7.50 \mathrm{UJ}$ & $9.20 \mathrm{UJ}$ \\
\hline Iron & $1230.00 \mathrm{~J}$ & $774.00 \mathrm{~J}$ & $994.00 \mathrm{~J}$ & $2910.00 \mathrm{~J}$ & $1220.00 \mathrm{~J}$ & $609.00 \mathrm{~J}$ \\
\hline Lead & $0.27 \mathrm{UJ}$ & $0.59 \mathrm{UJ}$ & 0.40 UJ & 0.37 UJ & $0.22 \mathrm{UJ}$ & $0.38 \mathrm{UJ}$ \\
\hline Magnes Iun & $1900.00 \mathrm{~J}$ & $1410.00 \mathrm{~J}$ & $1790.00 \mathrm{~J}$ & $3240.00 \mathrm{~J}$ & $1620.00 \mathrm{~J}$ & $1690.00 \mathrm{~J}$ \\
\hline Mengenese & $125.00 \mathrm{~J}$ & $85.50 \mathrm{~J}$ & $\pi .50 \mathrm{~J}$ & $199.00 \mathrm{~J}$ & $113.00 \mathrm{~J}$ & $65.10 \mathrm{~J}$ \\
\hline Mercury & $0.09 \mathrm{Ud}$ & $0.10 \mathrm{UJ}$ & $0.10 \mathrm{UJ}$ & 0.09 us & 0.09 UJ & $0.10 \mathrm{us}$ \\
\hline Nickel & $2.10 \mathrm{~J}$ & $1.00 \mathrm{~J}$ & $1.20 \mathrm{~J}$ & $5.20 \mathrm{~J}$ & $2.10 \mathrm{~J}$ & $0.92 \mathrm{~J}$ \\
\hline Potassiun & $18400.00 \mathrm{~J}$ & $16500.00 \mathrm{~J}$ & $23300.00 \mathrm{~J}$ & $24900.00 \mathrm{~J}$ & $12600.00 \mathrm{~J}$ & $20600.00 \mathrm{~J}$ \\
\hline seleniun & $0.85 \mathrm{UJ}$ & $4.10 \mathrm{UJ}$ & $4.20 \mathrm{UJ}$ & $4.30 \mathrm{UJ}$ & 0.87 UJ & $0.83 \mathrm{UJ}$ \\
\hline silver & $1.10 \mathrm{UJ}$ & $1.10 \mathrm{UJ}$ & $1.10 \mathrm{UJ}$ & $1.00 \mathrm{UJ}$ & $1.10 \mathrm{UJ}$ & $1.00 \mathrm{Ud}$ \\
\hline sodium & $172.00 \mathrm{~J}$ & $147.00 \mathrm{~J}$ & $155.00 \mathrm{~J}$ & $369.00 \mathrm{~J}$ & $197.00 \mathrm{~J}$ & $62.50 \mathrm{U}$ \\
\hline Thallium & $0.43 \mathrm{Ud}$ & $0.41 \mathrm{UJ}$ & 0.42 UJ & $0.43 \mathrm{UJ}$ & $0.43 \mathrm{UJ}$ & $0.41 \mathrm{UJ}$ \\
\hline Vanodlum & $2.50 \mathrm{~J}$ & $1.50 \mathrm{~J}$ & $1.80 \mathrm{~J}$ & $5.50 \mathrm{~J}$ & $2.40 \mathrm{~J}$ & $0.98 \mathrm{~J}$ \\
\hline zinc & $64.30 \mathrm{~J}$ & $96.00 \mathrm{~J}$ & $155.00 \mathrm{~J}$ & $140.00 \mathrm{~J}$ & $70.40 \mathrm{~J}$ & $89.30 \mathrm{~J}$ \\
\hline Cyonide & NR & NR & NR & NR & NR & NR \\
\hline $68 \cdot 137$ & $<.5132$ UJ & $0.76 \mathrm{~J}$ & $<1.270$ UJ & $<1.126 \mathrm{UJ}$ & $0.85 \mathrm{~J}$ & $\$ 1.078$ US \\
\hline $8 r .90$ & $-0.40 \mathrm{UJ}$ & $0.05 \mathrm{UJ}$ & -0.04 UJ & $-0.03 \mathrm{UJ}$ & $-0.04 U_{N}$ & $-0.06 \mathrm{UJ}$ \\
\hline Tc- 99 & 0.20 UJ & $0.40 \mathrm{UJ}$ & $0.30 \mathrm{UJ}$ & $0.50 \mathrm{~J}$ & $0.30 \mathrm{R}$ & $0.30 \mathrm{UJ}$ \\
\hline
\end{tabular}

NOTE: All motals ore reported in mo/kg. Radionuclldes ore reported in pci/o.

B - Analye found in assoclated blank as well as sample.

$J$ - Not detected; associated value is estimated.

Wh - Nonreportable.

$R$ - Dote are unusable.

$U$ - Not datected; value reported is sample quantitation IImit.

UJ - Not detected; may not accurately reflect semple quantitation Iimit. 
Table C-2. Reed Canarygrass Sample Results from 100-H Reactor Area Collected Upriver in 1991.

\begin{tabular}{|c|c|c|c|c|c|c|}
\hline \multirow{2}{*}{ Const I tuent } & \multicolumn{6}{|c|}{ Semplo Identifleation Numbers } \\
\hline & Boovxs & Boovx6 & BoovX7 & $800 \mathrm{~V} \times 8$ & BOOVX9 & BoovYo \\
\hline Aluminum & $11.60 \mathrm{UJ}$ & $556.00 \mathrm{~J}$ & $12.90 \mathrm{UJ}$ & $8.40 \mathrm{UJ}$ & 25.10 UJ & $18.30 \mathrm{UJ}$ \\
\hline Ant imony & $1.60 \mathrm{UJ}$ & $1.60 \mathrm{UJ}$ & $1.70 \mathrm{UJ}$ & $1.60 \mathrm{UJ}$ & $1.60 \mathrm{UJ}$ & $1.70 \mathrm{UJ}$ \\
\hline Arsenic & $0.88 \mathrm{~d}$ & $0.90 \mathrm{~J}$ & $0.58 \mathrm{~J}$ & $0.40 \mathrm{UJ}$ & 0.43 US & $0.43 \mathrm{ud}$ \\
\hline Bariun & $35.70 \mathrm{~J}$ & $23.90 \mathrm{~J}$ & $14.60 \mathrm{~J}$ & $21.00 \mathrm{~J}$ & $34.60 \mathrm{~J}$ & $20.60 \mathrm{~J}$ \\
\hline Beryllium & 0.21 Ud & $0.20 \mathrm{UJ}$ & $0.21 \mathrm{UJ}$ & $0.20 \mathrm{UJ}$ & 0.21 us & $0.21 U_{N}$ \\
\hline Cectuium & 0.21 UJ & 0.35 US & $0.21 \mathrm{UJ}$ & $0.20 \mathrm{UJ}$ & $0.21 \mathrm{uJ}$ & 0.21 UN \\
\hline Calclum & $3330.00 \mathrm{~J}$ & $4960.00 \mathrm{~J}$ & $3010.00 \mathrm{~J}$ & $3620.00 \mathrm{~J}$ & $5790.00 \mathrm{~J}$ & $3510.00 \mathrm{~J}$ \\
\hline Chroniun & $0.82 \mathrm{UJ}$ & $2.30 \mathrm{~J}$ & 0.86 UJ & 0.81 UJ & $1.30 \mathrm{~J}$ & $1.60 \mathrm{~J}$ \\
\hline Cobolt & 0.62 us & 0.94 UJ & $0.64 \mathrm{UJ}$ & 0.61 UJ & 0.62 Ud & 0.64 UJ \\
\hline Copper & $5.80 \mathrm{UJ}$ & $12.70 \mathrm{~J}$ & $3.80 \mathrm{UJ}$ & $5.10 \mathrm{UJ}$ & $5.20 \mathrm{UJ}$ & $6.10 \mathrm{UN}$ \\
\hline Iron & $44.20 \mathrm{~J}$ & $986.00 \mathrm{~J}$ & $30.20 \mathrm{~J}$ & $37.60 \mathrm{~J}$ & $69.90 \mathrm{~J}$ & $62.00 \mathrm{~J}$ \\
\hline Lead & $0.43 \mathrm{UJ}$ & $2.20 \mathrm{~J}$ & $0.43 \mathrm{UJ}$ & 0.40 Us & 0.43 US & $2.10 \mathrm{UJ}$ \\
\hline Magnes Iun & $1360.00 \mathrm{~J}$ & $2720.00 \mathrm{~J}$ & $1630.00 \mathrm{~J}$ & $1660.00 \mathrm{~J}$ & $1770.00 \mathrm{~J}$ & $1830.00 \mathrm{~J}$ \\
\hline Mangeneces & $13.20 \mathrm{~J}$ & $69.20 \mathrm{~J}$ & $10.40 \mathrm{~J}$ & $27.50 \AA$ & $14.20 \mathrm{~J}$ & $13.20 \mathrm{~J}$ \\
\hline Mercury & 0.09 Ud & 0.10 UJ & $0.10 \mathrm{UJ}$ & $0.10 \mathrm{uJ}$ & $0.11 \mathrm{WJ}$ & $0.10 \mathrm{UJ}$ \\
\hline Nickol & 0.82 Ud & $2.30 \mathrm{UJ}$ & $1.60 \mathrm{UJ}$ & $1.20 \mathrm{UJ}$ & 0.82 UJ & $0.94 \mathrm{UJ}$ \\
\hline Potansium & $18300.00 \mathrm{~J}$ & $20000.00 \mathrm{~J}$ & $12800.00 \mathrm{UJ}$ & $18600.00 \mathrm{~J}$ & $16900.00 \mathrm{~J}$ & $18800.00 \mathrm{~J}$ \\
\hline selonium & 0.86 Us & $0.81 \mathrm{UJ}$ & $0.86 \mathrm{UJ}$ & $0.81 \mathrm{UJ}$ & $0.86 \mathrm{UJ}$ & 0.86 UJ \\
\hline silver & $0.41 \mathrm{UJ}$ & $0.40 \mathrm{UJ}$ & 0.43 UJ & $0.40 \mathrm{US}$ & $0.41 \mathrm{WJ}$ & $0.43 \mathrm{UJ}$ \\
\hline sodium & $64.90 \mathrm{~J}$ & $101.00 \mathrm{~J}$ & $37.60 \mathrm{UJ}$ & $79.40 \mathrm{~J}$ & $47.00 \mathrm{~J}$ & $22.40 \mathrm{UJ}$ \\
\hline Thalllum & $3.20 \mathrm{R}$ & $3.00 \mathrm{R}$ & $3.20 \mathrm{R}$ & $3.00 \mathrm{R}$ & $3.20 R$ & $3.20 \mathrm{R}$ \\
\hline Venadiun & $0.61 \mathrm{UJ}$ & $1.50 \mathrm{~J}$ & 0.43 UJ & $0.40 \mathrm{UJ}$ & $0.41 \mathrm{UJ}$ & $0.43 \mathrm{UJ}$ \\
\hline $2 \ln e$ & $96.80 \mathrm{~J}$ & $186.00 \mathrm{~J}$ & $89.80 \mathrm{~J}$ & $97.30 \mathrm{~J}$ & $84.40 \mathrm{~J}$ & $89.30 \mathrm{~J}$ \\
\hline Cyanide & Nh & NR & NR & NR & NR & MR \\
\hline$C 8 \cdot 137$ & $<1.063 \mathrm{ud}$ & $<1.630 \mathrm{UJ}$ & $<1.020 \mathrm{us}$ & $<.865$ UJ & $C .9501$ US & $\$ 1.456 \mathrm{UJ}$ \\
\hline $8 r \cdot 90$ & $-0.05 \mathrm{us}$ & $=0.3 \mathrm{UJ}$ & $-0.70 \mathrm{UJ}$ & $0.05 \mathrm{UJ}$ & 0.09 UJ & $0.03 \mathrm{UJ}$ \\
\hline 16.99 & $0.20 R$ & $0.20 \mathrm{UJ}$ & $0.30 \mathrm{UJ}$ & $0.20 R$ & $0.50 \mathrm{R}$ & $0.20 R$ \\
\hline
\end{tabular}

NOTE: All metals are reported in $\mathrm{mg} / \mathrm{kg}$. Radionucllides are reported in $\mathrm{pCl} / \mathrm{g}$.

8 - Analyte found in associated blank os woll as semple.

$J$ - Not detected; asepciated value is estimated.

NR - Nonreportable.

- Dote are ununable.

$U$ - Not detected volue reported is sample quantitation IImit.

UJ a Not dotected; may not accurately reflect sample quantitation limit. 
Table C-3. Reed Canarygrass Sample Results from 100 H Reactor Area Collected Downriver in 1991.

\begin{tabular}{|c|c|c|c|c|c|c|}
\hline \multirow{2}{*}{ Const I tuent } & \multicolumn{6}{|c|}{ semple Idantification Numbers } \\
\hline & soovr3 & BOOVY4 & BoOVYs & BOOVY6 & BoOVY7 & BoOVY8 \\
\hline Aluminum & $10.20 \mathrm{UJ}$ & 7.90 UJ & $883.00 \mathrm{~J}$ & $843.00 \mathrm{~J}$ & $3020.00 \mathrm{~J}$ & $315.00 \mathrm{~J}$ \\
\hline Ant imony & $1.80 \mathrm{Ud}$ & $1.70 \mathrm{UJ}$ & $1.70 \mathrm{Ud}$ & $1.70 \mathrm{us}$ & $1.70 \mathrm{UJ}$ & $1.70 \mathrm{UJ}$ \\
\hline Arsenic & $0.94 \mathrm{~J}$ & $0.88 \mathrm{~J}$ & $1.30 \mathrm{~J}$ & $1.30 \mathrm{~J}$ & $3.50 \mathrm{~J}$ & $0.84 \mathrm{~J}$ \\
\hline Darlua & $10.00 \mathrm{~J}$ & $13.20 \mathrm{~N}$ & $33.80 \mathrm{~J}$ & $23.10 \mathrm{~J}$ & $52.10 \mathrm{~J}$ & $26.20 \mathrm{~J}$ \\
\hline Berylllum & $0.22 \mathrm{UJ}$ & 0.22 UJ & 0.21 UJ & 0.22 UN & $0.21 \mathrm{UJ}$ & $0.21 \mathrm{UJ}$ \\
\hline Cectulum & $0.22 \mathrm{UJ}$ & $0.22 \mathrm{UJ}$ & $0.89 \mathrm{UJ}$ & $0.54 \mathrm{UJ}$ & $0.94 \mathrm{UJ}$ & $0.24 \mathrm{UJ}$ \\
\hline Calclun & $2560.00 \mathrm{~J}$ & $2110.00 \mathrm{~J}$ & $4330.00 \mathrm{~J}$ & $3690.00 \mathrm{~J}$ & $4970.00 \mathrm{~J}$ & $4290.00 \mathrm{~J}$ \\
\hline Chroniun & 0.88 UJ & 0.87 UJ & $3.30 \mathrm{~J}$ & $3.70 \mathrm{~J}$ & $8.70 \mathrm{~J}$ & $1.70 \mathrm{~J}$ \\
\hline Cobalt & 0.66 UJ & $0.66 \mathrm{UJ}$ & $0.97 \mathrm{UJ}$ & $0.88 \mathrm{~J}$ & $2.80 \mathrm{UJ}$ & $0.73 \mathrm{UJ}$ \\
\hline Coppar & $9.20 \mathrm{~J}$ & $6.00 \mathrm{UJ}$ & $15.10 \mathrm{~J}$ & $12.40 \mathrm{~J}$ & $25.00 \mathrm{~d}$ & $8.50 \mathrm{~J}$ \\
\hline Iron & $32.30 \mathrm{~J}$ & $20.00 \mathrm{UJ}$ & $1530.00 \mathrm{~J}$ & $1380.00 \mathrm{~J}$ & $4920.00 \mathrm{~J}$ & $549.00 \mathrm{~J}$ \\
\hline Leed & $0.43 \mathrm{UJ}$ & $0.42 \mathrm{UJ}$ & $4.40 \mathrm{~J}$ & $2.30 \mathrm{~J}$ & $11.10 \mathrm{~J}$ & $0.57 \mathrm{~J}$ \\
\hline Mognes Iun & $1340.00 \mathrm{~J}$ & $1420.00 \mathrm{~J}$ & $2350.00 \mathrm{~J}$ & $1740.00 \mathrm{~J}$ & $3640.00 \mathrm{~J}$ & $2600.00 \mathrm{~J}$ \\
\hline Manganose & $21.70 \mathrm{~J}$ & $22.50 \mathrm{~J}$ & $107.00 \mathrm{~J}$ & $80.00 \mathrm{~J}$ & $254.00 \mathrm{~J}$ & $70.10 \mathrm{~J}$ \\
\hline Mercury & $0.10 \mathrm{UJ}$ & $0.11 \mathrm{uJ}$ & $0.09 \mathrm{UJ}$ & $0.10 \mathrm{UJ}$ & $0.10 \mathrm{UJ}$ & $0.10 \mathrm{UJ}$ \\
\hline Nickel & 0.88 UJ & $0.87 \mathrm{UJ}$ & $3.30 \mathrm{UJ}$ & $2.70 \mathrm{UJ}$ & $9.30 \mathrm{~J}$ & $1.70 \mathrm{uJ}$ \\
\hline Potassiun & $18000.00 \mathrm{~J}$ & $16000.00 \mathrm{~J}$ & $24000.00 \mathrm{~J}$ & $19600.00 \mathrm{~J}$ & $23800.00 \mathrm{~J}$ & $15900.00 \mathrm{~J}$ \\
\hline seleniun & 0.86 UJ & $0.84 \mathrm{UJ}$ & $4.30 \mathrm{UJ}$ & 0.87 UJ & $4.10 \mathrm{UJ}$ & $0.85 \mathrm{UJ}$ \\
\hline sllver & $0.44 \mathrm{UJ}$ & $0.44 \mathrm{UJ}$ & $0.42 \mathrm{UJ}$ & 0.43 UJ & $0.42 \mathrm{UJ}$ & $0.42 U \mathrm{~J}$ \\
\hline sodiun & $31.90 \mathrm{UJ}$ & $34.40 \mathrm{UJ}$ & $342.00 \mathrm{~J}$ & $233.00 \mathrm{~J}$ & $320.00 \mathrm{~J}$ & $49.60 \mathrm{~J}$ \\
\hline Thall lium & $3.20 \mathrm{R}$ & $3.20 R$ & $3.20 \mathrm{R}$ & $3.30 \mathrm{R}$ & $3.00 R$ & $3.20 \mathrm{R}$ \\
\hline Vanadiun & $0.44 \mathrm{UJ}$ & 0.44 Ud & $2.10 \mathrm{~J}$ & $2.60 \mathrm{~J}$ & $9.00 \mathrm{~J}$ & $0.63 \mathrm{~J}$ \\
\hline $2 \ln c$ & $39.10 \mathrm{~J}$ & $55.10 \mathrm{~J}$ & $131.00 \mathrm{~J}$ & $78.40 \mathrm{~J}$ & $271.00 \mathrm{~J}$ & $83.80 \mathrm{~J}$ \\
\hline Cyanlde & NR & NR & NR & NR & NR & MR \\
\hline Co-137 & $<1.140 \mathrm{UJ}$ & C.7342 UJ & $0.80 \mathrm{~J}$ & $1.489 \mathrm{UJ}$ & $<1.648$ UJ & $<.8158$ UJ \\
\hline $8 r=90$ & $-0.20 \mathrm{UJ}$ & 0.20 UJ & $0.00 \mathrm{UJ}$ & $-0.20 \mathrm{UJ}$ & -0.07 UJ & $-0.09 \mathrm{UJ}$ \\
\hline Tc. $\varphi$ & 0.20 UJ & $0.40 \mathrm{Ud}$ & $0.50 \mathrm{~J}$ & $0.60 \mathrm{~J}$ & $0.30 \mathrm{UJ}$ & $0.50 R$ \\
\hline
\end{tabular}

NOTE: All metals are reported in $\mathrm{mg} / \mathrm{kg}$. Radlonucl ldes are reported in pCl/g.

B - Anulyte found in assoclated blank as well as sample.

$\mathrm{J}$ - Not detected; aseociated value is estimated.

NR - Monreportable.

R - Dete are unusable.

$U$ - Not detected; value reported is sample quantitation IImit.

UJ - Not detected; may not accurately roflect sample quantitation $1 / \mathrm{mit}$. 
Table C-4. Reed Canarygrass Sample Results from 100-D Reactor Area Collected in 1991.

\begin{tabular}{|c|c|c|c|c|c|c|}
\hline \multirow{2}{*}{ Const I tuent } & \multicolumn{6}{|c|}{ semple Identification Numbers } \\
\hline & soovis & Boovwo & Boovw1 & BOOVN2 & 800 VW3 & Boonit \\
\hline Aluminum & 17.50 UJ & $53.00 \mathrm{~J}$ & $14.30 \mathrm{uJ}$ & $27.20 \mathrm{~J}$ & 13.40 US & $18.00 \mathrm{~J}$ \\
\hline Antimony & $1.60 \mathrm{UJ}$ & $1.70 \mathrm{UJ}$ & $6.70 \mathrm{UJ}$ & $6.90 \mathrm{uJ}$ & $6.30 \mathrm{UJ}$ & $6.60 \mathrm{UJ}$ \\
\hline Arsente & 0.43 UJ & $0.41 \mathrm{us}$ & 2.10 U.1 & $0.49 \mathrm{~J}$ & $0.44 \mathrm{UJ}$ & $0.43 \mathrm{UN}$ \\
\hline Bariun & $17.70 \mathrm{~J}$ & $23.50 \mathrm{~J}$ & $21.40 \mathrm{~J}$ & $28.40 \mathrm{~J}$ & $23.00 \mathrm{~J}$ & $28.00 \mathrm{~J}$ \\
\hline Beryllium & $0.20 \mathrm{UJ}$ & $0.21 \mathrm{UJ}$ & 0.22 UJ & $0.22 \mathrm{UJ}$ & $0.20 \mathrm{UJ}$ & 0.21 uJ \\
\hline Cectinlun & 0.20 Ud & $0.21 \mathrm{UJ}$ & $0.22 \mathrm{UJ}$ & $0.27 \mathrm{~J}$ & $0.20 \mathrm{UN}$ & $0.21 \mathrm{uJ}$ \\
\hline colclu & $2380.00 \mathrm{~J}$ & $5880.00 \mathrm{~d}$ & $4480.00 \mathrm{~J}$ & $5670.00 \mathrm{~d}$ & $5210.00 \mathrm{~J}$ & $5360.00 \mathrm{~J}$ \\
\hline Chromiun & 0.82 UJ & $1.00 \mathrm{~J}$ & $0.81 \mathrm{~J}$ & $1.30 \mathrm{~J}$ & $1.40 \mathrm{~J}$ & $1.60 \mathrm{~J}$ \\
\hline Cobalt & $0.61 \mathrm{UJ}$ & $0.63 \mathrm{UJ}$ & $0.65 \mathrm{UJ}$ & $0.67 \mathrm{UJ}$ & 0.61 UN & $0.64 \mathrm{us}$ \\
\hline Copper & $3.10 \mathrm{U}$ & $4.50 \mathrm{U}$ & $9.00 \mathrm{~J}$ & $4.80 \mathrm{~J}$ & $4.40 \mathrm{Ud}$ & $5.90 \mathrm{~J}$ \\
\hline Iron & $54.50 \mathrm{~J}$ & $119.00 \mathrm{~J}$ & $56.40 \mathrm{~J}$ & $80.50 \mathrm{~J}$ & $46.80 \mathrm{~J}$ & $82.90 \mathrm{~J}$ \\
\hline Lead & $0.63 \mathrm{UJ}$ & $0.41 \mathrm{uJ}$ & $0.43 \mathrm{UJ}$ & $0.44 \mathrm{Ud}$ & $0.46 \mathrm{UJ}$ & $0.43 \mathrm{UJ}$ \\
\hline Magnosicu & $1470.00 \mathrm{~J}$ & $2080.00 \mathrm{~J}$ & $2390.00 \mathrm{~J}$ & $2220.00 \mathrm{~J}$ & $2530.00 \mathrm{~J}$ & $2600.00 \mathrm{~J}$ \\
\hline Mangenese & $18.60 \mathrm{~J}$ & $26.20 \mathrm{~J}$ & $19.50 \mathrm{~J}$ & $16.90 \mathrm{~J}$ & $13.30 \mathrm{~J}$ & $10.50 \mathrm{~J}$ \\
\hline Mercury & $0.11 \mathrm{UJ}$ & $0.10 \mathrm{UJ}$ & $0.11 \mathrm{uJ}$ & $0.11 \mathrm{uJ}$ & $0.10 \mathrm{UJ}$ & $0.11 \mathrm{us}$ \\
\hline Nickel & 0.82 Ud & $1.20 \mathrm{UJ}$ & $0.87 \mathrm{UJ}$ & 0.89 UJ & $0.81 \mathrm{UJ}$ & $0.85 \mathrm{UJ}$ \\
\hline Potaselum & $15400.00 \mathrm{~J}$ & $15300.00 \mathrm{~J}$ & $26000.00 \mathrm{~J}$ & $15500.00 \mathrm{~J}$ & $12900.00 \mathrm{~d}$ & 21100.00 \\
\hline selentum & 0.86 UJ & $0.83 \mathrm{UJ}$ & $4.30 \mathrm{UJ}$ & $0.87 \mathrm{UJ}$ & $0.87 \mathrm{UJ}$ & $0.86 \mathrm{UJ}$ \\
\hline silver & $0.61 \mathrm{UJ}$ & $0.42 \mathrm{UJ}$ & $1.10 \mathrm{UJ}$ & $1.10 \mathrm{UJ}$ & $1.00 \mathrm{UJ}$ & $1.10 \mathrm{uJ}$ \\
\hline sodiun & $43.70 \mathrm{UJ}$ & $45.30 \mathrm{UJ}$ & $40.80 \mathrm{~J}$ & $42.10 \mathrm{~J}$ & $41.60 \mathrm{~J}$ & $39.60 \mathrm{~J}$ \\
\hline Thallium & $3.20 R$ & $3.10 R$ & $3.20 \mathrm{uJ}$ & $3.30 \mathrm{uJ}$ & $3.30 \mathrm{UJ}$ & $3.20 \mathrm{UJ}$ \\
\hline Vanadium & $0.41 \mathrm{UJ}$ & $0.42 \mathrm{UJ}$ & $0.65 \mathrm{UJ}$ & $0.67 \mathrm{UJ}$ & $0.61 \mathrm{UJ}$ & $0.64 \mathrm{UJ}$ \\
\hline 2 inc & $64.90 \mathrm{~J}$ & $64.20 \mathrm{~J}$ & $60.20 \mathrm{~J}$ & $36.90 \mathrm{~J}$ & $90.30 \mathrm{~J}$ & $68.80 \mathrm{~J}$ \\
\hline Cranide & NR & NR & NR & NR & NR & NR \\
\hline$C 8-137$ & $=1.087 \mathrm{UJ}$ & $<1.190$ UJ &. .748 UJ & $<1.504$ UJ & $<.909$ UJ & $<1.064$ UJ \\
\hline $5 r \cdot 90$ & $-0.40 \mathrm{UJ}$ & $0.00 \mathrm{UJ}$ & $0.00 \mathrm{UJ}$ & $-0.50 \mathrm{UJ}$ & $0.00 \mathrm{UJ}$ & -0.05 UJ \\
\hline Te.99 & $0.20 \mathrm{UJ}$ & $0.30 \mathrm{~J}$ & $0.20 \mathrm{~J}$ & $0.20 \mathrm{UJ}$ & $0.16 \mathrm{~J}$ & $0.40 \mathrm{~J}$ \\
\hline
\end{tabular}

NOTE: Al! matals are reported in $\mathrm{mg} / \mathrm{kg}$. Radlonuclides are reported in $\mathrm{pC} 1 / \mathrm{s}$.

B - Analyte found in associated blank as well as sample.

$J$. Not detected; associ feted value is estimated.

NR - Nonreportable.

$R$ - Data are unusable.

$U$ - Not detected; value reported is semple quantitation IImit.

UJ - Not detected; may not eccurately reflect sample quantitation limit. 
Table C-5. Reed Canarygrass Duplicate Sample Results from 100-D Reactor Area Collected in 1991.

\begin{tabular}{|c|c|c|c|c|c|c|}
\hline \multirow{2}{*}{ Const i tuent } & \multicolumn{6}{|c|}{ Sample Identification Numbers } \\
\hline & 801047 & B01048 & B01049 & B01050 & 801051 & B01052 \\
\hline Aluminum & $26.40 \mathrm{~J}$ & $19.70 \mathrm{~J}$ & $15.00 \mathrm{UJ}$ & $13.90 \mathrm{~J}$ & $13.40 \mathrm{UJ}$ & $13.30 \mathrm{UJ}$ \\
\hline Ant imony & $6.50 \mathrm{UJ}$ & $7.30 \mathrm{UJ}$ & $7.00 \mathrm{UJ}$ & $6.40 \mathrm{UJ}$ & $6.30 \mathrm{UJ}$ & $6.20 \mathrm{UJ}$ \\
\hline Arsenic & $0.41 \mathrm{UJ}$ & $0.46 \mathrm{UJ}$ & 0.47 UJ & $0.43 \mathrm{UJ}$ & 0.48 UJ & $0.40 \mathrm{UJ}$ \\
\hline Barium & $22.10 \mathrm{~J}$ & $33.50 \mathrm{~J}$ & $21.00 \mathrm{~J}$ & $14.50 \mathrm{~J}$ & $19.60 \mathrm{~J}$ & $31.50 \mathrm{~J}$ \\
\hline Beryllium & $0.21 U_{11}$ & $0.24 \mathrm{UJ}$ & $0.23 \mathrm{UJ}$ & $0.21 \mathrm{UJ}$ & $0.20 \mathrm{UJ}$ & $0.20 \mathrm{UJ}$ \\
\hline Cacmium & $0.21 \mathrm{UJ}$ & $0.24 \mathrm{UJ}$ & $0.23 \mathrm{UJ}$ & $0.21 \mathrm{UJ}$ & $0.20 \mathrm{UJ}$ & $0.25 \mathrm{UJ}$ \\
\hline Calcium & $3570.00 \mathrm{~J}$ & $6230.00 \mathrm{~J}$ & $3370.00 \mathrm{~J}$ & $2940.00 \mathrm{~J}$ & $4900.00 \mathrm{~J}$ & $5800.00 \mathrm{~J}$ \\
\hline Chromiun & $0.68 \downarrow$ & $1.00 \mathrm{~J}$ & $0.64 \mathrm{~J}$ & $0.41 \mathrm{UJ}$ & $1.30 \mathrm{UJ}$ & $1.70 \mathrm{UJ}$ \\
\hline Cobalt & 0.62 UJ & $0.71 \mathrm{us}$ & $0.68 \mathrm{UJ}$ & 0.62 UJ & $0.61 \mathrm{UJ}$ & $0.60 \mathrm{UJ}$ \\
\hline Copper & $3.20 \mathrm{~J}$ & $3.30 \mathrm{~J}$ & $3.60 \mathrm{~J}$ & $2.90 \mathrm{~J}$ & 8.50 UJ & $6.70 \mathrm{UJ}$ \\
\hline Iron & $70.00 \mathrm{~J}$ & $47.70 \mathrm{~J}$ & $35.60 \mathrm{~J}$ & 34.40 .1 & $42.00 \mathrm{~J}$ & $40.30 \mathrm{~J}$ \\
\hline Lead & $0.41 \mathrm{UJ}$ & $0.46 \mathrm{UJ}$ & $0.47 \mathrm{UJ}$ & $0.43 \mathrm{UJ}$ & 0.37 UJ & $0.68 \mathrm{UJ}$ \\
\hline Magnes ium & $1670.00 \mathrm{~J}$ & $2230.00 \mathrm{~J}$ & $1260.00 \mathrm{~J}$ & $1410.00 \mathrm{~J}$ & $2710.00 \mathrm{~J}$ & $2810.00 \mathrm{~J}$ \\
\hline Manganese & $29.90 \mathrm{~J}$ & $16.50 \mathrm{~J}$ & $12.50 \mathrm{~J}$ & $13.20 \mathrm{~J}$ & $17.10 \mathrm{~J}$ & $15.30 \mathrm{~J}$ \\
\hline Mercury & $0.10 \mathrm{UJ}$ & $0.10 \mathrm{UJ}$ & $0.11 \mathrm{UJ}$ & $0.11 \mathrm{UJ}$ & $0.10 \mathrm{UJ}$ & $0.10 \mathrm{UJ}$ \\
\hline Nickel & $0.83 \mathrm{UJ}$ & $0.95 \mathrm{UJ}$. & $0.91 \mathrm{UJ}$ & $1.20 \mathrm{~J}$ & $0.81 \mathrm{UJ}$ & $1.10 \mathrm{~J}$ \\
\hline Potassium & $18300.00 \mathrm{~J}$ & $16700.00 \mathrm{~J}$ & $21100.00 \mathrm{~J}$ & $14800.00 \mathrm{~J}$ & $19300.00 \mathrm{~J}$ & $18500.00 \mathrm{~J}$ \\
\hline Selenium & 0.82 UJ & $0.92 \mathrm{UJ}$ & $0.93 \mathrm{UJ}$ & $0.87 \mathrm{UJ}$ & $4.20 \mathrm{UJ}$ & $0.80 \mathrm{UJ}$ \\
\hline Silver & $1.00 \mathrm{UJ}$ & $1.20 \mathrm{UJ}$ & $1.10 \mathrm{UJ}$ & $1.00 \mathrm{UJ}$ & $1.00 \mathrm{UJ}$ & $1.00 \mathrm{UJ}$ \\
\hline Sodium & $40.80 \mathrm{~J}$ & $25.00 \mathrm{~J}$ & $38.10 \mathrm{~J}$ & $14.50 \mathrm{~J}$ & $25.70 \mathrm{UJ}$ & $32.70 \mathrm{UJ}$ \\
\hline Thallium & $3.10 \mathrm{UJ}$ & $3.50 \mathrm{UJ}$ & $3.50 \mathrm{UJ}$ & $3.30 \mathrm{UJ}$ & $0.42 \mathrm{UJ}$ & $0.40 \mathrm{UJ}$ \\
\hline Vanadium & $0.62 \mathrm{UJ}$ & $0.71 \mathrm{UJ}$ & $0.68 \mathrm{UJ}$ & 0.62 UJ & 0.61 UJ & $0.60 \mathrm{UJ}$ \\
\hline 2 inc & $34.60 \mathrm{~J}$ & $168.00 \mathrm{~J}$ & $133.00 \mathrm{~J}$ & $85.20 \mathrm{~J}$ & $90.50 \mathrm{~J}$ & $87.20 \mathrm{~J}$ \\
\hline Cyanide & NR & NR & NR & NR & NR & NR \\
\hline Cs- 137 & $<.7619$ UJ & $<1.315 \mathrm{UJ}$ & $<.9526 \mathrm{UJ}$ & $<.6895$ UJ & $<1.195$ UJ & $<.6638 \mathrm{UJ}$ \\
\hline Sr. .00 & -2.00 Ud & $0.10 \mathrm{~J}$ & $-1.00 \mathrm{UJ}$ & $-0.2 \mathrm{UJ}$ & $0.60 \mathrm{~J}$ & $-0.90 \mathrm{UJ}$ \\
\hline Tc- 99 & $0.13 \mathrm{~J}$ & $0.30 \mathrm{~J}$ & $0.30 R$ & $0.76 \mathrm{~J}$ & $0.20 \mathrm{UJ}$ & $0.20 \mathrm{UJ}$ \\
\hline
\end{tabular}

NOTE: All metals are reported in $\mathrm{mg} / \mathrm{kg}$. Radionuclides are reported in $\mathrm{PCi} / \mathrm{g}$.

$B=$ Analyte found in associated blank as well as sample.

$J=$ Not detected; associated value is estimated.

NR $=$ Nonreportable.

$R=$ Data are unusable.

$U=$ Not detected; value reported is sample quantitation limit

$U J=$ Not detected; may not accurately reflect sample quantitation limit. 
Table C-6. Reed Canarygrass Sample Resulis Collected Below 100-D Reactor in 1991.

\begin{tabular}{|c|c|c|c|c|c|c|}
\hline \multirow{2}{*}{ Const i tuent } & \multicolumn{6}{|c|}{ Sample Identification Numbers } \\
\hline & Boovu7 & BOOVW8 & BoOVW9 & BOOVXO & BoOVX1 & $800 \mathrm{~V} \times 2$ \\
\hline Aluminum & $19.90 \mathrm{UJ}$ & $193.00 \mathrm{~J}$ & $27.90 \mathrm{UJ}$ & $165.00 \mathrm{~J}$ & $36.10 \mathrm{~J}$ & $168.00 \mathrm{~J}$ \\
\hline Ant imony & $1.60 \mathrm{UJ}$ & $1.60 \mathrm{UJ}$ & $1.90 \mathrm{UJ}$ & $1.70 \mathrm{us}$ & $1.70 \mathrm{uJ}$ & 1.70 UJ \\
\hline Arsenic & $0.44 \mathrm{~J}$ & $0.51 \mathrm{~J}$ & $0.49 \mathrm{UJ}$ & $0.56 \mathrm{~J}$ & $0.43 \mathrm{uJ}$ & $0.41 \mathrm{UJ}$ \\
\hline Barium & $10.20 \mathrm{~J}$ & $19.50 \mathrm{~J}$ & $14.60 \mathrm{~J}$ & $14.10 \mathrm{~J}$ & $11.60 \mathrm{~J}$ & $20.90 \mathrm{~J}$ \\
\hline Beryllium & $0.20 \mathrm{UJ}$ & $0.21 \mathrm{UJ}$ & 0.23 UJ & 0.21 us & $0.21 \mathrm{uJ}$ & $0.21 \mathrm{UJ}$ \\
\hline Cacmium & $0.20 \mathrm{UJ}$ & $0.21 \mathrm{UJ}$ & 0.23 UJ & $0.29 \mathrm{UJ}$ & $0.21 \mathrm{UJ}$ & $0.21 \mathrm{UJ}$ \\
\hline Calcium & $2450.00 \mathrm{~J}$ & $3450.00 \mathrm{~J}$ & $3150.00 \mathrm{~J}$ & $3180.00 \mathrm{~J}$ & $4030.00 \mathrm{~J}$ & $4860.00 \mathrm{~J}$ \\
\hline Chromiun & $0.82 \mathrm{UJ}$ & $0.82 \mathrm{UJ}$ & 0.93 Ud & $1.50 \mathrm{~J}$ & $1.50 \mathrm{~J}$ & $1.80 \mathrm{~J}$ \\
\hline Cobalt & $0.61 \mathrm{UJ}$ & $1.20 \mathrm{UJ}$ & $0.69 \mathrm{UJ}$ & $0.98 \mathrm{UJ}$ & $0.63 \mathrm{UJ}$ & $0.64 \mathrm{UJ}$ \\
\hline Copper & $3.30 \mathrm{UJ}$ & $7.90 \mathrm{~J}$ & $6.70 \mathrm{UJ}$ & $5.80 \mathrm{UJ}$ & $3.40 \mathrm{UJ}$ & $5.40 \mathrm{UJ}$ \\
\hline Iron & $46.70 \mathrm{~J}$ & $355.00 \mathrm{~J}$ & $66.70 \mathrm{~J}$ & $286.00 \mathrm{~J}$ & $82.10 \mathrm{~J}$ & $285.00 \mathrm{~J}$ \\
\hline Lead & $2.20 \mathrm{UJ}$ & $0.61 \mathrm{~J}$ & $2.40 \mathrm{UJ}$ & $0.56 \mathrm{~J}$ & $0.43 \mathrm{UJ}$ & $0.41 \mathrm{UJ}$ \\
\hline Magnesium & $1230.00 \mathrm{~J}$ & $1970.00 \mathrm{~J}$ & $1650.00 \mathrm{~J}$ & $1440.00 \mathrm{~J}$ & $1750.00 \mathrm{~J}$ & $2350.00 \mathrm{~J}$ \\
\hline Manganese & $17.90 \mathrm{~J}$ & $80.50 \mathrm{~J}$ & $36.60 \mathrm{~J}$ & $36.60 \mathrm{~J}$ & $23.60 \mathrm{~J}$ & $44.70 \mathrm{~J}$ \\
\hline Mercury & 0.09 Us & $0.10 \mathrm{UJ}$ & $0.11 \mathrm{uJ}$ & $0.09 \mathrm{UJ}$ & $0.10 \mathrm{UJ}$ & $0.10 \mathrm{UJ}$ \\
\hline Nickel & $0.82 \mathrm{UJ}$ & $1.80 \mathrm{UJ}$ & $0.93 \mathrm{UJ}$ & $1.60 \mathrm{UJ}$ & $0.84 \mathrm{UJ}$ & $0.85 \mathrm{UJ}$ \\
\hline Potassium & $13500.00 \mathrm{~J}$ & $19200.00 \mathrm{~J}$ & $12800.00 \mathrm{~J}$ & $12100.00 \mathrm{~J}$ & $13500.00 \mathrm{~J}$ & $16800.00 \mathrm{~J}$ \\
\hline seleniun & $0.87 \mathrm{UJ}$ & $0.88 \mathrm{UJ}$ & $0.97 \mathrm{UJ}$ & $0.86 \mathrm{UJ}$ & $0.86 \mathrm{UJ}$ & 0.82 UJ \\
\hline silver & $0.41 \mathrm{UJ}$ & $0.41 \mathrm{UJ}$ & $0.46 \mathrm{UJ}$ & $0.42 \mathrm{UJ}$ & $0.42 \mathrm{UJ}$ & $0.42 \mathrm{UJ}$ \\
\hline sodiun & $42.80 \mathrm{UJ}$ & $70.80 \mathrm{~J}$ & $102.00 \mathrm{~J}$ & $96.30 \mathrm{~J}$ & $80.80 \mathrm{~J}$ & $57.90 \mathrm{~J}$ \\
\hline Thallium & $3.30 \mathrm{R}$ & $3.30 \mathrm{R}$ & $3.60 \mathrm{R}$ & $3.20 R$ & $3.20 \mathrm{R}$ & $3.10 \mathrm{R}$ \\
\hline Vanadium & $0.41 \mathrm{UJ}$ & $0.41 \mathrm{UJ}$ & $0.46 \mathrm{UJ}$ & $0.42 \mathrm{UJ}$ & $0.42 \mathrm{UJ}$ & $0.42 \mathrm{UJ}$ \\
\hline $2 \ln c$ & $24.00 \mathrm{~J}$ & $58.60 \mathrm{~J}$ & $28.10 \mathrm{~J}$ & $35.80 \mathrm{~J}$ & $20.90 \mathrm{~J}$ & $44.80 \mathrm{~J}$ \\
\hline Cyanto & NR & MR & NR & NR & NR & NR \\
\hline Cs- 137 & $<.9632 \mathrm{~J}$ & $<1.974$ UJ & $<1.079$ UJ & $<1.187 \mathrm{UJ}$ & $<.9326 \mathrm{UJ}$ & $<1.737 \mathrm{UJ}$ \\
\hline Sr-90 & $0.08 \mathrm{UJ}$ & $-0.40 \mathrm{UJ}$ & $-0.10 \mathrm{Ud}$ & $0.00 \mathrm{UJ}$ & -0.03 UJ & $=0.30 \mathrm{UJ}$ \\
\hline Tc-99 & $-0.10 R$ & $0.30 \mathrm{R}$ & $0.90 R$ & $0.20 \mathrm{UJ}$ & $0.40 \mathrm{~J}$ & $0.20 \mathrm{UJ}$ \\
\hline
\end{tabular}

NOTE: All metals are reported in $\mathrm{mg} / \mathrm{kg}$. Radionuclides are reported in pCl/g.

B = Analyte found in essociated blank as well as sample.

$J$ - Not detected; assoclated value is estimated.

NR - Nonreportable.

$R$ " Data are unusable.

$U$ - Not detected; value reported is sample quantitation 1 imlt.

UJ - Not detected; may not accurately reflect sample quantitation limit. 
Table C-7. Reed Canarygrass Sample Results from 100-BC Reactor Area Collected Downriver in 1991.

\begin{tabular}{|c|c|c|c|c|c|c|}
\hline \multirow{2}{*}{ Const ituent } & \multicolumn{6}{|c|}{ Sample Identification Numbers } \\
\hline & Boov1 & BooW2 & Boovv3 & 800VV4 & BooW5 & BooVv6 \\
\hline Aluminum & $189.00 \mathrm{~J}$ & $240.00 \mathrm{~J}$ & $206.00 \mathrm{~J}$ & $447.00 \mathrm{~J}$ & $195.00 \mathrm{~J}$ & $29.20 \mathrm{~J}$ \\
\hline Ant imony & $6.60 \mathrm{UJ}$ & $6.30 \mathrm{UJ}$ & $6.40 \mathrm{UJ}$ & $6.40 \mathrm{UJ}$ & $6.20 \mathrm{UJ}$ & $6.60 \mathrm{UJ}$ \\
\hline Arsenic & $1.10 \mathrm{UJ}$ & $0.88 \mathrm{UJ}$ & 0.59 UJ & $0.40 \mathrm{UJ}$ & $0.66 \mathrm{UJ}$ & 0.57 UJ \\
\hline Barium & $18.10 \mathrm{~J}$ & $35.60 \mathrm{~J}$ & $16.50 \mathrm{~J}$ & $34.10 \mathrm{~J}$ & $40.30 \mathrm{~J}$ & $24.20 \mathrm{~J}$ \\
\hline Beryllium & $0.21 \mathrm{UJ}$ & $0.20 \mathrm{UJ}$ & 0.21 UJ & $0.20 \mathrm{UJ}$ & $0.20 \mathrm{UJ}$ & $0.21 \mathrm{UJ}$ \\
\hline Cacmium & $0.21 \mathrm{UJ}$ & $0.20 \mathrm{UJ}$ & $0.21 \mathrm{UJ}$ & $0.20 \mathrm{UJ}$ & $0.20 \mathrm{UJ}$ & $0.21 \mathrm{UJ}$ \\
\hline Calcium & $4060.00 \mathrm{~J}$ & $6190.00 \mathrm{~J}$ & $3140.00 \mathrm{~J}$ & $4840.00 \mathrm{~J}$ & $4200.00 \mathrm{~J}$ & $4180.00 \mathrm{~J}$ \\
\hline Chromiun & $1.60 \mathrm{UJ}$ & $1.40 \mathrm{UJ}$ & $1.70 \mathrm{UJ}$ & $1.80 \mathrm{U}$ & $1.80 \mathrm{UJ}$ & $0.89 \mathrm{UJ}$ \\
\hline Cobalt & $0.64 \mathrm{UJ}$ & $0.61 \mathrm{UJ}$ & 0.62 UJ & $0.72 \mathrm{~J}$ & $0.60 \mathrm{UJ}$ & 0.64 UJ \\
\hline Copper & $6.90 \mathrm{UJ}$ & $8.10 \mathrm{UJ}$ & $9.10 \mathrm{UJ}$ & $9.60 \mathrm{U}$ & $6.80 \mathrm{UJ}$ & $4.90 \mathrm{UJ}$ \\
\hline Iron & $351.00 \mathrm{~J}$ & $421.00 \mathrm{~J}$ & $415.00 \mathrm{~J}$ & $842.00 \mathrm{~J}$ & $380.00 \mathrm{~J}$ & $84.20 \mathrm{~J}$ \\
\hline Lead & $1.10 \mathrm{uJ}$ & $0.86 \mathrm{UJ}$ & $1.40 \mathrm{~J}$ & $1.70 \mathrm{~J}$ & $1.00 \mathrm{UJ}$ & $0.21 \mathrm{uJ}$ \\
\hline Magnesium & $2190.00 \mathrm{~J}$ & $3190.00 \mathrm{~J}$ & $1760.00 \mathrm{~J}$ & $2130.00 \mathrm{~J}$ & $1570.00 \mathrm{~J}$ & $1880.00 \mathrm{~J}$ \\
\hline Manganese & $49.00 \mathrm{~J}$ & $76.20 \mathrm{~J}$ & $79.90 \mathrm{~J}$ & $83.20 \mathrm{~J}$ & $33.60 \mathrm{~J}$ & $40.90 \mathrm{~J}$ \\
\hline Mercury & 0.36 UJ & $0.10 \mathrm{UJ}$ & $0.10 \mathrm{UJ}$ & $0.10 \mathrm{U.J}$ & $0.10 \mathrm{UJ}$ & $0.10 \mathrm{UJ}$ \\
\hline Nickel & $0.85 \mathrm{UJ}$ & $1.50 \mathrm{~J}$ & $0.82 \mathrm{UJ}$ & $1.50 \mathrm{~J}$ & $0.83 \mathrm{~J}$ & $0.86 \mathrm{UJ}$ \\
\hline Potassium & $14700.00 \mathrm{~J}$ & $18800.00 \mathrm{~J}$ & $21700.00 \mathrm{~J}$ & $17600.00 \mathrm{~J}$ & $15400.00 \mathrm{~J}$ & $15800.00 \mathrm{~J}$ \\
\hline Seleniun & 0.87 UJ & $0.82 \mathrm{UJ}$ & $4.20 \mathrm{UJ}$ & $0.80 \mathrm{UJ}$ & $4.20 \mathrm{UJ}$ & 0.85 UJ \\
\hline silver & $1.10 \mathrm{UJ}$ & $1.00 \mathrm{UJ}$ & $1.00 \mathrm{UJ}$ & $1.00 \mathrm{UJ}$ & 0.99 UJ & $1.10 \mathrm{UJ}$ \\
\hline Sodium & $40.40 \mathrm{UJ}$ & 70.80 UJ & $95.40 \mathrm{~J}$ & $112.00 \mathrm{~J}$ & $39.20 \mathrm{UJ}$ & $17.50 \mathrm{UJ}$ \\
\hline Thallium & $0.43 \mathrm{UJ}$ & $0.41 \mathrm{UJ}$ & $0.42 \mathrm{UJ}$ & $0.40 \mathrm{UJ}$ & $0.42 \mathrm{UJ}$ & $0.42 \mathrm{UJ}$ \\
\hline Vanadium & $0.64 \mathrm{UJ}$ & $1.10 \mathrm{~J}$ & $0.98 \mathrm{~J}$ & $1.60 \mathrm{~J}$ & $0.85 \mathrm{~J}$ & $0.64 \mathrm{UJ}$ \\
\hline Zine & $50.00 \mathrm{~J}$ & $54.70 \mathrm{~J}$ & $101.00 \mathrm{~J}$ & $87.90 \mathrm{~J}$ & $41.00 \mathrm{~J}$ & $63.70 \mathrm{~J}$ \\
\hline Cyanide & MR & MR & NR & NR & NR & MR \\
\hline$C_{8}-137$ & $<1.432 \mathrm{UJ}$ & $<1.459$ UJ & $<1.121 \mathrm{UJ}$ & $<.863$ UJ & $<1.204$ UJ & $<.6650$ UJ \\
\hline$S r-90$ & $-2.00 \mathrm{UJ}$ & $0.01 \mathrm{UJ}$ & $0.06 \mathrm{UJ}$ & $0.20 \mathrm{UJ}$ & $0.06 \mathrm{UJ}$ & -0.50 UJ \\
\hline TC. 99 & $0.30 \mathrm{~J}$ & $0.40 \mathrm{~J}$ & $0.10 \mathrm{UJ}$ & $0.10 \mathrm{UJ}$ & $0.10 \mathrm{UJ}$ & $0.20 \mathrm{UJ}$ \\
\hline
\end{tabular}

NOTE: All metals are reported in $\mathrm{mg} / \mathrm{kg}$. Radionuclides are reported in $\mathrm{pCi} / \mathrm{g}$.

$B=$ Analyte found in associated blank as well as sample.

$J$ = Not detected; associated value is estimated.

NR = Monreportable.

$R$ = Data are unusable.

$U$ = Mot detected; value reported is sample quantitation limit.

UJ = Not detected; may not accurately reflect sample quantitation limit. 
Table C-8. Reed Canarygrass Control Sample Results from 1991.

\begin{tabular}{|c|c|c|c|c|c|c|}
\hline \multirow{2}{*}{ Constituent } & \multicolumn{6}{|c|}{ Sample Identification Numbers } \\
\hline & Boovr3 & BoovTh & BOOVT5 & BoOVT6 & BoovT7 & BoOVT8 \\
\hline Aluninum & $1290.00 \mathrm{~J}$ & $480.00 \mathrm{~J}$ & $1950.00 \mathrm{~d}$ & $3260.00 \mathrm{~J}$ & $779.00 \mathrm{~J}$ & $847.00 \mathrm{~J}$ \\
\hline Ant imony & 6.70 UJ & $6.60 \mathrm{UJ}$ & $6.30 \mathrm{UJ}$ & $6.50 \mathrm{UJ}$ & $6.20 \mathrm{UJ}$ & $6.60 \mathrm{UJ}$ \\
\hline Arsenic & 0.93 UJ & 0.93 UJ & $3.00 \mathrm{UJ}$ & $4.20 \mathrm{UJ}$ & 0.84 UJ & $1.30 \mathrm{UJ}$ \\
\hline Barium & $42.70 \mathrm{~J}$ & $21.60 \mathrm{~J}$ & $48.20 \mathrm{~J}$ & $55.90 \mathrm{~J}$ & $30.90 \mathrm{~J}$ & $31.20 \mathrm{~J}$ \\
\hline Beryllium & 0.21 UJ & $0.21 \mathrm{UJ}$ & $0.20 \mathrm{UJ}$ & 0.21 UJ & $0.20 \mathrm{UJ}$ & $0.21 \mathrm{UJ}$ \\
\hline Cactmiun & 0.54 Ud & 0.21 Ud & $0.74 \mathrm{UJ}$ & $0.87 U$ & $0.41 \mathrm{uJ}$ & $0.42 \mathrm{UJ}$ \\
\hline Calcium & $5650.00 \mathrm{~J}$ & $4050.00 \mathrm{~J}$ & $5300.00 \mathrm{~J}$ & $6320.00 \mathrm{~J}$ & $5620.00 \mathrm{~J}$ & $5150.00 \mathrm{~J}$ \\
\hline Chromiun & $4.50 \mathrm{~J}$ & $2.20 \mathrm{UJ}$ & $5.40 \mathrm{~J}$ & $8.70 \mathrm{~J}$ & $3.20 \mathrm{UJ}$ & $3.40 \mathrm{UJ}$ \\
\hline Cobalt & $0.64 \mathrm{UJ}$ & $0.64 \mathrm{UJ}$ & $1.30 \mathrm{~J}$ & $2.60 \mathrm{~J}$ & $0.60 \mathrm{~J}$ & 0.64 UJ \\
\hline Copper & $13.30 \mathrm{~J}$ & $10.90 \mathrm{~J}$ & $14.80 \mathrm{~J}$ & $19.90 \mathrm{~J}$ & $12.40 \mathrm{~J}$ & $15.10 \mathrm{~J}$ \\
\hline Iron & $2190.00 \mathrm{~J}$ & $903.00 \mathrm{~J}$ & $3330.00 \mathrm{~J}$ & $5550.00 \mathrm{~J}$ & $1360.00 \mathrm{~J}$ & $1520.00 \mathrm{~J}$ \\
\hline Lend & $3.60 \mathrm{~J}$ & $2.40 \mathrm{~J}$ & $6.80 \mathrm{~J}$ & $0.40 \mathrm{UJ}$ & 0.29 UJ & $1.40 \mathrm{~J}$ \\
\hline Magnesium & $2330.00 \mathrm{~d}$ & $2110.00 \mathrm{~J}$ & $4260.00 \mathrm{~J}$ & $4430.00 \mathrm{~J}$ & $2680.00 \mathrm{~J}$ & $3470.00 \mathrm{~J}$ \\
\hline Manganese & $107.00 \mathrm{~J}$ & $68.00 \mathrm{~J}$ & $135.00 \mathrm{~J}$ & $256.00 \mathrm{~J}$ & $70.30 \mathrm{~J}$ & $79.20 \mathrm{~J}$ \\
\hline Mercury & $0.10 \mathrm{UJ}$ & $0.10 \mathrm{UJ}$ & $0.10 \mathrm{UJ}$ & $1.10 \mathrm{UJ}$ & $0.09 \mathrm{UJ}$ & $0.10 \mathrm{UJ}$ \\
\hline Nickel & $3.90 \mathrm{~J}$ & $1.50 \mathrm{~J}$ & $4.90 \mathrm{~J}$ & $9.10 \mathrm{~J}$ & $2.10 \mathrm{~J}$ & $2.70 \mathrm{~J}$ \\
\hline Potassium & $14900.00 \mathrm{~J}$ & $16300.00 \mathrm{~J}$ & $26400.00 \mathrm{~J}$ & $24300.00 \mathrm{~J}$ & $22200.00 \mathrm{~J}$ & $30700.00 \mathrm{~J}$ \\
\hline Selenium & $4.20 \mathrm{UJ}$ & 0.86 UJ & $0.81 \mathrm{UJ}$ & $4.20 \mathrm{UJ}$ & $4.00 \mathrm{UJ}$ & 0.83 UJ \\
\hline silver & $1.10 \mathrm{UJ}$ & $1.10 \mathrm{UJ}$ & $1.00 \mathrm{UJ}$ & $1.00 \mathrm{UJ}$ & $1.00 \mathrm{UJ}$ & $1.10 \mathrm{UJ}$ \\
\hline sodium & $160.00 \mathrm{~J}$ & $82.50 \mathrm{~J}$ & $537.00 \mathrm{~J}$ & $505.00 \mathrm{~J}$ & $127.00 \mathrm{~J}$ & $301.00 \mathrm{~J}$ \\
\hline Thallium & $0.42 \mathrm{UJ}$ & $0.43 \mathrm{UJ}$ & $0.41 \mathrm{uJ}$ & $0.42 \mathrm{UJ}$ & $0.40 \mathrm{UJ}$ & 0.41 UJ \\
\hline Vanadiun & $3.80 \mathrm{~J}$ & $1.60 \downarrow$ & $5.80 \mathrm{~J}$ & $10.20 \mathrm{~J}$ & $2.70 \mathrm{~J}$ & $2.60 \mathrm{~J}$ \\
\hline 2 inc & $90.20 \mathrm{~J}$ & $70.70 \mathrm{~J}$ & $97.70 \mathrm{~J}$ & $135.00 \mathrm{~J}$ & $114.00 \mathrm{~J}$ & $136.00 \mathrm{~J}$ \\
\hline Cyanide & NR & NR & NR & MR & NR & NR \\
\hline $\mathrm{Cs} \cdot 137$ & $<.954$ Ud & $<1.804$ US & $<1.138 \mathrm{UJ}$ & $<.896$ UJ & $<1.463 \mathrm{UJ}$ & $<1.63 \mathrm{UJ}$ \\
\hline $5 r \cdot 90$ & $0.09 \mathrm{UJ}$ & $0.01 \mathrm{UJ}$ & 0.04 UJ & $0.80 \mathrm{uJ}$ & $-0.50 \mathrm{UJ}$ & $0.01 \mathrm{UJ}$ \\
\hline $16-99$ & $0.20 \mathrm{UJ}$ & $0.30 \mathrm{UJ}$ & $0.30 \mathrm{~J}$ & $0.10 \mathrm{UJ}$ & $0.30 \mathrm{UJ}$ & $0.10 \mathrm{UJ}$ \\
\hline
\end{tabular}

NOTE: All motals are reported in $\mathrm{mg} / \mathrm{kg}$. Radionuclides are roported in $\mathrm{pcl} / \mathrm{g}$.

B - Analyte found in associated blank as well as semple.

$\mathrm{J}$ - Not datected; associated value is estimated.

NR = Nonreportable.

$R$ - Date are unusable.

$U$. Not detected; value reported is sample quantitation I imit.

UJ a Not detected; may not accurately reflect sample quantitation IImit. 
Table C-9. Reed Canarygrass Sample Results from 100-F Reactor Area Collected Downriver and Upriver in 1992.

\begin{tabular}{|c|c|c|c|c|c|c|}
\hline \multirow{2}{*}{ Const i tuent } & \multicolumn{6}{|c|}{ Sample Identification Numbers } \\
\hline & $806 N 63$ & $806 N 64$ & B06N65 & B06N66 & $806 N 67$ & BOSN68 \\
\hline Aluninum & 142.00 & 252.00 & $46.50 \mathrm{~B}$ & 189.00 & 339.00 & 608.00 \\
\hline Ant imony & $5.90 \mathrm{U}$ & $4.20 \mathrm{U}$ & $5.60 \mathrm{U}$ & $7.20 \mathrm{U}$ & $4.30 \mathrm{U}$ & $4.70 \mathrm{U}$ \\
\hline Arsenic & $1.00 \mathrm{U}$ & $0.72 U$ & $0.96 \mathrm{U}$ & $1.10 \mathrm{U}$ & $0.90 \mathrm{~B}$ & $1.00 \mathrm{~B}$ \\
\hline Barlum & $22.00 \mathrm{~B}$ & $19.60 \mathrm{~B}$ & $16.50 \mathrm{~B}$ & $26.50 \mathrm{~B}$ & $23.90 \mathrm{~B}$ & $23.10 \mathrm{~B}$ \\
\hline Beryllium & $0.14 \mathrm{U}$ & $0.10 u$ & $0.13 \mathrm{U}$ & $0.17 \mathrm{U}$ & $0.10 \mathrm{U}$ & $0.54 \mathrm{U}$ \\
\hline Cadmilum & $0.35 \mathrm{U}$ & $0.25 \mathrm{U}$ & $0.33 \mathrm{U}$ & $0.42 U$ & $0.26 \mathrm{U}$ & $0.32 U$ \\
\hline Colctum & 3530.00 & 3890.00 & 3780.00 & 6950.00 & 4190.00 & 4340.00 \\
\hline Chromiun & $2.10 \mathrm{~B}$ & $1.70 \mathrm{~B}$ & $1.50 \mathrm{U}$ & $2.30 \mathrm{~B}$ & $1.20 \mathrm{U}$ & $2.10 \mathrm{~B}$ \\
\hline Cobalt & $0.96 \mathrm{U}$ & $0.68 \mathrm{U}$ & $0.90 \mathrm{U}$ & $1.10 \mathrm{U}$ & $0.69 \mathrm{U}$ & 0.92 \\
\hline Copper & 5.808 & $5.70 \mathrm{~B}$ & $4.00 \mathrm{~B}$ & $7.60 \mathrm{~B}$ & $3.70 \mathrm{~B}$ & $6.30 \mathrm{~B}$ \\
\hline Iron & 258.00 & 436.00 & 109.00 & 355.00 & 593.00 & 1070.00 \\
\hline Lead & $3.80 \mathrm{~J}$ & $1.50 \mathrm{~J}$ & $0.72 \mathrm{~J}$ & $2.00 \mathrm{~J}$ & $4.20 \mathrm{~J}$ & $4.50 \mathrm{~J}$ \\
\hline Masnesicu & $1710.00 \mathrm{~B}$ & 2020.00 & 2310.00 & 3020.00 & 2620.00 & 2200.00 \\
\hline Manganese & 35.60 & 88.70 & 48.90 & 53.20 & 42.80 & 72.50 \\
\hline Mercury & $0.08 \mathrm{U}$ & $0.06 U$ & $0.08 \mathrm{U}$ & $0.09 \mathrm{U}$ & $0.06 U$ & $0.08 \mathrm{~B}$ \\
\hline Nickel & $1.90 \mathrm{U}$ & $1.40 \mathrm{U}$ & $1.80 \mathrm{U}$ & $2.30 \mathrm{U}$ & $1.90 \mathrm{~B}$ & $2.10 \mathrm{~B}$ \\
\hline Potuselun & 9110.00 & 9020.00 & 18000.00 & 21000.00 & 12900.00 & 10300.00 \\
\hline seleniun & $1.30 \mathrm{UJ}$ & $0.95 \mathrm{UJ}$ & 1.30 & 1.50 & $0.94 \mathrm{U}$ & $1.00 \mathrm{U}$ \\
\hline silver & $0.87 U$ & $0.62 u$ & $0.83 \mathrm{U}$ & $1.10 \mathrm{U}$ & $0.64 \mathrm{U}$ & $0.70 \mathrm{U}$ \\
\hline Sodiun & $98.80 \mathrm{~J}$ & $153.00 \downarrow$ & $144.00 \mathrm{~J}$ & $492.00 \mathrm{~J}$ & $75.20 \mathrm{~J}$ & $256.00 \mathrm{~J}$ \\
\hline Thallfum & 0.45 UJ & $0.32 \mathrm{UJ}$ & $0.43 \mathrm{UJ}$ & $0.51 \mathrm{UJ}$ & $0.32 \mathrm{UJ}$ & $0.36 \mathrm{UJ}$ \\
\hline Vanediun & $0.97 \mathrm{U}$ & $1.10 \mathrm{~B}$ & $0.93 \mathrm{U}$ & $1.20 \mathrm{U}$ & $1.30 \mathrm{~B}$ & $1.80 \mathrm{~B}$ \\
\hline Zine & $36.80 \mathrm{~J}$ & $65.40 \mathrm{~J}$ & $33.60 \mathrm{~J}$ & $69.80 \mathrm{~J}$ & $43.00 \mathrm{~J}$ & $56.10 \mathrm{~J}$ \\
\hline Cyanide & NR & NR & NR & NR & MR & NR \\
\hline C8-137 & $\mathbf{U}$ & U & $\mathbf{U}$ & $u$ & $\mathbf{u}$ & $\mathbf{u}$ \\
\hline $8 r=90$ & -0.20 & 0.20 & 0.06 & 2.40 & 2.90 & 0.02 \\
\hline
\end{tabular}

NOTE: All metals are reported in mg/kg. Radionuclides are reported in $\mathrm{pCi} / \mathrm{gm}$.

$B$ - Anulyte found in associated blank as well as semple.

$J$ - Not detected; associated value is estimated.

NR - Nonreportable.

$R$ = Dota are unusable.

$U$ = Not detected; value reported is semple quantitation limit.

UJ = Not detected; may not accurately reflect sample quantitation limit. 
Table C-10. Reed Canarygrass Sample Results from 100-H Reartur Area Collected Downriver and Upriver in 1992.

\begin{tabular}{|c|c|c|c|c|c|c|}
\hline \multirow{2}{*}{ Constituent } & \multicolumn{6}{|c|}{ Sample Identification Numbers } \\
\hline & 806469 & $806 N 70$ & $806 N 71$ & $806 N 72$ & B06N73 & BOSN74 \\
\hline Aluminum & $50.50 \mathrm{~B}$ & 110.00 & $38.80 \mathrm{~B}$ & $23.10 \mathrm{U}$ & 50.00 & 199.00 \\
\hline Ant imony & $5.50 \mathrm{U}$ & $3.90 \mathrm{U}$ & $4.20 \mathrm{U}$ & $4.00 \mathrm{U}$ & $4.70 \mathrm{U}$ & $3.90 \mathrm{U}$ \\
\hline Arsenle & $0.97 \mathrm{U}$ & $0.68 \mathrm{U}$ & $0.69 \mathrm{U}$ & $0.69 \mathrm{U}$ & $0.80 \mathrm{U}$ & $0.68 \mathrm{U}$ \\
\hline Bariun & 33.208 & $23.20 \mathrm{~B}$ & $17.90 \mathrm{~B}$ & $19.80 \mathrm{~B}$ & $22.60 \mathrm{~B}$ & $12.60 \mathrm{~B}$ \\
\hline Beryllium & $0.13 \mathrm{U}$ & $0.09 \mathrm{U}$ & $0.19 \mathrm{U}$ & $0.10 \mathrm{U}$ & $0.11 \mathrm{U}$ & $0.09 \mathrm{U}$ \\
\hline Cacminn & $0.32 u$ & $0.23 \mathrm{u}$ & $0.25 \mathrm{U}$ & $0.24 \mathrm{U}$ & $0.28 \mathrm{U}$ & $0.23 \mathrm{U}$ \\
\hline Calciun & 7390.00 & 6130.00 & 4340.00 & 5950.00 & 5380.00 & 1970.00 \\
\hline Chromiun & $1.50 \mathrm{U}$ & 2.30 & $1.20 \mathrm{~B}$ & 2.70 & $1.30 \mathrm{U}$ & 1.10 \\
\hline Cobalt & $0.87 \mathrm{U}$ & $0.62 \mathrm{U}$ & $0.67 \mathrm{U}$ & $0.64 U$ & $0.76 \mathrm{U}$ & $0.63 U$ \\
\hline Copper & 3.800 & $3.50 \mathrm{~B}$ & $3.00 \mathrm{~B}$ & $3.40 \mathrm{~B}$ & $5.40 \mathrm{~B}$ & $4.70 \mathrm{~B}$ \\
\hline Iron & 116.00 & 207.00 & 81.40 & 61.10 & 98.10 & 353.00 \\
\hline Leed & $0.67 \mathrm{UJ}$ & $0.89 \mathrm{~J}$ & $0.48 \mathrm{UJ}$ & $0.48 \mathrm{~J}$ & $1.70 \mathrm{~J}$ & $1.80 \mathrm{~J}$ \\
\hline Magnesiun & 3340.00 & 3660.00 & 2220.00 & 2650.00 & 2770.00 & 1190.00 \\
\hline Manganese & 45.30 & 24.80 & 39.40 & 11.30 & 11.10 & 37.30 \\
\hline Mercury & $0.08 \mathrm{U}$ & $0.06 \mathrm{U}$ & $0.06 \mathrm{U}$ & $0.06 \mathrm{U}$ & 0.14 & $0.10 \mathrm{~B}$ \\
\hline Nickel & $1.70 \mathrm{U}$ & $1.20 \mathrm{U}$ & $1.30 \mathrm{U}$ & $1.30 \mathrm{U}$ & $1.50 \mathrm{U}$ & $1.30 \mathrm{U}$ \\
\hline Potassiun & 12400.00 & 12100.00 & 15100.00 & 10700.00 & 14200.00 & 9900.00 \\
\hline seleniun & $1.30 \mathrm{U}$ & $2.30 \mathrm{U}$ & $0.91 \mathrm{U}$ & $0.90 \mathrm{U}$ & $1.00 \mathrm{U}$ & 0.89 UJ \\
\hline silver & $0.81 \cup$ & $0.57 \cup$ & $0.62 \mathrm{U}$ & $0.59 \mathrm{U}$ & $0.70 \mathrm{U}$ & $0.58 \cup$ \\
\hline Sodiun & $69.00 \mathrm{~J}$ & $98.10 \mathrm{~J}$ & $35.60 \mathrm{U}$ & $38.40 \mathrm{U}$ & $54.40 \mathrm{U}$ & $47.20 \mathrm{U}$ \\
\hline Thallium & 0.44 US & $0.30 \mathrm{UJ}$ & $0.31 \mathrm{uJ}$ & $0.31 \mathrm{UJ}$ & $0.36 \mathrm{UN}$ & $0.30 \mathrm{~J}$ \\
\hline Vanadium & $0.90 \mathrm{U}$ & $0.64 \mathrm{U}$ & $0.70 \mathrm{U}$ & $0.66 \mathrm{U}$ & $0.79 \mathrm{U}$ & 0.79 \\
\hline 2 Ine & $70.10 \mathrm{~J}$ & $64.70 \mathrm{~J}$ & $38.00 \mathrm{~J}$ & $74.80 \mathrm{~d}$ & $305.00 \mathrm{~J}$ & $95.80 \mathrm{~J}$ \\
\hline Cyanida & NR & NR & MR & NR & NR & NR \\
\hline C8.137 & $U$ & $U$ & $U$ & $u$ & U & U \\
\hline $8 r-90$ & 0.081 & 0.096 & 0.000 & 0.062 & -0.630 & 0.140 \\
\hline
\end{tabular}

NOTE: All motals are reported in mo/kg. Radionuclldes are reported in $\mathrm{pCl} / \mathrm{gm}$.

B - Analyte found in associated blank as well as sample.

$J$ - Not detected; aseociated value is estimated.

MR - Nonreportable.

$R$ - Date are unusable.

$U$ - Not detected; value reported is semple quantitation IImit.

UJ = Not detected; may not accurately reflect sample quantitation limit. 
Table C-11. Reed Canarygrass Sample Results from 100-D Reactor Area Collected Downriver and Upriver in 1992.

\begin{tabular}{|c|c|c|c|c|c|c|c|}
\hline \multirow{2}{*}{ Constituent } & \multicolumn{7}{|c|}{ Sample Identificat Ion Numbers } \\
\hline & BOSN7S & BO6N76 & BOSNT7 & BOSN78 & B06N79 & BO6N80 & $\begin{array}{l}\text { Control } \\
\text { BOSN81 }\end{array}$ \\
\hline Aluminum & $180.00 \mathrm{~J}$ & 118.00 & 60.80 & 273.00 & 273.00 & 535.00 & 207.00 \\
\hline Ant imony & $4.40 \mathrm{U}$ & $4.50 \mathrm{U}$ & $5.10 \mathrm{U}$ & $4.20 \mathrm{U}$ & $4.50 \mathrm{U}$ & $3.80 \mathrm{U}$ & $3.90 \mathrm{u}$ \\
\hline Arsenic & $0.98 \mathrm{~B}$ & $1.00 \mathrm{~B}$ & 0.85 UJ & $0.97 \mathrm{~B}$ & $0.73 \mathrm{U}$ & 0.66 UJ & $0.68 \mathrm{U}$ \\
\hline Barfun & $35.30 \mathrm{~B}$ & $14.30 \mathrm{~B}$ & $27.20 \mathrm{~B}$ & $21.70 \mathrm{~B}$ & $17.50 \mathrm{~B}$ & 21.908 & $14.80 \mathrm{~B}$ \\
\hline Beryllium & $0.10 \mathrm{U}$ & $0.11 \mathrm{u}$ & $0.12 \mathrm{U}$ & $0.39 \mathrm{U}$ & $0.11 \mathrm{U}$ & $0.46 \mathrm{U}$ & $0.09 \mathrm{U}$ \\
\hline Cectniun & $0.26 \mathrm{U}$ & $0.26 \mathrm{U}$ & $0.30 \mathrm{U}$ & $0.25 U$ & $0.27 \mathrm{U}$ & $0.23 \mathrm{U}$ & $0.23 \mathrm{U}$ \\
\hline calcium & 7030.00 & 2520.00 & 4060.00 & 4680.00 & 4430.00 & 5070.00 & 3560.00 \\
\hline Chromiun & 1.40 & $1.20 \mathrm{U}$ & $1.30 \mathrm{U}$ & 2.30 & $1.20 \mathrm{U}$ & $1.40 \mathrm{~B}$ & $1.60 \mathrm{~B}$ \\
\hline Cobalt & $0.70 \mathrm{U}$ & $0.71 \mathrm{U}$ & $0.81 \mathrm{U}$ & $0.70 \mathrm{~B}$ & $0.72 u$ & $0.62 \mathrm{U}$ & $0.63 \mathrm{U}$ \\
\hline Copper & $4.00 \mathrm{~B}$ & $3.30 \mathrm{~B}$ & $3.40 \mathrm{~B}$ & $4.90 \mathrm{~B}$ & $5.00 \mathrm{~B}$ & 5.10 & $4.40 \mathrm{~B}$ \\
\hline Iron & 382.00 & 231.00 & 128.00 & 491.00 & 514.00 & 907.00 & 426.00 \\
\hline Lead & $2.30 \mathrm{~J}$ & $1.00 \mathrm{~J}$ & $0.68 \mathrm{~J}$ & $2.70 \mathrm{~J}$ & $0.91 \mathrm{~J}$ & $3.80 \mathrm{~J}$ & $1.20 \mathrm{~J}$ \\
\hline Magnes ivn & 2730.00 & 1680.00 & 2080.00 & 2050.00 & 1830.00 & 1800.00 & 1890.00 \\
\hline Manganese & 58.50 & 31.20 & 39.90 & 84.40 & 64.80 & 55.10 & 58.10 \\
\hline Marcury & $0.06 U$ & $0.05 \mathrm{U}$ & $0.07 \mathrm{U}$ & $0.05 \mathrm{U}$ & $0.06 \mathrm{U}$ & $0.05 \cup$ & $0.06 \mathrm{U}$ \\
\hline Nlckal & $1.40 \mathrm{U}$ & $1.40 \mathrm{U}$ & $1.60 \mathrm{U}$ & $2.30 \mathrm{~B}$ & $1.40 \mathrm{U}$ & $1.60 \mathrm{~B}$ & $1.30 \mathrm{U}$ \\
\hline Potases/un & 12700.00 & 12400.00 & 10100.00 & 9190.00 & 11200.00 & 7780.00 & 11800.00 \\
\hline seleniun & $0.99 \mathrm{U}$ & $0.99 \cup$ & $1.10 \mathrm{U}$ & $0.95 \mathrm{U}$ & $0.98 \mathrm{UJ}$ & $0.87 \mathrm{U}$ & $0.89 \mathrm{U}$ \\
\hline silver & $0.65 \mathrm{U}$ & $0.66 \mathrm{U}$ & $0.73 \mathrm{U}$ & $0.62 \mathrm{U}$ & $0.67 \mathrm{U}$ & $0.57 \mathrm{U}$ & $0.58 \mathrm{U}$ \\
\hline sodium & $94.60 \mathrm{~J}$ & $59.20 \mathrm{~J}$ & $147.00 \mathrm{~J}$ & $103.00 \mathrm{~J}$ & $150.00 \mathrm{~J}$ & $218.00 \mathrm{~J}$ & $139.00 \mathrm{~J}$ \\
\hline Thallitum & $0.34 \mathrm{UJ}$ & $0.34 U_{J}$ & $0.38 \mathrm{UJ}$ & $0.32 u$ & 0.33 Ud & $0.30 \mathrm{U}$ & $0.3 \mathrm{U} \mathrm{UJ}$ \\
\hline Vanediun & $0.7 \mathrm{U}$ & $0.74 \mathrm{U}$ & $0.84 \mathrm{U}$ & $1.00 \mathrm{~B}$ & $0.75 \mathrm{U}$ & $1.50 \mathrm{~B}$ & 0.81 \\
\hline ZIne & $70.50 \mathrm{~J}$ & $59.40 \mathrm{~J}$ & $143.00 \mathrm{~J}$ & $91.10 \mathrm{~J}$ & $47.30 \mathrm{~J}$ & $51.80 \mathrm{~J}$ & $69.60 \mathrm{~J}$ \\
\hline Cyanida & MR & NR & NR & NR & MR & NR & NR \\
\hline Cs-137 & $u$ & $\mathbf{u}$ & $u$ & $U$ & u & $U$ & $u$ \\
\hline $8 r-90$ & .069 & 0.041 & 0.06 & $\cdot 0.28$ & -0.17 & -0.36 & 0.009 \\
\hline
\end{tabular}

MOTE: All metals are reported in ma/kg. Radionuclldes are reported in pci/g.

B Analyte found In assoclated blank os well as sample.

$J$ - Not detected associated value is estimated.

NR = Nonreportable.

R - Date are unuenble.

$U$ - Not detected; value reported is semple quantitation IImit.

UJ a Not detected; may not eccurately reflect semple quantitation I imlt. 
Table C-12. Reed Canarygrass Sample Results from 100-K Reactor Area Collected Downriver and Upriver in 1992.

\begin{tabular}{|c|c|c|c|c|c|c|}
\hline \multirow{2}{*}{ Const Itwent } & \multicolumn{6}{|c|}{ sample Identiffection Numbers } \\
\hline & sosuse & coswaz & BOSNE4 & 806N85 & 8061486 & BOSNA7 \\
\hline Aluninum & 97.30 & 238.00 & 116.00 & 399.00 & 125.00 & 78.80 \\
\hline Ant Inomy & $4.00 \mathrm{U}$ & $4.20 \mathrm{~V}$ & $4.30 \mathrm{U}$ & $4.30 \mathrm{U}$ & $3.30 \mathrm{~V}$ & $4.00 \mathrm{~V}$ \\
\hline Arsenic & $0.77 \mathrm{~J}$ & $1.10 \mathrm{~J}$ & $0.98 \mathrm{~J}$ & $1.30 \mathrm{~J}$ & $1.10 \mathrm{~J}$ & $0.90 \mathrm{~J}$ \\
\hline Barfun & 12.808 & 23.20 & $14.20 \mathrm{~B}$ & $16.90 \mathrm{~B}$ & $16.60 \mathrm{~B}$ & 20.20 \\
\hline Derylliun & $0.10 \mathrm{U}$ & $0.10 \mathrm{U}$ & $0.10 \mathrm{U}$ & $0.10 \mathrm{U}$ & $0.08 \mathrm{U}$ & $0.09 \mathrm{U}$ \\
\hline coctaiun & $0.26 \mathrm{U}$ & $0.37 \mathrm{U}$ & $0.38 \mathrm{U}$ & $0.38 \mathrm{U}$ & $0.38 \mathrm{U}$ & 1.50 \\
\hline Calctum & 2920.00 & $3930.00 \mathrm{~J}$ & $2860.00 \mathrm{~J}$ & $3170.00 \mathrm{~J}$ & $3730.00 \mathrm{~J}$ & $2890.00 \mathrm{~J}$ \\
\hline Chromicn & $1.10 \mathrm{U}$ & $1.10 \mathrm{U}$ & $1.10 \mathrm{~V}$ & $1.10 \mathrm{U}$ & $0.89 \mathrm{U}$ & $1.10 \mathrm{~V}$ \\
\hline Cobalt & $0.4 \mathrm{U}$ & $0.67 \mathrm{U}$ & $0.69 \mathrm{U}$ & $0.69 \mathrm{U}$ & $0.53 U$ & $0.64 \mathrm{~V}$ \\
\hline Copper & $4.60 \mathrm{~B}$ & 6.50 & 6.50 & 7.80 & 8.60 & $4.10 \mathrm{~V}$ \\
\hline Iron & 199.00 & $511.00 \mathrm{~N}$ & $250.00 \mathrm{~J}$ & $720.00 \mathrm{~J}$ & $260.00 \mathrm{~J}$ & $139.00 \mathrm{~J}$ \\
\hline Lead & $1.10 \mathrm{~J}$ & 3.40 & 1.40 & 4.30 & 3.20 & 1.00 \\
\hline Magnestun & 1990.00 & 2100.00 & 1580.00 & 1910.00 & 2470.00 & 2390.00 \\
\hline Mangarnese & 77.40 & $96.00 \mathrm{~J}$ & 57.60 & $47.90 \mathrm{~J}$ & $45.90 \mathrm{~J}$ & $25.70 \mathrm{~J}$ \\
\hline Mercury & $0.06 \mathrm{~V}$ & 0.05 UJ & $0.10 \mathrm{~J}$ & $0.06 \mathrm{~J}$ & 0.05 Ud & $0.05 \mathrm{us}$ \\
\hline Nickol & $1.30 \mathrm{U}$ & $1.30 \mathrm{~V}$ & $1.40 \mathrm{~V}$ & 4.200 & 1.50 & $1.30 \mathrm{U}$ \\
\hline Poresestun & 16400.00 & $12400.00 \mathrm{~J}$ & $12100.00 \mathrm{~J}$ & $9920.00 \mathrm{~J}$ & $12700.00 \mathrm{~J}$ & $14300,00 \mathrm{~J}$ \\
\hline soleniun & $0.90 \mathrm{U}$ & $0.96 \mathrm{UJ}$ & 0.90 UJ & $1.10 \mathrm{~B}$ & 0.73 UJ & $1.10 \mathrm{~B}$ \\
\hline sllver & $0.59 \mathrm{U}$ & $0.89 \mathrm{U}$ & $0.92 \mathrm{U}$ & $0.92 \mathrm{U}$ & $0.71 \mathrm{U}$ & $0.85 \mathrm{U}$ \\
\hline sodiun & $129.00 \mathrm{~J}$ & $91.90 \mathrm{U}$ & $38.70 \mathrm{U}$ & $85.30 \mathrm{U}$ & $84.40 \mathrm{U}$ & $33.80 \mathrm{U}$ \\
\hline Thallium & $0.31 \mathrm{U}$ & $0.42 \mathrm{U}$ & $0.43 \mathrm{U}$ & 0.74 & $0.33 \mathrm{u}$ & $0.40 \mathrm{Ud}$ \\
\hline Venadiun & $0.66 \mathrm{U}$ & $0.69 \mathrm{~V}$ & $0.71 \mathrm{U}$ & $0.71 \mathrm{U}$ & $0.55 \mathrm{U}$ & $0.66 \mathrm{~V}$ \\
\hline $2 \ln e$ & $97.40 \mathrm{~J}$ & $75.30 \mathrm{~J}$ & $147.00 \mathrm{~d}$ & $192.00 \mathrm{~J}$ & $112.00 \mathrm{~J}$ & $128.00 \mathrm{~J}$ \\
\hline Crenide & NR & NR & NR & Hn & MR & MR \\
\hline 68.137 & U & $u$ & 0.23 & $u$ & $u$ & $u$ \\
\hline $5 r-90$ & .0 .850 & $0.430 \mathrm{R}$ & $0.011 \mathrm{Ud}$ & 0.029 UJ & $0.00 \mathrm{UJ}$ & 0.14 US \\
\hline
\end{tabular}

Notes All matals ore reported in $\mathrm{mg} / \mathrm{kg}$. Radionuclidas are reported in $\mathrm{pCl} / \mathrm{om}$.

B - Analyte found in assoclated blank as well us semple.

$\mathrm{J}$ - Not dotectudi aseociated value is estimated.

MR - Nonreportable.

R. Dato are unubeble.

$U$ - Not detecteds value reported is emple quantitution IImit.

UJ - Not datected; may not eccurately reflect semple quantitation IImit. 
Table C-13. Reed Canarygrass Sample Results from 100-BC Reactor Area Collected Downriver and Upriver in 1992.

\begin{tabular}{|c|c|c|c|c|c|c|c|}
\hline \multirow{2}{*}{ Const I tuent } & \multicolumn{7}{|c|}{ semple Identiflcation Numbers } \\
\hline & cosnes & 806N89 & BOSN90 & Bo6N91 & BO6N92 & BOSN93 & $\begin{array}{l}\text { Control } \\
\text { sosNO4 }\end{array}$ \\
\hline Aluainum & 120.00 & 99.60 & 78.30 & 190.00 & 113.00 & 132.00 & 101.00 \\
\hline Ant Inomy & $3.90 \mathrm{U}$ & $3.90 \mathrm{U}$ & $6.00 \mathrm{U}$ & $4.90 \mathrm{U}$ & $4.20 \mathrm{~V}$ & $5.50 \mathrm{U}$ & $4.50 \mathrm{U}$ \\
\hline Arsente & $0.77 \mathrm{~J}$ & 0.73 U & $0.76 \mathrm{US}$ & $1.70 \mathrm{~J}$ & $1.30 \mathrm{~J}$ & $1.30 \mathrm{~J}$ & $1.20 \mathrm{~J}$ \\
\hline Derlum & $16.40 \mathrm{D}$ & $18.00 \mathrm{C}$ & 15.908 & $18.40 \mathrm{~B}$ & 11.600 & 12.70 & 10.10 \\
\hline Beryll lun & $0.09 \mathrm{U}$ & $0.09 \mathrm{~V}$ & $0.10 \mathrm{U}$ & $0.12 \mathrm{U}$ & $0.10 \mathrm{U}$ & $0.13 \mathrm{U}$ & $0.11 \mathrm{v}$ \\
\hline Ceconilum & $0.35 \mathrm{U}$ & $0.35 \mathrm{U}$ & $0.36 \mathrm{U}$ & $0.44 \mathrm{U}$ & $0.37 \mathrm{U}$ & $0.49 \mathrm{U}$ & $0.40 \mathrm{~V}$ \\
\hline Colclum & $5110.00 \mathrm{~J}$ & $6730.00 \mathrm{~J}$ & $5870.00 \mathrm{~J}$ & $5950.00 \mathrm{~J}$ & $3000.00 \mathrm{~J}$ & $3310.00 \mathrm{~J}$ & $2360.00 \mathrm{~J}$ \\
\hline Chroulun & $1.00 \mathrm{~V}$ & $1.00 \mathrm{U}$ & $1.10 \mathrm{U}$ & $1.30 \mathrm{U}$ & $1.10 \mathrm{U}$ & $1.30 \mathrm{U}$ & $1.20 \mathrm{U}$ \\
\hline Cobolt & $0.62 \mathrm{v}$ & $0.62 \mathrm{U}$ & $0.64 v$ & $0.79 \mathrm{U}$ & $0.67 \mathrm{U}$ & $0.88 \mathrm{U}$ & $0.72 \mathrm{U}$ \\
\hline Copper & $2.70 \mathrm{u}$ & $3.30 \mathrm{U}$ & $2.80 \mathrm{U}$ & 2.20 & $3.00 \mathrm{U}$ & $2.50 \mathrm{U}$ & $2.90 \mathrm{U}$ \\
\hline Iron & $232.00 \mathrm{~J}$ & $208.00 \mathrm{~J}$ & $167.00 \mathrm{~J}$ & $350.00 \mathrm{~J}$ & $227.00 \mathrm{~J}$ & $249.00 \mathrm{~J}$ & $194.00 \mathrm{~J}$ \\
\hline Lead & 1.60 & $1.80 \mathrm{~J}$ & $0.83 \mathrm{~J}$ & $1.80 \mathrm{~J}$ & 1.60 & 1.70 & 1.60 \\
\hline Magnestun & 2670.00 & 2020.00 & 3020.00 & 2350.00 & 2580.00 & 2130.00 & 1180.00 \\
\hline Mengeneses & $\mathbf{6 3} .30 \mathrm{~J}$ & $47.10 \mathrm{~J}$ & $77.10 \mathrm{~J}$ & $57.20 \mathrm{~J}$ & $74.70 \mathrm{~J}$ & $63.40 \mathrm{~J}$ & $22.00 \mathrm{~J}$ \\
\hline Meroury & Mn & 0.03 Ud & $0.06 \mathrm{uJ}$ & 0.07 UJ & 0.05 UJ & $0.08 \mathrm{UJ}$ & $0.07 \mathrm{UJ}$ \\
\hline Nickol & $1.20 \mathrm{U}$ & $1.20 \mathrm{U}$ & $1.30 \mathrm{U}$ & $1.60 \mathrm{~V}$ & $1.30 \mathrm{U}$ & $1.80 \mathrm{U}$ & $1.40 \mathrm{U}$ \\
\hline Potaselum & $10600.00 \mathrm{~J}$ & $9170.00 \mathrm{~J}$ & $11300.00 \mathrm{~J}$ & $15530.00 \mathrm{~J}$ & $9430,00 \mathrm{~J}$ & $7460.00 \mathrm{~J}$ & $7390.00 \mathrm{~J}$ \\
\hline soteniven & $0.94 \mathrm{u}$ & $0.92 \mathrm{U}$ & $0.92 \mathrm{U}$ & $1.10 \mathrm{U}$ & 1.30 & $1.30 \mathrm{UJ}$ & $1.10 \mathrm{UJ}$ \\
\hline 81 liver & $0.83 u$ & $0.83 \mathrm{U}$ & $0.85 \mathrm{U}$ & $1.10 \mathrm{U}$ & $0.90 \mathrm{U}$ & $1.20 \mathrm{~V}$ & $0.96 \mathrm{U}$ \\
\hline sedium & $44.70 \mathrm{~V}$ & $44.80 \mathrm{U}$ & $44.30 \mathrm{U}$ & $257.00 \mathrm{~J}$ & $193.00 \mathrm{~J}$ & $152.00 \mathrm{~J}$ & $48.90 \mathrm{U}$ \\
\hline Thalllum & $0.41 \mathrm{U}$ & 0.668 & $0.40 \mathrm{U}$ & 0.49 UJ & 0.43 UJ & $0.56 \mathrm{U}$ & $0.47 \mathrm{U}$ \\
\hline Vanediven & $0.65 \mathrm{U}$ & $0.65 \mathrm{u}$ & $0.66 \mathrm{U}$ & $0.82 \mathrm{U}$ & $0.70 \mathrm{~V}$ & $0.92 \mathrm{U}$ & $0.7 \mathrm{u}$ \\
\hline Zine & $68.10 \mathrm{~J}$ & $66.80 \mathrm{~J}$ & $41.60 \mathrm{~J}$ & $98.10 \mathrm{~J}$ & $121.00 \mathrm{~J}$ & $115.00 \mathrm{~J}$ & $49.60 \mathrm{~J}$ \\
\hline Crentide & NR & Mn & NR & MR & NR & NR & MR \\
\hline Ca.137 & u & $u$ & $u$ & $u$ & $u$ & $u$ & $\mathbf{u}$ \\
\hline $8 r \cdot 90$ & .0 .10 Ud & .0 .34 Ud & 0.27 Us & $0.16 \mathrm{UJ}$ & $0.20 \mathrm{UJ}$ & 0.024 UN & $.0 .29 \mathrm{UJ}$ \\
\hline
\end{tabular}

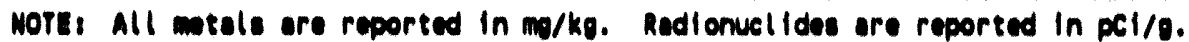

- Analyte found in assoclated blank as woll as sample.

$J$ - Not dotected asseciated value lo estimated.

Mn - Nenreportable.

R a Dota are uniseble.

$U$ - Mot datected valus reported is scmple quantitution $1 /$ mit.

UJ - Not detectedi my not aceurately refloct semple quantitation IImit. 
WHC-EP-0620

This page intentionally left blank. 
WHC-EP. 0620

\section{APPENDIX D}

ANALYTICAL RESULTS FOR TREES 
WHC-EP-0620

This page intentionally left blank. 
Table D-1. Tree Leaf Sample Results Collected from 100 Areas in 1991. (sheet 1 of 2)

\begin{tabular}{|c|c|c|c|c|c|c|c|c|}
\hline \multirow{3}{*}{ Conse I tuent } & \multicolumn{8}{|c|}{ Sumple Identilficetion Numbers and Location } \\
\hline & Control & Control & Belon 0 & Below D & Belon 0 & Belon D & Bolow 0 & Below 0 \\
\hline & soovis & scowo & $\begin{array}{l}\text { coovis" } \\
\text { (1101053) }\end{array}$ & $\begin{array}{l}\text { soovis" } \\
(1001094)\end{array}$ & $\begin{array}{l}\text { colos3 } \\
\text { Dupllicate }\end{array}$ & $\begin{array}{l}\text { co10sh } \\
\text { Dupl leate }\end{array}$ & $\cos \times 3$ & $800 \mathrm{~V} \times 4$ \\
\hline Aluminn & $2700.00 \mathrm{~J}$ & $352.00 \mathrm{~J}$ & $746.00 \mathrm{~J}$ & $281.00 \mathrm{~J}$ & $354.00 \mathrm{~J}$ & $301.00 \mathrm{~J}$ & $433.00 \mathrm{~J}$ & $499.00 \mathrm{~J}$ \\
\hline Ant Imony & $3.20 \mathrm{UJ}$ & $3.50 \mathrm{~J}$ & $3.60 \mathrm{~J}$ & $3.20 \mathrm{uJ}$ & $4.90 \mathrm{~J}$ & $3.20 \mathrm{uJ}$ & $3.20 \mathrm{UJ}$ & $3.20 \mathrm{UJ}$ \\
\hline Arsente & $1.00 \mathrm{UJ}$ & 1.00 Ud & $1.00 \mathrm{UJ}$ & $1.00 \mathrm{UJ}$ & $1.00 \mathrm{UJ}$ & $1.00 \mathrm{UJ}$ & $1.00 \mathrm{UJ}$ & $1.00 \mathrm{uJ}$ \\
\hline Barluan & $216.00 \mathrm{~J}$ & $125.00 \mathrm{~J}$ & $136.00 \mathrm{~J}$ & $168.00 \mathrm{~J}$ & $151.00 \mathrm{~J}$ & $117.00 \mathrm{~J}$ & $199.00 \mathrm{~J}$ & $129.00 \mathrm{~J}$ \\
\hline Derylliun & $0.20 \mathrm{UJ}$ & $0.20 \mathrm{uJ}$ & $0.20 \mathrm{UJ}$ & $0.20 \mathrm{UJ}$ & $0.20 \mathrm{UJ}$ & $0.20 \mathrm{UJ}$ & $0.20 \mathrm{uJ}$ & $0.20 \mathrm{UJ}$ \\
\hline Cadilun & $1.20 \mathrm{~J}$ & $0.40 \mathrm{UJ}$ & $0.60 \mathrm{UJ}$ & $0.62 \mathrm{~J}$ & $0.60 \mathrm{uJ}$ & $0.60 \mathrm{UJ}$ & $0.40 \mathrm{UJ}$ & $0.60 \mathrm{uJ}$ \\
\hline Calefin & $113000.00 \mathrm{~d}$ & $121000.00 \mathrm{~J}$ & $113000.00 \mathrm{~J}$ & $97800.00 \mathrm{~J}$ & $119000.00 \mathrm{~J}$ & $118000.00 \mathrm{~J}$ & $121000.00 \mathrm{~J}$ & $121000.00 \mathrm{~J}$ \\
\hline Chromilum & $6.80 \mathrm{~J}$ & $2.10 \mathrm{~J}$ & $1.30 \mathrm{~J}$ & $1.70 \mathrm{~J}$ & $2.00 \mathrm{~J}$ & $1.80 \mathrm{~J}$ & $4.30 \mathrm{~J}$ & $2.80 \mathrm{~J}$ \\
\hline Cobalt & $2.00 \mathrm{~J}$ & $0.90 \mathrm{~J}$ & $0.60 \mathrm{UN}$ & $1.20 \mathrm{~J}$ & $0.90 \mathrm{~J}$ & $1.10 \mathrm{~J}$ & $0.64 \mathrm{~J}$ & $1.10 \mathrm{~J}$ \\
\hline Coppor & $39.80 \mathrm{~J}$ & $19.70 \mathrm{~J}$ & $23.40 \mathrm{~J}$ & $34.00 \mathrm{~J}$ & $24.70 \mathrm{~J}$ & $34.40 \mathrm{~J}$ & $27.40 \mathrm{~J}$ & $24.80 \mathrm{~J}$ \\
\hline Iron & $3990.00 \mathrm{~J}$ & $600.00 \mathrm{~J}$ & $67.00 \mathrm{~J}$ & $604.00 \mathrm{~J}$ & $660.00 \mathrm{~J}$ & $729.00 \mathrm{~J}$ & $790.00 \mathrm{~J}$ & $864.00 \mathrm{~J}$ \\
\hline Leed & $7.10 \mathrm{~J}$ & $1.00 \mathrm{~J}$ & $0.91 \mathrm{~J}$ & $1.10 \mathrm{~J}$ & $1.00 \mathrm{~J}$ & $1.10 \mathrm{~J}$ & $0.98 \mathrm{~J}$ & $1.10 \mathrm{~J}$ \\
\hline Mognee Iun & $27900.00 \mathrm{~J}$ & $26400.00 \mathrm{~J}$ & $16600.00 \mathrm{~J}$ & $12300.00 \mathrm{~J}$ & $12800.00 \mathrm{~J}$ & $23500.00 \mathrm{~J}$ & $22500.00 \mathrm{~J}$ & $25500.00 \mathrm{~J}$ \\
\hline Mengenese & $176.00 \mathrm{~J}$ & $61.30 \mathrm{~J}$ & $199.00 \mathrm{~J}$ & $125.00 \mathrm{~J}$ & $125.00 \mathrm{~J}$ & $200.00 \mathrm{~J}$ & $98.00 \mathrm{~J}$ & $85.10 \mathrm{~J}$ \\
\hline Nickol & $5.30 \mathrm{~J}$ & $1.00 \mathrm{~J}$ & $4.40 \mathrm{~J}$ & $6.40 \mathrm{~J}$ & $4.60 \mathrm{~J}$ & $6.60 \mathrm{~J}$ & $1.00 \mathrm{~J}$ & $1.40 \mathrm{~J}$ \\
\hline Potessivn & $6 \$ 500.00 \mathrm{~J}$ & $41400.00 \mathrm{~J}$ & $87600.00 \mathrm{~J}$ & $113000.00 \mathrm{~d}$ & $89600.00 \mathrm{~J}$ & $97000.00 \mathrm{~J}$ & $93600.00 \mathrm{~J}$ & $73400.00 \mathrm{~J}$ \\
\hline seleniun & $6.00 \mathrm{Ud}$ & $6.00 \mathrm{uJ}$ & $4.00 \mathrm{UJ}$ & $4.00 \mathrm{UJ}$ & $6.00 \mathrm{UJ}$ & $4.00 \mathrm{UJ}$ & $4.00 \mathrm{UJ}$ & $4.00 \mathrm{UJ}$ \\
\hline sllver & $1.20 \mathrm{~J}$ & $1.30 \mathrm{~J}$ & $0.40 \mathrm{UJ}$ & $0.40 \mathrm{UJ}$ & 0.40 Ud & $0.98 \mathrm{~J}$ & $0.76 \mathrm{~J}$ & $0.92 \mathrm{~J}$ \\
\hline sodiun & $1070.00 \mathrm{~J}$ & $573.00 \mathrm{~J}$ & $361.00 \mathrm{~J}$ & $362.00 \mathrm{~J}$ & $450.00 \mathrm{~J}$ & $316.00 \mathrm{~J}$ & $1020.00 \mathrm{~J}$ & $1610.00 \mathrm{~J}$ \\
\hline inalliven & $0.40 \mathrm{UJ}$ & $0.40 \mathrm{Ud}$ & $0.40 \mathrm{UJ}$ & $0.40 \mathrm{UJ}$ & 0.40 UN & $0.40 \mathrm{uJ}$ & $0.40 \mathrm{uJ}$ & $0.40 \mathrm{UJ}$ \\
\hline Vanediun & $6.80 \mathrm{~J}$ & $0.92 \mathrm{~J}$ & $0.75 \mathrm{~J}$ & $1.00 \mathrm{~J}$ & $1.20 \mathrm{~J}$ & $0.96 \mathrm{~J}$ & $0.60 \mathrm{~J}$ & $1.20 \mathrm{~J}$ \\
\hline $21 n$ & $261.00 \mathrm{~J}$ & $118.00 \mathrm{~J}$ & $142.00 \mathrm{~J}$ & $195.00 \mathrm{~J}$ & $143.00 \mathrm{~J}$ & $160.00 \mathrm{~J}$ & $220.00 \mathrm{~J}$ & $247.00 \mathrm{~J}$ \\
\hline Cranlde & Mn & Mn & MR & NR & MR & MR & NR & NR \\
\hline $68 \cdot 137$ & -.2072 us & 0.1545 UJ & $\$ 1.161 \mathrm{uJ}$ & 0.9873 UJ & $<.6489 \mathrm{UJ}$ & $<.5676$ UN & $\$ .6530 \mathrm{UJ}$ & $<.6125 \mathrm{UJ}$ \\
\hline $8 r .90$ & $.07 \mathrm{n}$ & $0.18 \mathrm{UJ}$ & $0.29 R$ & $0.03 R$ & $=0.10 \mathrm{UJ}$ & $0.10 \mathrm{UJ}$ & $.2 .00 \mathrm{uJ}$ & $0.17 \mathrm{UJ}$ \\
\hline 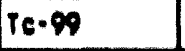 & .1 UJ & $0.05 \mathrm{US}$ & $0.10 \mathrm{UJ}$ & $0.20 \mathrm{UJ}$ & $0.50 \mathrm{R}$ & $0.60 \mathrm{~J}$ & $0.20 R$ & $0.40 \mathrm{~J}$ \\
\hline
\end{tabular}

Motel All metels are reported in ma/kg. Redlonuclldes are reported in pCl/o.

- Anolyte found In assocleted blenk as woll as ample.

$J$ - Not detectedi assoclated value ls estimated.

NR - Nonreportablo.

- Dato ore unuabible.

$U$ - Not dateetedi value reported le semple quantitation IImit.

UN - Not dateeted; may nor accurutely roflect semple quantitation IImit.

bupll leote semple taken. 
Table D-1. Tree Leaf Sample Results Collected from 100 Areas in 1991. (sheet 2 of 2 )

\begin{tabular}{|c|c|c|c|c|c|c|c|c|}
\hline \multirow{3}{*}{ Const Ituent } & \multicolumn{8}{|c|}{ Sample Identification Numbers and Locutions } \\
\hline & Below N & Below $N$ & Below I & Below F & Belon BC & Below BC & Above $H$ & Above 11 \\
\hline & coovrs & LoOV20 & s00V27 & Boov28 & Boovi & noovv8 & Boovr1 & Boovra \\
\hline Alvinim & $291.00 \mathrm{~J}$ & $1500.00 \mathrm{~J}$ & $226.00 \mathrm{~J}$ & $301.00 \mathrm{~J}$ & $317.00 \mathrm{~J}$ & $460.00 \mathrm{~J}$ & $1030.00 \mathrm{~J}$ & $561.00 \mathrm{~J}$ \\
\hline Ant Imeny & $3.20 \mathrm{UJ}$ & $3.20 \mathrm{Ud}$ & $3.40 \mathrm{~J}$ & $3.20 \mathrm{~J}$ & $3.20 \mathrm{UJ}$ & $3.20 \mathrm{UJ}$ & $3.20 \mathrm{UJ}$ & $3.20 \mathrm{UJ}$ \\
\hline Arsenic & $1.00 \mathrm{UJ}$ & $1.00 \mathrm{uJ}$ & 1.00 vo & $1.00 \mathrm{uJ}$ & $1.60 \mathrm{~J}$ & 1.00 UJ & $1.00 \mathrm{Ud}$ & $1.00 \mathrm{UJ}$ \\
\hline Doriun & $199.00 \mathrm{~J}$ & $222.00 \mathrm{~J}$ & $124.00 \mathrm{~J}$ & $235.00 \mathrm{~J}$ & $216.00 \mathrm{~J}$ & $252.00 \mathrm{~J}$ & $306.00 \mathrm{~J}$ & $189.00 \mathrm{~J}$ \\
\hline Beryllium & $0.20 \mathrm{UJ}$ & $0.20 \mathrm{UJ}$ & $0.20 \mathrm{UJ}$ & $0.20 \mathrm{UJ}$ & $0.20 \mathrm{uJ}$ & $0.20 \mathrm{UJ}$ & $0.20 \mathrm{UJ}$ & $0.20 \mathrm{UJ}$ \\
\hline Cectaicu & $0.40 \mathrm{UJ}$ & 0.40 UJ & $1.50 \mathrm{~J}$ & $0.44 \mathrm{~J}$ & $0.40 \mathrm{UJ}$ & $0.48 \mathrm{~J}$ & $0.98 \mathrm{~J}$ & $0.78 \mathrm{~J}$ \\
\hline calciun & 164000.00 & $142000.00 \mathrm{~J}$ & $123000.00 \mathrm{~J}$ & $140000.00 \mathrm{~J}$ & $118000.00 \mathrm{~J}$ & $132000.00 \mathrm{~J}$ & $134000.00 \mathrm{~J}$ & $165000.00 \mathrm{~J}$ \\
\hline Chromiun & $1.80 \mathrm{~J}$ & $2.30 \mathrm{~J}$ & $1.90 \mathrm{~J}$ & $3.20 \mathrm{~J}$ & $1.30 \mathrm{~J}$ & $2.20 \mathrm{~J}$ & $3.30 \mathrm{~J}$ & $3.70 \mathrm{~J}$ \\
\hline Cobalt & $0.45 \mathrm{~J}$ & $0.4 \mathrm{~J}$ & $4.40 \mathrm{~J}$ & 0.96 & $0.60 \mathrm{uJ}$ & $1.20 \mathrm{~J}$ & $1.10 \mathrm{~J}$ & $0.85 \mathrm{~J}$ \\
\hline coppor & $34.30 \mathrm{~J}$ & $39.30 \mathrm{~J}$ & $36.20 \mathrm{~J}$ & $39.40 \mathrm{~J}$ & $27.50 \mathrm{~J}$ & $20.80 \mathrm{~J}$ & $92.50 \mathrm{~J}$ & $60.80 \mathrm{~J}$ \\
\hline iron & $900.00 \mathrm{~J}$ & $817.00 \mathrm{~J}$ & $627.00 \mathrm{~J}$ & $722.00 \mathrm{~J}$ & $699.00 \mathrm{~J}$ & $839.00 \mathrm{~J}$ & $1170.00 \mathrm{~J}$ & $1410.00 \mathrm{~J}$ \\
\hline Leed & $1.60 \mathrm{~J}$ & $1.30 \mathrm{~J}$ & $0.67 \mathrm{~J}$ & $1.30 \mathrm{~J}$ & $1.30 \mathrm{~J}$ & $2.30 \mathrm{~J}$ & $3.20 \mathrm{~J}$ & $5.60 \mathrm{~J}$ \\
\hline Meonosive & $19300.00 \mathrm{~J}$ & $20100.00 \mathrm{~J}$ & $46000.00 \mathrm{~J}$ & $19200.00 \mathrm{~J}$ & $17100.00 \mathrm{~J}$ & $13000.00 \mathrm{~J}$ & $21700.00 \mathrm{~J}$ & $37500.00 \mathrm{~J}$ \\
\hline Manganese & $258.00 \mathrm{~J}$ & $125.00 \mathrm{~J}$ & $288.00 \mathrm{~J}$ & $187.00 \mathrm{~J}$ & $86.70 \mathrm{~J}$ & $139.00 \mathrm{~J}$ & $265.00 \mathrm{~J}$ & $377.00 \mathrm{~J}$ \\
\hline Mickel & $1.80 \mathrm{~J}$ & $2.20 \mathrm{~J}$ & $1.50 \mathrm{~J}$ & $3.50 \mathrm{~J}$ & $2.90 \mathrm{~J}$ & $3.60 \mathrm{~J}$ & $6.50 \mathrm{~J}$ & $7.40 \mathrm{~J}$ \\
\hline Potaselin & $114000.00 \mathrm{~J}$ & $96000.00 \mathrm{~J}$ & $98500.00 \mathrm{~J}$ & $112000.00 \mathrm{~J}$ & $112000.00 \mathrm{~J}$ & $72100.00 \mathrm{~J}$ & $200000.00 \mathrm{~J}$ & $199000.00 \mathrm{~J}$ \\
\hline seleniun & $4.00 \mathrm{UN}$ & $4.00 \mathrm{UN}$ & $4.00 \mathrm{UJ}$ & $4.00 \mathrm{UJ}$ & $5.50 \mathrm{~J}$ & $4.00 \mathrm{UN}$ & $4.00 \mathrm{n}$ & $4.00 R$ \\
\hline sllver & $0.71 \mathrm{~J}$ & $0.76 \mathrm{~d}$ & $2.80 \mathrm{~J}$ & $0.30 \mathrm{~J}$ & $0.40 \mathrm{uJ}$ & $0.40 \mathrm{~J}$ & $0.52 \mathrm{~J}$ & $1.90 \mathrm{~J}$ \\
\hline sodiun & $260.00 \mathrm{~J}$ & $308.00 \mathrm{~J}$ & $961.00 \mathrm{~J}$ & $195.00 \mathrm{~J}$ & $369.00 \mathrm{~J}$ & $628.00 \mathrm{~J}$ & $614.00 \mathrm{~J}$ & $660.00 \mathrm{~J}$ \\
\hline Thalliven & $0.40 \mathrm{UN}$ & $0.60 \mathrm{UJ}$ & $0.60 \mathrm{UJ}$ & $0.40 \mathrm{wJ}$ & $0.40 \mathrm{UJ}$ & $0.40 \mathrm{us}$ & $2.00 \mathrm{UJ}$ & $0.60 \mathrm{UJ}$ \\
\hline Vanediun & $1.00 \mathrm{~J}$ & $0.90 \mathrm{~J}$ & $0.94 \mathrm{~J}$ & $1.10 \mathrm{~J}$ & 1.10 .1 & $1.60 \mathrm{~J}$ & $1.30 \mathrm{~J}$ & $1.70 \mathrm{~d}$ \\
\hline 2 ine & $148.00 \mathrm{~J}$ & $143.00 \mathrm{~J}$ & $156.00 \mathrm{~J}$ & $233.00 \mathrm{~J}$ & $106.00 \mathrm{~J}$ & $139.00 \mathrm{~J}$ & $468.00 \mathrm{~J}$ & $398.00 \mathrm{~J}$ \\
\hline Crenide & MR & $M R$ & Wh & NR & NR & NR & NR & MR \\
\hline C8.137 & $<1.049 \mathrm{UJ}$ & $01.046 \mathrm{UJ}$ & $<.9581$ us & $<1.263$ & $<.4480 \mathrm{UJ}$ & $\times .3427$ UJ & 82.798 Ud & $\$ 1.286$ UJ \\
\hline $8 r \cdot 90$ & $2.40 \mathrm{~J}$ & $0.01 R$ & $-0.60 \mathrm{UJ}$ & $0.33 \mathrm{~J}$ & 0.06 UJ & $0.70 \mathrm{~J}$ & $0.43 \mathrm{~J}$ & $0.30 \mathrm{UJ}$ \\
\hline Tc- 99 & $0.70 \mathrm{~J}$ & $1.40 \mathrm{~J}$ & $0.20 \mathrm{UJ}$ & $0.01 \mathrm{uJ}$ & $0.10 \mathrm{us}$ & $2.00 \mathrm{~J}$ & $0.70 \mathrm{R}$ & $0.10 \mathrm{UJ}$ \\
\hline
\end{tabular}

NOTE: All metals are reported in mo/kg. nedionuclides ore reported in pci/o.

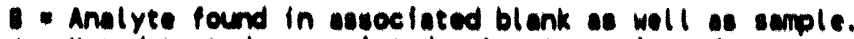

$J$ - Mot dotectedi essociated value is estimated.

NR - Nonreportable.

- Date ore unuseble.

$U$ - Not detected; value reported is semple quentitation limit.

UJ * Not dotected; may not accurately reflect semple quantitation $1 /$ mit.

ouplicate sample taken. 
Table D-2. Tree Leaf Results Collected in July 1992 at 100 Area Reactor Sites. (sheet 1 of 2)

\begin{tabular}{|c|c|c|c|c|c|c|}
\hline \multirow{3}{*}{ Const i tuent } & \multicolumn{6}{|c|}{ Reactor site } \\
\hline & Below F & Below F & Below $\mathrm{H}$ & Below H & Below H & Above $\mathrm{H}$ \\
\hline & $806 N 49$ & $806 N 50$ & $\begin{array}{l}\text { B06N51 } \\
\text { (B06N52) }\end{array}$ & $\begin{array}{c}\text { B06N52 } \\
\text { Dupl i cate }\end{array}$ & $806 N 53$ & B06N54 \\
\hline Aluminum & $58.40 \mathrm{~B}$ & $45.60 \mathrm{~B}$ & $59.60 \mathrm{~B}$ & $38.80 \mathrm{~B}$ & $43.40 \mathrm{~B}$ & $40.80 \mathrm{~B}$ \\
\hline Ant imony & $13.80 \mathrm{U}$ & $8.10 \mathrm{U}$ & $9.20 \mathrm{U}$ & $9.30 \mathrm{U}$ & $8.70 \mathrm{U}$ & $8.40 \mathrm{U}$ \\
\hline Arsenic & $3.30 \mathrm{~J}$ & $1.60 \mathrm{~J}$ & $1.70 \mathrm{~J}$ & $2.10 \mathrm{~J}$ & $1.90 \mathrm{~J}$ & $2.10 \mathrm{~J}$ \\
\hline Barium & $17.50 \mathrm{~B}$ & $36.40 \mathrm{~B}$ & $38.40 \mathrm{~B}$ & $39.00 \mathrm{~B}$ & $32.30 \mathrm{~B}$ & $26.40 \mathrm{~B}$ \\
\hline Beryllium & $0.59 \mathrm{id}$ & $0.34 \mathrm{U}$ & $0.39 \mathrm{U}$ & $0.40 \mathrm{U}$ & $0.57 \mathrm{U}$ & $0.35 \mathrm{U}$ \\
\hline Cactmiun & $1.30 \mathrm{U}$ & $0.73 \mathrm{U}$ & $0.83 U$ & $0.85 \mathrm{U}$ & $0.79 \mathrm{U}$ & $0.76 \mathrm{U}$ \\
\hline Calcium & 19700.00 & 24700.00 & 24400.00 & 25100.00 & 21500.00 & 26200.00 \\
\hline Chromium & $4.10 \mathrm{U}$ & $2.40 \mathrm{U}$ & $2.70 \mathrm{U}$ & $2.80 \mathrm{U}$ & $2.60 \mathrm{U}$ & $2.50 \mathrm{U}$ \\
\hline Cobsalt & $2.90 \mathrm{U}$ & $1.70 \mathrm{U}$ & $1.90 \mathrm{U}$ & $2.00 \mathrm{U}$ & $1.80 \mathrm{U}$ & $1.80 \mathrm{U}$ \\
\hline Copper & 25.20 & $9.20 \mathrm{~B}$ & $6.70 \mathrm{~B}$ & $8.10 \mathrm{~B}$ & $4.40 \mathrm{~B}$ & $6.50 \mathrm{~B}$ \\
\hline Iron & $206.00 \mathrm{~J}$ & $120.00 \mathrm{~J}$ & $136,00 \mathrm{~J}$ & $118.00 \mathrm{~J}$ & $108.00 \mathrm{~J}$ & $140.00 \mathrm{~J}$ \\
\hline Lead & 3.00 & 2.70 & 1.70 & 2.10 & $1.50 \mathrm{U}$ & 1.60 \\
\hline Magnes ium & 4590.00 & 3710.00 & 3190.00 & 3150.00 & 3260.00 & 5500.00 \\
\hline Manganese & 34.30 & 17.50 & 30.60 & 28.00 & 19.30 & 69.90 \\
\hline Mercury & $0.71 \mathrm{~J}$ & $0.32 \mathrm{~J}$ & $1.40 \mathrm{~J}$ & $0.59 \mathrm{~J}$ & $0.58 \mathrm{~J}$ & $0.57 \mathrm{~J}$ \\
\hline Nickel & $5.00 \mathrm{U}$ & $2.90 \mathrm{U}$ & $3.30 \mathrm{U}$ & $3.40 \mathrm{U}$ & $3.20 \mathrm{U}$ & $3.00 \mathrm{U}$ \\
\hline Potassium & 25900.00 & 16000.00 & 16600.00 & 16300.00 & 18000.00 & 10700.00 \\
\hline Selenium & 4.70 & $2.70 \mathrm{~J}$ & 2.80 & $2.60 \mathrm{~B}$ & $2.40 \mathrm{~B}$ & $1.90 \mathrm{~J}$ \\
\hline silver & $4.00 \mathrm{U}$ & $2.30 \mathrm{U}$ & $2.70 \mathrm{U}$ & $2.70 \mathrm{U}$ & $2.50 \mathrm{U}$ & $2.40 \mathrm{U}$ \\
\hline Sodium & $320.00 \mathrm{~B}$ & $230.00 \mathrm{~B}$ & $171.00 \mathrm{U}$ & $199.00 \mathrm{U}$ & $168.00 \mathrm{U}$ & $198.00 \mathrm{U}$ \\
\hline Thallium & $3.50 \mathrm{UJ}$ & $2.20 \mathrm{U}$ & $2.40 \mathrm{U}$ & $2.40 \mathrm{U}$ & $2.30 \mathrm{U}$ & $2.10 \mathrm{U}$ \\
\hline Venadium & $2.80 \mathrm{U}$ & $1.60 \mathrm{U}$ & $1.80 \mathrm{U}$ & $1.90 \mathrm{U}$ & $1.70 \mathrm{U}$ & $1.70 \mathrm{U}$ \\
\hline Zine & $49.60 \mathrm{~J}$ & $36.70 \mathrm{~J}$ & $29.80 \mathrm{~J}$ & $23.70 \mathrm{~J}$ & $17.30 \mathrm{~J}$ & $33.40 \mathrm{~J}$ \\
\hline Cyanide & MR & NR & NR & NR & NR & NR \\
\hline $\mathrm{Cs}-137$ & $\mathbf{u}$ & u & $u$ & $u$ & $U$ & U \\
\hline$S r-90$ & $-0.007 R$ & 0.340 & 0.330 & 0.001 & 2.600 & $-0.390 R$ \\
\hline Tritiun & -0.046 & 0.071 & .0 .005 & -0.038 & -0.038 & 0.320 \\
\hline
\end{tabular}

NOTE: All metals are reported in $\mathrm{mg} / \mathrm{kg}$. Radionuclides are reported in $\mathrm{pCi} / \mathrm{gm}$.

$B$ = Analyte found in associated blank as well as sample.

$J=$ Not detected, associated value is estimated.

NR = Nonreportable.

$R=$ Date are unusable.

$U=$ Not detected, value reported is sample quantitation limit.

UJ = Not detected, may not accurately reflect sample quantitation limit.

Duplicate sample taken. 
Table D-2. Tree Leaf Results Collected in July 1992 at 100 Area Reactor Sites. (sheet 2 of 2 )

\begin{tabular}{|c|c|c|c|c|c|c|c|c|}
\hline \multirow{3}{*}{ Const i tuent } & \multicolumn{8}{|c|}{ Reactor site } \\
\hline & Below D & Below D & Above D & Below K & Above $K$ & Below BC & Above BC & Control \\
\hline & $806 \times 55$ & $806 N 56$ & B06N57 & B06N58 & B06N59 & $806 N 60$ & B06N61 & $806 N 62$ \\
\hline Aluminum & $26.10 \mathrm{~B}$ & $47.20 \mathrm{~B}$ & $41.70 \mathrm{~B}$ & 105.00 & $35.50 \mathrm{~B}$ & $67.00 \mathrm{~B}$ & $49.90 \mathrm{~B}$ & 161.00 \\
\hline Ant imony & $8.30 \mathrm{U}$ & $9.50 \mathrm{U}$ & $7.10 \mathrm{U}$ & $8.50 \mathrm{U}$ & $9.00 \mathrm{U}$ & $8.90 \mathrm{U}$ & $8.40 \mathrm{U}$ & $9.30 \mathrm{U}$ \\
\hline Arsenic & $1.30 \mathrm{UJ}$ & $1.60 \mathrm{~J}$ & $1.40 \mathrm{~J}$ & $2.10 \mathrm{~J}$ & $2.40 \mathrm{~J}$ & $1.40 \mathrm{UJ}$ & $1.70 \mathrm{~J}$ & $1.60 \mathrm{~J}$ \\
\hline Barium & $24.30 \mathrm{~B}$ & $26.90 \mathrm{~B}$ & $36.80 \mathrm{~B}$ & $14.10 \mathrm{~B}$ & $64.30 \mathrm{~B}$ & $35.30 \mathrm{~B}$ & $40.70 \mathrm{~B}$ & $31.50 \mathrm{~B}$ \\
\hline Berylliun & $0.35 U$ & $0.40 \mathrm{U}$ & $0.30 \mathrm{U}$ & $0.36 \mathrm{U}$ & $0.38 \mathrm{U}$ & $0.38 \mathrm{~V}$ & $0.36 \mathrm{U}$ & $0.39 \mathrm{U}$ \\
\hline Cachium & $0.76 \mathrm{U}$ & $0.86 \mathrm{U}$ & $0.64 \mathrm{U}$ & $0.77 \mathrm{U}$ & $0.82 \mathrm{U}$ & $0.81 \mathrm{U}$ & $0.77 \mathrm{U}$ & $0.84 \mathrm{U}$ \\
\hline Calcium & 7500.00 & 18600.00 & 18800.00 & 21700.00 & 35700.00 & 25100.00 & 28900.00 & 22500.00 \\
\hline Chromiun & $2.50 \mathrm{U}$ & 3.90 & $3.20 \mathrm{~J}$ & 4.10 & 3.40 & $2.70 \mathrm{U}$ & $2.50 \mathrm{U}$ & 4.20 \\
\hline Cobalt & $1.80 \mathrm{U}$ & $2.00 \mathrm{U}$ & $1.50 \mathrm{U}$ & $1.80 \mathrm{U}$ & $1.90 \mathrm{U}$ & $1.90 \mathrm{U}$ & $1.80 \mathrm{U}$ & $2.00 \mathrm{U}$ \\
\hline Copper & $6.10 \mathrm{~B}$ & $7.80 \mathrm{~B}$ & $5.50 \mathrm{~B}$ & $8.10 \mathrm{~B}$ & $5.80 \mathrm{~B}$ & $4.50 \mathrm{~B}$ & $6.60 \mathrm{~B}$ & $6.80 \mathrm{~B}$ \\
\hline Iron & $89.40 \mathrm{~J}$ & $102.00 \mathrm{~J}$ & $109.00 \mathrm{~J}$ & $203.00 \mathrm{~J}$ & $148.00 \mathrm{~J}$ & $138.00 \mathrm{~J}$ & $147.00 \mathrm{~J}$ & $321.00 \mathrm{~J}$ \\
\hline Lead & 2.00 & 2.80 & 1.70 & 1.60 & $1.50 \mathrm{U}$ & $1.40 \mathrm{U}$ & 1.80 & 3.10 \\
\hline Magnesium & 4190.00 & $1940.00 \mathrm{~B}$ & 4530.00 & 3180.00 & 3190.00 & $1830.00 \mathrm{~B}$ & 4690.00 & 3030.00 \\
\hline Manganese & 9.40 & 22.30 & 25.00 & 32.70 & 24.00 & 23.60 & 36.50 & 20.40 \\
\hline Mercury & $0.31 \mathrm{~J}$ & $0.67 \mathrm{~J}$ & $2.00 \mathrm{~J}$ & $1.40 \mathrm{~J}$ & $1.40 \mathrm{~J}$ & $2.70 \mathrm{~J}$ & $0.38 \mathrm{~J}$ & $0.98 \mathrm{~J}$ \\
\hline Nickel & $3.00 \mathrm{U}$ & $5.10 \mathrm{~B}$ & $2.60 \mathrm{U}$ & $3.10 \mathrm{U}$ & $3.30 \mathrm{U}$ & $3.20 \mathrm{U}$ & $3.10 \mathrm{U}$ & $3.40 \mathrm{U}$ \\
\hline Potassium & 4600.00 & 17500.00 & 13300.00 & 15900.00 & 15800.00 & 18400.00 & 14400.00 & 17800.00 \\
\hline solenium & $2.30 \mathrm{~B}$ & $2.20 \mathrm{~B}$ & $2.00 \mathrm{~B}$ & $1.90 \mathrm{UJ}$ & $1.90 \mathrm{Ud}$ & $2.50 \mathrm{~B}$ & $1.90 \mathrm{UJ}$ & $2.30 \mathrm{~B}$ \\
\hline silver & $2.40 \mathrm{U}$ & $2.80 \mathrm{U}$ & $2.10 \mathrm{U}$ & $2.50 \mathrm{U}$ & $2.60 \mathrm{U}$ & $2.60 \mathrm{U}$ & $2.50 \mathrm{U}$ & $2.70 \mathrm{U}$ \\
\hline Sodium & $185.00 \mathrm{U}$ & $173.00 \mathrm{U}$ & $191.00 \mathrm{U}$ & $156.00 \mathrm{U}$ & $167.00 \mathrm{U}$ & $220.00 \mathrm{U}$ & $155.00 \mathrm{U}$ & $182.00 \mathrm{U}$ \\
\hline rhallium & $2.20 \mathrm{U}$ & $2.40 \mathrm{U}$ & $1.90 \mathrm{U}$ & $2.20 \mathrm{U}$ & $2.30 \mathrm{U}$ & $2.30 \mathrm{U}$ & $2.20 \mathrm{U}$ & $2.40 \mathrm{U}$ \\
\hline Vanadium & $1.70 \mathrm{U}$ & $1.90 \mathrm{U}$ & $1.40 \mathrm{U}$ & $1.70 \mathrm{U}$ & $1.80 \mathrm{U}$ & $1.80 \mathrm{U}$ & $1.70 \mathrm{U}$ & $1.90 \mathrm{U}$ \\
\hline 2 inc & $26.00 \mathrm{~J}$ & $35.00 \mathrm{~J}$ & $28.50 \mathrm{~J}$ & $18.80 \mathrm{~J}$ & $19.50 \mathrm{~J}$ & $22.20 \mathrm{~J}$ & $20.80 \mathrm{~J}$ & $19.40 \mathrm{~J}$ \\
\hline Cyanide & NR & NR & NR & NR & NR & NR & NR & NR \\
\hline Cs- 137 & U & $\mathbf{U}$ & $\mathbf{U}$ & $\mathbf{U}$ & $\mathbf{U}$ & U & U & $U$ \\
\hline $5 r-90$ & 0.230 & $0.260 \mathrm{R}$ & 0.270 & 35.00 & -0.330 & $6.800 \mathrm{R}$ & 0.180 & 0.150 \\
\hline Tritium & -0.063 & 0.640 & -0.018 & 0.730 & 0.005 & 3.600 & 2.100 & 0.014 \\
\hline
\end{tabular}

NOTE: All metals are reported in $\mathrm{mg} / \mathrm{kg}$. Radionuclides are reported in $\mathrm{pCi} / \mathrm{gm}$.

\footnotetext{
B - Analyte found in associated blank as well as sample.

$J=$ Not detected, associated value is estimated.

NR = Nonreportable.

$R=$ Date ore unusable.

$U=$ Not detected, value reported is sample quantitation limit

$U J=$ Not detected, may not accurately reflect sample quantitation limit.

Duplicate sample taken.
} 
Table D-3. Tree Leaf Results Collected in October 1992.

(Part I)

\begin{tabular}{|c|c|c|c|c|c|c|}
\hline \multirow[b]{2}{*}{ Const i tuent } & \multicolumn{6}{|c|}{ Semple Madia, Locations, and Sample Numbers } \\
\hline & $\begin{array}{c}\text { Llmbs } \\
\text { Below sc } \\
\text { Bo7925 }\end{array}$ & $\begin{array}{l}\text { Leaves } \\
\text { Below BC } \\
\text { Bo7926 }\end{array}$ & $\begin{array}{l}\text { Leaves } \\
\text { Below BC } \\
\text { Bo7927 }\end{array}$ & $\begin{array}{c}\text { Limbs } \\
\text { Below BC } \\
\text { Bo7928 }\end{array}$ & $\begin{array}{c}\text { Limbe } \\
\text { Above K } \\
\text { Bo7930 }\end{array}$ & $\begin{array}{l}\text { Leaves } \\
\text { Above K } \\
\text { so7931 }\end{array}$ \\
\hline Cs-137 & U & $\mathbf{u}$ & $\mathbf{u}$ & $\mathbf{u}$ & $u$ & $u$ \\
\hline $9 r-90$ & $9.90 \mathrm{~J}$ & $23.00 \mathrm{~J}$ & $13.00 \mathrm{~J}$ & $7.10 \mathrm{~J}$ & $-0.37 \mathrm{UJ}$ & 0.16 UJ \\
\hline Tritiun & $3.0 \mathrm{~J}$ & $1.2 \mathrm{~J}$ & $1.0 \mathrm{~J}$ & $3.8 \mathrm{~J}$ & $0.34 \mathrm{~J}$ & $0.26 \mathrm{~J}$ \\
\hline
\end{tabular}

Table D-3. Tree Leaf Results Collected in October 1992. (Part II)

\begin{tabular}{|c|c|c|c|c|c|c|}
\hline \multirow[b]{2}{*}{ Conse I tuent } & \multicolumn{6}{|c|}{ Semple Media, Locations, and Semple Numbers } \\
\hline & $\begin{array}{l}\text { Limbs } \\
\text { Above } K \\
\text { so7932 }\end{array}$ & $\begin{array}{l}\text { Leaves } \\
\text { Above K } \\
\text { Bo7933 }\end{array}$ & $\begin{array}{l}\text { Limbs } \\
\text { Below K } \\
\text { Bo7929 }\end{array}$ & $\begin{array}{l}\text { Leaves } \\
\text { Below K } \\
\text { Bo7934 }\end{array}$ & $\begin{array}{l}\text { Limbs } \\
\text { Below } K \\
\text { Bo7935 }\end{array}$ & $\begin{array}{l}\text { Leaves } \\
\text { Below K } \\
\text { Bo7936 }\end{array}$ \\
\hline C8-137 & $\mathbf{u}$ & $u$ & $u$ & $u$ & U & u \\
\hline $8 r-90$ & $0.068 \mathrm{UJ}$ & 0.35 UJ & $43.00 \mathrm{~J}$ & $88.00 \mathrm{~J}$ & $-0.16 \mathrm{UJ}$ & $0.55 \mathrm{UJ}$ \\
\hline Tritiun & $0.34 \mathrm{~J}$ & $0.12 \mathrm{~J}$ & $0.39 \mathrm{~J}$ & $0.26 \mathrm{~J}$ & $1.8 \mathrm{~J}$ & $0.75 \mathrm{~J}$ \\
\hline
\end{tabular}
pci/era.

NOTE: All metals are reported in $\mathrm{mg} / \mathrm{kg}$. Radionuclldes are reported in

B analyte found in associated blank as well as sample.

$\checkmark$. Mot detected; sseociated value is estimated.

NR = Nonreportable.

$R$ - Data unusable.

$U$ = Not detected; value reported is samole quantitation limit.

UJ - Not detected; may not accurately reflect semple quentitation limit. 
WHC-EP-0620

This page intentionally left blank. 
WHC-EP-0620

\section{APPENDIX E}

ANALYTICAL RESULTS FOR COYOTES AND RAPTORS 
This page intentionally left blank. 
Table E-1. Coyote Results from 1992. (sheet 1 of 2)

\begin{tabular}{|c|c|c|c|c|c|c|}
\hline \multirow{2}{*}{ Analyte } & \multicolumn{6}{|c|}{ Semple Locations and Identiffection Numbers } \\
\hline & $\stackrel{0}{108400}$ & $\stackrel{0}{D}{ }_{\text {BOSND1 }}$ & $\stackrel{0}{806 N D 7}$ & Dosnd8 & SOSNFo & 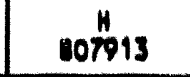 \\
\hline Aluminum & $2000.00 \mathrm{~J}$ & $1020.00 \mathrm{~J}$ & $760.00 \mathrm{~J}$ & $905.00 \mathrm{~J}$ & $1980.00 \mathrm{~J}$ & 1950.00 \\
\hline Ant Imeny & $3.60 \mathrm{UJ}$ & $3.30 \mathrm{UJ}$ & $3.60 \mathrm{UJ}$ & 3.30 Ud & $3.30 \mathrm{UJ}$ & $3.60 \mathrm{UJ}$ \\
\hline Arsente & $2.90 \mathrm{R}$ & $2.90 \mathrm{R}$ & $3.00 R$ & $2.90 \mathrm{R}$ & $3.10 n$ & $3.40 \mathrm{UJ}$ \\
\hline Sarín & 53.10 & 27.40 & 53.50 & 22.00 & $61.30 \mathrm{~B}$ & $33.90 \mathrm{U}$ \\
\hline Derylllum & $0.09 \mathrm{U}$ & $0.09 B$ & $0.09 \mathrm{U}$ & $0.08 \mathrm{U}$ & $0.08 \mathrm{U}$ & $0.27 \mathrm{U}$ \\
\hline Cocinlun & $0.73 \mathrm{U}$ & $0.20 \mathrm{U}$ & $0.58 \mathrm{u}$ & $0.36 \mathrm{U}$ & $0.21 \mathrm{U}$ & 4.10 \\
\hline Colelum & $07300.00 \mathrm{~J}$ & $12000.00 \mathrm{~J}$ & $162000.00 \mathrm{~J}$ & $33800.00 \mathrm{~J}$ & $111000.00 \mathrm{~J}$ & 156000.00 \\
\hline Chromilu: & 6.50 & $1.30 \mathrm{~V}$ & 4.00 & 2.80 & 5.00 & 7.10 \\
\hline Cobolt & 1.801 & $1.50 \mathrm{~V}$ & $0.57 \cup$ & 0.81 & $1.70 \mathrm{~s}$ & $1.30 \mathrm{~B}$ \\
\hline Copper & 15.20 & 16.30 & 12.00 & 12.50 & 17.50 & 13.90 \\
\hline Iron & $3450.00 \mathrm{~J}$ & $4630.00 \mathrm{~J}$ & $1660.00 \mathrm{~J}$ & $2420.00 \mathrm{~J}$ & $4000.00 \mathrm{~J}$ & 2930.00 \\
\hline lead & 11.50 & 1.60 & 13.90 & 5.60 & 5.70 & $7.50 \mathrm{n}$ \\
\hline Maenestiven & 660.00 & $\$ 100.00$ & $4360.00 \mathrm{~J}$ & 1500.00 & 5880.00 & 6950.00 \\
\hline Menocnese & $196.00 \mathrm{~J}$ & $0.10 \mathrm{~J}$ & 152.00 & $60.30 \mathrm{~J}$ & $02.60 \mathrm{~J}$ & 107.00 \\
\hline Mereury & Nh & NR & Mn & NR & Nh & Nh \\
\hline Mickel & 3.30 & $4.00 \mathrm{n}$ & 1.408 & 2.300 & 3.30 & 2.700 \\
\hline Potenolun & $2090.00 \mathrm{~J}$ & $0350.00 \mathrm{~J}$ & $2600.00 \mathrm{~J}$ & $1790.00 \mathrm{~J}$ & $967.00 \mathrm{~J}$ & 2510.00 \\
\hline saleniun & $3.70 \mathrm{R}$ & $3.80 n$ & $4.00 \mathrm{R}$ & $3.80 \mathrm{n}$ & $4.00 R$ & $4.20 \mathrm{UJ}$ \\
\hline sllver & $0.53 \mathrm{U}$ & $0.49 \mathrm{U}$ & $0.53 \mathrm{U}$ & $0.52 u$ & $0.52 \mathrm{U}$ & $0.56 \mathrm{U}$ \\
\hline sodiun & $697.00 \mathrm{~J}$ & $2070.00 \mathrm{~J}$ & $4370.00 \mathrm{~J}$ & $743.00 \mathrm{~J}$ & $798.00 \mathrm{~J}$ & $3030.00 \mathrm{~J}$ \\
\hline Tholllum & 0.80 & $0.26 \mathrm{U}$ & $0.27 \mathrm{~V}$ & $0.26 \mathrm{U}$ & $0.27 \mathrm{U}$ & $0.82 \mathrm{UJ}$ \\
\hline Venadiun & 10.20 & 5.600 & 3.601 & 4.200 & 8.301 & $6.50 \triangle$ \\
\hline 2 lne & 240.00 & 66.30 & 294.00 & 282.00 & 295.00 & 367.00 \\
\hline Crenida & NR & Mn & NR & NR & Nh & Nn \\
\hline Co.137 & $u$ & $u$ & 0.44 & $u$ & $u$ & u \\
\hline $8 r \cdot 90$ & $\cdot 0.39$ & 0.30 & 0.073 & 0.15 & 4.50 & 0.29 \\
\hline
\end{tabular}

NOTf: All motels ore reported in ma/kg. Radionucllidas are reported in pcl/om.

- Anolvete found In assoclated blank as well as sanple.

$J$ - Not detected; aseocioted value is estimated.

NR - Nonreporteble.

- Doto are unueble.

$U$ - Mot dotected; value reported is eample quantitation IImitt.

UJ - Not detected; may not accurately reflect semple quantitotion limit. 
Table E-1. Coyote Results from 1992. (sheet 2 of 2)

\begin{tabular}{|c|c|c|c|c|c|}
\hline \multirow{2}{*}{ Anolvee } & \multicolumn{5}{|c|}{ Semplo Locetions and Identiflention Mubers } \\
\hline & $\stackrel{1}{10 \% 17}$ & $\begin{array}{l}\text { Conerol } \\
\text { losinoz }\end{array}$ & $\begin{array}{l}\text { Control } \\
\text { Dos4os }\end{array}$ & $\begin{array}{l}\text { Control } \\
\text { losups }\end{array}$ & $\begin{array}{l}\text { Centrol } \\
\text { Dosnos }\end{array}$ \\
\hline Aluainu & 2340.00 & $3970.00 \mathrm{~J}$ & $2480.00 \mathrm{~J}$ & $3040.00 \mathrm{~J}$ & $2330.00 \mathrm{~J}$ \\
\hline Ane Imeny & $3.60 \mathrm{UJ}$ & $3.50 \mathrm{UJ}$ & $3.40 \mathrm{uJ}$ & $3.30 \mathrm{Ud}$ & $3.30 \mathrm{UJ}$ \\
\hline Arsenle & $3.40 \mathrm{uJ}$ & $2.90 \mathrm{n}$ & $3.00 R$ & $3.00 \mathrm{n}$ & $2.00 \mathrm{n}$ \\
\hline earlu & 6.90 & 69.80 & 55.50 & $38.90 \div$ & 92.70 \\
\hline Derylliv & $0.32 \mathrm{~V}$ & $0.08 v$ & $0.08 \mathrm{U}$ & $0.08 \mathrm{U}$ & $0.08 \mathrm{U}$ \\
\hline coculun & 1.10 & $0.20 \mathrm{U}$ & $0.20 \mathrm{~V}$ & $0.21 \mathrm{v}$ & $0.20 \mathrm{~J}$ \\
\hline Calolu & 95000.00 & $37500.00 \mathrm{~J}$ & $73300.00 \mathrm{~J}$ & $66000.00 \mathrm{~J}$ & 22300.00 \\
\hline Chroalum & 3.90 & 3.40 & 4.90 & 3.30 & 3.90 \\
\hline Cobale & 1.001 & 4.70 & 2.70 & 3.001 & 1.601 \\
\hline Coppor & 22.00 & 11.30 & 18.10 & 13.10 & 21.20 \\
\hline Iren & 480.00 & $11300.00 \mathrm{~J}$ & $3090.00 \mathrm{~J}$ & $0110.00 \mathrm{~J}$ & $4300.00 \mathrm{~J}$ \\
\hline Loed & $9.00 \mathrm{n}$ & 2.30 & 2.90 & 3.70 & 3.80 \\
\hline nognosivn & 5990.00 & 4020.00 & 4430.00 & 3330.00 & 4260.00 \\
\hline Mengeneve & 261.00 & $206.00 \mathrm{~J}$ & $116.00 \mathrm{~J}$ & $109.00 \mathrm{~d}$ & $123.00 \mathrm{~J}$ \\
\hline Mereury & Nn & Nn & Mn & nn & $m n$ \\
\hline Mlokel & 3.600 & $6.90 \%$ & 3.001 & $6.90 \mathrm{~B}$ & 3.201 \\
\hline Potansive & 2210.00 & $1670.00 \mathrm{~J}$ & $2570.00 \mathrm{~J}$ & $011.00 \mathrm{~J}$ & $6000.00 \mathrm{~J}$ \\
\hline selenlum & $6.10 \mathrm{ud}$ & $3.80 \mathrm{n}$ & $3.90 \mathrm{R}$ & $3.90 \mathrm{R}$ & $3.70 \mathrm{n}$ \\
\hline sllver & $0.53 \mathrm{~V}$ & $0.31 \mathrm{u}$ & $0.31 v$ & $0.91 \mathrm{v}$ & $0.50 \mathrm{~V}$ \\
\hline Sodiun & $2320.00 \mathrm{~J}$ & $662.00 \mathrm{~d}$ & $6500.00 \mathrm{~J}$ & $674.00 \mathrm{~J}$ & $1640.00 \mathrm{~J}$ \\
\hline Thollium & $0.80 \mathrm{UJ}$ & $0.26 \mathrm{U}$ & $0.27 v$ & $0.27 \mathrm{U}$ & $0.25 v$ \\
\hline Venudlua & 9.701 & 22.40 & 10.30 & 11.60 & 7.600 \\
\hline 2 ine & 295.00 & 117.00 & 206.00 & 70.60 & 176.00 \\
\hline Gyenles & min & Nh & Mn & Nh & $\mathrm{Nh}$ \\
\hline $\mathrm{Ce} \cdot 137$ & $u$ & $u$ & $u$ & $u$ & $u$ \\
\hline $9 r=90$ & $0.44 \mathrm{R}$ & 0.21 & 0.11 & 0.00 & 0.006 \\
\hline
\end{tabular}

Nore: All matele are reported in mo/kg. Radionuclidas are reported in pel/am.

- Anilye found in aseoclated blank os woll we smple.

$j$ - Mot detectedi aseoclated volue lo estimated.

NR - Menreportable.

- Dete ore unusible.

$u=$ - Not detected; valius reported is semple quantitation IIrit.

UJ - Not detectedi moy not sceurotoly reflect semple quentitation IImit. 
Table E-2. Raptor Results from 1992.

\begin{tabular}{|c|c|c|c|c|c|c|c|}
\hline \multirow{2}{*}{ Cenot levent } & \multicolumn{7}{|c|}{ semple locotions and Identiflestion Numbers } \\
\hline & coswos & 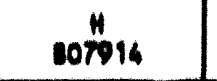 & 207915 & $10 \% 916$ & $\begin{array}{l}\text { Control } \\
\text { sorisio }\end{array}$ & $\begin{array}{l}\text { Centrol } \\
\text { cosnos }\end{array}$ & $\begin{array}{l}\text { Conirol } \\
\text { coswos }\end{array}$ \\
\hline Aluinum & $905.00 \mathrm{~J}$ & 035.00 & 834.00 & 1210.00 & 1170.00 & $1950.00 \mathrm{~J}$ & $3040.00 \mathrm{~J}$ \\
\hline Ant Imany & 3.30 Ud & 3.80 UN & 3.70 us & 3.60 Wd & $3.00 \mathrm{Ud}$ & $3.50 \mathrm{UJ}$ & 3.50 WJ \\
\hline Arsenle & $2.90 \times$ & $3.60 \mathrm{U}$ & $3.40 \mathrm{UJ}$ & 3.90 ud & 3.50 Ud & $2.90:$ & $3.00 \cap$ \\
\hline Dorlum & 22.000 & 90.20 & 74.10 & 61.10 & $\$ 3.40$ & 49.10 & 39.300 \\
\hline Cervillon & $0.00 \mathrm{u}$ & $0.14 \mathrm{v}$ & $0.09 \mathrm{v}$ & $0.27 \cup$ & $0.26 \mathrm{v}$ & $0.08 v$ & $0.00 \mathrm{v}$ \\
\hline cectulu & $0.30 \mathrm{~V}$ & $0.91 \mathrm{u}$ & $0.22 v$ & $0.22 \mathrm{v}$ & $0.22 v$ & $0.21 v$ & $0.21 \mathrm{v}$ \\
\hline coleiven & $38500.00 \mathrm{~J}$ & 104000.00 & 114000.00 & 116000.00 & 163000.00 & 02200.00 & $46000.00 \mathrm{~J}$ \\
\hline chromilu & 2.80 & 9.00 & 3.30 & 3.70 & 6.10 & 3.40 & $\$ .30$ \\
\hline Cobale & 0.010 & 0.50 & 0.21 & 0.601 & 0.970 & $1.30 \mathrm{\theta}$ & 3.001 \\
\hline Cepoer & 12.30 & 3.90 & 20.20 & 16.40 & 18.90 & 20.50 & 13.10 \\
\hline Iren & $2420.00 \mathrm{~J}$ & 1810.00 & 1960.00 & 2700.00 & 2330.00 & $3510.00 \mathrm{~J}$ & $6110.00 \mathrm{~d}$ \\
\hline Lead & 8.60 & $10.30 \mathrm{n}$ & $3.10 n$ & $4.00 n$ & $1.80 n$ & 2.60 & 3.70 \\
\hline nocrestive & 1900.00 & 3010.00 & 3890.00 & 3290.00 & $\$ 040.00$ & 2600.00 & 3330.00 \\
\hline Mennenceses & $60.30 \mathrm{~d}$ & 639.00 & 37.00 & 76.50 & 69.90 & $93.00 \mathrm{~J}$ & $109.00 \mathrm{~J}$ \\
\hline Mroum & $m$ & mn & Mn & Mn & Mn & Mh & wh \\
\hline NIckel & $2.30 \mathrm{~B}$ & $1.20 \mathrm{v}$ & $1.20 \mathrm{~B}$ & 1.000 & 1.408 & $3.20 \mathrm{~s}$ & 4.900 \\
\hline Potensin & $1700.00 \mathrm{~J}$ & 1680.00 & 1120.00 & 1120.00 & 1930.00 & $1940.00 \mathrm{~d}$ & $811.00 \mathrm{~J}$ \\
\hline solenicu & $3.80 \mathrm{n}$ & $6.60 \mathrm{UJ}$ & $4.20 \mathrm{UN}$ & $6.20 \mathrm{UJ}$ & 4.20 UJ & $3.70 \mathrm{R}$ & $3.60 ?$ \\
\hline sllver & $0.52 \mathrm{~V}$ & $0.36 \mathrm{~V}$ & $0.93 \mathrm{U}$ & $0.54 \mathrm{v}$ & $0.56 \mathrm{U}$ & $0.52 \mathrm{U}$ & $0.51 \mathrm{U}$ \\
\hline sediven & $743.00 \mathrm{~J}$ & $2110.00 \mathrm{~J}$ & $3060.00 \mathrm{~J}$ & $3980.00 \mathrm{~J}$ & $2510.00 \mathrm{~J}$ & $1390.00 \mathrm{~J}$ & $676.00 \mathrm{~J}$ \\
\hline Thollite & $0.26 \mathrm{~V}$ & $0.0 s$ Ud & $0.02 \mathrm{U}$ & $0.82 \mathrm{~V}$ & $0.12 \mathrm{v}$ & $0.26 \mathrm{~V}$ & $0.27 \mathrm{v}$ \\
\hline Venudive & 4.201 & 3.000 & 3.701 & $3.60 \mathrm{D}$ & 6.600 & 7.001 & 11.40 \\
\hline $2 \ln e$ & 222.00 & 305.00 & 248.00 & 266.00 & 307.00 & 267.00 & 70.60 \\
\hline cronles & $m n$ & $M n$ & Nn & Nn & Mn & nn & in \\
\hline $68 \cdot 137$ & $\mathbf{u}$ & $u$ & u & $u$ & $v$ & $u$ & $v$ \\
\hline $8 r \cdot 90$ & 0.19 & 0.10 & $6.70 \mathrm{~J}$ & 0.035 & $0.37 \mathrm{~J}$ & 0.76 & 0 \\
\hline
\end{tabular}

norti All entels ore reported in mo/kg. Redionuclldes are reported in $\mathrm{ecl} / \mathrm{cm}$.

- Anolve feund in esseclased blenk as wall os semple.

$J$ - Not detestedi useceloted volus lo estimated.

Mn - Nenreporteble.

- Dote ore umacible.

U - Not detectedi value reported is scmole quantitation IImit.

US - Not dateeted may not eceurately roflect eemple quantitation limit. 
WHC-EP-0620

\section{APPENOIX F}

\section{ANALYTICAL RESULTS FOR ANT MOUNDS}


WHC-EP-0620

This page intentionally left blank. 
WHC-EP-0620

Table F-1. Ant Mound Soll Results from 1992 at F Reactor.

\begin{tabular}{|c|c|c|c|c|c|}
\hline \multirow{3}{*}{ Conutiluent } & \multicolumn{5}{|c|}{ Ienple Identifleation Numere and Loestion } \\
\hline & $120 \cdot 1 \cdot 1$ & $126 \cdot 1 \cdot 1$ & $126 \cdot 1 \cdot 14$ & $118 \cdot 1 \cdot 2$ & Control \\
\hline & nowives & nosnos & cosineo & nocwne & 107010 \\
\hline Alualnan & 0160.00 & 0000.00 & 1020.00 & $\$ 950.00$ & 4860.00 \\
\hline MneInimy & $3.10 !$ & $3.30 \AA$ & 3.60 Ud & $3.30 \mathrm{WJ}$ & 3.10 Ud \\
\hline Arechle & 0.570 & 0.011 & $1.70 \mathrm{v}$ & 2.10 & 2.30 \\
\hline Sartur & 316.00 & 230.00 & 01.70 & 93.00 & $\mathbf{\omega} . \mathbf{3 0}$ \\
\hline cenvillin & $0.01 \mathrm{u}$ & $0.07 \mathrm{v}$ & 0.49 & 0.150 & $0.13 v$ \\
\hline centur & $0.10 \mathrm{u}$ & $0.20 u$ & $0.20 \mathrm{v}$ & $0.20 \mathrm{U}$ & $0.18 \mathrm{u}$ \\
\hline colelu & 020.00 & 2000.00 & 340.00 & 310.00 & 0110.00 \\
\hline enrealu & 0.40 & $\$ .30$ & 0.40 & 7.00 & 3.50 \\
\hline Gcoble & 7.001 & 0.608 & 7.401 & $7.30 \mathrm{~s}$ & 10.10 \\
\hline Comper & 13.50 & 12.30 & 13.60 & 10.60 & 12.60 \\
\hline Iren & 13200.00 & 15900.00 & 19500.00 & 19500.00 & 19000.00 \\
\hline lewd & $9.00 \mathrm{~J}$ & $3.40 \mathrm{~J}$ & 3.00 & 3.60 & $3.90 \mathrm{n}$ \\
\hline nemserion & $\$ 170.00$ & $\$ 410.00$ & 340.00 & 300.00 & 6020.00 \\
\hline Menerneses & 211.00 & 287.00 & 240.00 & 227.00 & 269.00 \\
\hline Mreury & $0.05 \mathrm{v}$ & $0.05 v$ & $0.04 \mathrm{~V}$ & $0.06 \mathrm{~V}$ & $0.09 u$ \\
\hline Nlekel & 8.20 & 7.10 & 0.40 & 0.701 & 6.500 \\
\hline Potcesolu & 1270.00 & 1200.00 & 1090.00 & 1230.00 & 90.00 \\
\hline Selenium & $0.7 \mathrm{WJ}$ & $0.75 \mathrm{WJ}$ & $0.71 \mathrm{VN}_{\mathrm{J}}$ & $0.69 \mathrm{U}$ & 0.60 us \\
\hline sllver & $0.69 \mathrm{U}$ & $0.6 \cup$ & $0.51 \mathrm{~V}$ & $0.69 \mathrm{U}$ & 0.01 \\
\hline sedium & $421.00 \mathrm{~J}$ & $503.00 \mathrm{~J}$ & $165.00 \mathrm{~J}$ & $132.00 \mathrm{~J}$ & $192.00 \mathrm{~J}$ \\
\hline Thollin & $0.7 \mathrm{v}$ & $0.5 \mathrm{v}$ & $0.26 \mathrm{v}$ & $0.25 \mathrm{U}$ & $0.60 \mathrm{~W}$ \\
\hline Venediun & 35.40 & 41.60 & 60.40 & 3.70 & 52.20 \\
\hline $81 n$ & 32.20 & 39.70 & 39.60 & 32.60 & 63.30 \\
\hline Eventes & Mn & Mn & Nn & mo & mn \\
\hline $60 \cdot 137$ & $\mathbf{u}$ & $u$ & 0.11 & $u$ & 0.071 \\
\hline $8 r-9$ & $\cdot 0.3$ & $\cdot 0.06$ & 0.36 & 0.23 & 0.36 \\
\hline
\end{tabular}

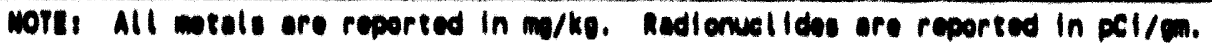

- - Anlyte tound in anseclated blenk as woll as semple.

$J$ - Mot deteeted asececloted value le eseimated.

un - Nenreporiedele.

- Dote ore unuscblo.

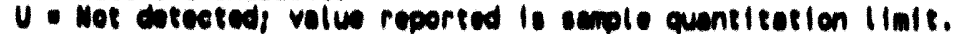

UJ - Not detectedi moy not eccurately reflect semple quantitotion IImite. 
Table F-2. Ant Mound Results From H Reactor Collected in 1992.

\begin{tabular}{|c|c|c|c|c|c|c|}
\hline \multirow{3}{*}{ cenvel teune } & \multicolumn{6}{|c|}{ suple Identifleation Wubars and Lecations } \\
\hline & $110 \cdot N \cdot 3$ & $118 \cdot N \cdot 3$ & $118 \cdot N \cdot 7$ & $118 \cdot n \cdot 7$ & $118 \cdot N \cdot 2$ & Control \\
\hline & cosing & cosuns & Iesures & cosing & rosucl & 107918 \\
\hline Aluinum & 370.00 & 5100.00 & 3430.00 & 3000.00 & 5270.00 & 4960.00 \\
\hline Ant I Eximy & $3.20 \mathrm{WJ}$ & $3.00 \mathrm{UN}$ & $3.10 \mathrm{ud}$ & $3.20 \mathrm{UJ}$ & 3.30 UJ & $3.10 \mathrm{UJ}$ \\
\hline Arsente & 2.30 & 4.00 & 4.80 & $1.30 \mathrm{U}$ & 2.60 & 2.30 \\
\hline Darlue & $\$ 1.00$ & 02.00 & 4.90 & 46.10 & 68.80 & 0.30 \\
\hline Derrillive & 0.350 & 0.420 & $0.32 \div$ & 0.34 & 0.350 & $0.13 u$ \\
\hline cectulu & $0.10 \mathrm{v}$ & $0.10 \mathrm{u}$ & $0.18 \mathrm{U}$ & $0.19 \mathrm{U}$ & $0.19 \mathrm{U}$ & $0.18 \mathrm{U}$ \\
\hline coletiv & 4030.00 & $3 \times 0.00$ & 3610.00 & 4360.00 & 3370.00 & 8100.00 \\
\hline Chremile & 9.70 & 6.90 & 3.40 & 4.70 & 7.80 & 5.30 \\
\hline Comale & 3.000 & 6.601 & 3.100 & $6.00 \mathrm{~B}$ & $0.00 \mathrm{O}$ & 10.10 \\
\hline Cequer & 10.60 & 12.00 & 13.00 & 12.70 & 10.30 & 12.60 \\
\hline Iren & 12000.00 & 14100.00 & 10700.00 & 12000.00 & 13900.00 & 19800.00 \\
\hline toed & 3.90 & 15.50 & 24.30 & $6.20 \mathrm{~J}$ & 12.20 & $3.90 n$ \\
\hline Matreselun & 3070.00 & 3200.00 & 2690.00 & 2020.00 & 3430.00 & $\$ 020.00$ \\
\hline Meneanives & 176.00 & 223.00 & 157.00 & 183,00 & 240.00 & 209.00 \\
\hline Mereury & $0.05 \mathrm{v}$ & $0.04 \mathrm{U}$ & $0.05 v$ & $0.04 \mathrm{U}$ & $0.05 \mathrm{U}$ & $0.05 v$ \\
\hline Mlakel & 0.70 & 6.00 & 9.008 & $3.30 \mathrm{~s}$ & 7.10 & 0.300 \\
\hline Poteses iv & 80.000 & 1370.00 & 705.00 & 899.001 & 1380.00 & 990.00 \\
\hline Seleniv & $0.0 \mathrm{~V}$ & $0.02 \mathrm{U}$ & 0.05 us & $0.7 \mathrm{v}$ & $0.4 \mathrm{~V}$ & $0.6 \mathrm{UN}$ \\
\hline Sllver & 0.40 & $0.46 \mathrm{U}$ & $0.67 \mathrm{~V}$ & $0.6 \mathrm{U}$ & $0.32 \mathrm{v}$ & 0.010 \\
\hline sediun & $112.00 \mathrm{~J}$ & $136.00 \mathrm{~J}$ & $135.00 \mathrm{~J}$ & $127.00 \mathrm{~J}$ & $115.00 \mathrm{~J}$ & $199.00 \mathrm{~J}$ \\
\hline Thollice & $0.23 \mathrm{~V}$ & $0.23 \mathrm{U}$ & $0.25 \mathrm{~V}$ & $0.26 \mathrm{~V}$ & $0.23 \mathrm{u}$ & $0.6 \mathrm{UJ}$ \\
\hline Venedice & 27.30 & 30.60 & 23.70 & 29.50 & 27.40 & 92.20 \\
\hline $21 \mathrm{ne}$ & 27.30 & 33.60 & 30.80 & 32.00 & 33.60 & 43.30 \\
\hline Crented & m & Mn & Wn & $M$ & Mn & Nn \\
\hline $60 \cdot 137$ & 0.34 & 0.19 & 0.26 & $u$ & 0.25 & 0.071 \\
\hline $9 n \cdot 80$ & 0.19 & 0.17 & 0.043 & 0.052 & 0.10 & 0.36 \\
\hline
\end{tabular}

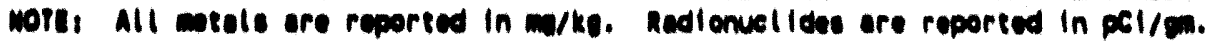

- Mnalre found in eaceelated blenk as well as semple.

$J$ - Mot detected, insoelated volue la estimated.

min - venroporteblo.

n. Deto ore unumble.

$U$ - Not dotected, volus reported to scmple quentitotion IIfitit.

WJ - Not dotected, may not securately reflect emple quantitation IImit. 
Table F-3. Ant Mound Results From D Reactor Collected in 1992.

\begin{tabular}{|c|c|c|c|c|c|}
\hline \multirow{3}{*}{ Conati tuent } & \multicolumn{5}{|c|}{ Semple Identifleation Munbers and Locations } \\
\hline & $196 \cdot 0 R \cdot 9$ & $116 \cdot 0 R \cdot 9$ & $116 \cdot 0 \cdot 6$ & $116 \cdot 0 \cdot 2$ & Conerol \\
\hline & sodvc2 & DOSNCG & DOSNC6 & nosnces & 807918 \\
\hline Alvinu & 4780.00 & 4220.00 & 5100.00 & 6470.00 & 4560.00 \\
\hline Ant I mom & $3.00 \mathrm{UJ}$ & $3.40 \mathrm{UJ}$ & $3.20 \mathrm{uJ}$ & $3.30 \mathrm{U}$ & $3.10 \mathrm{UJ}$ \\
\hline Arsente & $1.10 \mathrm{U}$ & $1.30 \mathrm{U}$ & $1.30 \mathrm{v}$ & $1.70 \mathrm{~V}$ & 2.30 \\
\hline Deriven & 93.40 & 87.70 & 82.50 & 57.60 & 60.30 \\
\hline iervillium & 0.65 & 0.22 & 0.49 & 0.30 & $0.13 \mathrm{~V}$ \\
\hline cedalum & $0.18 \mathrm{v}$ & $0.20 \mathrm{U}$ & $0.19 \mathrm{U}$ & $0.20 \mathrm{~V}$ & $0.18 \mathrm{~V}$ \\
\hline cololu & 5890.00 & 6340.00 & $\$ 310.00$ & 7930.00 & 8180.00 \\
\hline Chromilu & 6.10 & 5.20 & 6.60 & 4.90 & 3.50 \\
\hline Cobalt & 9.10 & 10.80 & 9.90 & 9.60 & 10.10 \\
\hline Coppor & 16.10 & 16.20 & 12.90 & 13.30 & 12.60 \\
\hline Iron & 18500.00 & 21100.00 & 18900.00 & 19400.00 & 19800.00 \\
\hline loed & 0.00 & 5.60 & 5.20 & 4.60 & $3.30 \mathrm{n}$ \\
\hline Mocrmelum & 4280.00 & 4310.00 & 4060.00 & 4110.00 & 4020.00 \\
\hline Menpanese & 260.00 & 268.00 & 262.00 & 266.00 & 269.00 \\
\hline Mareury & $0.04 v$ & $0.05 \mathrm{u}$ & $0.05 \mathrm{~V}$ & $0.05 \mathrm{U}$ & $0.05 v$ \\
\hline Niekol & 9.20 & $6.90 \mathrm{~B}$ & $7.50 \mathrm{~s}$ & 6.900 & $6.50 \mathrm{~B}$ \\
\hline Poteneslen & 1300.00 & $955.00 \mathrm{~B}$ & 903.00 & 1450.00 & 990.00 \\
\hline soleniun & $0.63 v$ & $0.68 v$ & $0.70 \mathrm{U}$ & $0.70 \mathrm{U}$ & $0.68 \mathrm{US}$ \\
\hline 811 ver & $0.45 v$ & $1.10 \mathrm{~V}$ & $0.62 \mathrm{v}$ & $0.83 \mathrm{v}$ & 0.81 \\
\hline sediun & $176.00 \mathrm{~J}$ & $192.00 \mathrm{~J}$ & $218.00 \mathrm{~J}$ & $228.00 \mathrm{~J}$ & $198.00 \mathrm{~J}$ \\
\hline Thatllum & $0.23 \mathrm{~V}$ & $0.24 \mathrm{~V}$ & $0.25 \mathrm{~V}$ & $0.29 \mathrm{v}$ & $0.60 \mathrm{UJ}$ \\
\hline Venediva & 40.40 & 48.30 & 67.20 & 43.60 & 32.20 \\
\hline zine & 67.90 & 45.70 & 39.10 & 40.80 & 43.30 \\
\hline Crentes & mn & Wh & NR & Nn & NR \\
\hline $68 \cdot 137$ & 0.36 & 0.34 & 0.43 & 0.31 & 0.071 \\
\hline $8 R \cdot 90$ & 0.42 & 0.11 & 0.096 & 0.26 & 0.36 \\
\hline
\end{tabular}

Note: All metale ore reported in mo/kg. Redlonuclides ore reported in pci/em.

- Analye found in seseclated blenk as well os semple.

$J$ - Mor deteeted, associated value is estimated.

Wh - Menreporteble.

- Date ore unuscble.

$U$ - Mot dotected, value reported is sumple quantitetion IImit.

UJ - Not detected, mar not eccurately reflect semple quantitation limit. 
Table F-4. Ant Mound Results from N Reactor in 1992.

\begin{tabular}{|c|c|c|c|c|c|}
\hline \multirow{3}{*}{ Const ituent } & \multicolumn{5}{|c|}{ Semple Identification Numbers and Locations } \\
\hline & $116-N-1$ & $116-N-1$ & $116-N-1$ & $116-N-1$ & Control \\
\hline & 807908 & $\begin{array}{c}807909^{\circ} \\
(807910)\end{array}$ & $\begin{array}{c}\text { Borg10 } \\
\text { Dupl icate }\end{array}$ & B07911 & 807918 \\
\hline Aluminum & 6530.00 & $\pi 770.00$ & 7380.00 & 7560.00 & 4560.00 \\
\hline Ant imony & $3.40 \mathrm{UJ}$ & $3.30 \mathrm{UJ}$ & $3.30 \mathrm{ud}$ & $3.20 \mathrm{UJ}$ & 3.10 UJ \\
\hline Arsenic & 3.20 & $1.60 \mathrm{~B}$ & 2.10 & 2.30 & 2.30 \\
\hline Bariun & 68.70 & 79.70 & 77.10 & 80.60 & 68.30 \\
\hline Beryllium & $0.34 \mathrm{U}$ & $0.13 u$ & $0.10 u$ & $0.10 u$ & $0.13 U$ \\
\hline Cachiun & $0.20 u$ & $0.20 \mathrm{U}$ & $0.20 \mathrm{U}$ & $0.19 \mathrm{U}$ & $0.18 \mathrm{U}$ \\
\hline calciun & 5730.00 & 4240.00 & 3630.00 & 4060.00 & 8180.00 \\
\hline Chromiun & 8.20 & 8.80 & 10.00 & 8.90 & 5.50 \\
\hline Cobalt & $9.50 \mathrm{~B}$ & 10.70 & $9.60 \mathrm{~B}$ & 9.008 & 10.10 \\
\hline Copper & 14.90 & 14.70 & 13.70 & 15.20 & 12.60 \\
\hline Iron & 19100.00 & 22600.00 & 18600.00 & 18600.00 & 19800.00 \\
\hline Leed & $4.00 \mathrm{R}$ & $3.50 \mathrm{R}$ & $4.80 R$ & $4.40 \mathrm{H}$ & $5.50 R$ \\
\hline Magnes iun & $42 k 0.00$ & 4240.00 & 3930.00 & 3990.00 & 4020.00 \\
\hline Menganese & 308.00 & 391.00 & 360.00 & 339.00 & 269.00 \\
\hline Mercury & $0.05 \mathrm{U}$ & $0.05 \mathrm{U}$ & $0.05 \mathrm{U}$ & $0.05 U$ & $0.05 U$ \\
\hline Nickel & 9.70 & 9.50 & 9.50 & 9.30 & $6.50 \mathrm{~B}$ \\
\hline Potassium & 1700.00 & 2260.00 & 2230.00 & 2440.00 & 990.00 \\
\hline Selenium & $0.70 \mathrm{UJ}$ & 0.77 Us & $0.71 \mathrm{UJ}$ & 0.77 UJ & $0.68 \mathrm{UJ}$ \\
\hline silver & $0.54 \mathrm{~B}$ & $1.00 \mathrm{~B}$ & $0.99 \mathrm{~B}$ & $0.65 \mathrm{~B}$ & $0.81 \mathrm{~B}$ \\
\hline Sodium & $219.00 \mathrm{~J}$ & $206.00 \mathrm{~J}$ & $198.00 \mathrm{~J}$ & $203.00 \mathrm{~J}$ & $198.00 \mathrm{~J}$ \\
\hline Thalliun & $0.68 \mathrm{U}$ & $0.75 \mathrm{U}$ & 0.69 UJ & $0.75 \mathrm{U}$ & $0.66 \mathrm{UJ}$ \\
\hline Venediun & 40.60 & 54.00 & 41.20 & 41.60 & 52.20 \\
\hline Zine & 45.80 & 53.50 & 45.40 & 49.90 & 43.30 \\
\hline \multicolumn{6}{|l|}{ Cyonide } \\
\hline Cs-137 & 0.19 & 0.52 & 0.52 & 0.14 & 0.07 \\
\hline sr -90 & 0.26 & -0.62 & -0.06 & 0.50 & 0.36 \\
\hline
\end{tabular}

NOTE: All metals are reported in mg/kg. Radionuclides are reported in pci/om.

$B$ a Anolyte found in associated blank as well as semple.

$J=$ Not detected; associated value is estimated.

NR = Nonreportable.

$R$ = Data are unusable.

$U$ = Mot detected; value reported is sample quantitation limit.

UJ = Not detected; may not accurately reflect sample quantitation limit.

Duplicate sample taken. 
Table F-5. Ant Mound Soll Results from BC Reactor in 1992.

\begin{tabular}{|c|c|c|c|c|c|c|}
\hline \multirow{3}{*}{ Const ituent } & \multicolumn{6}{|c|}{ Semple Identification Numbers and Locations } \\
\hline & $118-8-1$ & $118-C-4$ & $118-c-4$ & $118-C-1$ & $118-c-1$ & Control \\
\hline & B07820 & 807824 & 807825 & 807826 & Bo7827 & B07918 \\
\hline Aluminm & 8430.00 & 6210.00 & 5550.00 & 5790.00 & 5280.00 & 4560.00 \\
\hline Ant inony & $3.10 R$ & $3.40 \mathrm{R}$ & $3.10 R$ & $3.00 \mathrm{R}$ & $3.20 R$ & $3.10 \mathrm{UJ}$ \\
\hline Arsenic & 2.00 & 0.998 & $0.57 \mathrm{U}$ & $0.74 \mathrm{~B}$ & 0.79 & 2.30 \\
\hline Barium & 316.00 & 323.00 & 57.20 & 73.10 & 57.30 & 68.30 \\
\hline Beryllium & $0.54 \mathrm{U}$ & $0.66 \mathrm{U}$ & $0.64 \mathrm{U}$ & $0.52 U$ & $0.40 \mathrm{U}$ & $0.13 \mathrm{U}$ \\
\hline Cacmivn & $0.18 u$ & $0.20 u$ & $0.18 \mathrm{U}$ & $0.18 U$ & $0.19 u$ & $0.18 \mathrm{U}$ \\
\hline Calcium & 9630.00 & 6460.00 & 4220.00 & 4080.00 & 5020.00 & 8180.00 \\
\hline Chromiun & 7.20 & 4.80 & 6.30 & 6.90 & 13.20 & 5.50 \\
\hline Cobalt & $8.50 \mathrm{~B}$ & $9.80 \mathrm{~B}$ & 10.20 & 9.00 & 11.70 & 10.10 \\
\hline Copper & 19.60 & 16.50 & 17.80 & 15.70 & 17.20 & 12.60 \\
\hline Iron & 16100.00 & 18100.00 & 19400.00 & 17000.00 & 20800.00 & 19800.00 \\
\hline Lead & $4.50 \mathrm{~J}$ & $8.10 \mathrm{~J}$ & $9.90 \mathrm{~J}$ & $12.10 \mathrm{~J}$ & $8.30 \mathrm{~J}$ & $5.50 R$ \\
\hline Megnesiun & 4370.00 & 4060.00 & 4130.00 & 3740.00 & 4210.00 & 4020.00 \\
\hline Manganese & 263.00 & 298.00 & 280.00 & 289.00 & 300.00 & 269.00 \\
\hline Mercury & $0.04 \mathrm{U}$ & $0.05 \mathrm{U}$ & $0.05 \mathrm{U}$ & $0.04 \mathrm{U}$ & $0.04 \mathrm{U}$ & $0.05 \cup$ \\
\hline Nickel & 8.40 & $7.40 \mathrm{~B}$ & 9.30 & 7.60 & 13.30 & $6.50 \mathrm{~B}$ \\
\hline Potassiva & 1670.00 & 1540.00 & 1390.00 & 1710.00 & 1010.00 & 990.00 \\
\hline Seleniun & $0.69 \mathrm{UJ}$ & 0.73 us & 0.75 & 0.73 ud & 0.76 UJ & $0.68 \mathrm{UJ}$ \\
\hline silver & $0.46 \mathrm{U}$ & $0.50 \mathrm{U}$ & $1.30 \mathrm{U}$ & $0.87 U$ & $1.00 \mathrm{U}$ & $0.81 \mathrm{~B}$ \\
\hline Sodium & $467.00 \mathrm{~J}$ & $256.00 \mathrm{~J}$ & $193.00 \mathrm{~J}$ & $163.00 \mathrm{~J}$ & $212.00 \mathrm{~J}$ & $198.00 \mathrm{~J}$ \\
\hline Thallium & $0.69 \mathrm{U}$ & $0.73 u$ & $0.75 \mathrm{U}$ & $0.73 \mathrm{U}$ & $0.76 \mathrm{U}$ & $0.66 \mathrm{UJ}$ \\
\hline Vanedium & 40.80 & 37.40 & 43.10 & 38.30 & 51.80 & 52.20 \\
\hline zinc & 46.00 & 41.50 & 46.50 & 49.00 & 53.90 & 43.30 \\
\hline Cyanide & NR & NR & NR & NR & NR & NR \\
\hline Cs-137 & 0.15 & 0.21 & 0.45 & 0.24 & 0.37 & \\
\hline $5 r=90$ & 0.07 & 0.088 & -0.06 & -0.28 & -0.620 & \\
\hline Tc- 99 & & & & & & \\
\hline
\end{tabular}

NOTE: All metals are reported in mo/kg. Radionuclides are reported in $\mathrm{pCi} / \mathrm{gm}$.

$B$ - Analyte found in associated blank as well as sample.

$J=$ Not detected; associated value is estimated.

NR = Nonreportable.

$R=$ Data are unusable.

$U$ = Not detected; value reported is semple quantitation limit.

UJ = Not detected; may not accurately reflect sample quantitation limit. 
WHC-EP-0620

\section{APPENDIX G}

\section{ANALYTICAL RESULTS FOR SMALL MAMMAL BURROWS}


WHC-EP-0620

This page intentionally left blank. 
Table G-1. Burrow Mound Soll Results from F Reactor in 1992.

\begin{tabular}{|c|c|c|c|c|c|c|}
\hline \multirow{3}{*}{ Const i tuent } & \multicolumn{6}{|c|}{ semple Identifleation Numbers and Locations } \\
\hline & $126-F-1$ & $126-F-1$ & surf. Cont. & $116-f-1$ & $118 \cdot F \cdot 2$ & Control \\
\hline & 106N96 & B06N97 & $006 N 99$ & BOENBI & BO6NB3 & Bor920 \\
\hline Aluminum & 6220.00 & 6380.00 & 4960.00 & 5930.00 & 5590.00 & 7630.00 \\
\hline Ant imony & $3.40 \mathrm{R}$ & $3.10 R$ & $3.40 \mathrm{R}$ & $3.30 \mathrm{UJ}$ & $3.30 \mathrm{Ud}$ & 3.20 Ud \\
\hline Arsenle & $0.57 \mathrm{U}$ & 0.80 & 1.00 & 1.90 & $1.10 \mathrm{U}$ & 3.00 \\
\hline Barlun & 86.00 & 218.00 & 78.70 & 144.00 & 58.00 & 105.00 \\
\hline Berylllun & $0.39 \mathrm{U}$ & $0.54 \mathrm{U}$ & $0.39 \mathrm{U}$ & $0.49 \mathrm{~B}$ & $0.40 \mathrm{~B}$ & $0.13 \mathrm{U}$ \\
\hline Cadmiun & $0.20 \mathrm{U}$ & $0.18 \mathrm{U}$ & $0.20 \mathrm{U}$ & $0.20 \mathrm{U}$ & $0.19 \mathrm{U}$ & $0.19 \mathrm{U}$ \\
\hline calctun & 3710.00 & 6070.00 & 3970.00 & 5230.00 & 3300.00 & 11700.00 \\
\hline chromilum & 6.50 & 7.40 & 6.60 & 8.40 & 8.10 & 10.90 \\
\hline Cobalt & $8.90 \mathrm{~B}$ & 8.500 & $8.20 \mathrm{~B}$ & $7.80 \mathrm{I}$ & $9.10 \mathrm{~B}$ & 10.40 \\
\hline Copper & 10.40 & 12.40 & 10.20 & 11.40 & 9.40 & 11.60 \\
\hline Iron & 17100.00 & 16200.00 & 15200.00 & 16100.00 & 18900.00 & 20900.00 \\
\hline Lead & $3.50 \mathrm{~J}$ & $4.30 \mathrm{~J}$ & $8.50 \mathrm{~J}$ & 3.10 & 3.50 & $13.50 R$ \\
\hline Magnesium & 3800.00 & 4220.00 & 3900.00 & 3980.00 & 3800.00 & 4810.00 \\
\hline Manganose & 284.00 & 287.00 & 241.00 & 235.00 & 272.00 & 388.00 \\
\hline Mercury & $0.05 U$ & $0.05 \mathrm{U}$ & $0.05 U$ & $0.05 \mathrm{U}$ & $0.04 \mathrm{U}$ & $0.05 \mathrm{U}$ \\
\hline Nickel & 8.60 & 8.10 & 9.10 & $7.70 \mathrm{~s}$ & 7.90 & 9.70 \\
\hline Potasesiun & 1350.00 & 1560.00 & 1310.00 & 1030.00 & 1600.00 & 1870.00 \\
\hline selentum & $0.75 \mathrm{U}$ & 0.68 us & $0.72 \mathrm{uJ}$ & $0.67 \mathrm{U}$ & $0.69 \mathrm{U}$ & $0.77 \mathrm{us}$ \\
\hline sllver & $0.78 \cup$ & $0.61 \mathrm{U}$ & $0.60 \mathrm{U}$ & $0.87 \mathrm{U}$ & $1.10 \mathrm{U}$ & 0.87 \\
\hline sodivn & $184.00 \mathrm{~J}$ & $279.00 \mathrm{~J}$ & $182.00 \mathrm{~J}$ & $264.00 \mathrm{~J}$ & $142.00 \mathrm{~J}$ & $208.00 \mathrm{~J}$ \\
\hline Thalliven & $0.73 \mathrm{U}$ & $0.68 U$ & $0.72 \mathrm{U}$ & $0.24 \mathrm{U}$ & $0.25 U$ & $0.73 \mathrm{U}$ \\
\hline Vanedium & 42.80 & 41.40 & 39.50 & 44.00 & 52.10 & 32.10 \\
\hline Zinc & 36.40 & 36.00 & 36.30 & 34.20 & 38.60 & 115.00 \\
\hline Cyanids & MR & MR & NR & NR & MR & NR \\
\hline$C 8-137$ & $u$ & 0.08 & 0.17 & 0.34 & $u$ & U \\
\hline $8 r-90$ & -0.027 & 0.200 & 0.063 & 0.220 & 0.00 & 0.091 \\
\hline
\end{tabular}

NOTE: All metals are reported in $\mathrm{mg} / \mathrm{kg}$. Redionuclides are reported in $\mathrm{pcl} / \mathrm{om}$.

B - Analyte found In associated blank as woll as sample.

$J$ - Not detected; assoclated value is estimated.

NR = Nonreportable.

R = Data are unusable.

$U$ - Not detected; value reported is semple quantitation IImit.

UJ = Not detected; may not accurately reflect semple quantitation limit. 
Table G-2. Burrow Results from H Reactor Collected in 1992.

\begin{tabular}{|c|c|c|c|c|}
\hline \multirow{3}{*}{ Const I tuant } & \multicolumn{4}{|c|}{ supple Identifleation Murbars and Locations } \\
\hline & $118 \cdot N \cdot 3$ & $118 \cdot N \cdot 3$ & $118-H \cdot 7$ & Control \\
\hline & $\cos n e 6$ & 806NB7 & cosnco & 107920 \\
\hline Aluminn & 7320.00 & 9790.00 & 4990.00 & 7630.00 \\
\hline Ane I mony & 3.00 Ud & 3.40 Us & $3.30 \mathrm{UJ}$ & $3.20 \mathrm{UJ}$ \\
\hline Arsenic & 3.10 & 4.90 & 3.30 & 3.00 \\
\hline Barlum & 102.00 & 103.00 & 46.90 & 105.00 \\
\hline Beryllium & 0.59 & 0.39 & 0.37 & $0.13 \mathrm{U}$ \\
\hline Cecinlun & $0.18 \mathrm{u}$ & $0.20 \mathrm{U}$ & $0.19 \mathrm{U}$ & $0.19 \mathrm{U}$ \\
\hline catclun & 4690.00 & 3430.00 & 5340.00 & 11700.00 \\
\hline Chromiven & 8.90 & 12.20 & 7.70 & 10.90 \\
\hline cobult & 6.80 & $9.90 \mathrm{~B}$ & $6.60 \mathrm{~B}$ & 10.40 \\
\hline Copper & 14.20 & 12.80 & 11.10 & 11.60 \\
\hline Iron & 15800.00 & 20600.00 & 14200.00 & 20900.00 \\
\hline Leed & 17.00 & 14.90 & 5.90 & $13.50 \mathrm{R}$ \\
\hline Maenesilu & 4200.00 & 4710.00 & 3790.00 & 4890.00 \\
\hline Manganose & 316.00 & 370.00 & 223.00 & 384.00 \\
\hline Morcury & $0.05 \cup$ & $0.05 U$ & $0.06 \mathrm{U}$ & $0.05 U$ \\
\hline Nickel & 8.90 & 12.00 & 8.30 & 9.70 \\
\hline Potessiven & 2340.00 & 2480.00 & $771.00 \mathrm{~B}$ & 1870.00 \\
\hline selentun & 0.70 Us & $0.68 \mathrm{uJ}$ & $0.70 \mathrm{U}$ & $0 . \pi \mathrm{UJ}$ \\
\hline silver & $0.58 \mathrm{U}$ & $1.10 \mathrm{U}$ & $0.48 \mathrm{U}$ & 0.87 \\
\hline sodiun & $188.00 \mathrm{~J}$ & $157.00 \mathrm{~J}$ & $119.00 \mathrm{~J}$ & $208.00 \mathrm{~J}$ \\
\hline Thalliven & $0.25 \mathrm{uJ}$ & $0.25 U$ & $0.25 \cup$ & $0.73 \mathrm{U}$ \\
\hline Venodium & 32.20 & 44.00 & 39.90 & 32.10 \\
\hline Zine & 49.60 & 46.00 & 29.80 & 115.00 \\
\hline Crenles & NR & MR & NR & NR \\
\hline$c 8 \cdot 137$ & 0.50 & $\mathbf{u}$ & $\mathbf{u}$ & u \\
\hline $5 R-90$ & $\cdot 0.39$ & 0.079 & 0.31 & 0.091 \\
\hline
\end{tabular}

NOTE: All matals are reported in mo/kg. Radionucllides are reported in pcl/an.

\footnotetext{
a Anslyte found in seseciated blenk as wall as semple.

$J$ - Not detected; associated value is estimated.

MR - Nonrenortable.

R - Data ore unuseble.

$U$ - Not detected; value reported le semple quantitetion limit.

UJ = Not detected; may not accurately reflect semple quentitation limit.
} 
Table G-3. Burrow Results from D Reactor Collected in 1992.

\begin{tabular}{|c|c|c|c|c|c|}
\hline \multirow{3}{*}{ Constltuent } & \multicolumn{5}{|c|}{ 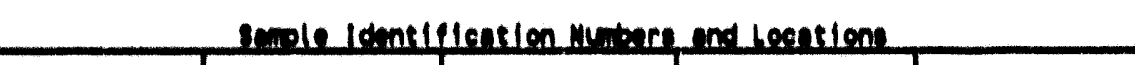 } \\
\hline & $116 \cdot 01 . \cdot 9$ & $116 \cdot 0 \cdot 3$ & $116 \cdot 0 \cdot 1$ & $116 \cdot 0 \cdot 2$ & Gentrol \\
\hline & sosucs & nosucs & nosuct & $\operatorname{los} 459$ & norazo \\
\hline Alvainn & 5840.00 & 690.00 & 969.09. & 5110.00 & 2830.00 \\
\hline Antiman & 3.6044 & $3.20 \times 14$ & 3.00014 & 3.204 & 3.20 .4 \\
\hline Aresnle & 2,20 & $3.10+$ & 2.50 & $1.40 \mathrm{v}$ & 3.00 \\
\hline Ancin & 100.00 & 103.00 & 81.30 & 36.9 & 105.00 \\
\hline eryll1u & 0.620 & 0.391 & 0.31 & 0.600 & $0.13 u$ \\
\hline cectalu. & $0.20 \mathrm{u}$ & $0.19 \mathrm{y}$ & 0.184 & 0.194 & -0.194 \\
\hline crlatur. & 11700.00 & 9010.00 & .480 .00 & -4190.00 & 11709.09 \\
\hline chrealum & 2.70 & 2.30 & 2.10 & 8,00 & 10.90 \\
\hline cobalt & 1.901 & 2.201 & 10,00 & 8.60 & 10.40 \\
\hline Gooner & 16.90 & 13.20 & 13.40 & 10.90 & 11.60 \\
\hline Iren & 17600.00 & 19200.00 & 20100.00 & 17100.00 & 30000,00 \\
\hline lend & 4.90 & 4.60 & 1.20 & 7.00 & $13.50 \mathrm{R}$ \\
\hline Marneelun & 4680.00 & 4980.00 & 6370.00 & 3980.00 & 4810.00 \\
\hline Menremese & 27.00 & 319.00 & $2 \%, 00$ & 235.00 & $38 ., 00$ \\
\hline Meraury & 0.0 .4 & $0.06 \mathrm{y}$ & $0.02 \mathrm{H}$ & $0.05 \mathrm{~L}$ & 0.050 \\
\hline Mickel & 8.60 & 10.50 & 8.30 & 2.20 & 9.70 \\
\hline Potensin & 1370.00 & 1610.00 & 12310.00 & 1400.00 & 1870.00 \\
\hline selentur & 0.62 .44 & $0.6 \times 4$ & 0.634 & 0.44 & $0.7 \pi 4$ \\
\hline sllver & $0.7 \mathrm{u}$ & $0.76 \mathrm{U}$ & $0.82 \mathrm{U}$ & $0.80 \mathrm{U}$ & 0.071 \\
\hline sodivn & $167.00 \mathrm{~d}$ & $156.00 \mathrm{~d}$ & $176.00 \mathrm{~d}$ & $200.00 \mathrm{~d}$ & $20.00 \mathrm{~d}$ \\
\hline Thalliven & $0.25 u$ & $0.26 \mathrm{UJ}$ & $0.23 u$ & $0.23 u$ & $0.7 u$ \\
\hline venodiun & 39.90 & 49.60 & 30.80 & 43.60 & 32.10 \\
\hline $2 \ln c$ & 10.70 & 40.50 & $46+50$ & 36,30 & 115.00 \\
\hline Cyenide & Nh & NR & MR & MR & MR \\
\hline$c s \cdot 137$ & 0.17 & $y$ & 0.16 & 0.16 & 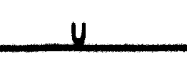 \\
\hline $5 R-90$ & 0.055 & 0.18 & 0.13 & 0.077 & 0.091 \\
\hline
\end{tabular}

NOTE: All metals are reported in ma/kg. Redionuclides are reported in $\mathrm{pCl} / \mathrm{om}$.

- Analyte found in assoclated blank as woll us semple.

$J$ - Mot dotectedi asesoclated value in estimated.

NR - Nonreportable.

- Dote ore unuseble.

$U$ - Not datected; volus reported is sumple quantitation IImit.

UJ = Not dotected; may not accurately reflect smple quantitation 1 imlt. 
Table G-4. Burrow Mound Soll Results from 1992 at $K$ Reactor. (sheet 1 of 2)

\begin{tabular}{|c|c|c|c|c|}
\hline \multirow{3}{*}{ Conseltuent } & \multicolumn{4}{|c|}{ 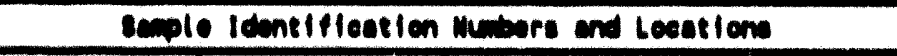 } \\
\hline & $118-k \cdot 1$ & $110 \cdot k \cdot 1$ & $110 \cdot k \cdot 1$ & $116 \cdot k-1$ \\
\hline & 00728 & 007900 & 007801 & 007002 \\
\hline Aluinen & 6140.00 & 5220.00 & $\$ 420.00$ & 3070.00 \\
\hline Anetering & $3.30 \mathrm{n}$ & $3.40 h$ & 3.201 & $3.20 \mathrm{n}$ \\
\hline Arsenle & 2.40 & 1.000 & 2.10 & 0.670 \\
\hline Darluen & 37.30 & 36.40 & 98.00 & 43.70 \\
\hline Doryllium & $0.35 \cup$ & $0.26 \mathrm{U}$ & $0.35 \mathrm{U}$ & $0.20 \mathrm{~V}$ \\
\hline cocinlun & $0.19 \mathrm{~V}$ & $0.20 \mathrm{U}$ & $0.19 \mathrm{~V}$ & $0.19 \mathrm{U}$ \\
\hline colelu & 460.00 & 6110.00 & 780.00 & 5210.00 \\
\hline Chrealiu & 8.00 & 6.70 & 6.30 & 3.60 \\
\hline Cabole & 7.300 & 6.001 & 7.500 & 11.00 \\
\hline Cogeper & 13.30 & 11.30 & 11.20 & 16.00 \\
\hline Iren & 14000.00 & 13400.00 & 16600.00 & 18700.00 \\
\hline Lead & $0.90 \mathrm{~J}$ & $7.60 \mathrm{~d}$ & $6.00 \mathrm{~J}$ & $3.30 \mathrm{~d}$ \\
\hline Mocnestive & 6720.00 & 6190.00 & 4490.00 & 3790.00 \\
\hline Menemenes & 261.00 & 244.00 & 26.00 & 201.00 \\
\hline Moroury & $0.04 \mathrm{~V}$ & $0.05 \mathrm{U}$ & $0.04 \mathrm{U}$ & $0.05 \mathrm{U}$ \\
\hline Nlokel & 9.00 & 0.40 & 7.70 & 7.100 \\
\hline Poteses iv & 1620.00 & 1670.00 & 1630.00 & 70.000 \\
\hline soleniu & $0.0 v$ & $0.73 U$ & $0.76 \mathrm{WJ}$ & $0.71 \mathrm{v}$ \\
\hline sllver & $0.60 \mathrm{~V}$ & $0.50 \mathrm{U}$ & $0.50 \mathrm{U}$ & $0.7 \mathrm{U}$ \\
\hline sodiu & $135.00 \mathrm{~J}$ & $130.00 \mathrm{~J}$ & $160.00 \mathrm{~J}$ & $213.00 \mathrm{~J}$ \\
\hline Tholliv & $0.70 \mathrm{U}$ & $0.7 \mathrm{U}$ & $0.74 \mathrm{v}$ & $0.71 \mathrm{U}$ \\
\hline Venediun & 31.10 & 26.90 & 30.90 & 60.90 \\
\hline Zine & $\$ 10.00$ & 48.00 & 57.40 & 217.00 \\
\hline Cyenide & Mn & Mn & Mn & Mn \\
\hline Co.137 & 0.92 & 0.65 & 0.61 & $u$ \\
\hline $8 r \cdot 90$ & -0.20 & -0.56 & -0.7 & 0.021 \\
\hline Te- 9 & & & & \\
\hline
\end{tabular}
pol/cim.

Morte All motals ore reported in my/kg. Redlonvellase are reported in

- Anslyte fand In esesceloted blank as will as semple.

J Not dotected ascociated volus lo estiminted.

MR - Nonreporteble.

R. Dote are unuseble.

$U$ - Not detected volus reported is scople auntitetion IImit.

UJ a Not dotecteds may not eccurately rofleet semple quentitation linite.

Dupl leote semple taken. 
Table G-4. Burrow Mound Soll Results from K Reactor In 1992. (sheet 2 of 2)

\begin{tabular}{|c|c|c|c|c|}
\hline \multirow{3}{*}{ Constituent } & \multicolumn{4}{|c|}{ 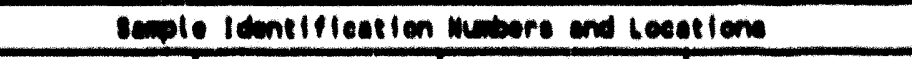 } \\
\hline & $116 \cdot x \cdot 1$ & $116 \cdot x \cdot 1$ & $110-x-1$ & Centrol \\
\hline & soreas & corros" & $\begin{array}{l}\text { convos } \\
\text { Duplleste }\end{array}$ & $\operatorname{cor} 20$ \\
\hline Aluminn & 9810.00 & 7030.00 & 3800.00 & 7010.00 \\
\hline Ane lemany & 3.301 & $3.30 n$ & $3.10 \mathrm{~W}$ & $3.20 \mathrm{UJ}$ \\
\hline Arsenle & 0.931 & 1.300 & 0.001 & 3.00 \\
\hline Derin & 6.00 & 7.09 & 61.00 & 108.00 \\
\hline Cervllius & $0.67 \mathrm{~V}$ & $0.36 \mathrm{U}$ & 0.100 & $0.13 \mathrm{~V}$ \\
\hline coctivin & $0.20 \mathrm{~V}$ & $0.10 \mathrm{U}$ & $0.11 \mathrm{v}$ & $0.10 \mathrm{~V}$ \\
\hline colalun & 310.00 & 3760.00 & 470.00 & 11700.00 \\
\hline chrealu & 7.60 & 7.60 & 3.10 & 10.60 \\
\hline Cecolle & 9.300 & 0.90 & 9.30 & 10.60 \\
\hline conper & 16.70 & 15.50 & 16.00 & 11.60 \\
\hline Iren & 16000.00 & 1000.00 & 1000.00 & 20000.00 \\
\hline Lead & $6.00 \mathrm{~J}$ & $6.60 \mathrm{~J}$ & 9.70 & $13.90 n$ \\
\hline mecneslum & 380.00 & 3070.00 & 3710.00 & 4010.00 \\
\hline Mancenese & 390.00 & 33.00 & $281.00 \mathrm{~J}$ & 39.00 \\
\hline Mreury & $0.05 \mathrm{U}$ & $0.05 \mathrm{U}$ & $0.05 \mathrm{v}$ & $0.05 \mathrm{U}$ \\
\hline Niakol & 10.10 & 9.90 & 9.000 & 9.70 \\
\hline Potecestun & 1900.00 & 290.00 & 00.000 & 1870.00 \\
\hline selonive & 0.74 & $0.7 \mathrm{w}$ & $0.69 \mathrm{~V}$ & $0.77 \mathrm{u}$ \\
\hline 811 ver & $0.7 v$ & $0.76 \mathrm{v}$ & $0.12 \mathrm{U}$ & 0.070 \\
\hline cedilu & $161.00 \mathrm{~J}$ & $170.00 \mathrm{~d}$ & $207.00 \mathrm{~d}$ & $209.00 \mathrm{~J}$ \\
\hline Thalllu & 0.76 & $0.5 \cup$ & $0.30 \mathrm{U}$ & $0.7 \mathrm{~V}$ \\
\hline Vencalum & 37.30 & 30.00 & $0.51 \mathrm{n}$ & 52.10 \\
\hline $\operatorname{lin}$ & $n .7$ & 67.00 & $213.00 \mathrm{~J}$ & 113.00 \\
\hline Griside & min & $M$ & min & $m$ \\
\hline $60 \cdot 137$ & 0.16 & 0.31 & & $u$ \\
\hline$p+\infty$ & .0 .039 & .0 .73 & & 0.091 \\
\hline$i e \cdot 9$ & & & & \\
\hline
\end{tabular}
a110.

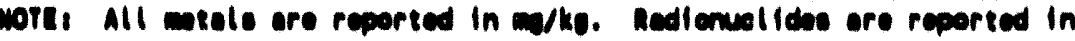

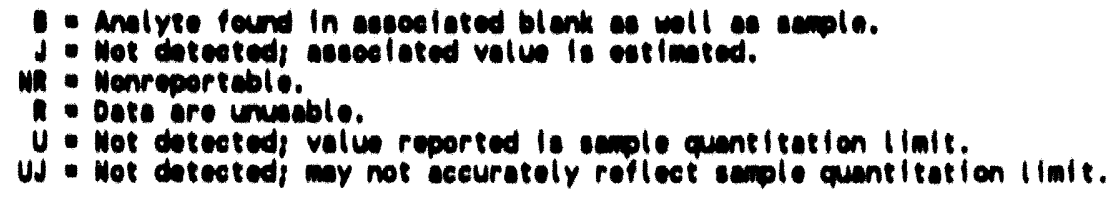


Table Q-5. Burrow Results from N Reactor in 1992.

\begin{tabular}{|c|c|c|c|c|c|}
\hline \multirow{3}{*}{ censel livent } & \multicolumn{5}{|c|}{ 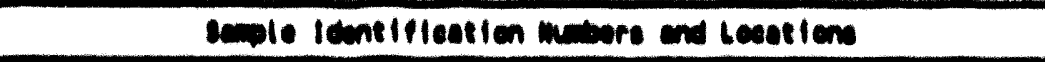 } \\
\hline & $116 \cdot 11 \cdot 2$ & $116 \cdot n \cdot 3$ & $116-n-1$ & $110 \cdot 1 \cdot 1$ & Centrol \\
\hline & corves & conves & corvor & corviz & corvas \\
\hline Alvinn & 7110.00 & 000.00 & 0000.00 & 000.00 & 700.00 \\
\hline imilnay & $3.00 \mathrm{Ud}$ & 3.30 us & $3.20 \mathrm{~J}$ & $3.30 \mathrm{wd}$ & $3.20 \mathrm{WJ}$ \\
\hline Areente & 1.000 & 2.0 & 2.20 & 2.30 & 3.00 \\
\hline Dorive & 9.40 & 0.70 & 6.60 & 6.60 & 103.60 \\
\hline cervilitum & $0.41 \mathrm{U}$ & $0.21 \mathrm{U}$ & $0.11 v$ & $0.10 \mathrm{U}$ & $0.13 \mathrm{U}$ \\
\hline cencina & $0.10 \mathrm{~V}$ & $0.20 \mathrm{~V}$ & $0.10 \mathrm{U}$ & $0.20 \mathrm{v}$ & $0.10 \mathrm{~V}$ \\
\hline cololun & 5200.00 & 300.00 & 340.00 & 370.00 & 11700.00 \\
\hline Chrealu & 10.70 & 11.90 & 12.10 & 11.30 & 10.90 \\
\hline cemole & 8.100 & 0.300 & 0.600 & 0.200 & 10.40 \\
\hline Cepaper & 14.10 & 19.70 & 16.10 & 13.60 & 11.60 \\
\hline Iren & 10200.00 & 10300.00 & 1000.00 & 10900.00 & 20000.00 \\
\hline lend & $9.20 \mathrm{n}$ & $4.60 n$ & $9.10 n$ & $5.90 \mathrm{n}$ & $13.90 \mathrm{a}$ \\
\hline nenerealu & 4220.00 & 400.60 & 400.00 & 4300.09 & 410.00 \\
\hline nervenese & 20.00 & 28.00 & 397.00 & 39.00 & 30.60 \\
\hline Mreum & 0.050 & $0.05 \mathrm{~V}$ & $0.05 \mathrm{v}$ & $0.00 \mathrm{v}$ & $0.05 v$ \\
\hline NIakal & 10.60 & 11.40 & 11.50 & 11.00 & $9 . \%$ \\
\hline Potcestiv & 1670.00 & 170.00 & 2010.00 & 2270.00 & 1070.00 \\
\hline colenim & 3.50 w & $0.71 \mathrm{u}$ & 0.7 us & $0.70 \mathrm{w}$ & $0 . n$ us \\
\hline ollver & 1.200 & 1.200 & 0.000 & 1.100 & 0.970 \\
\hline sedive & $103.00 \mathrm{~J}$ & $105.60 \mathrm{~J}$ & $108.00 \mathrm{~J}$ & $207.00 \mathrm{~J}$ & $205.00 \mathrm{~J}$ \\
\hline Thalllen & $0.69 \mathrm{us}$ & $0.49 \mathrm{WJ}$ & $0.7 \mathrm{~V}$ & $0.76 \mathrm{ud}$ & $0.7 \mathrm{u}$ \\
\hline Vanallum & 30.60 & 37.10 & 42.50 & 41.60 & 92.10 \\
\hline $2 \ln n$ & 63.20 & 30.60 & 13.00 & 97.20 & 119.00 \\
\hline Gyonlate & $m$ & $m$ & nin & $m$ & un \\
\hline $68 \cdot 137$ & $\mathbf{u}$ & 0.23 & $0.6 n$ & 0.66 & $\mathbf{v}$ \\
\hline $8 r \cdot c 0$ & 0.30 & 1.40 & 0.66 & 0.33 & 0.00 \\
\hline
\end{tabular}

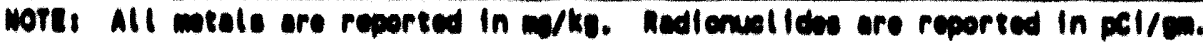

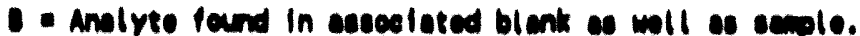

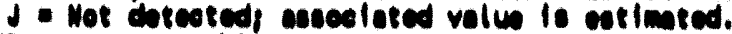

in - Nenreporteble.

- Oete ore vingble.

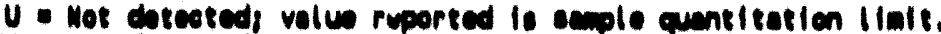

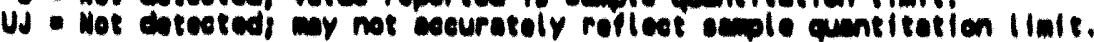


WHC-EP-0620

Table G-6. Burrow Mound So11 Results from BC Reactor

in 1992.

\begin{tabular}{|c|c|c|c|c|c|}
\hline \multirow{3}{*}{ centlituent } & \multicolumn{5}{|c|}{ 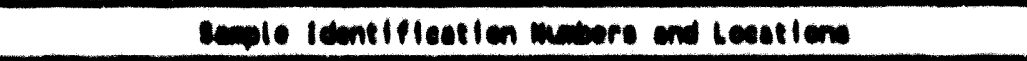 } \\
\hline & $110 \cdot 0 \cdot 1$ & $118 \cdot 1 \cdot 1$ & $110 \cdot 0 \cdot 1$ & $116 \cdot 6 \cdot 9$ & centrel \\
\hline & Corke & onne & coras & conth & $00 \mathrm{nan}$ \\
\hline Alinim & 1090.69 & 1600.60 & 090.00 & 710.00 & 700.00 \\
\hline Amingm & $3.30 \mathrm{n}$ & $3.40 \mathrm{n}$ & $3.20 \cap$ & $3.60 \mathrm{n}$ & 1.20 us \\
\hline Areanle & 2.0 & 3.90 & 1.200 & 1.900 & 3.60 \\
\hline entun & $10 \% .60$ & 1700.00 & 4.7 & 7.10 & 108.00 \\
\hline cervillien & 1.90 & 1.00 & $0.25 \cup$ & $0.15 \mathrm{U}$ & $0.15 \cup$ \\
\hline contun & $0.20 \cup$ & $0.20 \mathrm{~V}$ & $0.10 \mathrm{~V}$ & $0.20 \mathrm{~V}$ & $0.10 \mathrm{u}$ \\
\hline coletu & $m$ & 38000.00 & 300,00 & 490.00 & 11700.00 \\
\hline enrevilu & 0.03 & 0.10 & 7.20 & 11.20 & 10.60 \\
\hline secule & 0.600 & 12.50 & 0.600 & 0.000 & 10.40 \\
\hline ceppipr & 26.30 & 30.20 & 10.60 & 16.10 & 11.60 \\
\hline Iren & 11900.60 & 20490.00 & 1400.00 & 1000.00 & 20100.00 \\
\hline lead & $5.10 \mathrm{~d}$ & $0.60 \mathrm{~d}$ & $4.60 \mathrm{~J}$ & $4.00 \mathrm{~J}$ & $13.50 \mathrm{n}$ \\
\hline noreature & $0 \% .00$ & 600.60 & 390.00 & 440.00 & 410.00 \\
\hline Manumese & 20.00 & 310.00 & 20,00 & 27.00 & 39.00 \\
\hline meroury & 0.000 & $0.05 \cup$ & $0.04 \mathrm{~V}$ & $0.05 \cup$ & $0.05 \cup$ \\
\hline Wiekel & 14.20 & 17.00 & 0.30 & 12.90 & 0.70 \\
\hline Poteselve & 2140.60 & 1480.00 & 1600.60 & 1670.00 & 1070.00 \\
\hline colentur & $3.60 \mathrm{~V}$ & $3.60 \mathrm{v}$ & 0.74 us & 0.70 us & $0 . r$ us \\
\hline sliver & $0.7 \mathrm{v}$ & 0.50 U & 0.40 & $0.71 \cup$ & 0.070 \\
\hline sediu & $1840.00 \mathrm{~J}$ & $2000.00 \mathrm{~J}$ & $149.00 \mathrm{~J}$ & $204.00 \mathrm{~d}$ & $200.00 \mathrm{~J}$ \\
\hline inollin & $0.72 \mathrm{U}$ & $0.7 \mathrm{~V}$ & $0.76 \mathrm{~V}$ & $0.70 \mathrm{U}$ & $0.7 \cup$ \\
\hline venediun & 34.10 & 03.00 & 33.60 & 34.60 & 52.10 \\
\hline $21 n$ & 27.60 & 3.20 & 33.00 & 30.60 & 115.00 \\
\hline Grentes & $m$ & $m$ & m & in & min \\
\hline $60 \cdot 137$ & 0.22 & 0.20 & $\mathbf{u}$ & 0.18 & $\mathbf{u}$ \\
\hline spen & 0.12 & .0 .36 & 0.00 & 0.20 & 0.00 \\
\hline 1e.6 & & & & & \\
\hline
\end{tabular}

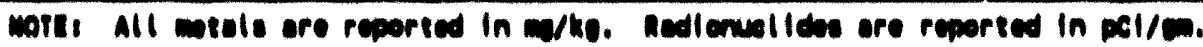

- Anolve fand In eseceloted blenk es wll en exple.

- Hot dotested sused loted volu to estiented.

- Menregertede.

- Dote ore unueble.

$U$ - Not dotcered volus reported lo ecople aumelestion II alt.

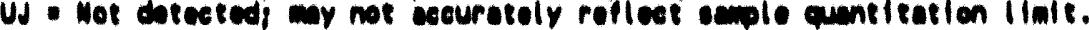


WHC - EP-0620

\section{APPENDIX H}

\section{RADIONUCLIDE VALUES IN SOIL ANO VEGETATION FROM 100 AREAS COLLECTED AS PART OF WESTINGHOUSE HANFORD COMPANY'S ENVIRONMENTAL MONITORING PROGRAM}


WHC-EP-0620

This page intentionally left blank. 
Table $\mathrm{H}-1$. Average Radionuclide Concentrations $(\mathrm{pC} / \mathrm{g})$ Detected in Soll Samples near the 1301-N Liquid Waste Disposal Facllity from 1980 through 1991.

\begin{tabular}{|c|c|c|c|c|c|}
\hline rour & ${ }^{4} \mathrm{Co}$ & $9 r$ & "I'co & $=19 u$ & in.t.tev \\
\hline 1840 & $1.31+01$ & $3.5 \div .01$ & $4.1 \div+00$ & mn & $2.3 \times 1.02$ \\
\hline Iest & $4.0 \pm+00$ & $7.0 \mathrm{e} \cdot 01$ & $0.11+00$ & in & 4.41 .02 \\
\hline 194 & $0.31+00$ & $2.7 \times .01$ & $2.71+00$ & $m$ & 1.01 .02 \\
\hline 1963 & 3.41000 & 1.31000 & $3.8 \div+00$ & $m$ & $4.31-02$ \\
\hline 194 & 2.011400 & $2.18 \cdot 01$ & $1.1 E+00$ & wn & $1.7 \pm .02$ \\
\hline 1905 & $1.31+01$ & 0.51 .01 & $3.91+00$ & $m$ & 3.28 .02 \\
\hline iens & $4.31+\infty 0$ & $2.2 \times .01$ & 2.511000 & $m n$ & $1.7 \times .02$ \\
\hline 1947 & 3.10400 & 3.41 .01 & $1.61+00$ & $3.4:-03$ & $2.2 \div .02$ \\
\hline 1008 & 7.88000 & $3.5 \cdot 1 \cdot 01$ & 2.01400 & $2.38 \cdot 03$ & $1.7 \mathrm{~m} \cdot 02$ \\
\hline 189 & $2.3+400$ & 1.51 .01 & $3.0 \times .01$ & 6.41 .03 & $4.0 \times \cdot 02$ \\
\hline 1900 & 4.71400 & $3.2 \times .01$ & $1.78+00$ & 4.61 .03 & $3.5 \times \cdot .02$ \\
\hline 1991 & 1.38401 & $2.7 \times .01$ & $1.3+\infty 00$ & $7.7=0.03$ & $3.3 \simeq .02$ \\
\hline
\end{tabular}

nim - Not reported.

Table $\mathrm{H}-2$. Average Radionuclide Concentrations ( $\mathrm{pCt} / \mathrm{g})$ Detected in $100 \mathrm{~N}$ Area Surface Soll Samples from 1980 through 1991.

\begin{tabular}{|c|c|c|c|c|c|}
\hline rear & $\omega_{60}$ & $9 r$ & "II'ce & 100 & 10,00404 \\
\hline 1900 & $0.5-01$ & $1.0 \mathrm{c} \cdot 01$ & $9.0 \div \cdot 01$ & un & 1.01 .02 \\
\hline 1901 & $1.3 \approx+\infty 0$ & 2.10 .01 & $1.0 \times+00$ & mn & $1.1 \times .02$ \\
\hline 192 & $1.61+00$ & $0.9 \times 02$ & 3.61 .01 & Mn & $5.0 \times \cdot 03$ \\
\hline 1993 & $2.7 \pm 100$ & $2.9-01$ & 4.61 .01 & mo & $0.5 \cdot .03$ \\
\hline 194 & $0.81-01$ & $2.8 \div .01$ & 0.21 .01 & $m$ & $1.6=.02$ \\
\hline 1908 & 1.21000 & $1.31 \cdot 01$ & $3.2 \cdot-01$ & Mn & $1.3: .02$ \\
\hline $19 e$ & $4.1 \cdot 01$ & $0.3 \times .02$ & $3.0 \div .01$ & $n n$ & $0.2 \times .03$ \\
\hline 1997 & $4.1+0.01$ & $1.1 \cdot 01$ & 3.01 .01 & $1.1+.03$ & $6.7 \times .03$ \\
\hline 1903 & $3.6 \times .01$ & 1.61 .01 & $3.9:-01$ & $4.5 \times \cdot 04$ & $9.5 \in-03$ \\
\hline 1900 & $1.6 \times-01$ & 2.18 .01 & $1.38 \cdot 01$ & $1.1 \div .03$ & $1.3 \times \cdot 02$ \\
\hline 1900 & 3.08 .01 & 1.21 .01 & $6.4=01$ & $0.7=0.06$ & $1.0+\cdot 02$ \\
\hline 1991 & $4.3 \pi \cdot 0 ?$ & 1.18 .01 & $4.5 \mathrm{t} \cdot 01$ & 6.28 .04 & $7.8 \cdot .03$ \\
\hline
\end{tabular}

in - Not reparted. 
Table $\mathrm{H}-3$. Average Radionuclide Concentrations ( $\mathrm{pCi} / \mathrm{g}$ ) Detected in 100-B/C Area Surface Soil Samples from 1981 to 1991.

\begin{tabular}{|c|c|c|c|c|c|}
\hline Year & ${ }^{60} \mathrm{CO}$ & ${ }^{90} \mathrm{Sr}$ & ${ }^{137} \mathrm{Cs}$ & ${ }^{238} \mathrm{Pu}$ & $239,240 \mathrm{Pu}$ \\
\hline 1981 & $5.7 E-01$ & NR & $1.2 E+00$ & NR & NR \\
\hline 1982 & $8.2 E-01$ & NR & $1.3 E+00$ & NR & NR \\
\hline 1983 & $4.2 \quad E-01$ & NR & $1.5 E+00$ & NR & NR \\
\hline 1984 & $5.4 E-01$ & $3.2 E-01$ & $1.9 E+00$ & $1.0 \mathrm{E}-03$ & $2.4 \quad E-02$ \\
\hline 1985 & $2.7 E-01$ & $2.4 E-02$ & $4.5 E-01$ & $2.9 \quad E-04$ & $8.8 \mathrm{E}-04$ \\
\hline 1986 & $1.8 E-01$ & $1.2 E-01$ & $6.4 E-01$ & $5.5 E-04$ & $8.3 E-03$ \\
\hline 1987 & $2.6 \mathrm{E}-01$ & $1.1 E-01$ & $9.2 E-01$ & $6.2 E-04$ & $1.4 \mathrm{E}-02$ \\
\hline 1988 & $2.7 \mathrm{E}-01$ & $3.9 E-01$ & $9.5 E-01$ & $6.2 \mathrm{E}-04$ & 3.0 E-02 \\
\hline 1989 & $2.6 \mathrm{E}-01$ & $3.5 E-01$ & $7.6 \mathrm{E}-01$ & 6.2 E-04 & $5.5 E-02$ \\
\hline 1990 & $1.1 E-01$ & $1.7 E-01$ & $7.4 E-01$ & $6.2 \mathrm{E}-04$ & $2.9 E-02$ \\
\hline 1991 & $6.4 E-01$ & $1.6 E-01$ & $9.0 E-01$ & $9.0 \quad E-04$ & $1.7 E-02$ \\
\hline
\end{tabular}

NR $=$ Not Reported.

Table $\mathrm{H}-4$. Average Radionuclide Concentrations ( $\mathrm{pC} / \mathrm{g}$ ) Detected in 100-D/DR Area Surface Soil Samples from 1981 to 1991.

\begin{tabular}{|c|c|c|c|c|c|}
\hline Year & ${ }^{60} \mathrm{Co}$ & ${ }^{90} \mathrm{Sr}$ & ${ }^{137} \mathrm{Cs}$ & ${ }^{238} \mathrm{Pu}$ & ${ }^{239,240 \mathrm{Pu}}$ \\
\hline 1981 & $3.6 \mathrm{E}-01$ & $\mathrm{NR}$ & $4.0 \mathrm{E}-01$ & NR & NR \\
\hline 1982 & $4.9 \mathrm{E}-01$ & $\mathrm{NR}$ & $3.2 \mathrm{E}-01$ & $N R$ & NR \\
\hline 1983 & $4.2 \mathrm{E}-01$ & $\mathrm{NR}$ & $1.7 \mathrm{E}-01$ & $\mathrm{NR}$ & NR \\
\hline 1984 & $2.2 \mathrm{E}-01$ & $1.4 \mathrm{E}-01$ & $1.6 \mathrm{E}-01$ & $1.4 \mathrm{E}-04$ & $9.8 \mathrm{E}-03$ \\
\hline 1985 & $2.4 \mathrm{E}-01$ & $5.6 \mathrm{E}-02$ & $2.7 \mathrm{E}-01$ & $2.1 \mathrm{E}-04$ & $3.0 \mathrm{E}-03$ \\
\hline 1986 & $2.6 \mathrm{E}-01$ & $7.3 \mathrm{E}-02$ & $8.7 \mathrm{E}-01$ & $3.2 \mathrm{E}-04$ & $5.8 \mathrm{E}-03$ \\
\hline 1987 & $2.8 \mathrm{E}-01$ & $2.0 \mathrm{E}-01$ & $1.1 \mathrm{E}+00$ & $1.6 \mathrm{E}-03$ & $1.8 \mathrm{E}-02$ \\
\hline 1988 & $1.9 \mathrm{E}-01$ & $1.5 \mathrm{E}-01$ & $9.4 \mathrm{E}-01$ & $6.2 \mathrm{E}-04$ & $8.2 \mathrm{E}-03$ \\
\hline 1989 & $2.2 \mathrm{E}-01$ & $8.8 \mathrm{E}-02$ & $6.7 \mathrm{E}-01$ & $6.2 \mathrm{E}-04$ & $1.4 \mathrm{E}-02$ \\
\hline 1990 & $9.3 \mathrm{E}-02$ & $9.8 \mathrm{E}-02$ & $9.5 \mathrm{E}-01$ & $6.2 \mathrm{E}-04$ & $2.9 \mathrm{E}-02$ \\
\hline 1991 & $1.1 \mathrm{E}-01$ & $7.0 \mathrm{E}-02$ & $9.8 \mathrm{E}-01$ & $3.1 \mathrm{E}-04$ & $9.1 \mathrm{E}-03$ \\
\hline
\end{tabular}

NR = Not Reported. 
Table H-5. Average Radionuclide Concentrations ( $\mathrm{pC} / \mathrm{g}$ ) Detected in 100-F Area Surface Soll Samples from 1981 to 1991.

\begin{tabular}{|c|c|c|c|c|c|}
\hline Year & ${ }^{60} \mathrm{Co}$ & ${ }^{90} \mathrm{Sr}$ & ${ }^{137} \mathrm{CS}$ & ${ }^{238} \mathrm{Pu}$ & ${ }^{239,240 \mathrm{Pu}}$ \\
\hline 1981 & $2.9 \mathrm{E}-01$ & $\mathrm{NR}$ & $6.0 \mathrm{E}-01$ & $\mathrm{NR}$ & NR \\
\hline 1982 & $1.4 \mathrm{E}+00$ & $\mathrm{NR}$ & $1.7 \mathrm{E}+00$ & $\mathrm{NR}$ & NR \\
\hline 1983 & $2.7 \mathrm{E}-01$ & $\mathrm{NR}$ & $8.6 \mathrm{E}-01$ & $\mathrm{NR}$ & NR \\
\hline 1984 & $1.1 \mathrm{E}+00$ & $4.4 \mathrm{E}-01$ & $7.0 \mathrm{E}-01$ & $5.0 \mathrm{E}-04$ & $1.0 \mathrm{E}-02$ \\
\hline 1985 & $3.0 \mathrm{E}-01$ & $2.9 \mathrm{E}-01$ & $8.3 \mathrm{E}-01$ & $6.5 \mathrm{E}-04$ & $9.8 \mathrm{E}-03$ \\
\hline 1986 & $2.3 \mathrm{E}-01$ & $2.7 \mathrm{E}-01$ & $7.4 \mathrm{E}-01$ & $3.8 \mathrm{E}-04$ & $1.1 \mathrm{E}-02$ \\
\hline 1987 & $1.9 \mathrm{E}-01$ & $2.4 \mathrm{E}-01$ & $5.5 \mathrm{E}-01$ & $6.4 \mathrm{E}-04$ & $9.3 \mathrm{E}-03$ \\
\hline 1988 & $1.7 \mathrm{E}-01$ & $1.6 \mathrm{E}-01$ & $6.2 \mathrm{E}-01$ & $1.3 \mathrm{E}-03$ & $1.4 \mathrm{E}-02$ \\
\hline 1989 & $1.3 \mathrm{E}-01$ & $1.1 \mathrm{E}-01$ & $4.3 \mathrm{E}-01$ & $8.0 \mathrm{E}-04$ & $1.3 \mathrm{E}-02$ \\
\hline 1990 & $4.4 \mathrm{E}-02$ & $1.1 \mathrm{E}-01$ & $6.5 \mathrm{E}-01$ & $5.2 \mathrm{E}-04$ & $1.1 \mathrm{E}-02$ \\
\hline 1991 & $4.9 \mathrm{E}-02$ & $1.6 \mathrm{E}-01$ & $8.3 \mathrm{E}-01$ & $8.6 \mathrm{E}-04$ & $1.4 \mathrm{E}-02$ \\
\hline
\end{tabular}

$N R=$ Not Reported.

Table H-6. Average Radionuclide Concentrations ( $\mathrm{pCi} / \mathrm{g}$ ) Detected in 100-H Area Surface Soll Samples from 1981 to 1991.

\begin{tabular}{|c|c|c|c|c|c|}
\hline Year & ${ }^{60} \mathrm{Co}$ & ${ }^{90} \mathrm{Sr}$ & ${ }^{137} \mathrm{CS}$ & ${ }^{238} \mathrm{Pu}$ & ${ }^{239,240 \mathrm{Pu}}$ \\
\hline 1981 & $1.5 \mathrm{E}-01$ & $\mathrm{NR}$ & $9.0 \mathrm{E}-02$ & $\mathrm{NR}$ & NR \\
\hline 1982 & $1.8 \mathrm{E}-01$ & $\mathrm{NR}$ & $3.4 \mathrm{E}-01$ & $\mathrm{NR}$ & NR \\
\hline 1983 & $1.6 \mathrm{E}-01$ & $\mathrm{NR}$ & $4.7 \mathrm{E}-01$ & $\mathrm{NR}$ & NR \\
\hline 1984 & $3.7 \mathrm{E}-01$ & $2.4 \mathrm{E}-01$ & $1.7 \mathrm{E}+00$ & $6.9 \mathrm{E}-04$ & $1.5 \mathrm{E}-02$ \\
\hline 1985 & $1.4 \mathrm{E}-01$ & $9.8 \mathrm{E}-02$ & $2.3 \mathrm{E}-01$ & $1.9 \mathrm{E}-04$ & $5.7 \mathrm{E}-03$ \\
\hline 1986 & $1.8 \mathrm{E}-01$ & $7.1 \mathrm{E}-02$ & $8.9 \mathrm{E}-01$ & $6.1 \mathrm{E}-04$ & $1.0 \mathrm{E}-02$ \\
\hline 1987 & $2.8 \mathrm{E}-01$ & $1.9 \mathrm{E}-01$ & $2.0 \mathrm{E}+00$ & $8.5 \mathrm{E}-04$ & $3.1 \mathrm{E}-02$ \\
\hline 1988 & $1.6 \mathrm{E}-01$ & $7.5 \mathrm{E}-02$ & $3.6 \mathrm{E}-01$ & $6.2 \mathrm{E}-04$ & $6.6 \mathrm{E}-03$ \\
\hline 1989 & $2.0 \mathrm{E}-01$ & $5.0 \mathrm{E}-02$ & $5.3 \mathrm{E}-01$ & $9.2 \mathrm{E}-04$ & $6.5 \mathrm{E}-03$ \\
\hline 1990 & $1.0 \mathrm{E}-01$ & $6.5 \mathrm{E}-02$ & $4.5 \mathrm{E}-01$ & $3.3 \mathrm{E}-01$ & $7.6 \mathrm{E}-03$ \\
\hline 1991 & $7.8 \mathrm{E}-02$ & $8.1 \mathrm{E}-02$ & $1.6 \mathrm{E}+00$ & $6.0 \mathrm{E}-04$ & $1.9 \mathrm{E}-02$ \\
\hline
\end{tabular}

$N R=$ Not Reported. 
Table H-7. Average Radionuclide Concentrations ( $\mathrm{pCi} / \mathrm{g}$ ) Detected in 100-K Area Surface Soll Samples from 1981 to 1991.

\begin{tabular}{|c|c|c|c|c|c|}
\hline Year & ${ }^{60} \mathrm{Co}$ & ${ }^{90} \mathrm{Sr}$ & ${ }^{137} \mathrm{CS}$ & ${ }^{238} \mathrm{Pu}$ & ${ }^{239,240} \mathrm{Pu}$ \\
\hline 1981 & $8.3 \mathrm{E}-01$ & $\mathrm{NR}$ & $4.4 \mathrm{E}+00$ & $\mathrm{NR}$ & $N R$ \\
\hline 1982 & $2.6 \mathrm{E}+01$ & $\mathrm{NR}$ & $8.8 \mathrm{E}-01$ & $\mathrm{NR}$ & NR \\
\hline 1983 & $5.5 \mathrm{E}+01$ & $\mathrm{NR}$ & $5.3 \mathrm{E}+01$ & $\mathrm{NR}$ & NR \\
\hline 1984 & $3.3 \mathrm{E}+00$ & $8.4 \mathrm{E}-01$ & $1.2 \mathrm{E}+01$ & $9.6 \mathrm{E}-04$ & $2.9 \mathrm{E}-02$ \\
\hline 1985 & $7.4 \mathrm{E}-01$ & $2.9 \mathrm{E}-01$ & $1.1 \mathrm{E}+00$ & $1.4 \mathrm{E}-03$ & $3.2 \mathrm{E}-02$ \\
\hline 1986 & $1.0 \mathrm{E}+00$ & $1.8 \mathrm{E}-01$ & $1.1 \mathrm{E}+00$ & $9.1 \mathrm{E}-04$ & $2.3 \mathrm{E}-02$ \\
\hline 1987 & $1.2 \mathrm{E}+00$ & $4.3 \mathrm{E}-01$ & $1.3 \mathrm{E}+00$ & $2.7 \mathrm{E}-03$ & $5.5 \mathrm{E}-02$ \\
\hline 1988 & $3.9 \mathrm{E}-01$ & $2.3 \mathrm{E}-01$ & $7.3 \mathrm{E}-01$ & $7.1 \mathrm{E}-04$ & $2.0 \mathrm{E}-02$ \\
\hline 1989 & $7.7 \mathrm{E}-01$ & $6.3 \mathrm{E}-01$ & $7.5 \mathrm{E}+00$ & $2.8 \mathrm{E}-03$ & $7.8 \mathrm{E}-02$ \\
\hline 1990 & $2.3 \mathrm{E}-01$ & $2.3 \mathrm{E}-01$ & $9.7 \mathrm{E}-01$ & $6.8 \mathrm{E}-04$ & $1.4 \mathrm{E}-02$ \\
\hline 1991 & $2.7 \mathrm{E}-01$ & $1.7 \mathrm{E}-01$ & $1.2 \mathrm{E}+00$ & $8.6 \mathrm{E}-04$ & $2.0 \mathrm{E}-02$ \\
\hline
\end{tabular}

NR $=$ Not Reported.

Table H-8. 200/600 Area Solls. (pCi/g)

\begin{tabular}{|c|c|c|c|c|c|c|c|c|c|c|c|c|}
\hline \multirow[b]{2}{*}{ Maximum } & \multicolumn{2}{|c|}{$737 \mathrm{cs}$} & \multicolumn{2}{|c|}{$90_{\mathrm{sr}}$} & \multicolumn{2}{|c|}{$239 / 240_{\mathrm{Pu}}$} & \multicolumn{2}{|c|}{$234 u$} & \multicolumn{2}{|c|}{$235 \mathrm{u}$} & \multicolumn{2}{|c|}{$238 \mathrm{~s}$} \\
\hline & 89 & $\begin{array}{l}\text { site } \\
1030\end{array}$ & 6.1 & $\begin{array}{l}\text { site } \\
1034\end{array}$ & 1.0 & $\begin{array}{l}\text { site } \\
1032\end{array}$ & 1.7 & $\begin{array}{l}\text { Site } \\
1088\end{array}$ & 0.14 & $\begin{array}{l}\text { S1te } \\
1089\end{array}$ & 1.6 & $\begin{array}{l}\text { site } \\
1088\end{array}$ \\
\hline Average & & 5.1 & & 0.7 & & 0.1 & & 0.7 & & 0.03 & & 0.7 \\
\hline $\begin{array}{l}\text { PWL Offeite } \\
\text { overage }\end{array}$ & & 0.43 & & 0.09 & & 0.007 & \multicolumn{6}{|c|}{1.5 total uranium } \\
\hline $\begin{array}{l}\text { Guide for } \\
\text { posting } \\
\text { surface } \\
\text { contamination }\end{array}$ & \multicolumn{2}{|c|}{20,000} & \multicolumn{2}{|r|}{600} & & 75 & \multicolumn{2}{|r|}{100} & \multicolumn{2}{|r|}{15} & & 50 \\
\hline
\end{tabular}


Table H-9. 300/400 Area Soll Sample. (pCi/g)

\begin{tabular}{|c|c|c|c|c|c|c|c|c|c|c|c|c|}
\hline \multirow[b]{2}{*}{ Maximum } & \multicolumn{2}{|c|}{${ }^{13} \mathrm{cs}$} & \multicolumn{2}{|c|}{${ }^{\infty} s r$} & \multicolumn{2}{|c|}{$200 / 200 \mathrm{pu}$} & \multicolumn{2}{|c|}{$2 x y$} & \multicolumn{2}{|c|}{ 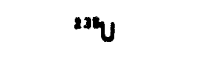 } & \multicolumn{2}{|c|}{290} \\
\hline & 0.51 & $\begin{array}{c}\text { site } \\
10 \\
s .7\end{array}$ & 0.21 & $\begin{array}{c}\text { site } \\
10 \\
s-8\end{array}$ & 0.15 & $\begin{array}{c}\text { site } \\
10 \\
8=4\end{array}$ & 20 & $\begin{array}{c}s 1 \text { te } \\
10 \\
s=4\end{array}$ & 0.88 & $\begin{array}{c}\text { site } \\
\text { id } \\
s-4\end{array}$ & 20 & $\begin{array}{c}s 1 t 0 \\
10 \\
s=4\end{array}$ \\
\hline Average & & 0.18 & & 0.06 & & 0.02 & & 3.1 & & 0.16 & & 2.9 \\
\hline $\begin{array}{l}\text { PNL East } \\
\text { Perimeter } \\
\text { si te average }\end{array}$ & & 0.43 & & 0.09 & & 0.007 & & & & 5 urar & $n$ to & \\
\hline $\begin{array}{l}\text { oulde for } \\
\text { posting } \\
\text { surface } \\
\text { contamination }\end{array}$ & 20 , & & & 800 & & 75 & & 100 & & 15 & & 50 \\
\hline
\end{tabular}

Table H-10. Average Radionuclide Concentrations ( $\mathrm{pCi} / \mathrm{g}$ ) Detected in Vegetation Samples near the 1301-N Liquid Waste Disposal Factilty from 1980 to 1991.

\begin{tabular}{|c|c|c|c|c|c|}
\hline Year & ${ }^{60} \mathrm{Co}$ & ${ }^{90} \mathrm{Sr}$ & ${ }^{137} \mathrm{CS}$ & ${ }^{238} \mathrm{Pu}$ & ${ }^{239,240 \mathrm{Pu}}$ \\
\hline 1980 & $4.0 \mathrm{E}+00$ & $\mathrm{NR}$ & $1.1 \mathrm{E}+00$ & $\mathrm{NR}$ & $\mathrm{NR}$ \\
\hline 1981 & $1.2 \mathrm{E}+01$ & $1.8 \mathrm{E}+00$ & $1.8 \mathrm{E}+00$ & NR & $7.1 \mathrm{E}-03$ \\
\hline 1982 & $1.6 \mathrm{E}+00$ & $1.2 \mathrm{E}-01$ & $2.6 \mathrm{E}-01$ & NR & $2.6 \mathrm{E}-03$ \\
\hline 1983 & $1.9 \mathrm{E}+00$ & $6.0 \mathrm{E}-01$ & $3.9 \mathrm{E}-01$ & $N R$ & $3.2 \mathrm{E}-03$ \\
\hline 1984 & $1.0 \mathrm{E}+00$ & $1.2 \mathrm{E}-01$ & $8.3 \mathrm{E}-02$ & NR & $8.5 \mathrm{E}-04$ \\
\hline 1985 & $1.7 \mathrm{E}+00$ & $1.9 \mathrm{E}+00$ & $1.0 \mathrm{E}-01$ & NR & $1.5 \mathrm{E}-03$ \\
\hline 1986 & $3.5 \mathrm{E}+00$ & $7.3 \mathrm{E}-02$ & $6.5 \mathrm{E}-01$ & $N R$ & $2.6 \mathrm{E}-03$ \\
\hline 1987 & $2.8 \mathrm{E}+00$ & $6.3 \mathrm{E}-02$ & $2.0 \mathrm{E}-01$ & $1.2 \mathrm{E}-03$ & $5.6 \mathrm{E}-03$ \\
\hline 1988 & $2.0 \mathrm{E}+00$ & $1.2 \mathrm{E}-01$ & $1.3 \mathrm{E}-01$ & $4.3 \mathrm{E}-04$ & $1.7 \mathrm{E}-03$ \\
\hline 1989 & $1.3 \mathrm{E}+00$ & $3.8 \mathrm{E}-02$ & $1.5 \mathrm{E}-01$ & $2.8 \mathrm{E}-04$ & $2.0 \mathrm{E}-03$ \\
\hline 1990 & $1.3 \mathrm{E}+00$ & $3.1 \mathrm{E}-02$ & $1.2 \mathrm{E}-01$ & $2.7 \mathrm{E}-04$ & $1.1 \mathrm{E}-03$ \\
\hline 1991 & $6.3 \mathrm{E}-01$ & $1.5 \mathrm{E}-02$ & $6.0 \mathrm{E}-05$ & $8.1 \mathrm{E}-05$ & $1.2 \mathrm{E}-03$ \\
\hline
\end{tabular}

NR $=$ Not reported. 
Table H-11. Average Radionucl lde Concentrations ( $\mathrm{pC} 1 / \mathrm{g}$ ) Detected in 100-N Vegetation Samples from 1980 to 1991.

\begin{tabular}{|c|c|c|c|c|c|}
\hline rear & ${ }^{60} \mathrm{Co}$ & $90 s r$ & $137 \mathrm{cs}$ & $238_{p u}$ & $238,240 \mathrm{Pu}$ \\
\hline 1980 & $1.0 E+00$ & NR & $2.8 E \cdot 01$ & MR & NR \\
\hline 1981 & $2.3 E+01$ & $5.8 \mathrm{E} \cdot 01$ & 7.1 E-01 & NR & $2.1 \mathrm{E} \cdot 02$ \\
\hline 1982 & $1.5 E+00$ & $2.0 \mathrm{E} \cdot 01$ & $1.3 E \times 01$ & NR & $7.8 E-03$ \\
\hline 1923 & $1.0 E+00$ & $2.9 * 01$ & 9.0 E.02 & MR & $8.6 \mathrm{E}-03$ \\
\hline 1984 & $4.6 E-01$ & $8.1 \varepsilon .02$ & $9.0=-02$ & NR & $1.3 \mathrm{E} .03$ \\
\hline 1985 & $1.4 E+00$ & $3.1 \mathrm{E} .02$ & $1.6 E-01$ & NR & $8.7 \mathrm{E} \cdot 04$ \\
\hline 1986 & $9.5 E-01$ & $2.2 \mathrm{E} .01$ & $7.9 E-01$ & NR & $1.1 \mathrm{E} .03$ \\
\hline 1987 & $7.0 \mathrm{E}-01$ & $2.6 \mathrm{E} \cdot 01$ & $9.4 E-02$ & $1.3 \mathrm{E} \cdot 04$ & $3.7 E \cdot 04$ \\
\hline 1988 & $8.0 \mathrm{E}-01$ & $2.5 \mathrm{E} \cdot 01$ & $1.6 \mathrm{E}=01$ & $1.7 E-04$ & $6.6 E-04$ \\
\hline 1989 & $3.2=01$ & $6.8 \mathrm{E} \cdot 02$ & $1.5 E .01$ & $1.1 E-04$ & $8.7 \mathrm{E} \cdot 04$ \\
\hline 1990 & $1.1 E-01$ & $9.3 \in .03$ & $3.6 \mathrm{E}-02$ & $<9.6 E .05$ & $1.7 E=04$ \\
\hline 1991 & $1.3 \mathrm{E} \cdot 01$ & $9.4 \mathrm{E} \cdot 03$ & $3.4 E-03$ & $1.6 E-04$ & $2.5=-03$ \\
\hline
\end{tabular}

NR - Not reported.

Table H-12. Radionuclide Concentrations ( $\mathrm{pCi} / \mathrm{g}$ ) Detected in $\mathrm{N}$-Springs Vegetation Samples from 1980 to 1991.

\begin{tabular}{|c|c|c|c|c|c|}
\hline Yoar & ${ }^{60} \mathrm{Co}$ & ${ }^{90} \mathrm{sr}$ & $137 \mathrm{Cs}$ & $238 \mathrm{Pu}$ & $239,240 \mathrm{pu}$ \\
\hline 1980 & $3.6 t+00$ & NR & $4.4 E \cdot 01$ & NR & NR \\
\hline 1981 & $3.3 E+00$ & $2.0 E+02$ & NR & NR & $3.7 \varepsilon-03$ \\
\hline 1982 & $2.8 E+00$ & $4.8 E+02$ & NR & NR & $8.3 \mathrm{E} \cdot 03$ \\
\hline 1983 & $3.0 E+00$ & $3.3 E+02$ & 4.0 E-02 & NR & $8.0 \mathrm{E}-03$ \\
\hline 1984 & NR & NR & NR & MR & NR \\
\hline 1985 & $1.2 E+00$ & $4.2 \mathrm{E}+02$ & $1.7 E-01$ & NR & $4.4 E-04$ \\
\hline 1986 & $1.1 E+00$ & $2.2 E+02$ & $2.1 E \cdot 01$ & NR & $4.2 \mathrm{E}-04$ \\
\hline 1987 & $9.0 \varepsilon-01$ & $2.9 E+02$ & $1.1 \mathrm{E}-01$ & $<1.3 \mathrm{E}-04$ & 7.6 E-04 \\
\hline 1988 & $1.4 E+00$ & $1.2 E+02$ & $2.0 E-01$ & $8.5 E-05$ & $2.0 \mathrm{E} \cdot 04$ \\
\hline 1989 & $4.3 \mathrm{E}-01$ & $8.0 E+01$ & $1.5 E-01$ & $1.1 E-03$ & $4.5 \mathrm{E} \cdot 04$ \\
\hline 1990 & NS & NS & NS & MS & NS \\
\hline 1991 & $1.5 E-01$ & $3.1 E+01$ & $1.6 E-01$ & $1.2 E-04$ & $2.9 E-03$ \\
\hline
\end{tabular}

NR $=$ Not reported.

NS - No sample results. 
Table H-13. Average Radionuclide Concentrations ( $\mathrm{pCl} / \mathrm{g}$ ) Detected in 100-B/C Area Vegetation Samples from 1981 to 1991.

\begin{tabular}{|c|c|c|c|c|c|}
\hline Year & ${ }^{60} \mathrm{Co}$ & $80_{s r}$ & $137 \mathrm{cs}$ & $238 \mathrm{pu}$ & $39,240 \mathrm{Pu}$ \\
\hline 1981 & $3.6 E+00$ & NR & $3.6 e .01$ & NR & MR \\
\hline 1982 & $1.9 E .01$ & NR & $1.1 E-01$ & NR & NR \\
\hline 1903 & $1.8=01$ & NR & $8.0=-02$ & NR & NR \\
\hline 1984 & $1.3=-01$ & $1.4 E+00$ & $8.7 \in-02$ & $2.4:-04$ & $6.0=-04$ \\
\hline 1985 & $4.6=.01$ & $1.4 E+00$ & $1.2 E \cdot 01$ & $2.5 \mathrm{E} .04$ & $1.0 \times \cdot 03$ \\
\hline 1986 & $2.5=.01$ & $2.0 \mathrm{E}-01$ & $2.8 E+00$ & $2.5 \mathrm{e.05}$ & $6.2 \mathrm{E} \cdot 04$ \\
\hline 1987 & $1.5<.01$ & $2.3 \mathrm{E} \cdot 01$ & $1.0 \mathrm{E} .01$ & $4.6 \mathrm{E}-04$ & $6.5 \div \cdot 04$ \\
\hline 1988 & $3.5 \times-01$ & $2.6 \mathrm{E} \cdot 01$ & $2.1 \mathrm{E} .09$ & $1.4 \mathrm{E} \cdot 06$ & $3.1 E .04$ \\
\hline 1989 & $3.2=01$ & $1.6 E .01$ & $1.8 \mathrm{E} \cdot 01$ & $6.5 \varepsilon .05$ & $2.4 \varepsilon-04$ \\
\hline 1990 & $4.5 E .02$ & $9.1 E .02$ & $6.7 E .02$ & $<2.5=05$ & $3.0:-04$ \\
\hline 1991 & $5.7 \in .02$ & $8.3 \mathrm{E} \cdot 02$ & $1.8 E \cdot 01$ & $2.9 \mathrm{E} .05$ & $1.1 E \cdot 03$ \\
\hline
\end{tabular}

NR - Not reported.

Table H-14. Average Radionuclide Concentrations ( $\mathrm{pCl} / \mathrm{g}$ ) Detected In 100-D/DR Area Vegetation Samples from 1981 to 1991.

\begin{tabular}{|c|c|c|c|c|c|}
\hline Year & ${ }^{60} \mathrm{Co}$ & ${ }^{90} \mathrm{Sr}$ & ${ }^{137} \mathrm{Cs}$ & ${ }^{238} \mathrm{Pu}$ & $239,240 \mathrm{Pu}$ \\
\hline 1981 & $1.2 E+00$ & NR & $1.6 \mathrm{E}-01$ & NR & NR \\
\hline 1982 & $1.1 E-01$ & NR & $2.7 E+00$ & NR & NR \\
\hline 1983 & $9.5 E-02$ & NR & $1.4 E-01$ & NR & NR \\
\hline 1984 & $2.1 E-01$ & $2.8 E-01$ & $1.7 E+00$ & $1.8 E-03$ & $5.8 E-04$ \\
\hline 1985 & $2.4 E-01$ & $6.9 E-02$ & $6.8 E-01$ & $1.2 E-04$ & 7.0 E-04 \\
\hline 1986 & $2.7 E-01$ & $1.5 E-01$ & $1.7 E+00$ & 0.0 & $3.1 E-04$ \\
\hline 1987 & $2.5 E-01$ & $9.5 E-02$ & $6.3 \mathrm{E}-01$ & $1.6 E-04$ & $2.8 E-04$ \\
\hline 1988 & $2.8 E-01$ & $1.8 E-01$ & $9.6 E-02$ & $3.8 E-05$ & $1.9 E-04$ \\
\hline 1989 & $2.6 E-01$ & $1.5 \mathrm{E}-01$ & $2.8 E-01$ & $9.6 E-05$ & $1.2 E-04$ \\
\hline 1990 & $6.1 E-02$ & $9.5 E-02$ & $6.2 E-01$ & $<2.4 \quad E-05$ & $2.6 E-04$ \\
\hline 1991 & $5.7 E-02$ & $8.3 E-02$ & $1.8 E-01$ & $2.9 E-05$ & $1.1 E-03$ \\
\hline
\end{tabular}

NR = Not reported. 
Table H-15. Average Radionuclide Concentrations ( $\mathrm{pC} 1 / \mathrm{g}$ ) Detected in 100-F Area Vegetation Samples from 1981 to 1991.

\begin{tabular}{|c|c|c|c|c|c|}
\hline Year & ${ }^{60} \mathrm{CO}$ & ${ }^{90} \mathrm{Sr}$ & ${ }^{137} \mathrm{CS}$ & ${ }^{238} \mathrm{PU}$ & ${ }^{239,240 \mathrm{PU}}$ \\
\hline 1981 & $9.2 \mathrm{E}-01$ & $\mathrm{NR}$ & $2.2 \mathrm{E}+00$ & $\mathrm{NR}$ & $\mathrm{NR}$ \\
\hline 1982 & $1.6 \mathrm{E}-01$ & $\mathrm{NR}$ & $7.9 \mathrm{E}-01$ & $\mathrm{NR}$ & $\mathrm{NR}$ \\
\hline 1983 & $2.8 \mathrm{E}-01$ & $\mathrm{NR}$ & $1.0 \mathrm{E}+00$ & $\mathrm{NR}$ & $\mathrm{NR}$ \\
\hline 1984 & $2.2 \mathrm{E}+00$ & $7.6 \mathrm{E}+00$ & $2.0 \mathrm{E}+01$ & $4.9 \mathrm{E}-04$ & $3.9 \mathrm{E}-03$ \\
\hline 1985 & $3.3 \mathrm{E}-01$ & $1.4 \mathrm{E}+00$ & $5.8 \mathrm{E}-01$ & $4.9 \mathrm{E}-05$ & $5.3 \mathrm{E}-04$ \\
\hline 1986 & $1.7 \mathrm{E}-01$ & $9.3 \mathrm{E}-02$ & $1.1 \mathrm{E}+00$ & $8.3 \mathrm{E}-05$ & $1.2 \mathrm{E}-04$ \\
\hline 1987 & $2.4 \mathrm{E}-01$ & $1.8 \mathrm{E}-01$ & $1.8 \mathrm{E}-01$ & $3.8 \mathrm{E}-04$ & $1.7 \mathrm{E}-04$ \\
\hline 1988 & $9.9 \mathrm{E}-01$ & $8.9 \mathrm{E}-02$ & $3.1 \mathrm{E}-01$ & $3.3 \mathrm{E}-05$ & $1.1 \mathrm{E}-04$ \\
\hline 1989 & $2.8 \mathrm{E}-01$ & $9.6 \mathrm{E}-02$ & $2.0 \mathrm{E}+00$ & $5.2 \mathrm{E}-05$ & $1.4 \mathrm{E}-04$ \\
\hline 1990 & $5.0 \mathrm{E}-02$ & $2.7 \mathrm{E}-01$ & $4.2 \mathrm{E}-01$ & $<4.9 \mathrm{E}-05$ & $3.6 \mathrm{E}-04$ \\
\hline 1991 & $8.2 \mathrm{E}-02$ & $2.9 \mathrm{E}-01$ & $3.8 \mathrm{E}-01$ & $2.9 \mathrm{E}-05$ & $7.0 \mathrm{E}-03$ \\
\hline
\end{tabular}

NR = Not reported.

Table H-16. Average Radionuclide Concentrations ( $\mathrm{pCi} / \mathrm{g}$ ) Detected in 100-H Area Vegetation Samples from 1981 to 1991.

\begin{tabular}{|c|c|c|c|c|c|}
\hline Year & ${ }^{60} \mathrm{Co}$ & $90 \mathrm{gr}$ & ${ }^{137} \mathrm{Cs}$ & ${ }^{238}{ }_{\mathrm{Pu}}$ & $239,240_{\mathrm{Pu}}$ \\
\hline 1981 & $6.8 E-01$ & MR & $1.5 E .01$ & NR & NR \\
\hline 1982 & Mn & NR & NR & NR & MR \\
\hline 1983 & $1.3=-01$ & NR & 9.0 E.02 & MR & NR \\
\hline 1984 & $1.8 \mathrm{E}-01$ & $2.0 E+00$ & $1.3 E .01$ & $2.0 E-04$ & $1.7 \mathrm{E.03}$ \\
\hline 1985 & $2.0=-01$ & $6.0 \mathrm{E} .02$ & $4.5 \mathrm{E} \cdot 02$ & $1.0 \mathrm{E} \cdot 04$ & $5.1 E .04$ \\
\hline 1986 & $2.2=-01$ & $5.3 E-01$ & $1.3 E+00$ & $1.3=-04$ & 4.4 E-05 \\
\hline 1987 & $2.6 \mathrm{E} \cdot 01$ & $2.6 E .01$ & $1.0 \mathrm{E} \cdot 01$ & 3.5 E.05 & $2.7 E-04$ \\
\hline 1988 & 9.0 E-01 & 3.9 E.01 & $1.5 \mathrm{E} \cdot 01$ & $2.0 E-04$ & $1.5 E-04$ \\
\hline 1989 & $6.58-01$ & $5.2 E .02$ & $2.1 \mathrm{E}-01$ & $8.5 E-05$ & $1.5=-04$ \\
\hline 1990 & $<1.3 E-01$ & $1.1 E .02$ & $6.6 E-02$ & $<9.4 E-04$ & $3.0 \mathrm{E} \cdot 04$ \\
\hline 1991 & $<2.8 \varepsilon-02$ & $5.7 E-02$ & $3.0 \mathrm{E}-02$ & $4.5 \mathrm{E} \cdot 05$ & $5.7 E-04$ \\
\hline
\end{tabular}

NR * Not reported. 
Table H-17. Average Radionuclide Concentrations ( $\mathrm{pCl} / \mathrm{g}$ ) Detected in 100-K Area Vegetation Samples from 1981 to 1991.

\begin{tabular}{|c|c|c|c|c|c|}
\hline rear & ${ }^{60} \mathrm{CO}$ & $90 \mathrm{sr}$ & $137 \mathrm{Cs}$ & $238_{p u}$ & $239,240_{\mathrm{Pu}}$ \\
\hline 1981 & $1.2 \pm+00$ & WR & $1.0 \div .01$ & Mn & wh \\
\hline 1982 & $2.4 \div .01$ & NR & $9.7=01$ & MR & Nh \\
\hline 1903 & $1.5=01$ & NR & $2.5 \times .01$ & NR & NA \\
\hline 1904 & $1.8 \times 01$ & $1.3 E+00$ & $1.3=01$ & $2.9 \times .04$ & $0.9 \times-04$ \\
\hline 1905 & $4.6 \times \cdot 01$ & $3.9=01$ & $1.3:-01$ & $1.9 \cdot 04$ & $7.1=0.04$ \\
\hline 1928 & $2.8 \times \cdot 01$ & 4.08 .01 & $1.3 \varepsilon+00$ & $2.5=06$ & 7.01 .04 \\
\hline 1987 & 2.38 .01 & $1.3 \subset+00$ & $1.1 \times .01$ & $1.9 \mathrm{n} \cdot 04$ & $2.2 \div .06$ \\
\hline 1988 & $4.9 \mathrm{e}-01$ & $1.2 \varepsilon+00$ & $1.8 \times \cdot 01$ & $3.2 \in .05$ & $3.8=04$ \\
\hline 1898 & $3.1<.01$ & $1.3 \varepsilon+00$ & $1.6 \mathrm{E} \cdot 01$ & $1.1=.04$ & $1.5 \mathrm{E} \cdot 04$ \\
\hline 1990 & $6.5=\cdot 02$ & $0.0 \mathrm{E}+00$ & $4.1 E .02$ & $11.7=04$ & 2.58 .06 \\
\hline 1991 & $0.3 \div .02$ & $4.1 \div .01$ & $7.6 \div .02$ & $3.2=0.05$ & $5.9 \square \cdot 04$ \\
\hline
\end{tabular}

Mh - Not reported.

Table H-18. 200 Area Vegetation (pCl/g).

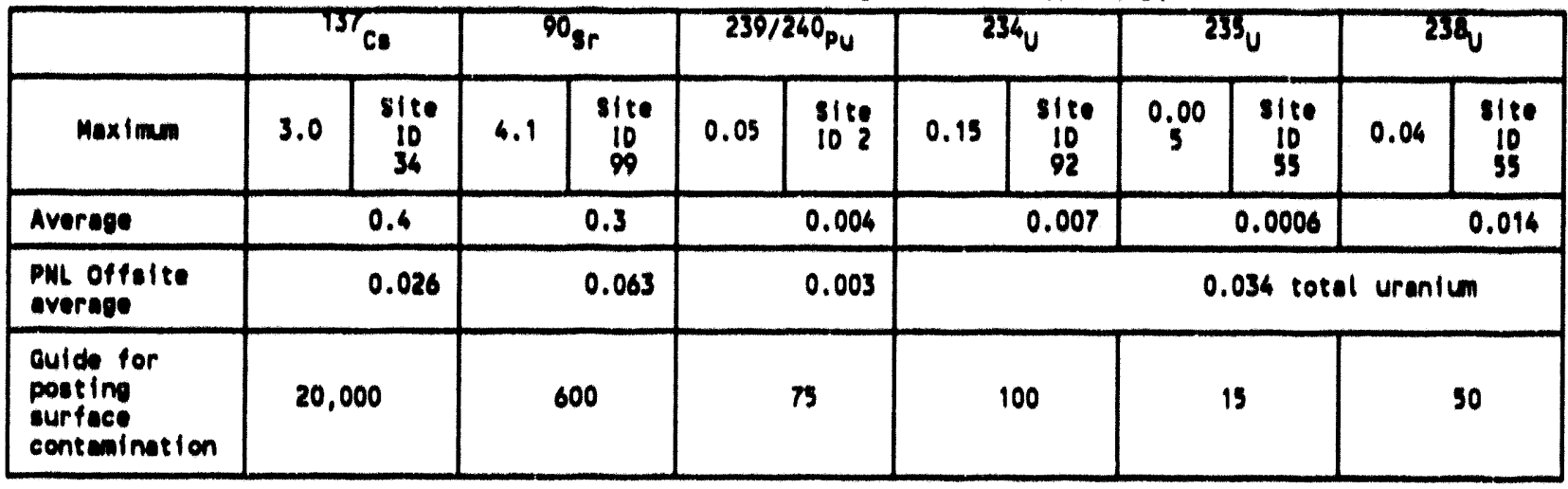


WHC-EP-0620

\section{APPENOIX I}

PLANT SPECIES OBSERVED AT 100 AREAS OPERABLE UNITS 
WHC-EP-0620

Th1s page intentionally left blank. 
WHC-EP-0620

Figure 1-1, 100 Areas Operable Units.

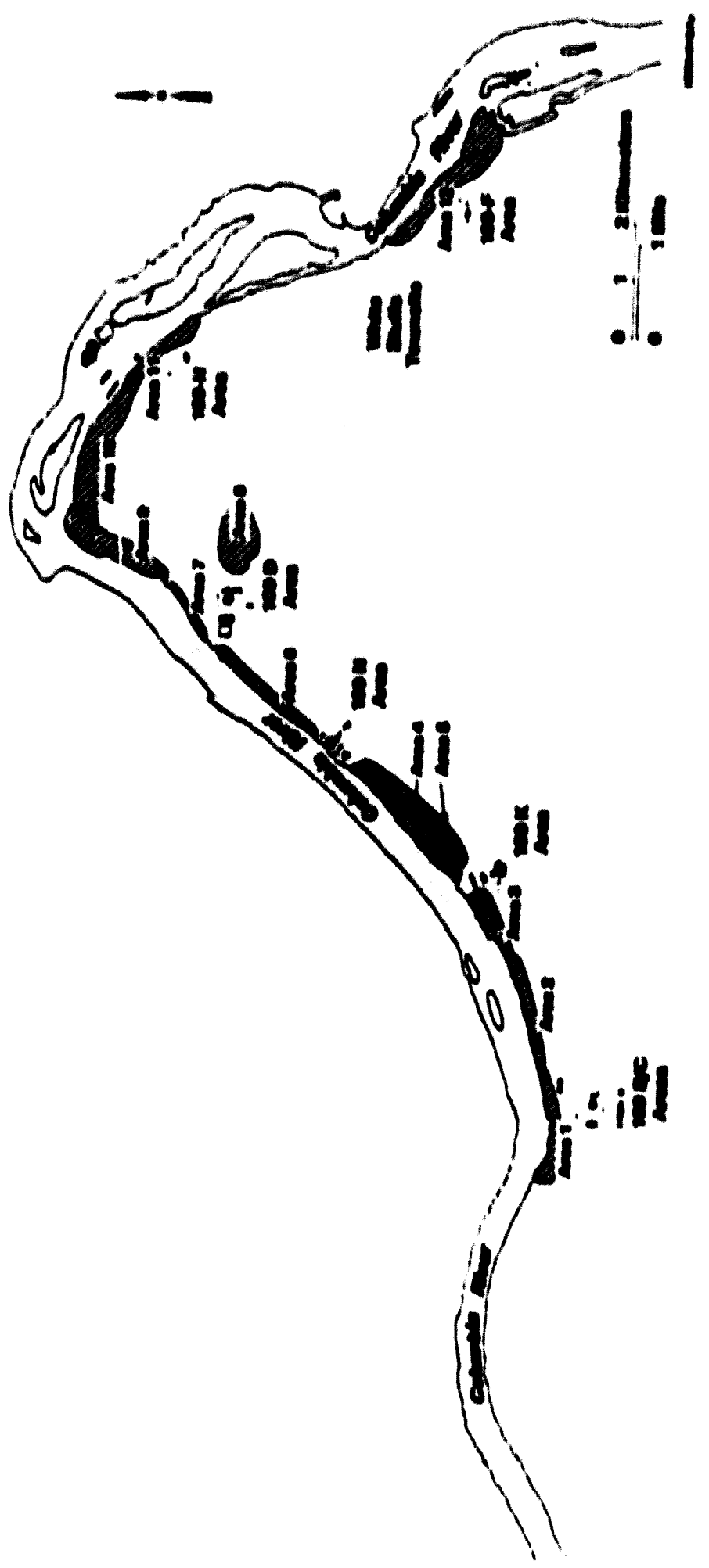


WHC-EP-0620

Table 1-1. Spectes Observed in Vicintey of 100-8C Area. (sheet 1 of 2)

\begin{tabular}{|c|c|c|}
\hline Species & Fantly & Common name \\
\hline Achillea atllofollum & Asteraceae & yarrow \\
\hline Artentsta campestris & Asteraceae & Pacific sage \\
\hline Artenista ludovictane & Asteracea & pratrte sagebrush \\
\hline Artenista tridentata & Asteraceas & blg sagebrush \\
\hline Aster hesportus & Asteraceas & mastern marsh aster \\
\hline Chrysothannus nauscosus & Asteraceae & gray rabblebrush \\
\hline Centaurea repons & Asteracese & Russtan knapwoed \\
\hline Cirstum arvense & Asteracese & Canade thistle \\
\hline Correopsts atkinsontane & Asteracens & Columbta tickseed \\
\hline Galliardia aristato & Asteraceas & blanket flower \\
\hline Gnaphallum palustre & Asteraceas & lowl and cudweed \\
\hline Grindella columblana & Asteracene & Columbla River gumuesed \\
\hline Helentun autumnale & Asternecese & sneezoweod \\
\hline Soltdago canadensis & Asteraceas & madow goldenrod \\
\hline Solldego occidentalis & Asteracease & western goldenrod \\
\hline Xanthlum strumarlum & Asteraceas & cocklobur \\
\hline Ansinckta Iycopsoides & Boraginaceas & Plddleneck \\
\hline Descurainie pinnata & Brasstcaceae & western tansymustard \\
\hline Descurainta sophta & Brasstcacene & fll xweed \\
\hline Lepidiun porfollatum & Brass leaceas & clasping pepperweed \\
\hline Lesqueralla douglasll & Brasstcacoae & Columbla bladderpod \\
\hline Rorlppe columblas & Brassicacose & Columbla yelloweress \\
\hline sisymbrlum altissimum & Brass fcacese & Jim HIll's tumblemustard \\
\hline Cerastium viscosum & Caryophyllacese & stlcky chlckweed \\
\hline Juntperus scopulorum & Cuprossacoae & Rocky Mountain Juntpor \\
\hline Carex douglastl & Cyporaceas & Douglas' sodge \\
\hline Carex dense & Cyporacese & Dense sedge \\
\hline Carex Ienticularis & Cyperaceae & Kollogg's sedge \\
\hline Glycyrrhiza lepidota & Fabaceae & Ilcorlce \\
\hline
\end{tabular}


Table 1-1. Spectes Observed in Vicinity of 100-BC Area. (sheet 2 of 2)

\begin{tabular}{|c|c|c|}
\hline Spectes & Fantly & Common name \\
\hline Melllotus alba & Fabaceae & white sweetclover \\
\hline Lupinus wyethit & Fabaceace & Wyeth's lupine \\
\hline Erodtun cicutariun & Gerantaceae & storksbill \\
\hline Hypericun perforatum & Hypertcacuae & Klamath weed \\
\hline Juncus balticus & Juncaceae & Baltic rush \\
\hline Juncus spp. & Juncaceae & rush \\
\hline Sphaeraleen munroana & Malvaceae & Munro's globemsllow \\
\hline Horus alba & Moraceae & white mulberry \\
\hline Eplloblum watsonil & Onagraceae & Watson's wfllow herb \\
\hline Oenothera strigosa & Onagraceae & common evening primrose \\
\hline Agropyron dasytachyum & Poscene & thickspike wheatgrass \\
\hline Agrostis alba & Poscease & radtop bentgrass \\
\hline Bromus tectorum & Poncene & choatgrass \\
\hline Phalaris arundinacea & Poscone & Reed canarygrass \\
\hline Pou sandbergif & Porceas & Sandberg's bluegrass \\
\hline Sttanion hystrix & Porceae & bottlebrush grass \\
\hline Sporobolus cryptandrus & Porceare & sand dropseed \\
\hline Polygonum persicarta & Polygonaceare & heartwead \\
\hline Potentllla anserina & Rosaceae & common sllverweed \\
\hline Sallx SPP. & Salleacene & wlllow \\
\hline Gratiola neglecta & Scrophularlaceae & Amertcan hedge-hyssop \\
\hline Verbascun thapsus & Scrophulartaceae & common mulletn \\
\hline Collinsta parviflora & Scrophularlaceae & small blue-eyed Mary \\
\hline Limosella aquatle (acaulis) & Scrophularlaceae & southern mudwort \\
\hline Lindernia anagallidea & Scrophulariaceae & false plmpernel \\
\hline Verontca peregina & Scrophulariaceae & purslane speedwell \\
\hline VImus punilia & UImaceae & Sibertan elm \\
\hline Verbena bracteata & Verbenaceae & bracted verbena \\
\hline
\end{tabular}

spp. - spectes, more than one. 
WHC-EP-0620

Table 1-2. Spectes Observed along Shorel the between 100-8 and Allard Pumphouse. (sheet 1 of 2)

\begin{tabular}{|c|c|c|}
\hline Spectes & Family & Common name \\
\hline Apocynum cennebinum & Apocynaceae & Common dogbane \\
\hline Asclepies speciose & Asclepiadaceae & Showy mil kweed \\
\hline Achillee nillefollun & Astereceace & Yarrow \\
\hline Antennerie umbrinelle & Asteracece & Umber pussytoes \\
\hline Artenisie campestris & Astericeae & Paciffc sage \\
\hline Artenisle lindlexene & Astericeae & Columbla River mugwort \\
\hline Artemiste ludovictene & Asterecese & Prairle sage \\
\hline Arteniste tridentete & Asteracece & Bie sagebrush \\
\hline Aster hesperius & Asteraceae & Western marsh aster \\
\hline Centeuree diffuse & Asteraceae & Diffuse knapweed \\
\hline Centauree repens & Asteraceae & Russtan knapweed \\
\hline Cichoriun intybus & Asteraceae & Chicory \\
\hline Chrysothemnus neuseosus & Asteracese & Gray rabbitbrush \\
\hline Coreopsis atkinsoniene & Asteraceae & Tickseed \\
\hline Galllerdie eristate & Asteraceae & Blanketflower \\
\hline Grindelie columbiene & Asteracece & Columbla River gumweed \\
\hline Helenium eutumnale & Asteraceae & Sneezeweed \\
\hline Rorippe islendice & Brassicaceae & Western yelloweress \\
\hline Rorippe obtuse & Brasstcaceae & Blunt leaf yelloweress \\
\hline sisymbriun eltissimum & Brassicaceae & Jim Hill Mustard \\
\hline Dianthus ermerte & Caryophyllaceae & Grass pink \\
\hline Selsole kall & Chenopodiaceae & Russian thistle \\
\hline Convolvulus ervensis & Convolvulaceae & Field bindweed \\
\hline Carex dens: & Cyperaceae & Dense sedge \\
\hline Carex sp. & Cyperaceae & Sedge \\
\hline Eleocherts palustris & Cyperaceae & Common spikerush \\
\hline Melllotus albe & Fabaceae & White sweetclover \\
\hline Irifollum repens & Fabaceae & white clover \\
\hline Hypericum perforatum & Hypericaceae & St. Johnswort \\
\hline
\end{tabular}


Table I-2. Species Observed along Shoreline between 100-B and Allard Pumphouse. (sheet 2 of 2)

\begin{tabular}{|l|l|l|}
\hline \multicolumn{1}{|c|}{ Species } & \multicolumn{1}{|c|}{ Family } & \multicolumn{1}{c|}{ Common name } \\
\hline Allium ssp. & Liliaceae & Onion \\
\hline Asparagus officinale & Liliaceae & Asparagus \\
\hline Lythrum salicaria & Lythraceae & Purple loosestrife \\
\hline Morus alba & Moraceae & Mulberry \\
\hline Epilobium watsonii & Onagraceae & Watson's willowherb \\
\hline Plantago lanceolata & Plantaginaceae & English plantain \\
\hline Plantago major & Plantaginaceae & Common plantain \\
\hline Agropyron dasytachyum & Poaceae & Thicisspike wheatgrass \\
\hline Agrostis scabrella & Poaceae & ticklegrass \\
\hline Bromus tectorum & Poaceae & Cheatgrass \\
\hline Phaiaris arundinacea & Poaceae & Reed canarygrass \\
\hline Poa pratensis & Poaceae & Kentucky bluegrass \\
\hline Sporobolus cryptandrus & Poaceae & Sand dropseed \\
\hline Polygonum persicaria & Polygonaceae & Doorweed \\
\hline Rumex salicifolius & Polygonaceae & Willow dock \\
\hline Ranunculus flammula & Ranunculaceae & Creeping buttercup \\
\hline Salix exigua & Salicaceae & Coyote willow \\
\hline Verbascum thapsus & Scrophulariaceae & Wooly mullein \\
\hline Ulmus pumila & Ulmaceae & Siberian elm \\
\hline Verbena bracteata & Verbenaceae & Bracted verbena \\
\hline Verbena hastata & Verbenaceae & Blue verbena \\
\hline
\end{tabular}

spp. = species, more than one. 
Table 1-3. Species Observed between Allard Pumphouse and 100-K Area. (sheet 1 of 4 )

\begin{tabular}{|c|c|c|}
\hline Spectes & Family & Common name \\
\hline Lomatium canbyi & Aplaceae & Canby's Desertparsley \\
\hline Lomatium grayi & Aplaceae & Gray's Desertparsley \\
\hline Apocynum cannabinum & Apocynaceae & Common dogbane \\
\hline Asclepias speciosa & Asclepladaceae & Showy milkweed \\
\hline Achillea millefolium & Asteraceae & Yarrow \\
\hline Antennaria umbrinella & Asteraceae & Umber pussytioes \\
\hline Artemisia campestris & Asteraceae & Pacific Sage \\
\hline Artemisia lindleyana & Asteraceae & Columbia river mugwort \\
\hline Artemisia Iudoviciana & Asteraceae & Prairie Sage \\
\hline Artemisia tridentata & Asteraceae & Big sagebrush \\
\hline Aster hesperius & Asteraceae & Western marsh aster \\
\hline Centaurea diffusa & Asteraceae & Diffuse knapweed \\
\hline Cichorium intybus & Asteraceae & Chicory \\
\hline Chrysothamnus nauseosus & Asteraceae & Gray rabbitbrush \\
\hline Cirsium arvense & Asteraceae & Canada thistle \\
\hline Conyza canadensis & Asteraceae & Horseweed \\
\hline Coreopsis atkinsoniana & Asteraceae & Columbia tickseed \\
\hline Gaillardia aristata & Asteraceae & Blanketflower \\
\hline Grindelia columbiana & Asteraceae & Columbia river gumweed \\
\hline Helenium autumnale & Asteraceae & Sneezeweed \\
\hline Lactuca serriola & Asteraceae & Prickly lettuce \\
\hline Solidago graminifolia & Asteraceae & Bushy goldenrod \\
\hline Solidago ssp. & Asteraceae & goldenrod \\
\hline Taraxacum officinale & Asteraceae & Dandelion \\
\hline Tragopogon dubius & Asteraceae & Salsify \\
\hline Xanthium strumarium & Asteraceae & Cocklebur \\
\hline Amsinckia lycopsoides & Boraginaceae & Tarweed fiddleneck \\
\hline Myosotis laxa & Boraginaceae & Smal1 forget-me-not \\
\hline Cardamine pennsylvanica & Brassicaceae & Pennsylvania bittercress \\
\hline
\end{tabular}


Table 1-3. Species Observed between Allard Pumphouse and 100-K Area. (sheet 2 of 4 )

\begin{tabular}{|c|c|c|}
\hline Spectes & Famtly & Common name \\
\hline Descurainia pinnata & Brassicaceae & Tansymustard \\
\hline Draba verna & Brassicaceae & Spring whitlowgrass \\
\hline Lepidium perfoliatum & Brassicaceae & Clasping pepperweed \\
\hline Rorippa curvisiliqua & Brassicaceae & Western yellowcress \\
\hline Rorippa islandica & Brassicaceae & Marsh yellowcress \\
\hline Rorippa obtusa & Brassicaceae & Bluntleaf yellowcress \\
\hline sisymbrium altissimum & Brassicaceae & Jim Hill mustard \\
\hline Cerastium vulgatum & Caryophyllaceae & Common chickweed \\
\hline Dianthus armeria & Caryophyllaceae & Grass pink \\
\hline Holosteum umbellatum & Caryophyl1 aceae & Jagged chickweed \\
\hline Salsola kali & Chenopodiaceae & Russian thistle \\
\hline Convolvulus arvensis & Convolvulaceae & Fleld bindweed \\
\hline Juniperus scopulorum & Cupressaceae & Rocky Mountain juniper \\
\hline Carex aperta & Cyperaceae & Columbia sedge \\
\hline Carex densa & Cyperaceae & Dense sedge \\
\hline Carex ssp. & Cyperaceae & sedges \\
\hline Eleocharis palustris & Cyperaceae & Common spikerush \\
\hline Scirpus americanus & Cyperaceae & Threesquare bulrush \\
\hline Equisetum ssp. & Equisetaceae & Horsetail \\
\hline Lupinus wyethii & Fabaceae & Wyeth's lupine \\
\hline Melilotus alba & Fabaceae & White sweetclover \\
\hline Psoralea lanceolata & Fabaceae & Dune scurfpea \\
\hline Robinia psuedo-acacia & Fabaceae & Black Locust \\
\hline Irifolium repens & Fabaceae & White clover \\
\hline Erodium cicutarium & Geraniaceae & Cranes bill \\
\hline Myriophyllum spicatum & Haloragaceae & Spiked water milfoil \\
\hline Hypericum perfoliatum & Hypericaceae & St. Johnswort \\
\hline Iris missouriensis & Iridaceae & Western blueflag \\
\hline
\end{tabular}


WHC-EP-0620

Table 1-3. Species Observed between Allard Pumphouse and 100-K Area. (sheet 3 of 4 )

\begin{tabular}{|l|l|l|}
\hline \multicolumn{1}{|c|}{ Species } & \multicolumn{1}{|c|}{ Famlly } & \multicolumn{1}{c|}{ Common name } \\
\hline Juncus articulatus & Juncaceae & Jointed rush \\
\hline Juncus balticus & Juncaceae & Baltic rush \\
\hline Juncus tenuis & Juncaceae & Slender rush \\
\hline Triglochin palustre & Juncaginaceae & March arrowgrass \\
\hline Allium cernuum & Lillaceae & Nodding onion \\
\hline Allium robinsonif & Llliaceae & Robinson's onton \\
\hline Allium schoenoprasum & Lillaceae & Chives \\
\hline Asparagus officinale & Lillaceae & Asparagus \\
\hline Lythrum salicaria & Lythraceae & Purple Loosestrife \\
\hline Sphaeralcea munroana & Malvaceae & Globemallow \\
\hline Morus alba & Moraceae & Mulberry \\
\hline Epilobium watsonii & Onagraceae & watson's willowherb \\
\hline Plantago lanceolata & Plantaginaceae & English plantain \\
\hline Plantago major & Plantaginaceae & Common plantain \\
\hline Agropyron dasytachyum & Poaceae & Thickspike wheatgrass \\
\hline Agrostis scabra & Poaceae & Ticklegrass \\
\hline Bromus tectorum & Poaceae & Cheatgrass \\
\hline Festuca arundinacea & Poaceae & Tall fescue \\
\hline Phalaris arundinacea & Poaceae & Reed canarygrass \\
\hline Poa bulbifera & Poaceae & Bulbous bluegrass \\
\hline Poa nevadensis & Poaceae & Nevada bluegrass \\
\hline Poa pratensis & Poaceae & Kentucky bluegrass \\
\hline Poa sandbergii & Poaceae & Sandberg's bluegrass \\
\hline Sporobolus cryptandrus & Poaceae & Sand dropseed \\
\hline Collomia linearis & Polemoniaceae & Narrowleaf collomia \\
\hline Phlox longifolia & Polemoniaceae & Longleaf phlox \\
\hline Polygonum persicaria & Polygonaceae & Heartweed \\
\hline Polygonum amphibium & Polygonaceae & Water smartweed \\
\hline
\end{tabular}


Table I-3. Spectes Observed between Allard Pumphouse and 100-K Area.

\begin{tabular}{|l|l|l|}
\hline \multicolumn{1}{|c|}{ Spectes } & \multicolumn{1}{c|}{ Family } & \multicolumn{1}{c|}{ Common name } \\
\hline Rumex salicifolius & Polygonaceae & Willow dock \\
\hline Montia perfoliata & Portulacaceae & Miner's lettuce \\
\hline Delphinium nuttallianum & Ranunculaceae & Upland larkspur \\
\hline Potentilla rivalis & Rosaceae & Brook cinquefoll \\
\hline Gallium aparine & Rubiaceae & Cleavers \\
\hline Salix exigua & Salicaceae & Coyote willow \\
\hline Verbascum thapsus & Scrophulariaceae & Wooly mullein \\
\hline Veronica anagallis-aguatica & Scrophulariaceae & water speedwell \\
\hline Ulmus pumila & Ulmaceae & Siberian Elm \\
\hline Verbena bracteata & Verbenaceae & Bracted verbena \\
\hline Verbena hastata & Verbenaceae & Blue verbena \\
\hline
\end{tabular}


Table 1-4. Species Observed along Shoreline between 100-K and 100-N Areas. (sheet 1 of 3 )

\begin{tabular}{|c|c|c|}
\hline Species & Family & Common name \\
\hline Lomatium grayi & Aplaceae & Gray's desert-parsley \\
\hline Apocynum cannabinum & Apocynaceae & Common dogbane \\
\hline Asclepias speciosa & Asclepladaceae & Showy milkweed. \\
\hline Achillea millefolium & Asteraceae & Yarrow \\
\hline Antennaria umbrinella & Asteraceae & Umber pussytoes \\
\hline Artemisia campestris & Asteraceae & Pactflc sage \\
\hline Artemisia lindleyana & Asteraceae & Columbia river mugwort \\
\hline Artemisia ludoviciana & Asteraceae & Prairle sage \\
\hline Aster hesperius & Asteraceae & Western marsh aster \\
\hline Centaurea diffusa & Asteraceae & Diffuse knapweed \\
\hline Cichorium intybus & Asteraceae & Chicory \\
\hline Cirsium vulgare & Asteraceae & Bull thistle \\
\hline Conyza canadensis & Asteraceae & Horseweed \\
\hline Coreopsis atkinsoniana & Asteraceae & Tickseed \\
\hline Gnaphal ium palustre & Asteraceae & Lowl and cudweed \\
\hline Helenium autumnale & Asteraceae & Sneezelveed \\
\hline Lactuca serriola & Asteraceae & Prickly lettuce \\
\hline Solidago graminifolia & Asteraceae & Bushy goldenrod \\
\hline Tragopogon dubius & Asteraceae & Salsify \\
\hline Xanthium strumarium & Asteraceae & Cocklebur \\
\hline Amsinckia Iycopsoides & Boraginaceae & Tarweed fiddleneck \\
\hline Myosotis micrantha & Boraginaceae & Blue forget-me-not \\
\hline Myosotis laxa & Boraginaceae & Small forget-me-not \\
\hline Cardamine pennsylvanica & Brassicaceae & Pennsylvania bittercress \\
\hline Draba verna & Brassicaceae & Spring whitlowgrass \\
\hline Rorippa columbiae & Brassicaceae & Columbia yellowcress \\
\hline Rorippa curvisiliqua & Brassicaceae & Western yellowcress \\
\hline Rorippa islandica & Brassicaceae & Marsh yellowcress \\
\hline Rorippa obtusa & Brassicaceae & Blunt leaf Yellowcress \\
\hline
\end{tabular}


Table 1-4. Spectes Observed along Shoreline between 100-K and 100-N Areas. (sheet 2 of 3 )

\begin{tabular}{|c|c|c|}
\hline Species & Family & Common name \\
\hline sisymbrium altissimum & Brassicaceae & Jim Hill Mustard \\
\hline Cerastium vulgatum & Caryophyllaceae & Common chickweed \\
\hline Dianthus armeria & Caryophyll aceae & Grass pink \\
\hline Holosteum umbellatum & Caryophyllaceae & Jagged chickweed \\
\hline Convolvulus arvensis & Convolvulaceae & Field bindweed \\
\hline Juniperus scopulorum & Cupressaceae & Rocky mt. juniper \\
\hline Carex aperta & Cyperaceae & Columbia sedge \\
\hline Carex athrostachya & Cyperaceae & Slenderbeak sedge \\
\hline Carex densa & Cyperaceae & Dense sedge \\
\hline Carex douglasit & Cyperaceae & Douglas' sedge \\
\hline Carex lenticularis & Cyperaceae & Kellogg sedge \\
\hline Eleocharis palustris & Cyperaceae & Common spikerush \\
\hline Equisetum hyemale & Equisetaceae & Dutch horsetall \\
\hline Lotus purshiana & Fabaceae & Spanish clover \\
\hline Lupinus lepidus & Fabaceae & Prairie lupine \\
\hline Medicago lupulina & Fabaceae & Black medick \\
\hline Melilotus alba & Fabaceae & White sweetclover \\
\hline Trifolium repens & Fabaceae & White clover \\
\hline Erodium cicutarium & Geraniaceae & Crane's bill \\
\hline Hypericum perforatum & Hypericaceae & St. Johnswort \\
\hline Juncus balticus & Juncaceae & Baltic rush \\
\hline Juncus tenuis & Juncaceae & slender rush \\
\hline Iriglochin palustre & Juncaginaceae & Marsh arrowgrass \\
\hline Lycopus americanus & Lamiaceae & Cutleaf water-horehound \\
\hline Mentha arvensis & Lamiaceae & Field mint \\
\hline Allium schoenoprasum & Lfliaceae & Chives \\
\hline Asparagus officinalis & L11 1aceae & Asparagus \\
\hline Marsilea vestita & Marsileaceae & Clover fern \\
\hline Morus alba & Moraceae & Mulberry \\
\hline
\end{tabular}


Table 1-4. Spectes Observed along Shoreline between 100-K and 100-N Areas. (sheet 3 of 3 )

\begin{tabular}{|c|c|c|}
\hline Specles & Family & Common name \\
\hline Eptloblum watsonif & Onagraceae & Watson's w11lowherb \\
\hline Denothere strigosa & Onagraceae & Common eveningprimrose \\
\hline Plantago lanceolata & Plantaginaceae & English plantain \\
\hline Plantago major & Plantaginaceae & Common plantain \\
\hline Agropyron dasytachyum & Poaceae & Thickspike wheatgrass \\
\hline Agrostis scabra & Poaceae & Ticklegrass \\
\hline Bromus tectorum & Poaceae & Cheatgrass \\
\hline Pantcum caplllare & Poaceae & Common witchgrass \\
\hline Phalaris arundinacea & Poaceas & Reed canarygrass \\
\hline Poa bulbose & Poaceae & Bulbous bluegrass \\
\hline Poa pratensis & Poaceae & Kentucky bluegrass \\
\hline Poa sandberait & Poaceae & Sandberg's bluegrass \\
\hline Poa scabreila & Poaceae & Pine bluegrass \\
\hline Polygonum aviculare & Polygonaceae & doorweed \\
\hline Rumex salicifolius & Polygonaceae & Wt1low dock \\
\hline Montia perfoliata & Portulacaceae & Miner's lettuce \\
\hline Ranunculus flammula & Ranuncul aceae & Creeping buttercup \\
\hline Potentilla rivalis & Rosaceae & Brook cinquefoll \\
\hline Salix exigua & Sallcaceae & Coyote willow \\
\hline Gratiola neglecta & Scrophulartaceae & Amertcan hedge-hyssop \\
\hline Limosella acaulis & Scrophulariaceae & Southern mudwort \\
\hline Verbascum thapsus & Scrophularlaceae & Wooly mullein \\
\hline Veronica americana & Scrophulartaceae & Brookl ime \\
\hline Veronica anagallis-aquatica & Scrophul artaceae & Water speedwell \\
\hline Veronica peregrina & Scrophulartaceae & Purslane speedwell \\
\hline Uimus pumila & Ulmaceae & Siberian elm \\
\hline Verbena bracteata & Verbenaceae & Bracted verbena \\
\hline
\end{tabular}


Table 1-5. Spectes Observed in Dryland Area above Shoreline between 100-K and 100-N Areas.

\begin{tabular}{|c|c|c|}
\hline Spectes & Famt1y & Common name \\
\hline Achillee millefollum & Asteraceae & Yarrow \\
\hline Agoseris heterophylla & Asteraceas & Annual mountain dandelion \\
\hline Ambroste ecenthicaroe & Asteraceas & Bur ragweed \\
\hline Artemisie campestris & Asteraceae & Pactific sage \\
\hline Artemisia tridentate & Asteraceas & Big sagebrush \\
\hline Centaurea diffusa & Asteraceae & Diffuse knapweed \\
\hline Cirsium arvense & Asteraceae & Canade thistle \\
\hline Chrysothamnus nauseosus & Asteraceae & Gray rabbitbrush \\
\hline Chrysothamus viscidiflorus & Asteraceae & Green rabbttbrush \\
\hline Erigeron pumilus. & Asteraceae & Shaggy fleabane \\
\hline Machaeranthere canescens & Asteraceae & Hoary aster \\
\hline Solldago sp. & Asteraceae & Goldenrod \\
\hline Amsinckie Iycopsotdes & Boraginaceae & Tamweed fiddleneck \\
\hline Amsinckie tessellate & Boraginaceae & Tessellate fiddleneck \\
\hline Descurainia pinnate & Brassicaceae & Tansymustard \\
\hline Lepidium perfol iatum & Brassicaceae & Clasping pepperweed \\
\hline Lepidium virginicum & Brassicaceae & Tall pepperweed \\
\hline sisymbrium altissimum & Brassicaceae & Jim Hill Mustard \\
\hline Holosteum umbellatum & Caryophyllaceae & Jagged chlckweed \\
\hline Sphaeralcea munroana & Malvaceae & Globemallow \\
\hline Agropyron dasytachyum & Poaceae & Thicksplke wheatgrass \\
\hline Bromus tectorum & Poaceae & Cheatgrass \\
\hline Elymus cinereus & Poaceae & Wildrye \\
\hline Poa sandberait & Poaceas & Sandberg's bluegrass \\
\hline Sporobolus cryptandrus & Poaceae & Sand Dropseed \\
\hline
\end{tabular}


Table 1-6. Spectes Observed along Shorel ine between 100-N and 100-D Areas. (sheet 1 of 3 )

\begin{tabular}{|c|c|c|}
\hline Species & Family & Common nume \\
\hline Lometiun arayt & Aplacene & Gray's desertparsley \\
\hline Apocynum cennebinum & Apocynacene & Common Dogbane \\
\hline Ascleptes spectose & Asclepiedicene & Showy milkweed \\
\hline Achtllee atllefoliun & Asterecene & Yerrow \\
\hline Artemiste cenpestris & Astericene & Pectflc sage \\
\hline Artemiste Iindlevene & Asterncese & Columble river muswort \\
\hline Artentste ludovictene & Astericene & Pratrie sase \\
\hline Artealste tridentate. & Astericene & B10 Sagebrush \\
\hline Aster occidentelis & Asteracese & Hestern mountaln aster \\
\hline Centauree diffuse & Asternceue & Diffuse Knapweed \\
\hline Cirstun ervense & Astericene & Caned thistle \\
\hline Chrysotheanus neuseosus & Asteracese & Gray rabbitbrush \\
\hline Coreopsts atkinsonitene & Asteracese & Tickseed \\
\hline Galllendle eristate & Asteraceese & Blanket flower \\
\hline Soltduge ereatnifolie & Astericeese & Bushy goldenrod \\
\hline Tragopogon dublus & Asteraceae & Salsify \\
\hline Xanthium strumeriun & Astericene & Cocklebur \\
\hline Amsinckle Iycopsoides & Boraginaceae & Tarweed fiddleneck \\
\hline Amsinckie tessellate & Boraginaceae & Tessellate fiddleneck \\
\hline Myosotis lexa & Boraginaceae & Small forget-me-not \\
\hline Descurainie pinnate & Brasslcaceae & Tansymustard \\
\hline Drabe verna & Brasstcaceae & Spring whit lowgrass \\
\hline Lepidiun perfoliatum & Brasstcaceae & Clasping pepperweed \\
\hline Sisymbrium altissimum & Brasstcacene & Jim HAll mustard \\
\hline Cerastium vulgatum & Caryophyllacese & Common chlckweed \\
\hline Holosteum umbellatum & Caryophyllaceae & Jagged chickweed \\
\hline Grayia spinose & Chenopodiaceae & Spiny hopsage \\
\hline Convolvulus arvensis & Convolvulaceae & Field bindweed \\
\hline Carex densa & Cyperaceae & Dense sedge \\
\hline
\end{tabular}


Table 1-6. Spectes Observed along Shorel ine between 100-N and 100-0 Areas. (shent 2 of 3 )

\begin{tabular}{|c|c|c|}
\hline Spectes & Funtly & Common name \\
\hline Cerex lenuatnose & cyperecene & woolly sedge \\
\hline Cerex oreegreallis & cyperecees & Clustered field sedoe \\
\hline Equitsetun lenvientun & Equisetecene & Smooth scouringrush \\
\hline Lupinus wyethil & Fabrecere & Eyeth's lupine \\
\hline Medicare lupuline & Febecene & Black madick \\
\hline Mediciee setive & Febrecene & Alfulfe \\
\hline Alliun rebinsenti & Lillecere & Robinson onton \\
\hline Alliun schoenopresun & Hilacene & Chives \\
\hline Asperequs officinile & L111ecene & Asparaqus \\
\hline Spheerelae aunroene & Molvecene & Globe mullow \\
\hline Morus albe & Moracene & Mulberry \\
\hline Plentege lenceelate & Plenterinacere & English plentain \\
\hline Plentuge majer & Plantaginaceee & Common plentain \\
\hline Plentuge peturenta & Plenteginaceae & Indien wheat \\
\hline Agropyron desytechyun & Poncene & Th1ckspike whentgrass \\
\hline Agropyron spicatun & Poacene & Bluebunch wheatgress \\
\hline Agrostis scabre & Porcene & Tlcklegrass \\
\hline Bromus tectorun & Porcene & Cheatorass \\
\hline Festuce erundinecee & Poncene & Tall fescue \\
\hline Oryzopsis hymenotdes & Poncene & Indien ricegrass \\
\hline Phaleris erundinceee & Porcene & Reed canarygrass \\
\hline Poe bulbose & Porcene & Bulbous bluegrass \\
\hline Poe prutensis & Poaceae & Kentucky bluegrass \\
\hline Sporobolus cryptendrus & Poaceare & Sand dropseed \\
\hline Stipe comate & Porcene & Needle-and-thread \\
\hline Eriogonum niveun & Polygonaceae & Snow buckwheat \\
\hline Rumex selicifollus & Polygonacene & Willow dock \\
\hline Montia perfollata & Portulacaceae & Miner's lettuce \\
\hline Potentilla rivalis & Rosaceae & Brook cinquefoll \\
\hline
\end{tabular}


Table 1-6. Spectes Observed along Shoreline between 100-N and 100-D Areas. (thent 3 of 3 )

\begin{tabular}{|c|c|c|}
\hline Spectes & Fontly & Common name \\
\hline Rosen woodsll & Rosictene & Hoods's rose \\
\hline Veronice enequllis-equetian & serophuleriecene & Wuter speedivell \\
\hline Veronial peregrine & serophulertacene. & Pursline speedwell \\
\hline Verbescun theosus & Serophulartacene & Yoolly mullein \\
\hline Lyciun helintfoliun & Solenecree & Matrimony vine \\
\hline Uimus punilus & Uimacese & sibertan elm \\
\hline
\end{tabular}


Table 1-7. Spectes Observed at Gravel/Cobble Shelf below 100-D Area. (sheet 1 of 2)

\begin{tabular}{|c|c|c|}
\hline Spectes & Fantly & Common name \\
\hline Lomat lum grayl & Aplacene & Gray's desertparsley \\
\hline Ascleptas spectose & Ascleptadaceas & showy milkweed \\
\hline Achlllea millofoltum & Asteracese & yarrow \\
\hline Artemista campestris & Asteraceas & Pacific sage \\
\hline Artemista Iudovictana & Asteracese & pratrte sagebrush \\
\hline Centaurea diffusa & Asteracone & tumble knapweed \\
\hline Contaurea ropens & Asteracese & Russian knapweed \\
\hline Chrysothannus nauscosus & Astoracease & gray rabbttbrush \\
\hline Cirstum ervense & Asteraceace & Canada thistle \\
\hline Erigeron Inearis & Asteracene & dasert yellow dalsy \\
\hline Galllardia aristate & Astoracene & blanket flowar \\
\hline Lactuca serriola & Asteraceas & prlckly lettuce \\
\hline Taraxacum officinale & Astoraceas & dandelion \\
\hline Tragopogon dublus & Asteracene & yellow salsify \\
\hline sisymbrium altissinum & Brasstcaceae & Jim Hill's tumblemustard \\
\hline Salsola kall & Chenopodtaceas & Russian thistle \\
\hline Convolvulus arvensis & Convolvulaceas & fleld bindweed \\
\hline Elaeagnus angustifolla & El aeagnaceae & Russian dilve \\
\hline Lupinus Ieucophyllus & Fabaceae & velvet lupine \\
\hline Lupinus sericeus & Fabacean & stlky luptne \\
\hline Lupinus wyothil & Fabaceae & Wyath's lupine \\
\hline Medicago Iupulina & Fabaceace & black medtck \\
\hline Medicago sativa & Fabaceae & alfalfa \\
\hline Erodiun clcutariun & Gerantaceae & storksbill \\
\hline Ribes auraum & Grossulariaceae & golden currant \\
\hline Sphaeralcea munroana & Malvaceas & Munro's globemallow \\
\hline Morus alba & Moraceae & White mulberry \\
\hline Plantago lanceolata & Plantaginaceae & English plantaln \\
\hline Agropyron dasytachyum & Poaceae & thicksplke wheatgrass \\
\hline
\end{tabular}


WHC-EP-0620

Table 1-7. Specles Observed at Gravel/Cobble Shelf below 100-D Area. (sheet 2 of 2)

\begin{tabular}{|c|c|c|}
\hline Spectes & Family & Common name \\
\hline Agrostis albe & Poncene & redtop bentgrass \\
\hline Bromus tectorum & Poacene & cheatgrass \\
\hline Festuca arundinacea & Poaceare & tall fescue \\
\hline Oryzopsis hymenoldes & Poscene & Indian ricegrass \\
\hline Phalaris arundinacea & Poscene & reed canarygrass \\
\hline Pos pratensis & Poacene & Kentucky bluegrass \\
\hline Poe sandbergit & Poacose & Sandberg's bluegrass \\
\hline sitanion hystrix & Poscene & bott1ebrush grass \\
\hline Sporobolus cryptandrus & Polcene & sand dropseed \\
\hline Montie perfollate & Portulacaceae & miner's lottuce \\
\hline Verbascun thapsus & Scrophulartaceae & common mullein \\
\hline U1mus puatla & Vimacese & Stbertan elm \\
\hline
\end{tabular}


Table I-8. Species Observed in Sandy Area East of 100-D Area.

\begin{tabular}{|l|l|l|}
\hline \multicolumn{1}{|c|}{ Species } & \multicolumn{1}{|c|}{ Family } & \multicolumn{1}{c|}{ Common name } \\
\hline Cymopteris terebinthinus & Apiaceae & turpentine springparsley \\
\hline Achillea millefolium & Asteraceae & yarrow \\
\hline Artemisia tridentata & Asteraceae & big sagebrush \\
\hline Chrysothamnus nauseosus & Asteraceae & gray rabbitbrush \\
\hline Chrysothamnus viscidiflorus & Asteraceae & green rabbitbrush \\
\hline Cryptantha circumscissa & Boraginaceae & matted cryptantha \\
\hline Cryptantha fendleri & Boraginaceae & Fendler's cryptantha \\
\hline Cryptantha leucophaea & Boraginaceae & gray cryptantha \\
\hline Cryptantha pterocarya & Boraginaceae & winged cryptantha \\
\hline Erysimum asperum & Brassicaceae & rough wallflower \\
\hline Opuntia fragilis & Cactaceae & brittle pricklypear \\
\hline Astragalus caricinus & Fabaceae & buckwheat milkvetch \\
\hline Astragalus sclerocarpus & Fabaceae & stalked-pod milkvetch \\
\hline Psoralea lanceolata & Fabaceae & dune scurfpea \\
\hline Phacelia hastata & Hydrophyllaceae & whiteleaf scorplonweed \\
\hline Phacelia linearis & Hydrophyllaceae & threadleaf scorpionweed \\
\hline Sphaeralcea munroana & Malvaceae & Munro's globemallow \\
\hline Bromus tectorum & Poaceae & cheatgrass \\
\hline Oryzopsis hymenoides & Poaceae & indian ricegrass \\
\hline Sitanion hystrix & Poaceae & bottlebrush grass \\
\hline Stipa comata & Poaceae & needle-and-thread grass \\
\hline Phlox longifolia & Polemoniaceae & longleaf phlox \\
\hline & & \\
\hline
\end{tabular}


Table 1-9. Species Observed Downstream from 100-D Area, Boat Launch to River Mile 375. (sheet 1 of 2)

\begin{tabular}{|c|c|c|}
\hline Species & Family & Common name \\
\hline Lomatium grayi & Aplaceae & Gray's desertparsley \\
\hline Lomatium macrocarpum & Aplaceae & large-fruited lomatium \\
\hline Asclepias speciosa & Asclepiadaceae & showy milkweed \\
\hline Achillea millefolium & Asteraceae & yarrow \\
\hline Artemisia campestris & Asteraceae & Pacific sage \\
\hline Artemisia tridentata & Asteraceae & big sagebrush \\
\hline Centaurea diffusa & Asteraceae & diffuse knapweed \\
\hline Chrysothamnus nauseosus & Asteraceae & gray rabbitbrush \\
\hline Cirsium arvense & Asteraceae & Canada thistle \\
\hline Coreopsis atkinsoniana & Asteraceae & Columbia tickseed \\
\hline Erigeron pumilus & Asteraceae & shaggy fleabane \\
\hline Tragopogon dubius & Asteraceae & yellow salsify \\
\hline Xanthium strumarium & Asteraceae & cocklebur \\
\hline Amsinckia lycopsoides & Boraginaceae & fiddleneck \\
\hline Myosotis micrantha & Boraginaceae & blue forget-me-not \\
\hline Descurainia pinnata & Brassicaceae & western tansymustard \\
\hline Draba verna & Brassicaceae & spring whitlowgrass \\
\hline Lepidium perfoliatum & Brassicaceae & clasping pepperweed \\
\hline Lepidium virginicum & Brassicaceae & tall pepperweed \\
\hline Lesquerella douglasit & Brassicaceae & Columbia bladderpod \\
\hline Rorippa curvisiliqua & Brassicaceae & western yellowcress \\
\hline sisymbrium altissimum & Brassicaceae & Jim Hill's tumblemustard \\
\hline Cerastium nutans & Caryophyllaceae & nodding chickweed \\
\hline Holosteum umbellatum & Caryophyllaceae & jagged chickweed \\
\hline Chenopodium leptophyllum & Chenopodiaceae & slimleaf goosefoot \\
\hline Salsola kali & Chenopodiaceae & Russian thistle \\
\hline Carex aperta & Cyperaceae & Columbia sedge \\
\hline Carex douglasii & Cyperaceae & Douglas' sedge \\
\hline Carex spp. & Cyperaceae & sedge species \\
\hline
\end{tabular}


Table I-9. Species Observed Downstream from 100-D Area, Boat Launch to River Mile 375. (sheet 2 of 2)

\begin{tabular}{|c|c|c|}
\hline Species & Family & Common name \\
\hline Eleocharis palustris & Cyperaceae & commun spikerush \\
\hline Equisetum arvense & Equisetaceae & common horsetail \\
\hline Psoralea lanceolata & Fabaceae & dune scurfpea \\
\hline Lupinus lepidus & Fabaceae & prairie lupine \\
\hline Lupinus wyethii & Fabaceae & Wyeth's Iupine \\
\hline Erodium cicutarium & Geraniaceae & storksbill \\
\hline Juncus balticus & Juncaceae & baltic rush \\
\hline Asparagus officinalis & Lillaceae & asparagus \\
\hline Sphaeralcea munroana & Malvaceae & globemallow \\
\hline Marsilea vestita & Marsileaceae & clover fern \\
\hline Agropyron caninum & Poaceae & slender wheatgrass \\
\hline Agropyron dasytachyum & Poaceae & thickspike wheatgrass \\
\hline Agropyron spicatum & Poaceae & bluebunch wheatgrass \\
\hline Agrostis alba & Poaceae & redtop bentgrass \\
\hline Bromus tectorum & Poaceae & cheatgrass \\
\hline Elymus cinereus & Poaceae & glant wildrye \\
\hline Oryzopsis hymenoides & Poaceae & indian ricegrass \\
\hline Phalaris arundinacea & Poaceae & reed canarygrass \\
\hline Poa bulbosa & Poaceae & bulbous bluegrass \\
\hline Poa nevadensis & Poaceae & Nevada bluegrass \\
\hline Poa pratensis & Poaceae & Kentucky bluegrass \\
\hline Poa sandbergit & Poaceae & Sandberg's bluegrass \\
\hline Sporobolus cryptandrus & Poaceae & sand dropseed \\
\hline Microsteris gracilis & Polemoniaceae & pink gracilis \\
\hline Potentilla rivalis & Rosaceae & brook cinquefoll \\
\hline Rosa woodsii & Rosaceae & Wood's rose \\
\hline Verbascum thapsus & Scrophulariaceae & common mullein \\
\hline Collinsia parviflora & Scrophulariaceae & small blue-eyed Mary \\
\hline
\end{tabular}


Table 1-10. Species Observed - River Mile 375 to 100-H Area. (sheet 1 of 3 )

\begin{tabular}{|c|c|c|}
\hline Species & Family & Common name \\
\hline Lomatium gray.i & Apiaceae & Gray's desertparsley \\
\hline Achillea millefolium & Asteraceae & yarrow \\
\hline Artemisia campestris & Asteraceae & Pacific sage \\
\hline Artemisia dracunculus & Asteraceae & tarragon \\
\hline Artemisia ludoviciana & Asteraceae & prairie sage \\
\hline Artemisia tridentata & Asteraceae & big sagebrush \\
\hline Centaurea diffusa & Asteraceae & tumble knapweed \\
\hline Centaurea repens & Asteraceae & Russian knapweed \\
\hline Chaenactis douglasif & Asteraceae & hoary falseyarrow \\
\hline Chrysothamnus nauseosus & Asteraceae & gray rabbitbrush \\
\hline Chrysothamnus viscidiflorus & Asteraceae & green rabbitbrush \\
\hline Cirsium arvense & Asteraceae & Canada thistle \\
\hline Erigeron filifolius & Asteraceae & threadleaf fleabane \\
\hline Erigeron poliospermus & Asteraceae & cushion fleabane \\
\hline Gaillardia aristata & Asteraceae & blanket flower \\
\hline Machaeranthera canescens & Asteraceae & hoary aster \\
\hline Solidago occidentalis & Asteraceae & western goldenrod \\
\hline Taraxacum officinale & Asteraceae & dandelion \\
\hline Tragopogon dubius & Asteraceae & yellow salsify \\
\hline Xanthium strumarium & Asteraceae & cocklebur \\
\hline Descurainia pinnata & Brassicaceae & western tansymustard \\
\hline Amsinckia 7ycopsoides & Boraginaceae & tarweed fiddleneck \\
\hline Amsinckia tessellata & Boraginaceae & tessellate fiddleneck \\
\hline Myosotis micrantha & Boraginaceae & blue forget-me-not \\
\hline Draba verna & Brassicaceae & spring whitlowgrass \\
\hline Lepidium densiflorum & Brassicaceae & prairie pepperweed \\
\hline Lepidium perfoliatum & Brassicaceae & clasping pepperweed \\
\hline Lesquerella douglasif & Brassicaceae & Columbia bladderpod \\
\hline Rorippa columbiae & Brassicaceae & Columbia yellowcress \\
\hline Rorippa curvisiliqua & Brassicaceae & western yellowcress \\
\hline
\end{tabular}


Table I-10. Spectes Observed - River Mile 375 to 100-H Area. (sheet 2 of 3 )

\begin{tabular}{|c|c|c|}
\hline Species & Family & Common name \\
\hline Rorippa obtusa & Brassicaceae & bluntleaf yellowcress \\
\hline sisymbrium altissimum & Brasstcaceae & Jim Hill's tumblemustard \\
\hline Cleome lutea & Capparidaceae & yellow bee-plant \\
\hline Holosteum umbellatum & Caryophy11aceae & Jagged chickweed \\
\hline Sagina procumbens & Caryophyllaceae & procumbent pearlwort \\
\hline Chenopodium album & Chenopodiaceae & lamb's quarters \\
\hline Chenopodium leptophyllum & Chenopod laceae & slimleaf goosefoot \\
\hline Grayia spinosa & Chenopodiaceae & spiny hopsage \\
\hline Salsola kali & Chenopod laceae & Russian thistle \\
\hline Juniperus scopulorum & Cupressaceae & Rocky Mountain juniper \\
\hline Carex lanuginosa & Cyperaceae & wooly sedge \\
\hline Carex douglasif & Cyperaceae & Douglas sedge \\
\hline Cyperus aristatus & Cyperaceae & awned flatsedge \\
\hline Equi setum arvense & Equisetaceae & common horsetall \\
\hline Equisetum spp. & Equisetaceae & horsetail \\
\hline Lupinus lepidus & Fabaceae & prairle lupine \\
\hline Lupinus sericeus & Fabaceae & silky lupine \\
\hline Lupinus wyethit & Fabaceae & Wyeth's lupine \\
\hline Medicago lupulina & Fabaceae & black medick \\
\hline Robinia psuedo-acacia & Fabaceae & black locust \\
\hline Erodiun cicuiarium & Gerantaceae & storksbill \\
\hline Ribes aureum & Grossulariaceae & golden current \\
\hline Hypericum perforatum & Hypericaceae & St. Johnswort \\
\hline Juncus tenuis & Juncaceae & slender rush \\
\hline Allium cernuum & Liliaceae & nodding onion \\
\hline Allium robinsonif & Liliaceae & Robinson's onion \\
\hline Asparagus officinalis & Liliaceae & asparagus \\
\hline Sphaeralcea munroana & Malvaceae & Munro's globemallow \\
\hline Marsilea vestita & Mars lleaceae & clover fern \\
\hline Morus alba & Moraceae & Mulberry \\
\hline
\end{tabular}


WHC-EP-0620

Table I-10. Spectes Observed - River Mile 375 to 100-H Area. (sheet 3 of 3 )

\begin{tabular}{|l|l|l|}
\hline \multicolumn{1}{|c|}{ Spectes } & \multicolumn{1}{|c|}{ Family } & \multicolumn{1}{c|}{ Common name } \\
\hline Plantago patagonica & Plantaginaceae & indian wheat \\
\hline Agropyron dasytachyum & Poaceae & thickspike wheatgrass \\
\hline Agropyron spicatum & Poaceae & bluebunch wheatgrass \\
\hline Agrostis alba & Poaceae & redtop bentgrass \\
\hline Aristida longiseta & Poaceae & red three-awn \\
\hline Bromus tectorum & Poaceae & cheatgrass \\
\hline Elymus cinereus & Poaceae & glant wildrye \\
\hline Hordeum glaucum & Poaceae & seagreen barley \\
\hline Koeleria cristata & Poaceae & prairle Junegrass \\
\hline Poa pratensis & Poaceae & Kentucky bluegrass \\
\hline Poa sandbergil & Poaceae & Sandberg's bluegrass \\
\hline Poa nevadensis & Poaceae & Nevada bluegrass \\
\hline Phalaris arundinacea & Poaceae & reed canarygrass \\
\hline Sporobolus cryptandrus & Poaceae & sand dropseed \\
\hline Stipa comata & Poaceae & needle-and-thread \\
\hline Collomia linearis & Polemoniaceae & narrowleaf collomta \\
\hline Gilia mirlutiflora & Polemoniaceae & smallflower gilia \\
\hline Microsteris gracilis & Polemoniaceae & pink microsteris \\
\hline Polemonium micranthum & Polemoniaceae & annual Jacob's ladder \\
\hline Eriogonum compositum & Polygonaceae & northern buckwheat \\
\hline Polygonum convolvulus & Polygonaceae & climbing bindweed \\
\hline Rumex crispus & Polygonaceae & curly dock \\
\hline Ranunculus testiculatus & Ranunculaceae & bur buttercup \\
\hline Potentilla rivalis & Rosaceae & brook cinquefoll \\
\hline Prunus armeniaca & Rosaceae & apricot \\
\hline Rosa woodsii & Rosaceae & Wood's rose \\
\hline Salix spp. & Salicaceae & willow \\
\hline Collinsia parviflora & Scrophulariaceae & small blue-eyed Mary \\
\hline Verbascum thapsus & Scrophulariaceae & common mullein \\
\hline Veronica anagallis-aquatica & Scrophulariaceae & water speedwell \\
\hline Verbena bracteata & Verbenaceae & bracted verbena \\
\hline
\end{tabular}


Table 1-11. Species Observed near 100-H Reactor. (sheet 1 of 2)

\begin{tabular}{|c|c|c|}
\hline Species & Family & Common name \\
\hline Cymopteris terebinthinus & Aplaceae & turpentine springparsley \\
\hline Lomatium grayi & Aplaceae & Gray's desertparsley \\
\hline Achillea millefolium & Asteraceae & yarrow \\
\hline Artemisia campestris & Asteraceae & Pactfic sage \\
\hline Artemisia tridentata & Asteraceae & big sagebrush \\
\hline Chrysothamnus nauseosus & Asteraceae & gray rabbitbrush \\
\hline Cirsium arvense & Asteraceae & Canada thistle \\
\hline Coreopsis atkinsoniana & Asteraceae & Columbia tickseed \\
\hline Heterotheca villosa & Asteraceae & hatry golden-aster \\
\hline Lactuca serriola & Asteraceae & prickly lettuce \\
\hline Taraxacum officinale & Asteraceae & dandelion \\
\hline Tragopogon dubius & Asteraceae & yellow saisify \\
\hline Xanthium strumarium & Asteraceae & cocklebur \\
\hline Descurainia pinnata & Brassicaceae & western tansymustard \\
\hline Draba verna & Brasstcaceae & spring whitlowgrass \\
\hline Lepidium perfoliatum & Brassicaceae & clasping pepperweed \\
\hline Rorippa islandica & Brassicaceae & marsh yellowcress \\
\hline sisymbrium altissimum & Brassicaceae & Jim Hill's tumblemustard \\
\hline Dianthus armeria & Caryophyllaceae & grass pink \\
\hline Holosteum umbellatum & Caryophyll aceae & jagged chickweed \\
\hline Salsola kall & Chenopodiacece & Russian thistle \\
\hline Carex spp. & Cyperaceae & sedge \\
\hline Equisetum spp. & Equisetaceae & horsetail \\
\hline Melilotus alba & Fabaceae & white sweetclover \\
\hline Ribes aureum & Grossulariaceae & golden currant \\
\hline Juncus spp. & Juncaceae & baltic rush \\
\hline Morus alba & Moraceae & white mulberry \\
\hline Agropyron dasytachyum & Poaceae & thickspike wheatgrass \\
\hline Agrostis alba & Poaceae & redtop bentgrass \\
\hline
\end{tabular}


WHC-EP-0620

Table 1-11. Spectes Observed near 100-H Reactor. (sheet 2 of 2)

\begin{tabular}{|l|l|l|}
\hline \multicolumn{1}{|c|}{ Species } & \multicolumn{1}{c|}{ Family } & \multicolumn{1}{c|}{ Common name } \\
\hline Bromus tectorum & Poaceae & cheatgrass \\
\hline Phalaris arundinacea & Poaceae & reed canarygrass \\
\hline Sporobolus cryptandrus & Poaceae & sand dropseed \\
\hline Microsteris gracllis & Polemonlaceae & pink microsteris \\
\hline Montia perfoliata & Portulacaceae & miner's lettuce \\
\hline Rosa woodsil & Rosaceae & Wood's rose \\
\hline Salix spp. & Sallcaceae & Willow \\
\hline Verbascum thapsus & Scraphularlaceae & common mullein \\
\hline Collinsia parviflora & Scrophulariaceae & small blue-eyed Mary \\
\hline Mazus japonicus & Scrophularlaceae & Japanese mazus \\
\hline Lycium halimifolium & Solanaceae & matrimony vine \\
\hline
\end{tabular}

spp. = spectes, more than one. 
Table 1-12. Species Observed in the Vicinity of 100-F Area. (sheet 1 of 3)

\begin{tabular}{|c|c|c|}
\hline $\begin{array}{r}\text { Spectes } \\
\end{array}$ & Famtly & Common name \\
\hline Cymopteris terebinthinus & Aplaceae & Turpentine springparsley \\
\hline Lomatium grayi & Aplaceae & Gray's desertparsley \\
\hline Apocynum cannabinum & Apocynaceae & Common dogbane \\
\hline Asclepias spectosa & Asclepladaceae & Showy milkweed \\
\hline Achillea millefolium & Asteraceae & Yarrow \\
\hline Ambrosia acanthicarpa & Asteraceae & bursage \\
\hline Artemisia campestris & Asteraceae & Pactflc sage \\
\hline Artemista Iindleyana & Asteraceae & Columbia mugwort \\
\hline Artemisia ludoviclana & Asteraceae & prairte sage \\
\hline Artemisia tridentata & Asteraceae & big sagebrush \\
\hline Aster hesperius & Asteraceae & western marsh aster \\
\hline Centaurea diffusa & Asteraceae & diffuse knapweed \\
\hline Centaurea repens & Asteraceae & Russian knapweed \\
\hline Chrysothamnus nauseosus & Asteraceae & Gray rabbitbrush \\
\hline Coreopsis atkinsontana & Asteraceae & Columbia tickseed \\
\hline Gaillardia aristata & Asteraceae & blanketflower \\
\hline Machaeranthera canescens & Asteraceae & Hoary aster \\
\hline Solidago graminifolia & Asteraceae & bushy goldenrod \\
\hline Xanthium strumarium & Asteraceae & cocklebur \\
\hline Amsinckid Iycopsoides & Boraginaceae & Tarweed fiddleneck \\
\hline Amsinckia tessellata & Boraginaceae & Tessellate fiddleneck \\
\hline Myosotis micrantha & Boraginaceae & blue forget-me-not \\
\hline Descurainia pinnata & Brassicaceae & Tansymustard \\
\hline Draba verna & Brassicaceae & spring whitlowgrass \\
\hline Lepidium perfollatum & Brassicaceae & clasping pepperweed \\
\hline sisymbrium altissimum & Brassicaceae & Jim Hill mustard \\
\hline Holosteum umbell atum & Caryophyllaceae & Jagged chickweed \\
\hline Cerastium nutans & Caryophyllaceae & common chickweed \\
\hline Salsola kali & Chenopodiaceae & Russian thistle \\
\hline
\end{tabular}


Table 1-12. Spectes Observed in the Vicinity of 100-F Area. (sheet 2 of 3)

\begin{tabular}{|c|c|c|}
\hline Species & Fantly & Common name \\
\hline Convolvulus arvensis & Convolvulaceae & Field bindweed \\
\hline Juniperus occidentalis & Cupressaceae & western Juniper \\
\hline Carex densa & Cyperaceae & dense sedge \\
\hline Carex aperta & Cyperaceae & Columbla sedge \\
\hline Carex microptera & Cyperaceae & smallwinged sedge \\
\hline Cyperus erythrorhizos & Cyperaceae & red-awn flatsedge \\
\hline Eleocharis palustris & Cyperaceae & common spikerush \\
\hline Sctrpus amertcanus & Cyperaceae & threesquare bulrush \\
\hline Lupinus lepidus & Fabaceae & pratrle luptne \\
\hline Medicago Iupulina & Fabaceae & black medick \\
\hline Melliotus alba & Fabaceae & white sweetclover \\
\hline Robinila psuedo-acacta & Fabaceae & Black locust \\
\hline Swainsona salsula & Fabaceae & Salt rattlepod \\
\hline Erodiun cicutarium & Geraniaceae & Crane'sbill \\
\hline Hypericum perforatum & Hypericaceae & St. Johnswort \\
\hline Allium schoenoprasum & Lillaceae & chives \\
\hline Sphaeralcea munroana & Malvaceae & Globemallow \\
\hline Morus alba & Moraceae & Mulberry \\
\hline Plantago lanceolata & Plantaginaceae & English plantain \\
\hline Plantago patagonica & Plantag inaceae & Indian wheat \\
\hline Platanus occidentalis & Platanaceae & Sycamore \\
\hline Bromus tectorum & Poacede & cheatgrass \\
\hline Festuca arundinacea & Poacear & Tall fescue \\
\hline Sporobolus cryptandrus & Poaceae & Sand dropseed \\
\hline Phalaris arundinacea & Poaceas & reed canarygrass \\
\hline Poa bulbosa & Poaceae & bulbous bluegrass \\
\hline Poa sandbergit & Poaceae & Sandberg's Bluegrass \\
\hline Collomia Iinearis & Polemontaceae & narrowleaf collomta \\
\hline Eriogonum niveum & Polygonaceae & snow buckwheat \\
\hline
\end{tabular}


Table 1-12. Species Observed in the Vicinity of 100-F Area. (sheet 3 of 3)

\begin{tabular}{|l|l|l|}
\hline \multicolumn{1}{|c|}{ Specles } & \multicolumn{1}{c|}{ Famlly } & \multicolumn{1}{c|}{ Common name } \\
\hline Mont le perfollata & Portulacaceae & miner's lettuce \\
\hline Populus nigra & Salleaceae & Lombardy poplar \\
\hline Sallx exigua & Sallcaceae & Coyote wlllow \\
\hline Collinsia parviflora & Scrophulariaceae & small blue-eyed mary \\
\hline Verbascum thapsus & Scrophularlaceae & wooly mullein \\
\hline UImus punila & Ulmaceae & Siberlan elm \\
\hline Verbena bracteate & Verbenaceae & Bracted verbena \\
\hline
\end{tabular}


WHC-EP-0620

APPENDIX J

SELECTED METALS CONCENTRATIONS IN ALL MEDIA

J-1 
WHC-EP-0620

Th1s page intentionally left blank.

1

 
WHC-EP-0620
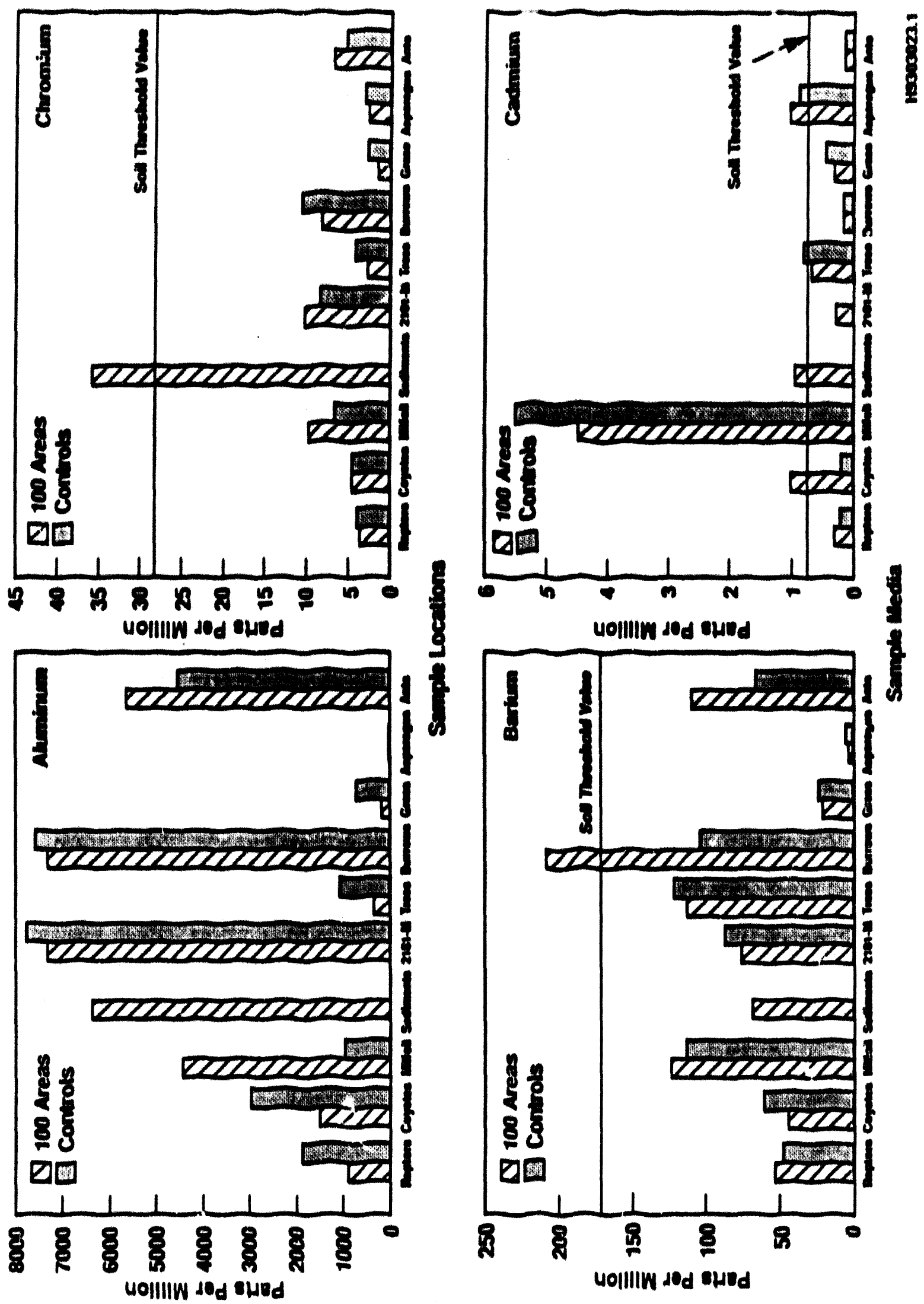
WHC-EP-0620
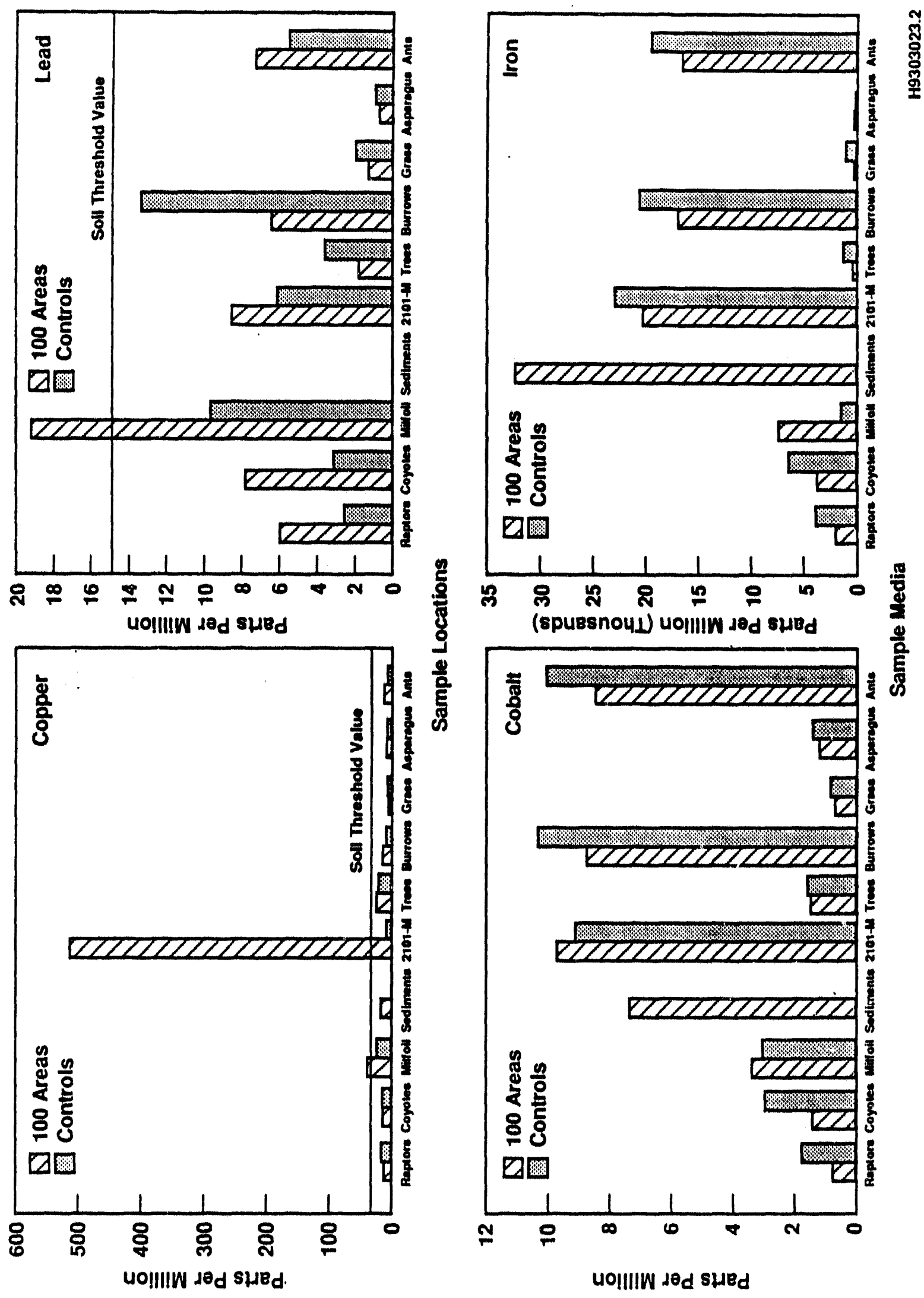
WHC-EP-0620
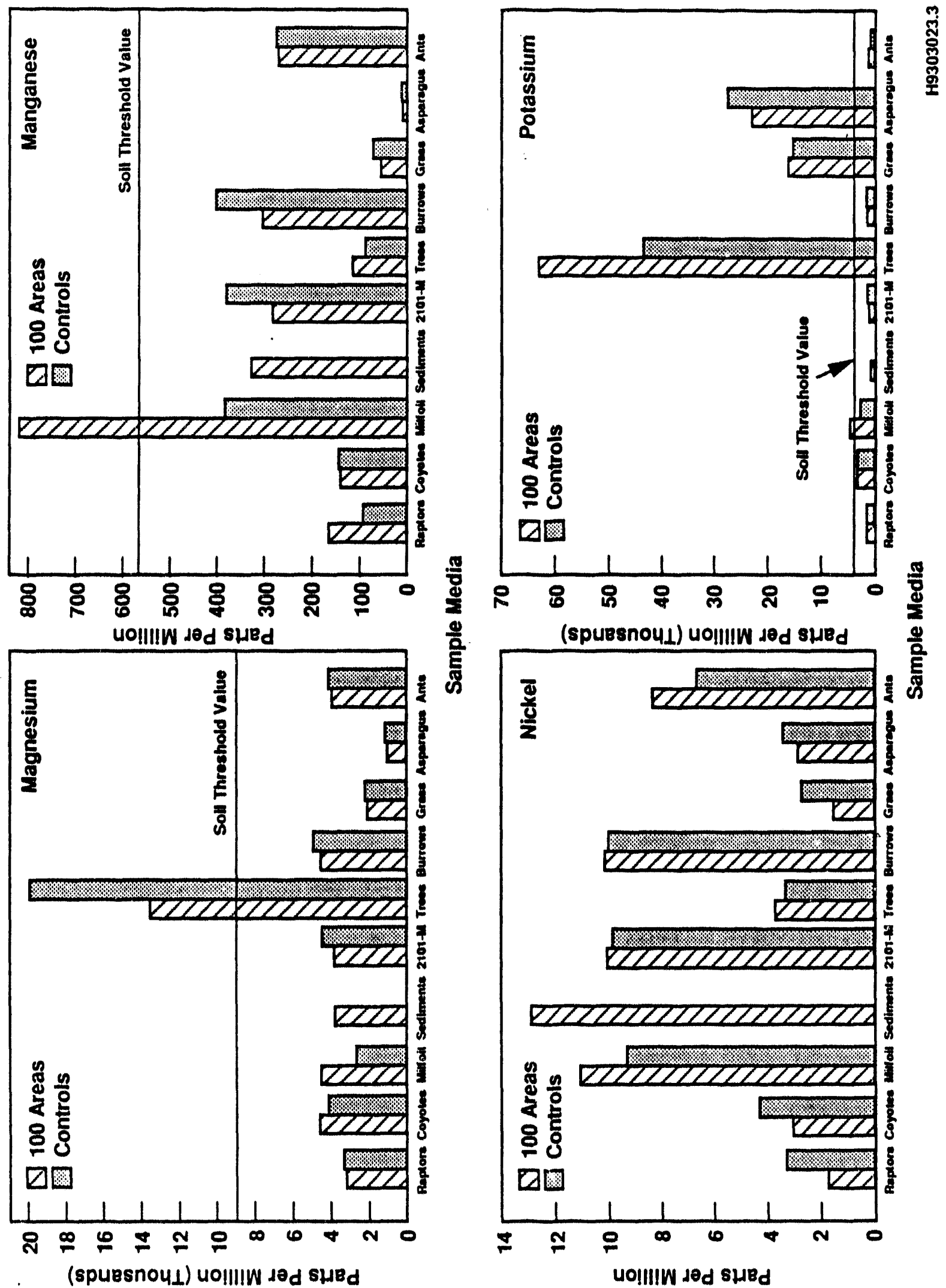
WHC-EP-0620
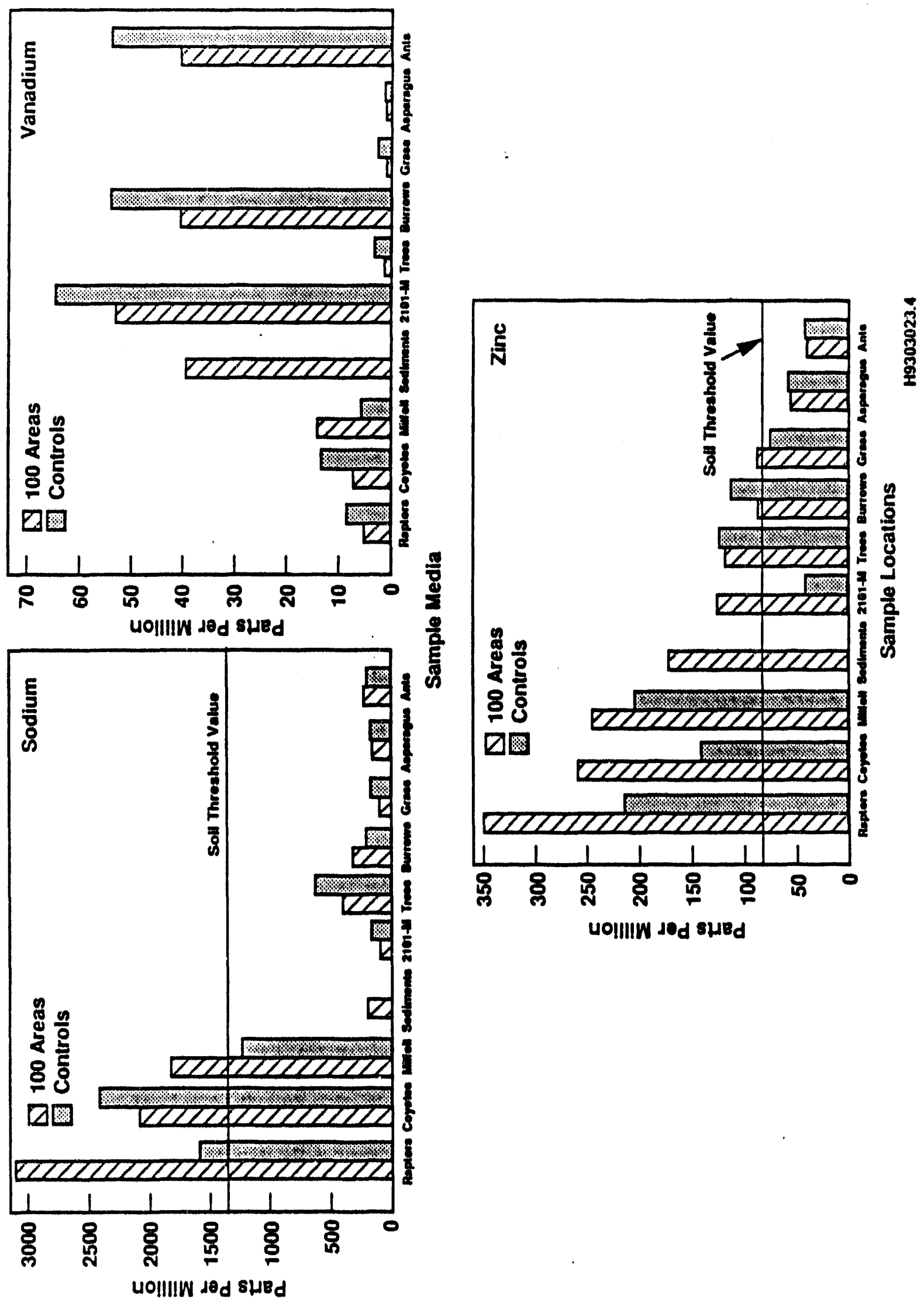
WHC-EP-0620

APPENDIX K

MARION OWNBEY HERBARIUM LETTERS 
WHC-EP-0620

This page intentionally left blank. 


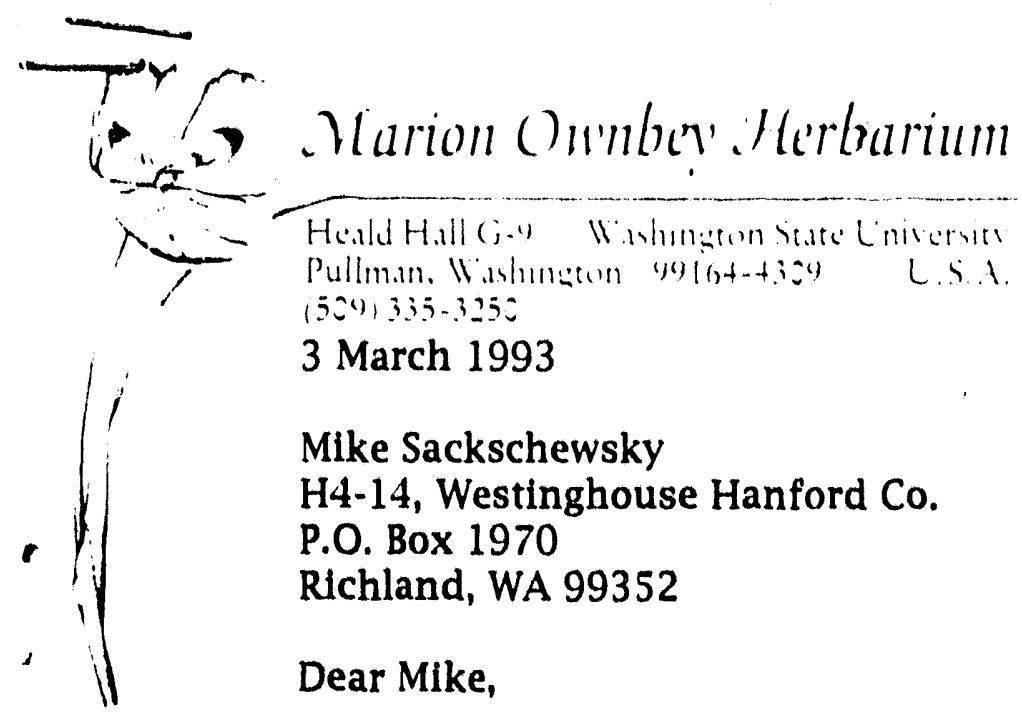

At last I have been able to look at the Carex specimens you sent for identification and confirmation. A list of my determinations follows. Two species are of particular interest: $C$. brevior and C. vulpinoidea. Your C. brevior is the first WS record from Benton County, and from looking at the Northwest Flora one would think that's west of the known distribution for that species. However, WS has specimens from Yakima and Kittitas Countles, so this is one of those cases where this herbarium wasn't consulted in gathering distribution information for the Flora.

With respect to $C$. vulpinoidea, your material really emphasizes the importance of having completely mature perigynia. Of course immaturity is often a problem with sedges, but in species such as $C$. vulpinoldea it is especially critical. This is because of the pithy (sometimes called "spongy") material which is within the perigynium wall near the base of the perigynium. This tissue is what gives the perigynium its characteristic shape and appearance, and it develops late in the maturation process. Consequently, even at a stage when perigynia of most sedge species are recognizable, those of $C$. vulpinoidea look more like $C$. dudleyi perigynia. Carex dudleyi is one of the taxa submerged in $C$. densa in the Northwest Flora. Carex dudleyi resembles $C$. vulpinoidea in size and general shape of perigynia, but if $C$. dudleyi develops pithy tissue (which it occasionally does), that tissue is distributed differently in the perigynium and gives the mature perigynium a shape quite different from that of mature $C$. vulpinoidea perigynla. The immature perigynla on your $C$. vulpinoidea collections look much like perigynla of $C$. dudleyt and could key to $C$. densa in the Northwest Flora. I I wonder if this could be the source of the older records for "C. densa."

If your collections hadn't been so complete, including inflorescences of different ages from the same plants, the situation would have been more difficult to resolve.

1 I should mention that there is a distinct difference in the thickness of the perigynium-$0.4-0.7 \mathrm{~mm}$ in $C$. vulpinoidea and $0.7-1.1 \mathrm{~mm}$ in $C$. dudleyl--but this character is not included in existing treatments (except of course 1993 Jepson!). 


$\begin{array}{ll}207 a & \text { C. lanuginosa Michaux } \\ 226 \mathrm{a} & \text { C. athrostachya Olney } \\ 227 \mathrm{a} & \text { C. lenticularis var. lipocarpa (Holm) L. Standley } \\ 240 \mathrm{a} & \text { !. C. lanuginosa } \\ 254 \mathrm{a} & \text { C. athrostachya } \\ 259 \mathrm{a} & \text { C. brevior (Dewey) Mackenzie } \\ 260 \mathrm{~b} & \text { C. vulpinoidea Michaux } \\ 278 \mathrm{a} & \text { C. vulpinoidea } \\ 279 \mathrm{a} & \text { C. athrostachya } \\ 290 \mathrm{a} & \text { C. vulpinoidea } \\ 304 \mathrm{a} & \text { C. vulpinoidea } \\ 309 \mathrm{a} & \text { C. vulpinoidea }\end{array}$

Did you receive the key excerpts I sent? By the way, if you're interested, the Jepson revision is out now.

I tried to phone you about these matters, and also because I've had an Inquiry on how much it costs to put together a manual for plants of a particular area, similar to your book on Hanford plants. What this person needs to know is roughly the total it might cost, not including the field work, but the time to organize, put everything together, printing costs, etc. Would you be able to help with this? It doesn't have to be precise at all, just a general range of what one might expect.

Thank you for sending Vascular Plants of the Hanford site; It really came out nicely. Thank you too for the sedge specimens; they will be a very nice addition to the herbarium,

Sincerely yours,

$$
i
$$

Joy Mastrogiuseppe 


\section{Washington \\ State University}

Department. Pullman, Washington 99164-4230, 509.335.3066

ir $4 x y$

\section{March 1993}

Mike Sackschewsky

H4-14, Westinghouse Hanford Co.

P.O. Box 1970

Richland, WA 99352

Dear Mike,

WS has only one Washington collection of Carex densa: Piper 6439, from Clark County, Vancouver, 2 June 1904. This is the record I was trying to remember. The Wahkiakum County specimen must be at UW. Evidently the species Just barely extends up into Washington, though other records might well turn up.

Enclosed is a copy of Steppe by Step.

Sincerely yours,<smiles></smiles>

Joy. Mastrogiuseppe 
WHC-EP-0620

DISTRIBUTION

Number of copies

OFFSITE

$3 \quad$ U.S. Environmental Protection Agency

Olympia, Washington

D. R. Einan

L. E. Gadbots

B5-01

P. S. Innis

B5-01

B5-01

1 U.S. Army Corps of Engineers Walla Walla, Washington

A. Foote

2 Washinaton Department of Ecology Mall Stop PV-11

Olympla, Washington 98504-8711

S. F. Cross

L. Goldstein

2 Washinaton Department of Ecology

7601 W. Clearwater

Sulte 102

Kennewick, Washington 99336

D. Teel

J. Phtilips

1 Washington Department of Wildlife

Lower River Road

Benton City, Washington 99320

L. Fitzner

1 Washington Department of Health Division of Radiation Protection $2 S$ 45th Avenue

Yakima, Washington 98908

R. A. Dantelson

1 Washinaton Department of Wild life 7601 W. Clearwater

Sulte 102

Kennewick, Washington 99336

J. $\mathrm{Ha} 11$ 
WHC-EP-0620

DISTRIBUTION (cont)

3

Golder Associates, Inc

Redmond, Washington 98052

Library

L. Swenson

2

II Corp

1045 Jadwin Ave

Sutte $C$

Richl and, Washington 99352

J. Chiramonte

D. A. Myers

ONSIIE

6

U.S. Department of EnerayRichland Field offtce

B. L. Foley

A5-19

E. D. Goller

R. D. Hildebrand

A5-19

R. G. McLeod

R. K. Stewart

K. M. Thompson

A5-55

A5-19

A5-19

A5-15

9 Paclfic Northwest Laboratory

E. J. Antonio

K6-61

C. J. Brandt

L. L. Cadwell

K6-06

K6-63

S. L. Friant

R. Mazalka

K6. 52

K6-60

T. M. Poston

K6-61

K. M. Probasco

K6-52

W. H. Rickard

K6-63

R. K. Woodruff

K6-61

38

Westinghouse Hanford Company

M. A. Adams

H6-01

S. W. Clark

H6-01

L. Dean

G6-75

T. W. Forns

H6-26

M. J. Galbralth

H6-02

K. A. Gano

$\times 0-21$

R. F. Giddings

N3-06

R. P. Henckel

H6-02 
WHC-EP-0620

\section{DISTRIBUTION (cont)}

L. C. Hulstrom

H6-03

A. R. Johnson

C. J. Kemp

C. D. Kramer

H6-30

H4-14

D. S. Landeen

N. K. Lane

K. M. Leonard

J. G. Lucas

R. M. Mitchell

H6-04

H4-14

H6-01

H6-22

H6- 04

K. L. Peterson

H6- 04

C. J. Perkins

H4-14

R. C. Roos

M. R. Sackschewsky

J. W. Schmidt

J. A. Stegen

R. S. Weeks

S. G. Wetss (10)

Central flies

Information Release Administration

$\times 0-21$

H6-04

H4- 14

H6-30

H6-02

H6-26

H6-02

18-04

H4-17 
WHC-EP-0620

This page intentionally left blank.

1 

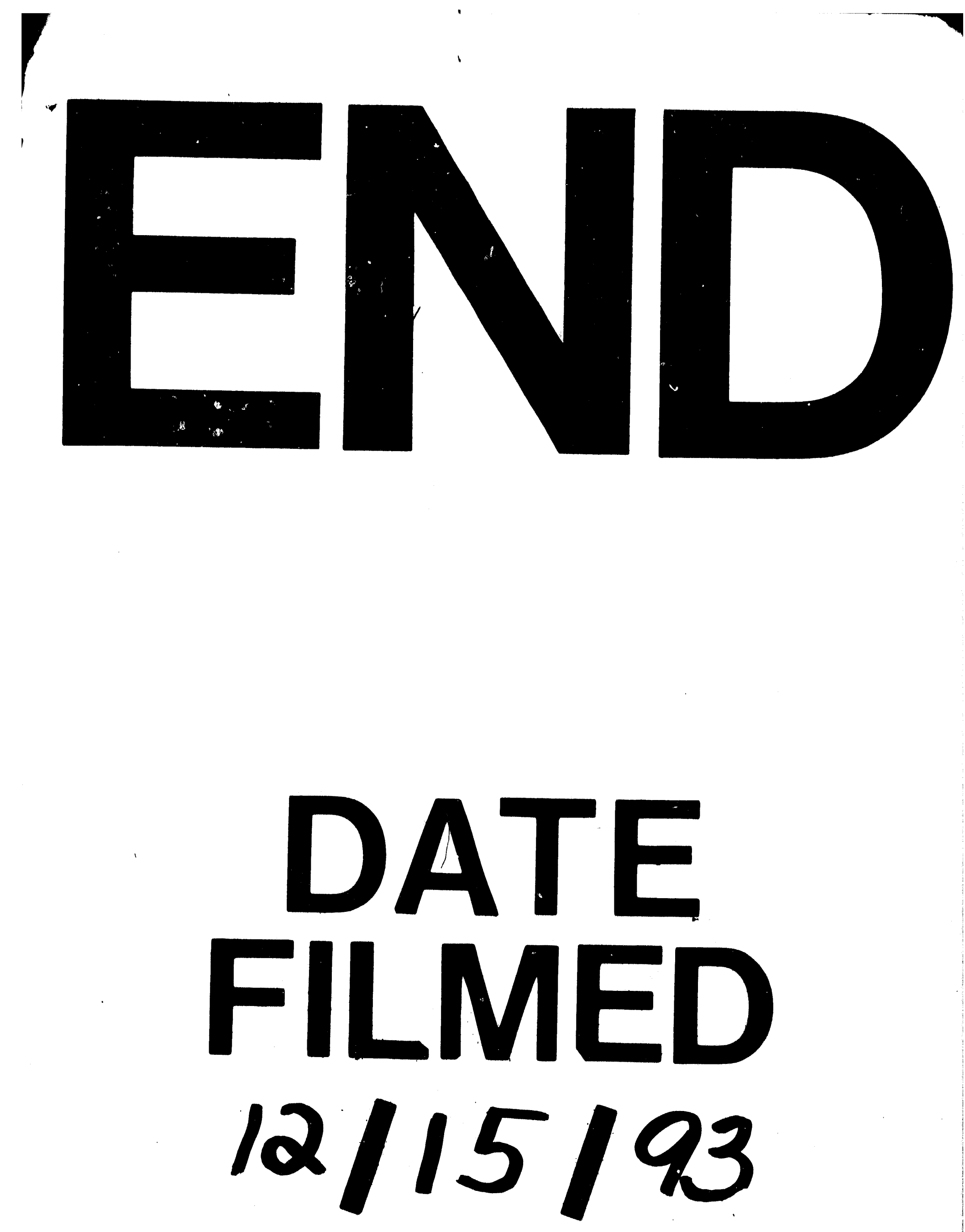
